\title{
Analysis on the mechanisms underlying the leupaxin-mediated progression of prostate cancer
}

\section{Doctoral thesis}

In partial fulfillment of the requirements for the degree

"Doctor rerum naturalium (Dr. rer. nat.)"

in the Molecular Medicine Study Program at the Georg-August University Göttingen

\author{
submitted by \\ Sascha Dierks \\ born in \\ Göttingen, Germany
}

Göttingen, 2014 
Members of the Thesis Committee:

Supervisor

Prof. Dr. rer.nat. Peter Burfeind

Department of Human Genetics, University Medical Center

Göttingen

Second member of the Thesis Committee

Prof. Dr. rer.nat. Dieter Kube

Department of Hematology and Oncology, University Medical Center Göttingen

Third member of the Thesis Committee

Prof. Dr. rer.nat. Holger Bastians

Department of Molecular Oncology, University Medical Center Göttingen

Date of Disputation: 


\section{AFFIDAVIT}

Herewith I declare that my doctoral thesis entitled: "Analysis on the mechanisms underlying the leupaxin-mediated progression of prostate cancer" has been written independently with no other sources and aids than quoted.

Göttingen, November 2014

Sascha Dierks 


\section{Table of Contents}

1 Introduction ...................................................................................1

1.1 The prostate carcinoma - at a glance …….........................................1

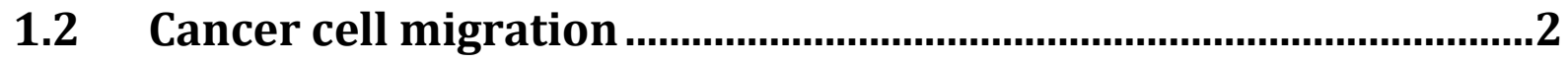

1.2.1 The essential mechanisms of cancer cell migration ..............................................2

1.2.2 Cytoskeletal rearrangement during cancer cell migration....................................

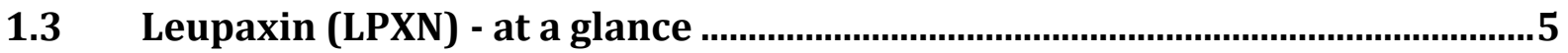

1.3.1 LPXN, a member of the paxillin protein family ...................................................

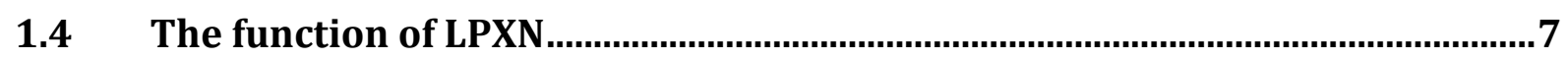

1.5 The function of LPXN in prostate cancer........................................................8

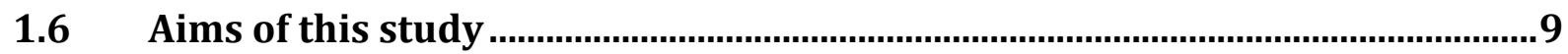

2 Materials and Methods ................................................................... 12

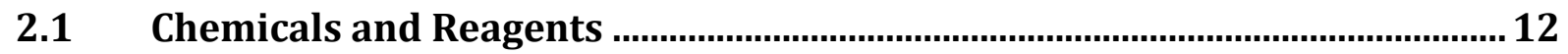

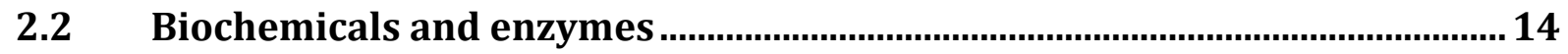

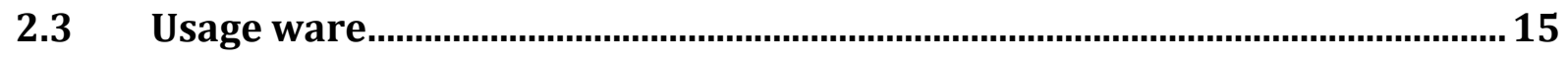

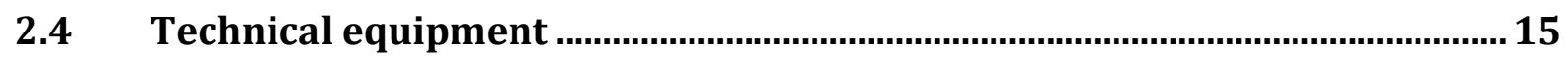

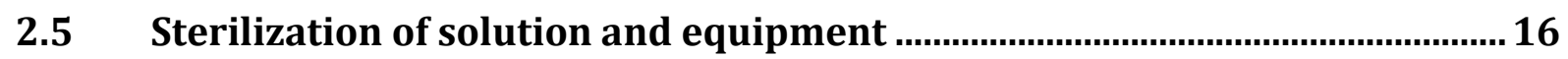

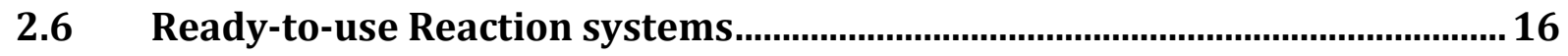

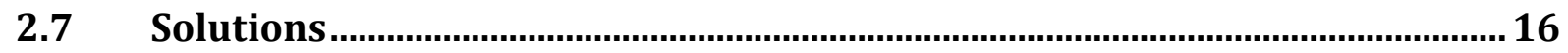

2.8 Culture media, antibiotics, agar plates.............................................................. 19

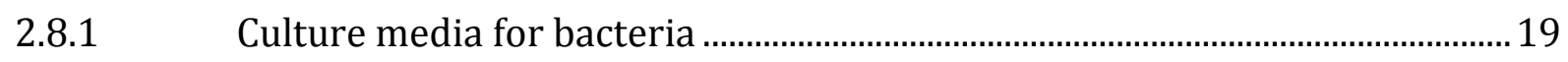

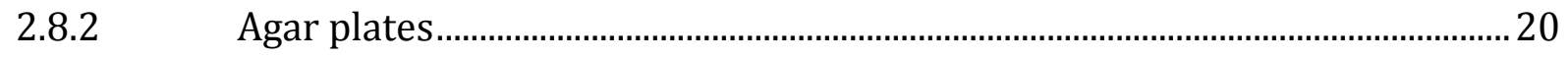

2.8.3 Culture media for eukaryotic cell cultures ........................................................... 20

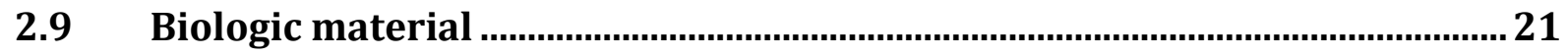

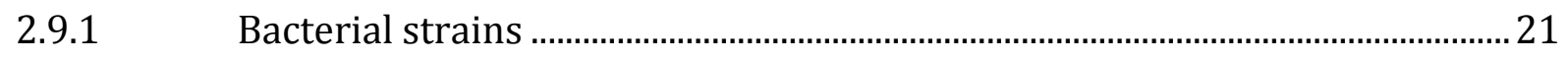

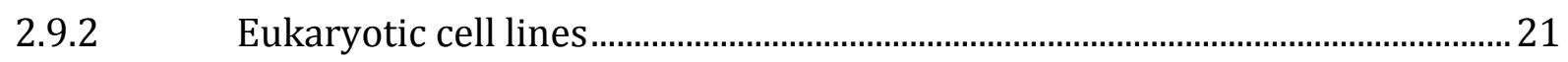

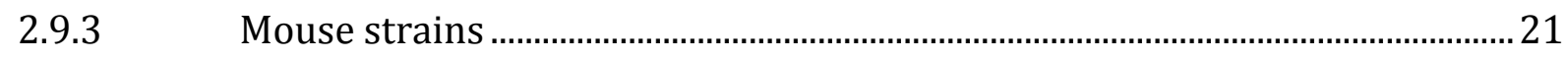

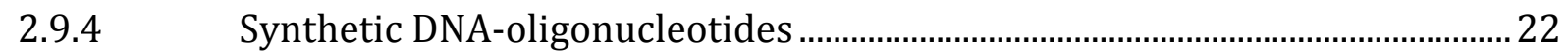

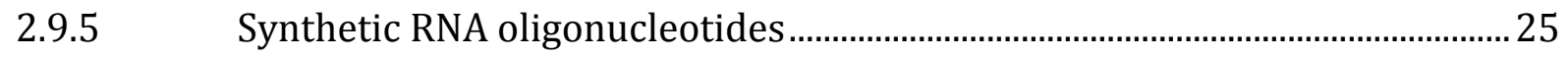

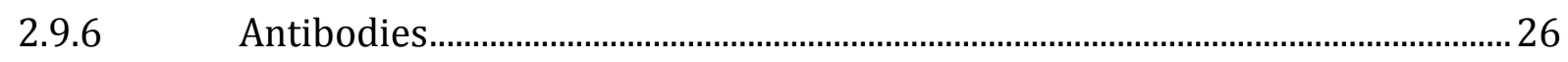

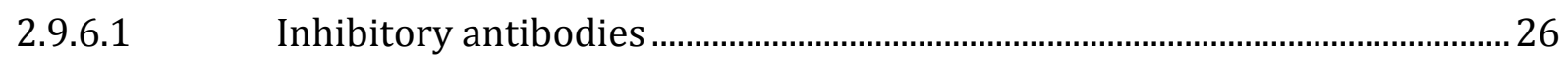

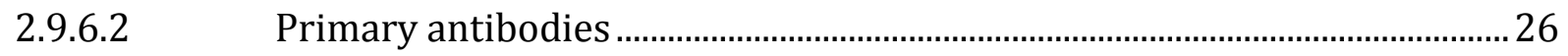




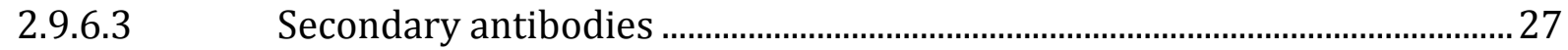

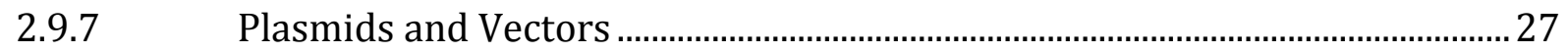

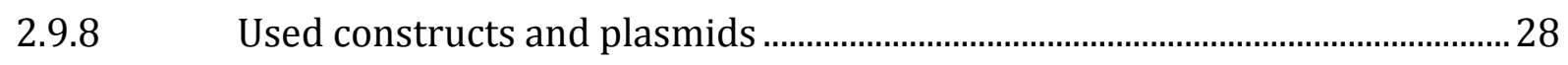

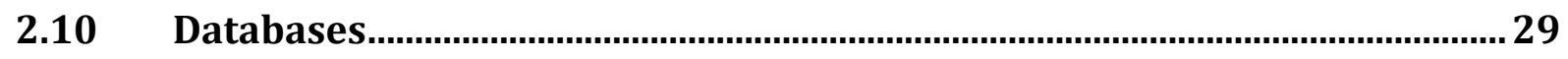

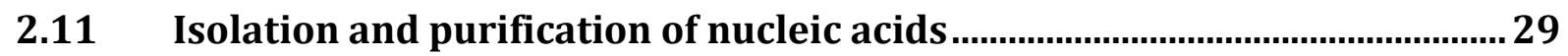

2.11.1 Minipreparation of plasmid DNA ………............................................................... 29

2.11.2 Establishment of bacterial glycerol stocks ........................................................ 30

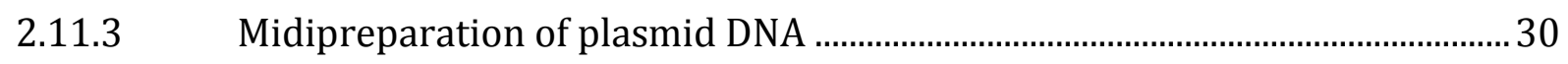

2.11.4 Isolation of total RNA from cell cultures............................................................ 30

2.11.5 Determination of nucleic acid concentration......................................................... 30

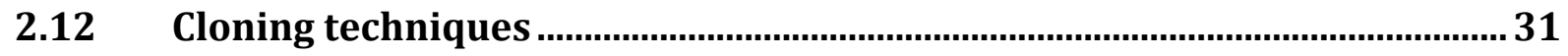

2.12.1 Cleavage of DNA with restriction endonucleases ............................................... 31

2.12.2 Isolation of DNA fragments from agarose gels...................................................... 31

2.12.3 Dephosphorylation of plasmid DNA ....................................................................... 31

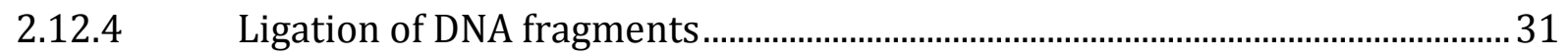

2.12.5 Subcloning of PCR and RT PCR products / TA cloning ……............................... 32

2.12.6 Transformation of competent bacteria with plasmid DNA ................................ 33

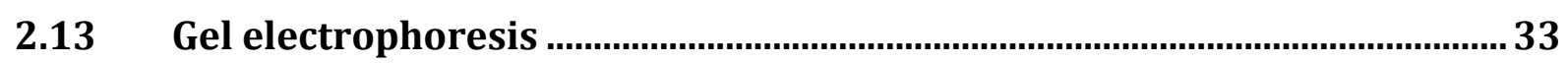

2.13.1 Agarose gel electrophoresis of DNA ……............................................................. 33

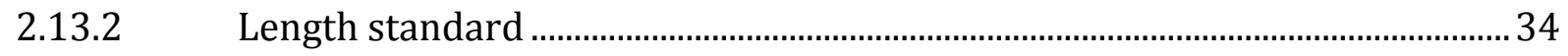

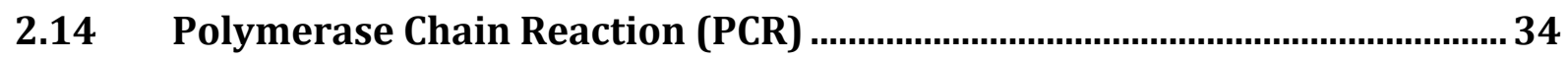

2.14.1 Amplification of DNA fragments from plasmid DNA …….................................. 34

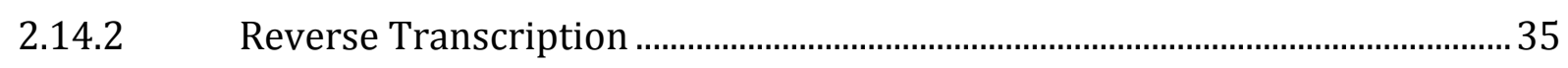

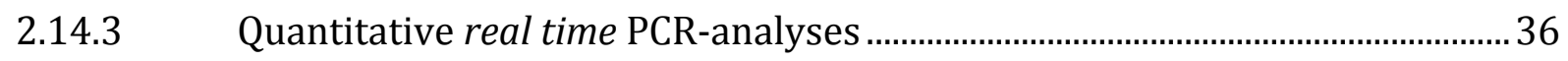

2.14.4 Sequence analysis / DNA-sequencing after Sanger.............................................. 38

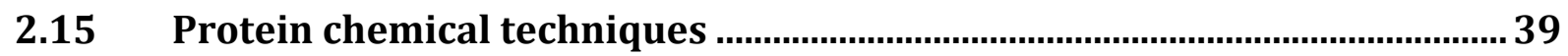

2.15.1 Isolation of total protein from cell cultures........................................................ 39

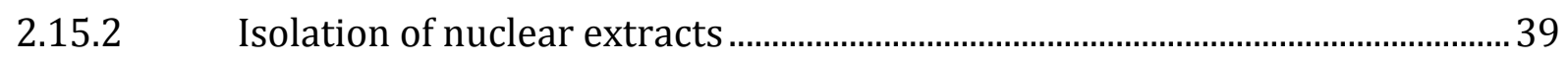

2.15.3 Determination of protein concentration ................................................................ 40

2.15.4 Sodium Dodecyl Sulfate Polyacrylamide Gel Electrophoresis

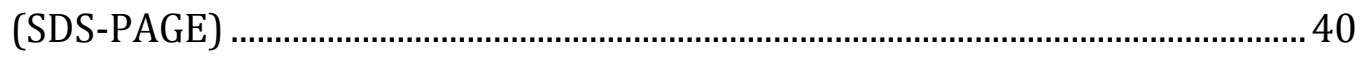

2.15.5 Transfer of proteins from Polyacrylamide gels to PVDF membranes............ 41

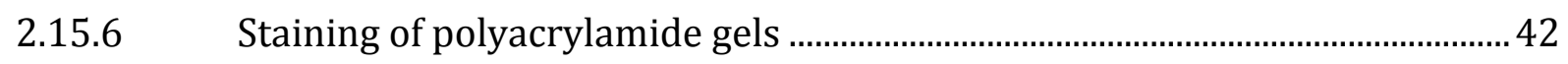

2.15.7 Incubation of protein-bound membranes with antibodies................................ 42 


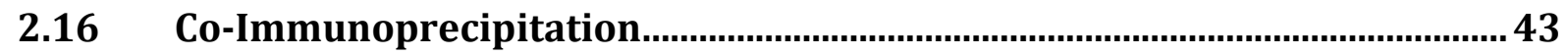

2.17 Duolink ${ }^{\circledR}$ II Proximity Ligation Assay (PLA).................................................44

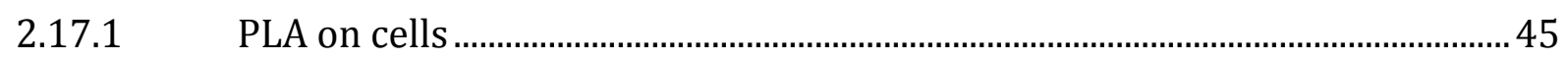

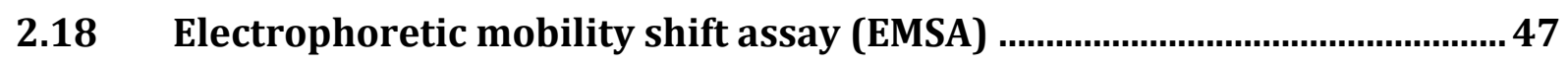

2.18.1 Casting of PAGE gels for EMSA …........................................................................ 47

2.18.2 Radioactive labeling using 5'-fill-in reaction ................................................... 47

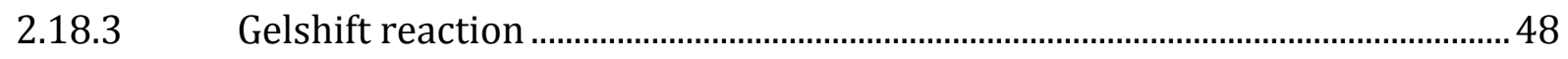

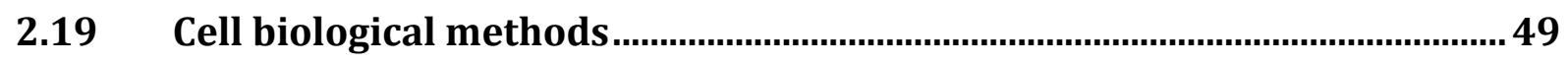

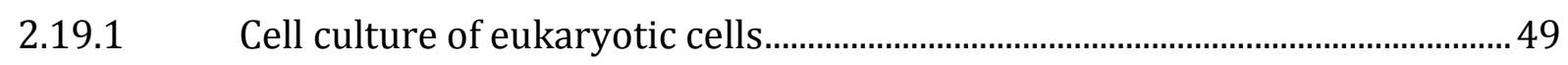

2.19.2 Cryo-preservation and revitalization of eukaryotic cells.................................... 49

2.19.3 Test for Mycoplasma contamination........................................................................ 50

2.19.4 Transfection of eukaryotic cells ................................................................................ 50

2.19.4.1 Transfection of plasmids into eukaryotic cells...............................................50

2.19.4.2 Transfection of small interfering RNA (siRNA) into eukaryotic cells...... 50

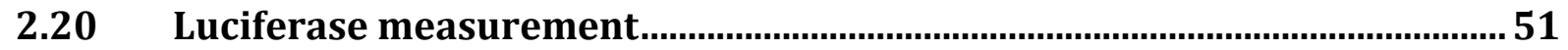

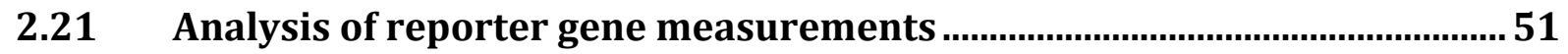

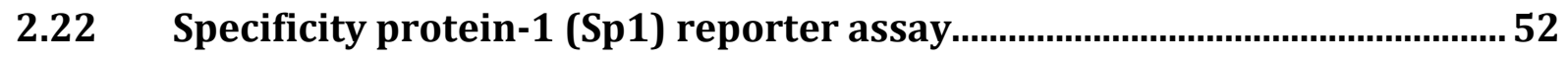

2.23 Functional analysis of eukaryotic cells …....................................................... 52

2.23.1 Colony formation assay ..................................................................................... 52

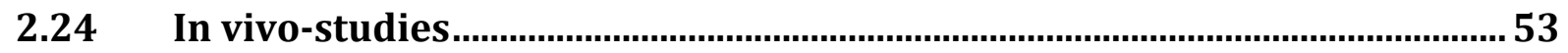

2.24.1 Generation of a conditional LPXN knockout mouse model ................................. 53

2.24.2 FLP recombination of LPXN knockout ES cells .................................................... 53

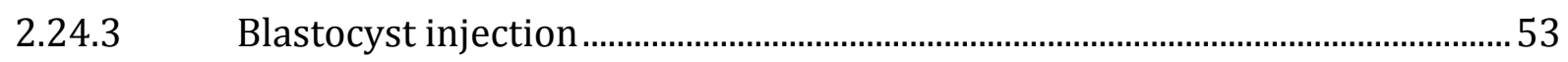

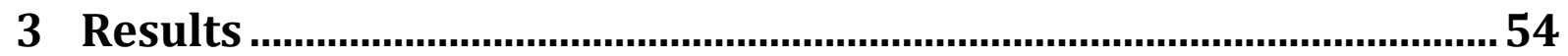

3.1 The interaction of LPXN with Caldesmon and it's relevance for PCa

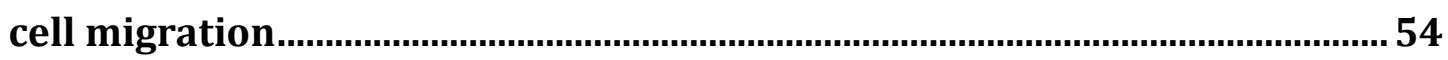

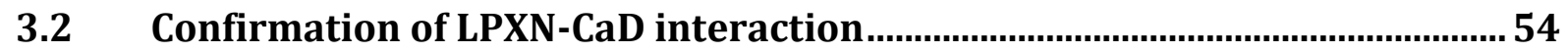

3.2.1 Co-immunoprecipitation of LPXN and l-CaD ……................................................ 54

3.2.2 In situ Proximity ligation assay (PLA) of LPXN and CaD ................................... 55

3.3 Increased interaction of LPXN and CaD during PCa cell migration ...............56

3.4 Increased interaction of LPXN and phosphorylated CaD during

PCa cell migration ......................................................................................... 58

3.5 Localization of pCaD-LPXN interaction during migration of PCa cells........ 59

3.6 LPXN interacts with the extracellular signal-regulated kinase (ERK) ......... 61 
3.7 Basal expression of ERK in PCa cell lines PC-3, DU145 and LNCaP............... 62

3.8 Increased interaction of LPXN and ERK during PCa cell migration ..............63

3.9 LPXN mediates phosphorylation status of CaD through interaction

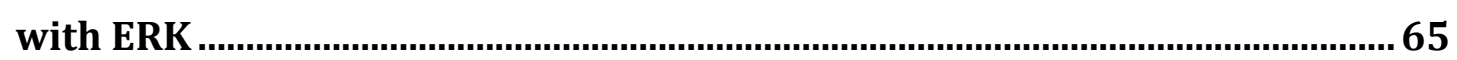

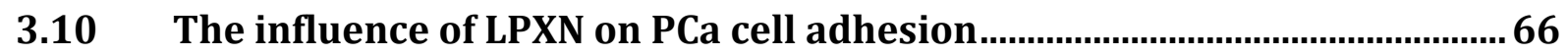

3.10.1 Expression of ITGB1 in the established PCa cell lines PC-3, DU145 and LNCaP

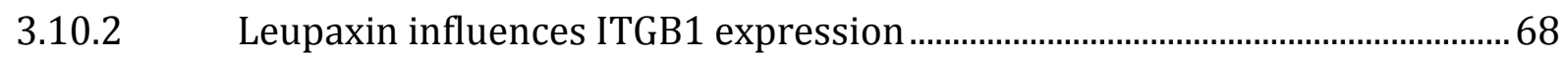

3.10.3 LPXN influences activity of the Specificity protein 1 (Sp1).............................. 72

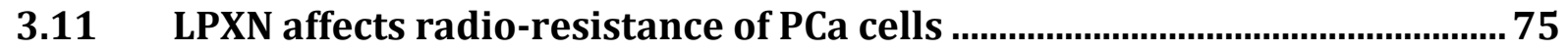

3.11.1 LPXN expression is enhanced upon ionizing radiation ..................................... 81

3.11.2 ITGB1 expression is enhanced upon ionizing radiation ...................................... 82

3.11.3 LPXN knockdown prevents radiation-induced upregulation of

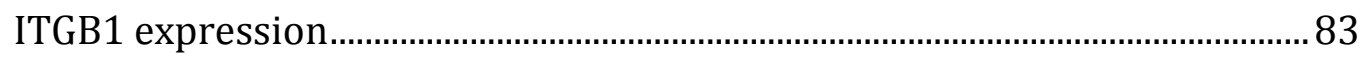

3.12 The interaction of LPXN and the adhesion molecule p120CTN ................... 85

3.12.1 The CTNND1 promoter is influenced by LPXN …………..................................... 87

3.12.2 LPXN does not directly bind to promoter sequences of the

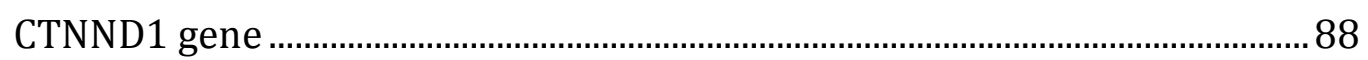

3.13 Generation of a LPXN gene trap conditional knockout mouse model........93

3.13.1 The LPXN targeted trap construct........................................................................... 93

3.13.2 Excision of reporter/selector cassette ................................................................. 94

3.13.3 First blastocyst injection of mouse embryonic stem cells .................................95

3.13.4 Cytogenetic analysis of mouse embryonic stem cells for

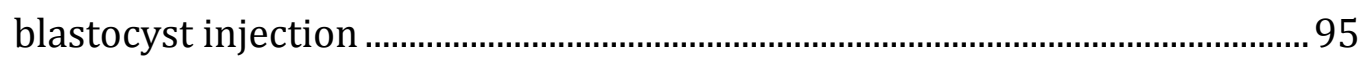

3.13.5 Rescue of differentiated mES cells by RESGRO ${ }^{\circledR}$ culture medium ....................96

3.13.6 Second blastocyst injection of mouse embryonic stem cells ............................ 96

3.13.7 Genotyping of LPXN19 chimera offspring......................................................... 97

3.13.8 Establishment of a homozygous LPXN flox/flox conditional knockout

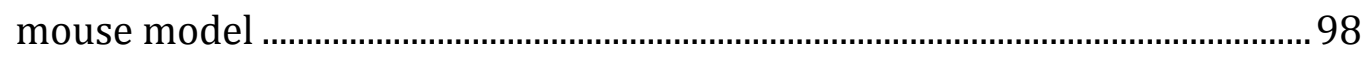

3.13.9 Complete knockout of LPXN using the EIIA-Cre-loxP-system ..........................98

3.13.10 Genotyping of LPXN19 knockout mice................................................................. 100

3.13.11 Phenotypic analysis of LPXN19 knockout mice ................................................ 101

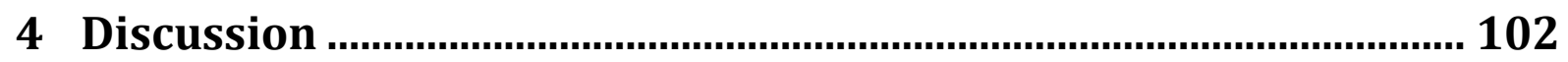

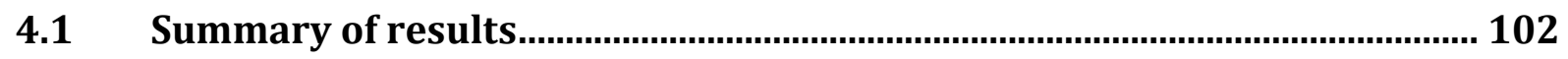


4.2 Paxillin proteins in cytoskeletal rearrangements and migration .............. 104

4.3 Interaction of LPXN and CaD and its implications in prostate cancer...... 108

4.3.1 Caldesmon and its role in cytoskeletal rearrangements ................................ 108

4.3.2 LPXN modulates cytoskeletal rearrangements through interaction

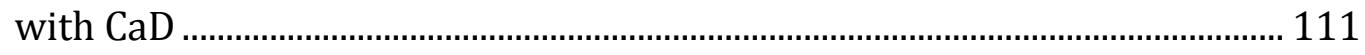

4.4 The role of integrin family proteins in cell adhesion ................................... 114

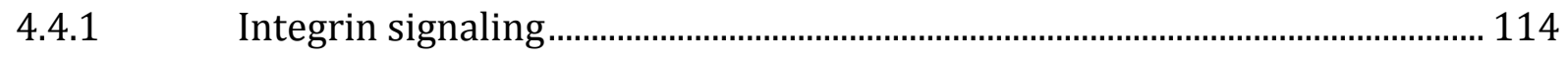

4.4.2 Integrins and their implications in radio- and chemo-resistance ................ 115

4.4.3 LPXN influences radio-resistance through regulation of integrin

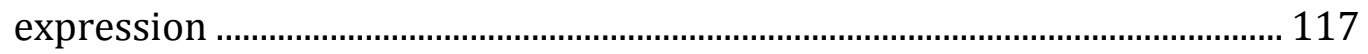

4.5 How does LPXN regulate the expression of the adhesion protein p120-catenin?

4.5.1 The implications of the cell adhesion molecule p120CTN in

LPXN-mediated PCa progression....................................................................... 122

4.5.2 The nuclear function of paxillin family proteins ............................................. 125

4.6 The physiological role of LPXN

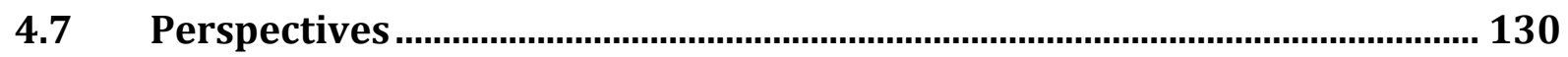

5 Summary ............................................................................................ 134

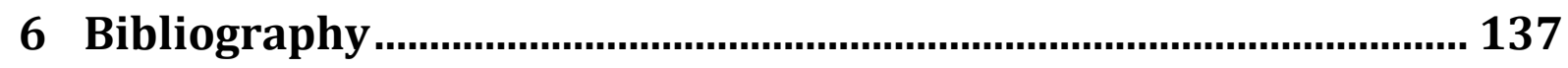

7 Acknowledgements........................................................................... 160 


\section{List of Abbreviations}

\begin{tabular}{|c|c|}
\hline$-m$ & -meter \\
\hline${ }^{\circ} \mathrm{C}$ & Degree Centigrade \\
\hline A & Purinbase Adenin \\
\hline $\mathrm{Ab}$ & Antibody \\
\hline Amp & Ampicillin \\
\hline approx. & Approximately \\
\hline ATP & Adenosine-5'-triphosphate \\
\hline $\mathrm{bp}$ & Base pair(s) \\
\hline BSA & Bovine serum albumine \\
\hline $\mathrm{C}$ & Pyrimidinbase Cytosine \\
\hline cDNA & complementary DNA \\
\hline $\mathrm{Ci}$ & Curie $\left(3.7 \times 10^{10} \mathrm{~Bq}\right)$ \\
\hline $\mathrm{cm}$ & Centimeter \\
\hline DAPI & 4'-6'-Diamidino-2-Phenylindol \\
\hline dATP & Deoxyadenosine-5'-triphosphate \\
\hline dCTP & Deoxycytidine-5'-triphosphate \\
\hline $\mathrm{ddH}_{2} \mathrm{O}$ & bi-destilled water \\
\hline dGTP & Deoxyguanosine-5'-triphosphate \\
\hline DMSO & Demythl Sulfoxide \\
\hline DNA & Deoxyribonucleic acid \\
\hline dNTPs & Deoxynucloeside-5'-phosphate \\
\hline
\end{tabular}




\begin{tabular}{|c|c|}
\hline DTT & Dithiotreitol \\
\hline dTTp & Deoxythymidine-5'-triphosphate \\
\hline E.Coli & Escherichia Coli \\
\hline ECM & Extracellular matrix \\
\hline EDTA & Ethylenediamine tetraacetic acid \\
\hline EGFP & $\begin{array}{l}\text { Enhanced green fluorescent protein } \\
\text { Ethyleneglycol-bis( } \beta \text {-aminoethyle)-N,N,N',N'- }\end{array}$ \\
\hline EGTA & tetraacetic acid \\
\hline EMSA & Electrophoretic mobility shift assay \\
\hline et al. & et alteres \\
\hline etc. & et cetera \\
\hline EtOH & Ethanol \\
\hline Fig. & Figure \\
\hline FITC & Fluorescein isothiocyanate \\
\hline $\mathrm{g}$ & Gram \\
\hline G & Purinbase Guanosin \\
\hline $\mathrm{h}$ & hours \\
\hline $\mathrm{H} / \mathrm{E}$ & Hematoxylin / Eosin \\
\hline Kan & Kanamycin \\
\hline $\mathrm{Kb}$ & Kilobase(s) \\
\hline $\mathrm{KCl}$ & Potassium chloride \\
\hline $\mathrm{kDa}$ & Kilo Dalton \\
\hline 1 & Liter \\
\hline LB & Luria-Bertani \\
\hline LPXN & Leupaxin \\
\hline Luc & Luciferase \\
\hline
\end{tabular}




\begin{tabular}{|c|c|}
\hline M & Molar \\
\hline m- & Milli- \\
\hline $\mathrm{mA}$ & Milliampere \\
\hline $\mathrm{MgCl}_{2}$ & Magnesium chloride \\
\hline $\min$ & minutes \\
\hline $\mathrm{ml}$ & Milliliter \\
\hline $\mathrm{mM}$ & Millimolar \\
\hline $\bmod$ & Modified \\
\hline mRNA & Messegner Ribonucleic acid \\
\hline $\mathrm{n}$ & Nano $=10^{-9}$ \\
\hline $\mathrm{NaCl}$ & Sodium chloride \\
\hline $\mathrm{nm}$ & Nanometer \\
\hline OD & Optical density \\
\hline oligo(dT) & Oligodeoxythymidylic acid \\
\hline $\mathrm{p}$ & pico \\
\hline PAGE & Polyacrylamid gel electrophoresis \\
\hline PBS & Phosphate buffered saline \\
\hline $\mathrm{PCa}$ & Prostate Cancer \\
\hline $\begin{array}{l}\text { PCR } \\
\mathrm{pH}\end{array}$ & $\begin{array}{l}\text { Polymerase chain reaction } \\
\text { negative decimal logarithm of the hydrogen ion } \\
\text { concentration }\end{array}$ \\
\hline PMSF & Phenylmethylsulfonyl fluoride \\
\hline poly (dIdC) & Poly-deoxyinosinic-deoxycytidylic acid \\
\hline RNA & Ribonucleic acid \\
\hline RNAi & RNA interference \\
\hline rpm & Rounds per minute \\
\hline
\end{tabular}




\begin{tabular}{|c|c|}
\hline $\mathrm{RT}$ & Room temperature \\
\hline RT-PCR & Reverse Transcriptase-PCR \\
\hline SDS & Sodium dodecyl sulfate \\
\hline sec & seconds \\
\hline siRNA & small interfering RNA \\
\hline ss & single stranded \\
\hline SV 40 & Simian Virus 40 \\
\hline $\mathrm{T}$ & Pyrimidine base Thymidine \\
\hline Tab. & Table \\
\hline Taq & Thermus aquaticus \\
\hline $\mathrm{U}$ & Unit(s) (Enzymatic activity) \\
\hline UV & Ultraviolett \\
\hline V & Volt \\
\hline Vol. & Volume \\
\hline $\mathrm{w} / \mathrm{o}$ & without \\
\hline $\mathrm{xg}$ & Multiple of acceleration of gravity \\
\hline $\mathrm{X}-\mathrm{Gal}$ & 5-Bromo-4-Chloro-3-Indolyl- $\beta$-D-Galactopyranoside \\
\hline$\mu$ & Micro $=10^{-6}$ \\
\hline$\mu g$ & Microgram \\
\hline$\mu l$ & Microliter \\
\hline$\mu \mathrm{m}$ & Micrometer \\
\hline$\mu \mathrm{M}$ & Micromolar \\
\hline
\end{tabular}




\section{$1 \quad$ Introduction}

\section{$1.1 \quad$ The prostate carcinoma - at a glance}

In 2008 prostate cancer (PCa) was the second most frequently diagnosed cancer and claimed the sixth leading cause of cancer-related death in men worldwide (Global cancer Facts \& Figures 2ndEdt). A majority of these cases (three-quarters) are diagnosed primarily in economically developed countries, but are emerging in developing nations as well. In Germany PCa is the most common cancer type with 65830 (26.1\% of total cancer burden) diagnosed cases in 2010, followed by lung (13.9 \%) and intestinal (13.4\%) cancer. With 12676 (10.8 \% in 2010) deaths it claims the third leading cause of cancer-related death among men in Germany (Krebs in Deutschland 2009/2010, 2013). Therefore, PCa is a serious health problem and a disease of increasing significance both, in Germany and worldwide.

Well-established risk factors for the development of PCa are age, race, and family history. Age clearly is a risk factor, since the ten-year likelihood for a 75-year old man to get PCa is $6 \%$, whereas a man aged 35 has only a $0.1 \%$ risk to develop PCa. A predisposition among close relatives has been documented and might account for $9 \%$ of PCas. However, a distinct genetic mutation has not been reported (Krebs in Deutschland 2009/2010, 2013). In addition, there are some discordant risk factors like diet, hormones and environmental cues that have been subject to extensive studies but did not reveal trusty evidence yet.

Within the last years the prostate cancer incidence has increased constantly. This is mainly due to prostate specific antigen (PSA) testing. The PSA test measures the blood level of PSA, a protein that is exclusively produced in the prostate. These levels can be used as a tumor marker as the blood levels of PSA are increasing with increasing size and growth of the prostate. Therefore, PSA testing detects clinically important tumors, but also those that grow slowly and are not life threatening. However, there are men suffering from PCa that do not show elevated PSA levels and additional reasons for a high PSA level exist. However, PSA testing has been extensively discussed, since a clear correlation of PSA and PCa was not shown so far (Kilpelainen et al. 2011). In addition to this, the PSA test cannot distinguish between locally defined and advanced PCa that would require different therapies. 
The prostate tumor starts to develop as a hormone-dependent lesion and usually is apparent when men become concerned about urinary and sexual dysfunction. This early state tumor is growing slowly and is not life threatening, which is why active surveillance, instead of surgery, irradiation or hormone therapy is the favored treatment option of these tumors. However, a subset of these lesions will progress and result in an advanced setting of prostate cancer, which is characterized by higher invasive and metastatic capacities. Until today there is no effective therapy available for the advanced state of PCa, which is why it is always attended by a poor prognosis for the patient (Trachtenberg, Blackledge 2002; Edwards, Bartlett, John M S 2005). Although many genetic and epigenetic alterations for prostate carcinoma have been proposed, the exact cellular and molecular mechanisms underlying the progression from a locally defined to an advanced prostate carcinoma remain unknown, illustrating the difficulty of diagnosis and the need of new improvements in PCa therapy.

\subsection{Cancer cell migration}

\subsubsection{The essential mechanisms of cancer cell migration}

Metastatic spread, predominantly to the bone marrow, is the main cause of mortality in most prostate cancer patients. This complex process requires a number of genetic events in tumor cells that are incompletely understood. Cells have to invade and migrate through the surrounding tissue, escape from the primary tumor site to the circulation and form metastasis in distant organs. Therefore, a key feature of metastatic spread is cancer cell migration. Cancer cells possess a number of migration and invasion mechanisms, which mainly rely on the regulation of polarization, membrane extensions or protrusions, cell adhesion and cytoskeletal rearrangements (Fig. 1.1). 


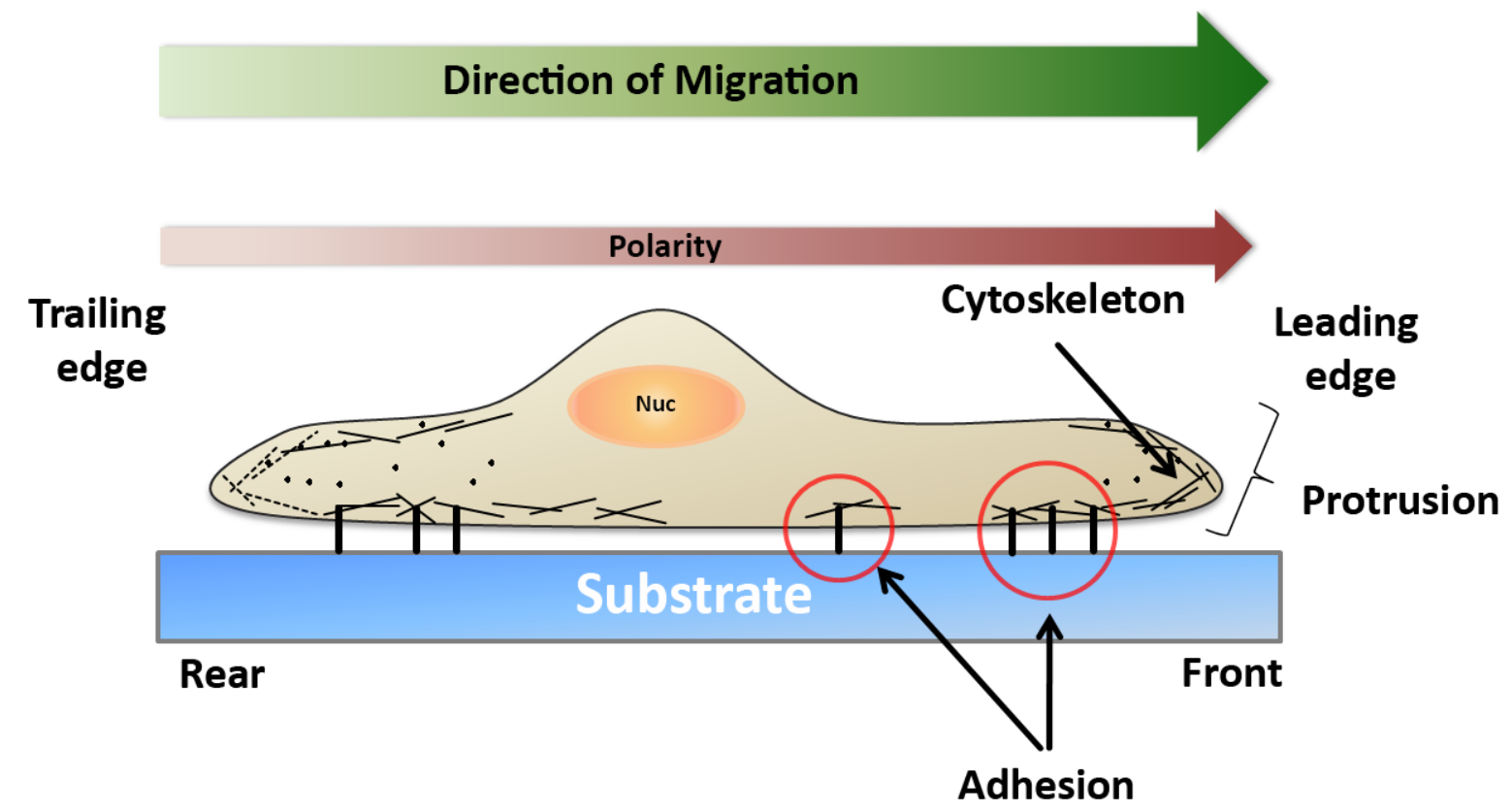

Fig. 1.1: Schematic overview of the processes required for migration. Cell migration relies on several components such as cell polarity, protrusion formation, the formation of new adhesions and cytoskeletal rearrangements that act all together to enable migration. The regulation of these processes is vital for the cell and deregulated in cancer cells. Nuc=Nucleus (Taken and modified from Ridley et al. 2003 and http://www.cellmigration.org).

Cell polarity is a major requirement for the determination of the direction of migration. Environmental cues like chemoattractants or breakdown of cell-cell contacts (wound healing) predetermine this direction of migration and define a cell front and rear. The organization of the cytoskeleton finally establishes a stable polarity of the cell. At the leading edge of the cell a high frequency of actin reassembly is generating membrane protrusions to explore the environment and form focal adhesions. The generation of protrusions requires a scaffold of cytoskeletal structures that is supporting the expansion of the plasma membrane, as well as a contact to the extracellular matrix to provide traction forces for movement. The actin polymerization in these protrusions plays a major role for their stability and turnover. The Arp2/3 complex attaches to the side of already existing actin filaments and starts to add actin monomers resulting in a branched actin network. This actin network connects to the substratum via cell surface receptors of the cadherin and integrin family. Integrins consist of an $\alpha$ - and a $\beta$-subunit, which can form non-covalent heterodimers. They are composed of an extracellular ligand-binding site and a cytoplasmic domain linking the cytoskeletal network to the signal transduction pathways. Interestingly, integrins can mediate bidirectional signaling. On the one hand, intracellular signals can modulate the activity of integrins at 
the plasma membrane (inside-out signaling). On the other hand, signals coming from the cellular environment through ligand-binding of ECM proteins are transmitted to affect intracellular processes (outside-in signaling) (Hynes 2002a; Lau et al. 2009). Upon integrin ligation downstream signaling pathways are activated and focal complexes of a large array of proteins are formed. The mature focal adhesion is composed of kinases and adapter proteins that facilitate signaling through their corresponding pathways. Adapter proteins like Paxillin, ARA55 and Leupaxin, which belong to the paxillin protein family reside at focal adhesion sites and link the cytoskeleton to the extracellular matrix (ECM). These proteins are of major importance for the vital processes of migration, as the loss of intercellular adhesion is a prerequisite for the development and progression of cancer.

Another group of cell surface proteins involved in cell-cell adhesion is the cadherin protein family. Cadherins are trans-membranous proteins composed of an extracellular domain that is binding to cadherins of the neighboring cells and an intracellular domain that associates with catenins to build the basis for the formation of adhesion complexes. Usually cadherins are responsible for cell-cell contacts that are important for maintenance of the tissue structure and prevent motility, which is why cadherins are known as suppressors of invasion and metastasis of epithelial cells (Paredes et al. 2012). Carcinomas derive from epithelial cells and deregulation of E-cadherin is a common feature of these tumor cells.

All these proteins have specific functions in their component processes and act together to establish dynamic contacts between the extracellular environment and the cytoskeleton to generate contractile forces essential for cell migration.

\subsubsection{Cytoskeletal rearrangement during cancer cell migration}

Besides cell adhesion the motility of malignant tumor cells is depending on the stability and assembly of the cytoskeleton. The dynamics of the actin-cytoskeleton are regulated by the small GTPases of the Rho family. These GTPases are known as molecular switches in signaling pathways, as they hydrolyze GTP and are cycling between a GTP-bound (active) and a GDP-bound (inactive) form. The Rho family is divided into several subgroups, among these the Rac, cdc42 and Rho GTPases are the best characterized. These GTPases are master regulators of the actin polymerization to generate stress fibers, filopodia and lamellipodia, respectively. These cytoskeletal structures are 
indispensible for migration. The regulation of small Rho GTPases relies on a variety of activator and repressor proteins that target GTP-hydrolysis.

Another group of proteins that directly binds to actin such as cofilin, filamin or caldesmon regulates the stabilization of the actin cytoskeleton. These proteins facilitate the assembly and disassembly of the cytoskeleton by their affinity to actin. Thus, reduced binding of these actin-stabilizing proteins results in dynamic actin structures and facilitates cytoskeletal remodeling.

The investigation of the cellular and molecular mechanisms involved in cell adhesion and cytoskeletal rearrangements will add to the understanding of how cancer cells can disseminate and will lead to the development of new treatment options.

\section{$1.3 \quad$ Leupaxin (LPXN) - at a glance}

\subsubsection{LPXN, a member of the paxillin protein family}

The LPXN gene is located on chromosome 11q12 and possesses 9 exons, which produces a 386 amino acid protein. Leupaxin (LPXN) was originally identified in cells of hematopoietic origin in 1998 by Lipsky et al. (Lipsky et al. 1998). Due to its high sequence homology to the focal adhesion protein paxillin it was assigned to the paxillin protein family. The paxillin protein family consists of the eponymous paxillin, the androgen receptor-associated protein 55 (ARA55) formerly known as hydrogen peroxideinducible clone 5 (HIC-5) and leupaxin (LPXN). Proteins of the paxillin protein family are characterized by their subcellular localization at focal adhesion sites. Focal adhesion sites are macromolecular assemblies of a large array of proteins that exhibit multiple protein-protein interactions including structural or membrane-spanning proteins that bind to their ligands in the extracellular matrix (ECM) as well as signaling proteins that mediate regulatory effects of ECM adhesion (Turner 2000).

Another characteristic of the paxillin protein family is the presence of a multiple of two highly conserved domain structures. The four (ARA55 and leupaxin) or rather five (paxillin) LD motifs at the N-terminal region of the proteins are composed of a leucinerich amino acid sequence with an invariant leucine (L)-aspartic acid (D) pair. The Cterminal region harbors four approximately 55 amino acid long LIM domains, which contain conserved cysteine, histidine and aspartic acid residues. The LIM domain was first discovered in the homeobox-transcription factors Lin-11, Isl-1 and Mec-3 (=LIM) in 
Caenorhabditis elegans (Way, Chalfie 1988). This protein domain displays highly conserved residues that guarantee the formation of a tandem zinc finger motif, which seems to be essential for secondary structure formation (Kadrmas, Beckerle 2004). Usually zinc finger motifs are present in transcription factors and known to facilitate DNA binding. However, the function of LIM domains was long time believed to be restricted to protein-protein-interactions, but the presence of a LIM domain is emerging as a hallmark of proteins that can associate with the actin cytoskeleton, the transcriptional machinery or directly with DNA (Nishiya et al. 1998; Muller et al. 2002).

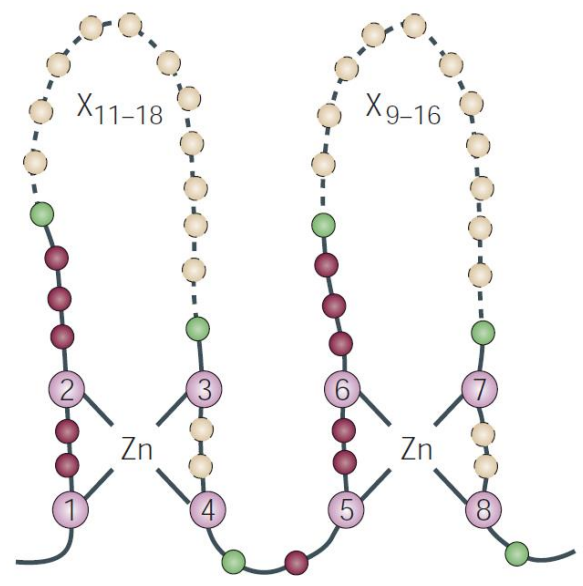

Fig. 1.1: Tandem zinc finger motif of LIM domains. Highly conserved residues generate a tandem zinc finger motif with a spacer of two amino acids. The formation of a tandem zinc finger motif seems to be essential for proper LIM domain function. Usually, zinc finger motifs are found in DNA binding proteins. Taken from (Kadrmas, Beckerle 2004).

For ARA55 DNA binding via its LIM domains was described. This binding turned out to require zinc for the proper formation of the LIM domain. Each of the four LIM domains contributed to DNA binding of a poly(A) stretch such as those present in the 3 '-end of mRNAs. Since, a consensus binding sequence was not found and a target gene was not described, ARA55 was suggested to associate with secondary DNA structures to exert its nuclear function. For paxillin it was shown that LD-motifs as well as LIM domains are important for protein-protein-interactions and localization to focal adhesion sites (Brown et al. 1996; Brown et al. 1998; Dawid et al. 1998). In addition, the LIM domains of LPXN were described to be similar in focal adhesion targeting by tyrosine phosphorylation but were distinct from paxillin function in cell adhesion and spreading (Chen, Kroog 2010).

In focal adhesions (FAs) paxillin proteins have various functions. Paxillin for instance binds to the cytoplasmic tail of several integrins and is thereby able to mediate signals 
from the cellular environment into the cell through direct or indirect interaction with structural or signaling molecules like vinculin, PTP-PEST or the focal adhesion kinase (FAK) (Shen et al. 1998; Turner 2000). In addition, Paxillin is able to regulate the rhofamily GTPases, which are major players of actin-cytoskeleton and adhesion dynamics (Petit et al. 2000).

\subsection{The function of LPXN}

In contrast to paxillin, there is only little evidence about the function of LPXN today and contradictory results are making the situation more complicated. Originally, LPXN was found to be expressed in hematopoietic cells (Lipsky et al. 1998). In this publication LPXN was described to form a complex with the non-receptor tyrosine kinase PYK2, belonging to the FAK family of kinases, to influence cell motility, spreading and apoptosis. In osteoclasts, LPXN associates with the sarcoma kinase, SRC, and is involved in bone resorption. In addition, LPXN localizes to the podosomal/sealing zone complex, which in other cells is known as focal adhesion (Gupta et al. 2003). In B-cells, LPXN plays an inhibitory role. Interaction of LPXN and LYN, a critical SRC family tyrosine kinase in B-cell receptor (BCR) signaling, is induced by BCR stimulation resulting in a suppression of BCR signaling (Chew, Lam 2007). Initially, LPXN was described as a cytoplasmic protein residing at focal adhesions. In rodent aortic smooth muscle cells LPXN interacts with the (FAK) to form a complex that attenuates nuclear accumulation of LPXN. In the nucleus LPXN functions as a serum response factor (SRF) cofactor to induce smooth muscle differentiation-marker gene expression (Sundberg-Smith et al. 2008). Obviously, LPXN is able to shuttle between the nuclear and cytoplasmic compartment. Consequently, LPXN might be more than a focal adhesion protein and takes part in processes beyond FA formation.

The only clinically relevant observation of a LPXN mutation until today was an acute myeloid leukemia (AML) patient who was carrying a translocation $(\mathrm{t}(11 ; 21)(\mathrm{q} 12 ; \mathrm{q} 22))$. This translocation fused the RUNX1 gene with the LPXN gene, which produced a RUNX1/LPXN fusion protein. Both proteins, wild type RUNX1 as well as the RUNX1/LPXN fusion protein were present in the nuclei of hematopoietic cells, where they were competing for the binding site in the promoter of colony-stimulating factor 1 receptor (CSF1R). Consequently, CSF1R expression was reduced, which resulted in disturbed proliferation and differentiation of the cells. Interestingly, subcutaneous 
injection of pEGFP-N1-RUNX1/LPXN stably transfected NIH/3T3 cells into BALB/c nude mice resulted in development of carcinomas after 28 days (Dai et al. 2009). This study is providing in vitro evidence that LPXN might display a candidate gene associated with carcinogenesis. However, despite the functions mentioned above, only little is known about the physiological role of LPXN. Especially the molecular mechanisms of LPXN mediated processes are incompletely understood.

\subsection{The function of LPXN in prostate cancer}

First expression of LPXN in PCa was noticed as an Atlas ${ }^{\mathrm{TM}}$-array hybridization was conducted on micro-dissected human prostate carcinoma specimen in our research group (Voigt, 2003). Further studies confirmed focal overexpression exclusively in epithelial carcinoma cells in $22 \%$ of prostate carcinomas analyzed and showed that LPXN expression was correlating with the gleason score of the corresponding carcinoma (Kaulfuss et al. 2008). These findings were supported by western blot analysis of LPXN expression in the PCa cell lines PC-3, DU145 and LNCaP, which showed that cell lines that represented the advanced setting of PCa (PC-3 and DU145) displayed higher expression levels of LPXN than non-invasive and androgen-dependent LNCaP cells (Kaulfuss et al. 2008). Moreover, LPXN was influencing the migration and invasion of PCa cells, especially of the invasive cell lines PC-3 and DU145. Downregulation of LPXN reduced the invasion of these cells and also a significant reduction in migration was observed (Kaulfuß, 2006). Overexpression of LPXN had promoting effects on migration and invasion in PC-3 cells (Beckemeyer, 2007). Interestingly, we could show that LPXN shuttles between the cytoplasm/membrane and the nucleus and is able to transactivate the androgen receptor. This activation results in increased transcription of androgenresponsive genes such as PSA (Kaulfuss et al. 2008).

The involvement of LPXN in PCa progression was further strengthened by in vivo studies using the transgenic adenocarcinoma of the mouse prostate (TRAMP) mouse model. These TRAMP mice were bred with a mouse model that specifically overexpressed LPXN in the prostate. Double transgenic TRAMP/LPXN-mice resulting from this mating showed increased numbers of poorly differentiated PCa and distant metastasis as compared to control TRAMP mice. Using murine primary tumor cells isolated from the prostate of double transgenic TRAMP/LPXN-mice it was shown that LPXN is enhancing the invasion and migration abilities of these cells (Kaulfuß et la. 2009, von Hardenberg, 
2010). In addition, LPXN-mediated invasion of PCa cells was described to involve the cell adhesion molecule p120catenin (p120CTN), which displayed negative correlation with LPXN expression. p120CTN is a major component of the adhesion complex, which also contains $\beta$-catenin. The expression of $\mathrm{p} 120 \mathrm{CTN}$ has dramatic effects on the stabilization of the adhesion complexes. As mentioned earlier cell adhesion is a critical feature of cell migration and is severely affected by the stabilization of the adhesion complex. Overexpression of LPXN causes p120CTN expression to decrease, leading to a destabilization of the adhesion complexes, to the reduction of cell-cell contacts and subsequent accumulation of $\beta$-catenin in the nucleus. The induction of $\beta$-catenin target gene expression such as matrix metalloproteases (e.g. MMP-7) finally leads to degradation of the extracellular matrix and will enable invasion into the surrounding tissue (Kaulfuss et al. 2009). The expression of multiple matrix metalloproteases is a known hallmark of tumor cell invasion (Ellerbroek, Stack 1999; Stetler-Stevenson 2001). This mechanism is leaving an explanation for the invasive growth of PCa with LPXN overexpression. However, the molecular mechanism that drives LPXN-mediated migration of PCa cells still remained elusive.

To investigate how LPXN might influence prostate cancer cell migration, a yeast-2hybrid screening was conducted on a prostate specific cDNA library, which identified the actin-binding protein caldesmon (CaD) as a putative interaction partner of LPXN (von Hardenberg, 2007). CaD was demonstrated to reduce invasion of breast and intestinal tumor cells by regulation of podosome and invadopodia formation (Yoshio et al. 2007). In addition, CaD was shown to regulate cell growth and survival of vascular smooth muscle cells via the cytoskeletal tension-FAK-ERK1/2 axis (Yokouchi et al. 2006). As a protein that is implicated in cytoskeletal rearrangements CaD might display a putative candidate to execute LPXN-associated changes in migration of PCa cells. The elucidation of the molecular mechanisms underlying the LPXN-mediated progression of PCa might be of great value for patients with advanced prostate carcinoma and might also open new avenues for therapeutic intervention.

\subsection{Aims of this study}

In the present thesis the molecular mechanisms of LPXN-mediated PCa progression are investigated. Based on previous findings the underlying mechanism of LPXN-mediated 
migration of PCa cells is elucidated. In addition, not only the pathological but also the physiological role of LPXN is subject of the thesis.

The main aims were:

- $\quad$ Analyses on the putative LPXN interaction partner CaD and its effect on PCa cell migration

○ Confirmation of the interaction of $\mathrm{CaD}$ and LPXN in prostate cancer cells

- Analyses of the interaction of $\mathrm{CaD}$ and LPXN by co-immunoprecipitation

$\circ \quad$ Analyses of the interaction of $\mathrm{CaD}$ and LPXN by in situ proximity ligation assay (PLA)

- $\quad$ Analyses on the interaction of CaD and LPXN during PCa cell migration

○ Analyses on the putative LPXN interaction partner ERK during PCa cell migration

- $\quad$ Analysis on the influence of LPXN on adhesion of PCa cells

- Analysis on the influence of LPXN on the expression of the adhesion molecule p120CTN

○ Cloning of p120CTN promoter fragments into the reporter plasmid pGL4.16 luc2CP/Hygro

- Identify LPXN responsive elements in the p120CTN promoter region using luciferase reporter gene assays

○ Identify LPXN binding sites in the p120CTN promoter region using EMSA

- $\quad$ Analysis on the influence of LPXN on the expression of $\beta 1$ integrin (ITGB1)

○ $\quad$ Analysis of $\beta 1$ integrin (ITGB1) expression after LPXN knockdown

○ Analysis of $\beta 1$ integrin (ITGB1) expression after LPXN overexpression

- $\quad$ Analysis of LPXN-mediated radio-resistance of PCa cells

○ Analysis on the survival of PCa cells after LPXN knockdown and exposure to ionizing irradiation

- Analysis on the survival of PCa cells after inhibition of $\beta 1$ integrin (ITGB1) and exposure to ionizing irradiation 
- $\quad$ Analysis on the effect of ionizing irradiation on LPXN expression

○ $\quad$ Analysis of LPXN expression in PCa cells after exposure to ionizing irradiation

- Analysis on the effect of ionizing irradiation on ITGB1 expression

- Analysis of $\beta 1$ integrin (ITGB1) expression in PCa cells after exposure to ionizing irradiation

- $\quad$ Generation of a conditional knockout mouse model for LPXN

- Purchase of an ES cell clone with a LPXN targeted trap construct

- Excision of the reporter/selector cassette to generate a conditional allele

- Blastocyst injection of mutant ES cells

○ Breeding of chimera to establish a conditional knockout mouse model for LPXN 


\section{$2 \quad$ Materials and Methods}

\subsection{Chemicals and Reagents}

\begin{tabular}{|c|c|}
\hline Chemicals & Manufacturer \\
\hline $\begin{array}{l}\text { 5-Bromo-4-Chloro-3-indolyl- } \alpha \text {-D- } \\
\text { galactopyranosid(X- } \alpha \text {-Gal) }\end{array}$ & Carl Roth GmbH, Karlsruhe, Germany \\
\hline Adenosintriphosphate (ATP) & Biomol GmbH, Hamburg, Germany \\
\hline Agar & Carl Roth GmbH, Karlsruhe, Germany \\
\hline Agarose & Life Technolgies, Darmstadt, Germany \\
\hline Ammonium persulfate (APS) & Carl Roth GmbH, Karlsruhe, Germany \\
\hline Ampicillin & Carl Roth GmbH, Karlsruhe, Germany \\
\hline Ampuwa & Fresenius AG, Bad Homburg, Germany \\
\hline Bacto-Trypton & Carl Roth GmbH, Karlsruhe, Germany \\
\hline Borica cid & Scharlau Chemie S.A., Barcelona, Spain \\
\hline Cell culture media & PAN, Aidenbach, Germany \\
\hline Dimethylsulfoxide & Carl Roth GmbH, Karlsruhe, Germany \\
\hline Dithiotreitol (DTT) & Biomol GmbH, Hamburg, Germany \\
\hline DNA Stain G & Serva GmbH, Heidelberg, Germany \\
\hline dNTPs (100 mM) & Life Technolgies, Darmstadt, Germany \\
\hline Ethanol & $\begin{array}{l}\text { Chemie Vertrieb Hannover, Hannover, } \\
\text { Germany }\end{array}$ \\
\hline Ethidiumbromid & Sigma-Aldrich, Deisenhofen, Germany \\
\hline Ethylendiamine-tetraacetic acid (EDTA) & ICN, Aurora, USA \\
\hline $\begin{array}{l}\text { Ethylenglycol-bis( } \beta \text {-aminoethyl)-N,N,N',N'- } \\
\text { tetraacetat (EGTA) }\end{array}$ & Carl Roth GmbH, Karlsruhe, Germany \\
\hline Ficoll ${ }^{\circledR} 400$ & $\begin{array}{l}\text { AppliChem GmbH, Darmstadt, } \\
\text { Germany }\end{array}$ \\
\hline Formaldehyde & Carl Roth GmbH, Karlsruhe, Germany \\
\hline Glycerol & Carl Roth GmbH, Karlsruhe, Germany \\
\hline
\end{tabular}




\begin{tabular}{|c|c|}
\hline Glycin & | Carl Roth GmbH, Karlsruhe, Germany \\
\hline Igepal CA-630 (NP-40) & Sigma-Aldrich, Deisenhofen, Germany \\
\hline Isopropanol & Carl Roth GmbH, Karlsruhe, Germany \\
\hline Kanamycin & Sigma-Aldrich, Deisenhofen, Germany \\
\hline Magnesium chloride hexahydrate & Carl Roth GmbH, Karlsruhe, Germany \\
\hline Methanol & Carl Roth GmbH, Karlsruhe, Germany \\
\hline $\begin{array}{l}\text { N,N,N',N'-Tetramethylethylenediamine } \\
\text { (TEMED) }\end{array}$ & Carl Roth GmbH, Karlsruhe, Germany \\
\hline NuPAGE $^{\mathrm{TM}}$ LDS Sample buffer (4x) & Life Technolgies, Darmstadt, Germany \\
\hline NuPAGE $^{\text {TM }}$ MES Running buffer (20x) & Life Technolgies, Darmstadt, Germany \\
\hline NuPAGE $^{\text {tM }}$ See Blue Plus2 & Life Technolgies, Darmstadt, Germany \\
\hline Orange G & Sigma-Aldrich, Deisenhofen, Germany \\
\hline PD98059 (MEK1 Inhibitor) \#9900S & Cell Signaling, Danvers, MA, USA \\
\hline Penicillin/Streptomycin & PAN, Aidenbach, Germany \\
\hline Phenylmethylsulfonyl flouride (PMSF) & Sigma-Aldrich, Deisenhofen, Germany \\
\hline $\begin{array}{l}\text { Poly(deoxyinosinic-deoxycytidylic) acid } \\
\text { sodiumsalt }\end{array}$ & Sigma-Aldrich, Deisenhofen, Germany \\
\hline Potassium chloride & Carl Roth GmbH, Karlsruhe, Germany \\
\hline RNase inhibitor & MBI, St. Leon-Rot, Germany \\
\hline Roti ${ }^{\circledR}$ Nanoquant & Carl Roth GmbH, Karlsruhe, Germany \\
\hline Roti ${ }^{\circledR}$ Phorese (29:1) & Carl Roth GmbH, Karlsruhe, Germany \\
\hline $\begin{array}{l}\text { Simply Blue Safe Stain } \\
\text {. }\end{array}$ & Life Technolgies, Darmstadt, Germany \\
\hline Sodiumdodecylsulfate (SDS) & Serva GmbH, Heidelberg, Germany \\
\hline Tris & $\begin{array}{l}\text { AppliChem GmbH, Darmstadt, } \\
\text { Germany }\end{array}$ \\
\hline Triton X-100 & Fluka, Deisenhofen, Germany \\
\hline Tween 20 & Merck, Darmstadt, Germany \\
\hline Vectashield with DAPI & VectorLab, Burlingame, USA \\
\hline Yeast extract & Carl Roth GmbH, Karlsruhe, Germany \\
\hline$\alpha$-P33-Deoxyadenosine 5'-triphosphate (dATP) & $\begin{array}{l}\text { Hartmann Analytic GmbH, } \\
\text { Braunschweig, Germany }\end{array}$ \\
\hline
\end{tabular}




\subsection{Biochemicals and enzymes}

\begin{tabular}{|c|c|}
\hline Biochemical & Manufacturer \\
\hline Albumin fraction $\mathrm{V}$ & Biomol GmbH, Hamburg, Germany \\
\hline BigDye $^{\circledR}$ & Life Technologies, Darmstadt, Germany \\
\hline $\begin{array}{l}\text { Complete Mini Protease Inhibitor Cocktail } \\
\text { Tablets }\end{array}$ & Roche, Mannheim, Germany \\
\hline Direct PCR Lysis Reagent & Peqlab, Erlangen, Germany \\
\hline DNAse & Sigma-Aldrich, Deisenhofen, Germany \\
\hline Fetal bovine serum (SeraPlus) & PAN, Aidenbach, Germany \\
\hline MangoTaq-DNA-Polymerase & Bioline, Luckenwalde, Germany \\
\hline MycoZAP $^{\mathrm{TM}}$ Spray & Lonza, Cologne, Germany \\
\hline Phalloidin, FITC-labeled & Sigma-Aldrich, Deisenhofen, Germany \\
\hline Phosphatase Inhibitor Mix II, solution & Serva GmbH, Heidelberg, Germany \\
\hline Phusion $^{\mathrm{TM}}$ High-Fidelity DNA Polymerase & Finnzymes, Espoo, Finland \\
\hline Proteinase $\mathrm{K}$ & Carl Roth GmbH, Karlsruhe, Germany \\
\hline Restriction Enzymes & New England Biolabs, Ipswich, USA \\
\hline Reverse Transcriptase SuperScript II & Life Technologies, Darmstadt, Germany \\
\hline RNase A & AppliChem GmbH, Darmstadt, Germany \\
\hline T4 DNA Ligase & Life Technologies, Darmstadt, Germany \\
\hline Trypsin/EDTA solution & PAN, Aidenbach, Germany \\
\hline $\begin{array}{l}\text { Human Epidermal Growth Factor (EGF) } \\
\text { \#8916SF }\end{array}$ & Cell Signaling, Danvers, MA, USA \\
\hline DNA Polymerase I, Klenow-fragment & New England Biolabs, Ipswich, USA \\
\hline
\end{tabular}




\section{$2.3 \quad$ Usage ware}

\begin{tabular}{|c|c|}
\hline Usageware & Manufacturer \\
\hline $10,13,50 \mathrm{ml}$ Cellstar ${ }^{\circledR}$ Tubes & Greiner-bio-one, Kremsmünster, Austria \\
\hline 384-well plates, white & ABgene, Hamburg, Germany \\
\hline \multirow{2}{*}{ 6-, 24-well cell culture plates } & Sarstedt, Nürnbrecht, Germany \\
\hline & Corning Inc., New York, USA \\
\hline Blotting Paper GB 002, 003, 004 & Schleicher \&Schnüll, Dassel, Germany \\
\hline Cell culture flasks & Sarstedt, Nürnbrecht, Germany \\
\hline Coverglass $24 \times 60 \mathrm{~mm}$ & Menzel Gläser, Braunschweig, Germany \\
\hline FALCON culture slides & $\begin{array}{l}\text { Becton Dickinson GmbH, Heidelberg, } \\
\text { Germany }\end{array}$ \\
\hline $\begin{array}{l}\text { Flat-bottomed Nuclon }{ }^{\mathrm{TM}} \text { surface } 96 \text {-well cell } \\
\text { culture plates }\end{array}$ & Nunc A/S, Danmark \\
\hline Membrane filter & Millipore, Billerica, USA \\
\hline Microcentrifuge Tubes & Sarstedt, Nürnbrecht, Germany \\
\hline NuPAGE $^{\mathrm{TM}} 4$ - $12 \%$ Bis-Tris Gels & Life Technologies, Darmstadt \\
\hline Petri dishes & Greiner Nunc., Nürntingen, Germany \\
\hline Pipet tips & Sarstedt, Nürnbrecht, Germany \\
\hline PVDF-Membrane & GE Healthcare, Munich, Germany \\
\hline Quarz-Cuvette & Hellma, Mühlheim, Germany \\
\hline Sterile Single-use filter Minisart & Sartorius, Göttingen, Germany \\
\hline Nunc $^{\circledR}$ F96 MicroWell ${ }^{\text {TM }}$ white & Nunc A/S, Danmark \\
\hline
\end{tabular}

\section{$2.4 \quad$ Technical equipment}

\begin{tabular}{l|l}
\hline \multicolumn{1}{c|}{ Technical equipment } & \multicolumn{1}{c}{ Manufacturer } \\
\hline FluorChem ${ }^{\circledR} \mathrm{Q}$ & Alpha Innotech, Logan, Utah, USA \\
\hline Confocal Laser Scanning Microscope IX81 & Olympus, Hamburg, Germany \\
\hline SynergyMx & Bio Tek, Bad Friedrichshall, Germany \\
\hline Irradiation device 225A & Gulmaymedical, Camberley, UK \\
\hline Fujifilm FLA-5100 & Fuji Life Science, Düsseldorf, Germany \\
\hline
\end{tabular}


HT7900 Fast Real-Time PCR System

Applied Biosystems GmbH, Darmstadt,
Germany

\subsection{Sterilization of solution and equipment}

Laboratory equipment, solutions and culture media were autoclaved at $121{ }^{\circ} \mathrm{C}$ and 105 Pa for 60 min or sterilized at $220^{\circ} \mathrm{C}$ over night.

\section{$2.6 \quad$ Ready-to-use Reaction systems}

\begin{tabular}{|c|c|}
\hline Reaction system & Manufacturer \\
\hline Direct PCR tail reagent & Peqlab, Erlangen, Germany \\
\hline ECL Prime & GE Healthcare, Munich, Germany \\
\hline Myco Alert@ Mycoplasma Detection Kit & Lonza, Cologne, Germany \\
\hline peqGold Total RNA Kit & Peqlab, Erlangen, Germany \\
\hline NucleoSpin ${ }^{\circledR}$ Extract II & Macherey \& Nagel, Düren, Germany \\
\hline Plasmid Midi Kit & Life Technologies, Darmstadt, Germany \\
\hline $\begin{array}{l}\text { Platinum }{ }^{\circledR} \text { SYBR }{ }^{\circledR} \text {-Green qPCR Super Mix- } \\
\text { UDG }\end{array}$ & Life Technologies, Darmstadt, Germany \\
\hline PhosSTOP Phosphatase Inhibitor & Roche, Mannheim, Germany \\
\hline Duolink ${ }^{\circledR}$ in situ Kit & Olink Bioscience, Uppsala, Sweden \\
\hline MSB $^{\circledR}$ Spin PCR apace & Invitek, Berlin, Germany \\
\hline MicroSpin Columns & GE Healthcare, Munich, Germany \\
\hline
\end{tabular}

\section{$2.7 \quad$ Solutions}

Solutions for routine applications were prepared according to (Maniatis et al. 1982). Required chemicals were dissolved in $\mathrm{ddH}_{2} \mathrm{O}$ and autoclaved or filtered under sterile conditions when necessary. 


\begin{tabular}{|c|c|}
\hline \multirow[t]{3}{*}{ AP Buffer } & $100 \mathrm{mM} \mathrm{NaCl}$ \\
\hline & $50 \mathrm{mM} \mathrm{MgCl} 2$ \\
\hline & 100 mM Tris/HCl pH 9.5 \\
\hline \multirow[t]{3}{*}{ AP Staining Solution } & $45 \mu \mathrm{l}$ NBT $(75 \mathrm{mg} / \mathrm{ml}$ in DMF) \\
\hline & $35 \mu \mathrm{l}$ BCIP $(50 \mathrm{mg} / \mathrm{ml}$ in DMF) \\
\hline & in $10 \mathrm{ml}$ AP-Buffer \\
\hline \multirow[t]{2}{*}{ Blocking Buffer (Western Blot) } & 1x TBS-Tween \\
\hline & $5 \%$ low-fat dry milk \\
\hline \multirow[t]{2}{*}{ Blocking Buffer (PLA) } & $1 \mathrm{x}$ PBS \\
\hline & $3 \%$ BSA \\
\hline \multirow[t]{3}{*}{ Coomassie Staining Solution } & $30 \%(\mathrm{v} / \mathrm{v})$ Methanol \\
\hline & $10 \%(\mathrm{v} / \mathrm{v})$ Glacialacetic acid \\
\hline & $0.05 \%(\mathrm{w} / \mathrm{v})$ Coomassie Brilliant Blue R250 \\
\hline \multirow[t]{7}{*}{ Cytoplasmic Extraction Buffer } & 20 mM HEPES pH 7.4 \\
\hline & $10 \mathrm{mM} \mathrm{KCl}$ \\
\hline & $10 \%(\mathrm{v} / \mathrm{v})$ Gylcerol \\
\hline & $1 \mathrm{mM}$ EDTA \\
\hline & $0.1 \%$ IGEPAL-CA-360 (NP-40) \\
\hline & $3 \mathrm{mM}$ DTT \\
\hline & $\begin{array}{l}1 \text { Tablet/ } 10 \mathrm{ml} \text { Complete Mini protease } \\
\text { inhibitor }\end{array}$ \\
\hline \multirow[t]{6}{*}{ EMSA Shift Buffer } & 100 mM HEPES, pH 7,9 \\
\hline & $200 \mathrm{mM} \mathrm{KCl}$ \\
\hline & $5 \mathrm{mM} \mathrm{MgCl}_{2}$ \\
\hline & $20 \%$ Ficoll \\
\hline & 0.5 mM EGTA \\
\hline & $2.5 \mathrm{mM}$ EDTA \\
\hline \multirow[t]{4}{*}{ EMSA Running Buffer Stock Solution } & $1 \mathrm{x}$ TBE \\
\hline & $88 \mathrm{mM}$ Boric acid \\
\hline & $2 \mathrm{mM}$ EDTA \\
\hline & 140 mM Tris pH 8.0 \\
\hline $\begin{array}{l}\text { Lysis Buffer for protein (modified } \\
\text { RIPA) }\end{array}$ & $150 \mathrm{mM} \mathrm{NaCl}$ \\
\hline
\end{tabular}




\begin{tabular}{|c|c|}
\hline & $1 \mathrm{mM}$ EDTA \\
\hline & 50 mM Tris-HCl, pH 7.4 \\
\hline & $1 \%$ IGEPAL-CA-360 (NP-40) \\
\hline & $0.25 \%$ Natriumdeoxycholat \\
\hline & $\begin{array}{l}1 \text { Tablet/10 ml Complete Mini protease } \\
\text { inhibitor }\end{array}$ \\
\hline & $\begin{array}{l}100 \mu \mathrm{l} / 10 \mathrm{ml} \text { Phosphatase-Inhibitor-Mix II, } \\
\text { solution }\end{array}$ \\
\hline \multirow[t]{6}{*}{ Nuclear Extraction Buffer } & 20 mM HEPES pH 7.4 \\
\hline & $420 \mathrm{mM} \mathrm{KCl}$ \\
\hline & $20 \%(\mathrm{v} / \mathrm{v})$ Glycerol \\
\hline & $1 \mathrm{mM}$ EDTA \\
\hline & $3 \mathrm{mM}$ DTT \\
\hline & $\begin{array}{l}1 \text { Tablet/10 ml Complete Mini protease } \\
\text { inhibitor }\end{array}$ \\
\hline \multirow[t]{3}{*}{ P1 Buffer (Plasmid Preparation) } & 50 mM Tris-HCl, pH 8.0 \\
\hline & $10 \mathrm{mM}$ EDTA \\
\hline & $100 \mu \mathrm{g} / \mathrm{ml}$ RNase $\mathrm{A}$ \\
\hline \multirow[t]{2}{*}{ P2 Buffer (Plasmid Preparation) } & $200 \mathrm{mM} \mathrm{NaOH}$ \\
\hline & $1 \%$ SDS \\
\hline P3 Buffer (Plasmid Preparation) & 3 M Potassium acetate $(\mathrm{pH} 5.5)$ \\
\hline \multirow[t]{2}{*}{ Potassium Phosphate (1M) } & $0.17 \mathrm{KH}_{2} \mathrm{PO}_{4}$ \\
\hline & $0.72 \mathrm{~K}_{2} \mathrm{HPO}_{4}$ \\
\hline \multirow[t]{4}{*}{ 10x PBS } & $1.37 \mathrm{M} \mathrm{NaCl}$ \\
\hline & $81 \mathrm{mM} \mathrm{Na}_{2} \mathrm{PO}_{4}$ \\
\hline & $27 \mathrm{mM} \mathrm{KCl}$ \\
\hline & $14.7 \mathrm{mM} \mathrm{KH}_{2} \mathrm{PO}_{4}$ \\
\hline \multirow[t]{4}{*}{ Stop Mix 1} & $95 \%$ Formamide \\
\hline & $20 \mathrm{mM}$ EDTA \\
\hline & $0.05 \%$ Bromphenolblau \\
\hline & $0.05 \%$ Xylencyanol \\
\hline \multirow[t]{2}{*}{ Stop Mix 2} & $15 \%$ Ficoll 400 \\
\hline & $200 \mathrm{mM}$ EDTA \\
\hline
\end{tabular}




\begin{tabular}{l|l} 
& $0.1 \%$ Orange G \\
\hline $10 x$ TBS & $1.37 \mathrm{M} \mathrm{NaCl}$ \\
\hline $1 \mathrm{x}$ TBS-Tween (TBS-T) & $100 \mathrm{mM}$ Tris \\
\hline & Adjusted to $\mathrm{pH} 7.6 \mathrm{using} \mathrm{HCl}$ \\
\hline Transfer Buffer IIa (Western Blot) & $1 \mathrm{x}$ TBS \\
\hline & $0.1 \%$ Tween 20 \\
\hline 5x Tris-Boricacid-EDTA (TBE) Buffer & $445 \mathrm{mM}$ Tris/HCl, pH 8.0 \\
\hline & $150 \mathrm{mM}$ Glycin 8.3 \\
\hline 20x Turbo Buffer & $20 \% \mathrm{mM}$ Boricacid \\
\hline & $10 \mathrm{mM}$ EDTA \\
\hline Washing Buffer (Western Blot) & $1 \mathrm{x}$ TBS-Tween \\
\hline & $2.5 \%$ low-fat dry milk \\
\hline Xal Stock solution & $20 \mathrm{mg}$ X-Gal/ml N.N.-Dimethyl-foramide \\
\hline
\end{tabular}

\section{$2.8 \quad$ Culture media, antibiotics, agar plates}

\subsubsection{Culture media for bacteria}

Luria-Bertani medium (LB medium)

$$
\begin{aligned}
& \text { 10g/l Bacto-Trypton } \\
& 5 \mathrm{~g} / \mathrm{l} \mathrm{Yeast} \mathrm{Extract} \\
& 10 \mathrm{~g} / \mathrm{l} \mathrm{NaCl} \\
& \mathrm{pH} 7.0
\end{aligned}
$$

The culture medium was prepared using bi-destilled water, autoclaved and kept at $4{ }^{\circ} \mathrm{C}$. For selection ampicillin (50 $\mu \mathrm{g} / \mathrm{ml}$ final concentration) or kanamycin $(25 \mu \mathrm{g} / \mathrm{ml}$ final concentration) were added to the medium, respectively. 


\subsubsection{Agar plates}

Before autoclaving $1.5 \%(\mathrm{w} / \mathrm{v})$ Agar-Agar was added to the liquid LB medium. After the medium was autoclaved it was cooled down to $55^{\circ} \mathrm{C}$ on a stirring plate before antibiotics were added in a corresponding concentration (ampicillin: $50 \mu \mathrm{g} / \mathrm{ml}$, kanamycin: $25 \mu \mathrm{g} / \mathrm{ml}$ ). Finally, the medium was poured into petri dishes and kept in a sterile plastic bag at $4^{\circ} \mathrm{C}$.

\subsubsection{Culture media for eukaryotic cell cultures}

Media used for culture of eukaryotic cells were purchased from PAN, Aidenbach, Germany. Before use fetal bovine serum (FBS) and antibiotics (penicillin/streptomycin) were added. For cell culture the following media were used:

Medium for PC-3, DU145 and LNCaP cells:

RPMI 1640 (PAN, Aidenbach, Germany)

$100 \mu \mathrm{g} / \mathrm{ml}$ Streptomycin

100 U/ml Penicillin

$10 \%$ fetal bovine serum (FBS)

Medium for HeLa cells:

$$
\begin{aligned}
& \text { MEM (PAN, Aidenbach, Germany) } \\
& 100 \text { Hg/ml Streptomycin } \\
& 100 \mathrm{U} / \mathrm{ml} \text { Penicillin } \\
& 10 \% \text { fetal bovine serum (FBS) }
\end{aligned}
$$

ES cell medium:

Dulbecco's MEM (DMEM) (Life Technologies, Germany)

$0.1 \mathrm{mM} \quad$ Non essential amino acids

$1.0 \mathrm{mM} \quad$ Sodium pyruvate

$10.0 \mu \mathrm{M} \quad \beta$-Mercaptoethanol

$2.0 \mathrm{mM} \quad$ L-Glutamine 


$$
\begin{array}{ll}
20 \% & \text { Fetal calf serum (FCS) } \\
1000 \mathrm{U} / \mathrm{ml} & \text { Recombinant leukaemia inhibitory factor (LIF) }
\end{array}
$$

For cryopreservation of the cells in liquid nitrogen $8 \%$ DMSO have been added to the medium.

\section{$2.9 \quad$ Biologic material}

\subsubsection{Bacterial strains}

For the transformation of plasmids into competent bacterial cells, the Escherichia coli strain DH5 $\alpha$ (Hanahan 1983) was used and purchased from Life Technologies, Karlsruhe, Germany.

\subsubsection{Eukaryotic cell lines}

PC-3 Human prostate adenocarcinoma cell line (bone metastasis), ATCC, Rockville, USA, castration resistant cells (Kaighn et al. 1979; Ohnuki et al. 1980)

DU145 Human prostate adenocarcinoma cell lines (brain metastasis), ATCC, Rockville, USA, castration resistant cells (Stone et al. 1978)

LNCaP Human prostate adenocarcinoma cell line (lymphnode metastasis), ATCC, Rockville, USA, androgen dependent (Horoszewicz et al. 1983)

HeLa Human cervix adenocarcinoma cell line, ATCC, Rockville, USA

\subsubsection{Mouse strains}


All experiments were conducted according to the European and German protection of animals act. The number of sacrificed animals and the stress and pain they were suffering was kept to the minimum. Euthanasia of mice was performed either by $\mathrm{CO}_{2}$ asphyxation or cranial dislocation. Mice were kept at a 12 hours light/dark cycle at $22^{\circ} \mathrm{C}$ and $55 \pm 5 \%$ relative humidity. C57BL/ 6 wild type mice were obtained from a colony of our own department. EIIA-Cre mice (Dooley et al. 1989) were kindly provided by Nils Brose and Klaus-Armin Nave (Max Planck Institute of Experimental Medicine, Göttingen, Germany)

\subsubsection{Synthetic DNA-oligonucleotides}

For the generation of PCR products and for quantitative real time PCR analyses as well as for the EMSA experiments synthetic oligonucleotides were purchased from Eurofins MWG Operon (Ebersberg, Germany). Sequences are listed starting from 5'- to 3'- end.

Primer used for EMSA:

\begin{tabular}{l|l|l|l|l|l}
\hline Fragment & \multicolumn{2}{|l|}{ Sequence } \\
\hline \multirow{2}{*}{$\mathbf{8}$} & Fw & $5^{\prime}-$ & TTTT & TATGCCTGGTTCCTATTGGAAGCTCACAGGGGCTG & $-3^{\prime}$ \\
\cline { 2 - 7 } & Rev & $5^{\prime}-$ & TTTT & CAGCCCCTGTGAGCTTCCAATAGGAACCAGGCATA & $-3^{\prime}$ \\
\hline \multirow{2}{*}{$\mathbf{9}$} & Fw & $5^{\prime}-$ & TTTT & GGCTGACATCACTTAGGAAAGCGAAGGGGGTAGGG & $-3^{\prime}$ \\
\cline { 2 - 7 } & Rev & $5^{\prime}-$ & TTTT & CCCTACCCCCTTCGCTTTCCTAAGTGATGTCAGCC & $-3^{\prime}$ \\
\hline \multirow{2}{*}{$\mathbf{1 1}$} & Fw & $5^{\prime}-$ & TTTT & TAGGGCTGCCAGATCAGTTTGTCACCACCCAGGCT & $-3^{\prime}$ \\
\cline { 2 - 7 } & Rev & $5^{\prime}-$ & TTTT & AGCCTGGGTGGTGACAAACTGATCTGGCAGCCCTA & $-3^{\prime}$ \\
\hline \multirow{2}{*}{$\mathbf{1 2}$} & Fw & $5^{\prime}-$ & TTTT & AGGCTCCCTTGCCTTTGGCTGGGTGCAACTTCCAT & $-3^{\prime}$ \\
\cline { 2 - 7 } & Rev & $5^{\prime}-$ & TTTT & ATGGAAGTTGCACCCAGCCAAAGGCAAGGGAGCCT & $-3^{\prime}$ \\
\hline \multirow{2}{*}{$\mathbf{1 3}$} & Fw & $5^{\prime}-$ & TTTT & TCCATTTTAGGTGTTGGATCTGAGGGGGAAAAAAA & $-3^{\prime}$ \\
\cline { 2 - 7 } & Rev & $5^{\prime}-$ & TTTT & TTTTTTTCCCCCTCAGATCCAACACCTAAAATGGA & $-3^{\prime}$ \\
\hline \multirow{2}{*}{$\mathbf{1 4}$} & Fw & $5^{\prime}-$ & TTTT & AAAAAAGAGAGAGGGAGAGAGAGAGAAAGAAGAGC & $-3^{\prime}$ \\
\cline { 2 - 7 } & Rev & $5^{\prime}-$ & TTTT & GCTCTTCTTTCTCTCTCTCTCCCTCTCTCTTTTTT & $-3^{\prime}$ \\
\hline \multirow{2}{*}{$\mathbf{1 5}$} & Fw & $5^{\prime}-$ & TTTT & AGAGCAGGAAAGATCCCGAAAGGAGGAAGAGGTGG & $-3^{\prime}$ \\
\cline { 2 - 6 } & Rev & $5^{\prime}-$ & TTTT & CCACCTCTTCCTCCTTTCGGGATCTTTCCTGCTCT & $-3^{\prime}$ \\
\hline & Fw & $5^{\prime}-$ & TTTT & GGTGGCGAAAAATCAACTGCCCTGCTGGATTTGTC & $-3^{\prime}$ \\
\hline
\end{tabular}




\begin{tabular}{|c|c|c|c|c|c|}
\hline & Rev & $5^{\prime}-$ & TTTT & | GACAAATCCAGCAGGGCAGTTGATTTTTCGCCACC & -3 \\
\hline \multirow{2}{*}{16} & Fw & $5 '-$ & TTTT & TTGTCTTTCTCAGCACCTTGGCGAAGCCTTGGGTT & $-3^{\prime}$ \\
\hline & Rev & $5 '-$ & TTTT & AACCCAAGGCTTCGCCAAGGTGCTGAGAAAGACAA & $-3^{\prime}$ \\
\hline \multirow{2}{*}{17} & Fw & $5 '-$ & TTTT & GGGTTTCTTTCTTAAAGGACTGATTTTTAGAACTC & $-3^{\prime}$ \\
\hline & $\operatorname{Rev}$ & $5 '-$ & TTTT & GAGTTCTAAAAATCAGTCCTTTAAGAAAGAAACCC & $-3^{\prime}$ \\
\hline \multirow{2}{*}{18} & FW & $5 '-$ & TTTT & AACTCCACATTTGAGGTGTGTGGCTTTTGAAGAAA & $-3^{\prime}$ \\
\hline & Rev & 5 '- & TTTT & TTTCTTCAAAAGCCACACACCTCAAATGTGGAGTT & $-3^{\prime}$ \\
\hline \multirow{2}{*}{19} & FW & $5 '-$ & TTTT & AGAAAATGTATGTACTGACGGGAAAAGGAGGATAA & $-3^{\prime}$ \\
\hline & Rev & $5 '-$ & TTTT & TTATCCTCCTTTTCCCGTCAGTACATACATTTTCT & $-3^{\prime}$ \\
\hline \multirow{2}{*}{$1-2$} & FW & $5 '-$ & TTTT & TGTCTCCСTTGCCTCTTCCGCСCСССTCAGCTCT & $-3^{\prime}$ \\
\hline & Rev & 5 '- & TTTT & AGAGCTGAGGGGGGCGGAAGAGGCAAGGGAGACA & $-3^{\prime}$ \\
\hline \multirow[t]{2}{*}{$2-3$} & Fw & $5 '-$ & TTTT & CAGTAATTCCTCAGAGATTGTACAGTCTCTCCAC & $-3^{\prime}$ \\
\hline & Rev & $5 '-$ & TTTT & GTGGAGAGACTGTACAATCTCTGAGGAATTACTG & $-3^{\prime}$ \\
\hline \multirow[t]{2}{*}{ 3-4 } & Fw & 5 '- & TTTT & TTAAATATAAATATATAAATATAAATATATATAT & $-3^{\prime}$ \\
\hline & Rev & $5 '-$ & TTTT & ATATATATATTTATATTTATATATTTATATTTAA & $-3^{\prime}$ \\
\hline \multirow[t]{2}{*}{ 4-5 } & FW & 5 '- & TTTT & TATAAACTTCССССТGTCTTTCTCTССТСТСTTT & $-3^{\prime}$ \\
\hline & Rev & $5 '-$ & TTTT & AAAGAGAGGAGAGAAAGACAGGGGGAAGTTTATA & $-3^{\prime}$ \\
\hline \multirow[t]{2}{*}{$5-6$} & FW & $5 '-$ & TTTT & TTTTAATTTTCTATAATAAAGTTTCCTATTGGAT & $-3{ }^{\prime}$ \\
\hline & Rev & $5 '-$ & TTTT & ATCCAATAGGAAACTTTATTATAGAAAATTAAAA & $-3^{\prime}$ \\
\hline \multirow[t]{2}{*}{ 6-7 } & Fw & $5 '-$ & TTTT & AGGTCAGCTCCCTGATTTATGCCTGGTTCCTATTG & $-3{ }^{\prime}$ \\
\hline & Rev & $5 '-$ & TTTT & CAATAGGAACCAGGCATAAATCAGGGAGCTGACCT & $-3^{\prime}$ \\
\hline \multirow[t]{2}{*}{ 7-8 } & Fw & 5 '- & TTTT & AAGCTCACAGGGGCTGACATCACTTAGGAAAGCG & $-3^{\prime}$ \\
\hline & Rev & $5 '-$ & TTTT & CGCTTTCCTAAGTGATGTCAGCCCCTGTGAGCTT & -3 \\
\hline \multirow[t]{2}{*}{ 8-9 } & Fw & $5 '-$ & TTTT & AGGGGGTAGGGCTGCCAGATCAGTTTGTCACCAC & $-3^{\prime}$ \\
\hline & Rev & $5 '-$ & TTTT & GTGGTGACAAACTGATCTGGCAGCCCTACCCCCT & $-3^{\prime}$ \\
\hline \multirow[t]{2}{*}{ 9-10 } & Fw & 5 '- & TTTT & CAGGCTCCCTTGCCTTTGGCTGGGTGCAACTTCC & $-3^{\prime}$ \\
\hline & $\operatorname{Rev}$ & $5 '-$ & TTTT & GGAAGTTGCACCCAGCCAAAGGCAAGGGAGCCTG & $-3^{\prime}$ \\
\hline \multirow{2}{*}{$10-11$} & FW & $5 '-$ & TTTT & TTTTAGGTGTTGGATCTGAGGGGGAAAAAAAAGA & $-3^{\prime}$ \\
\hline & Rev & 5 '- & TTTT & TCTTTTTTTTСССССТСАGАТССААСАССТАААА & $-3^{\prime}$ \\
\hline \multirow{2}{*}{ 11-12 } & FW & $5 '-$ & TTTT & AGAGGGAGAGAGAGAGAAAGAAGAGCAGGAAAGA & $-3^{\prime}$ \\
\hline & Rev & 5 '- & TTTT & TCTTTCСTGCTCTTCTTTCTCTCTCTCTСССТСТ & $-3^{\prime}$ \\
\hline \multirow{2}{*}{$12-13$} & Fw & 5 '- & TTTT & CCCGAAAGGAGGAAGAGGTGGCGAAAAATCAACT & $-3^{\prime}$ \\
\hline & Rev & 5 '- & TTTT & AGTTGATTTTTCGCCACCTCTTCCTCCTTTCGGG & $-3^{\prime}$ \\
\hline
\end{tabular}




\begin{tabular}{l|l|l|l|l|l|l}
\multirow{2}{*}{$\mathbf{1 3 - 1 4}$} & Fw & $5^{\prime}-$ & TTTT & CCCTGCTGGATTTGTCTTTCTCAGCACCTTGGCG & $-3^{\prime}$ \\
\cline { 2 - 7 } & Rev & $5^{\prime}-$ & TTTT & CGCCAAGGTGCTGAGAAAGACAAATCCAGCAGGG & $-3^{\prime}$ \\
\hline \multirow{2}{*}{$\mathbf{1 4 - 1 5}$} & Fw & $5^{\prime}-$ & TTTT & AGCCTTGGGTTTCTTTCTTAAAGGACTGATTTTT & $-3^{\prime}$ \\
\cline { 2 - 7 } & Rev & $5^{\prime}-$ & TTTT & AAAAATCAGTCCTTTAAGAAAGAAACCCAAGGCT & $-3^{\prime}$ \\
\hline \multirow{2}{*}{$\mathbf{1 5 - 1 6}$} & Fw & $5^{\prime}-$ & TTTT & GAACTCCACATTTGAGGTGTGTGGCTTTTGAAGA & $-3^{\prime}$ \\
\cline { 2 - 7 } & Rev & $5^{\prime}-$ & TTTT & TCTTCAAAAGCCACACACCTCAAATGTGGAGTTC & $-3^{\prime}$ \\
\hline \multirow{2}{*}{$\mathbf{1 6 - 1 7}$} & Fw & $5^{\prime}-$ & TTTT & AATGTATGTACTGACGGGAAAAGGAGGATAAGCA & $-3^{\prime}$ \\
\cline { 2 - 7 } & Rev & $5^{\prime}-$ & TTTT & TGCTTATCCTCCTTTTCCCGTCAGTACATACATT & $-3^{\prime}$ \\
\hline
\end{tabular}

Vector-specific primer:

\begin{tabular}{l|l|l|l}
\hline \multicolumn{2}{l|}{ Primer name } & Sequence & \\
\hline Sp6new & $5^{\prime}-$ & TTAGGTGACACTATAGAATACTCAAGC & $-3^{\prime}$ \\
\hline T7new & $5^{\prime}-$ & AATACGACTCACTATAGGGCGAATTGG & $-3^{\prime}$ \\
\hline pGL3-fw & $5^{\prime}-$ & CTAGCAAAATAGGCTGTCCCCAGTGC & $-3^{\prime}$ \\
\hline pGL4-rev-129 & $5^{\prime}-$ & GCCCTTCTTAATGTTTTTGGCATCTTC & $-3^{\prime}$ \\
\hline
\end{tabular}

p120CTN-specific primer:

\begin{tabular}{l|l|l|l}
\hline \multicolumn{2}{l|}{ Primer name } & \multicolumn{2}{l}{ Sequence } \\
\hline p120Xho-240fw & $5^{\prime}-$ & CTCGAGTAAAAAGCCCTTGTTCCTTGTCTCCCTTGCC & $-3^{\prime}$ \\
\hline p120Xho-508fw & $5^{\prime}-$ & CTCGAGGCTCTAATGCAATTAATCCAAAAC & $-3^{\prime}$ \\
\hline p120Xho-1024fw & $5^{\prime}-$ & CTCGAGGGCATAAAATGTTCTTGACATGAGA & $-3^{\prime}$ \\
\hline p120Xho-1942fw2 & $5^{\prime}-$ & CTCGAGCCTACACTAGAGTGCAATGGCATG & $-3^{\prime}$ \\
\hline p120Bgl-TSrev & $5^{\prime}-$ & AGATCTCCTGTGAGCTTCCAATAGGAACCA & $-3^{\prime}$ \\
\hline p120Bgl+100rev & $5^{\prime}-$ & AGATCTCTAAAATGGAAGTTGCACCCAGCCAAAG & $-3^{\prime}$ \\
\hline p120Bgl+329rev & $5^{\prime}-$ & AGATCTGACAAAAATTCGACTTGCTTATCCT & $-3^{\prime}$ \\
\hline
\end{tabular}

Human-specific primer for quantitative real time PCR: 


\begin{tabular}{l|l|l|l}
\hline \multicolumn{2}{l|}{ Primer name } & & Sequence \\
\hline TBP-Fw & $5^{\prime}-$ & AGCCTGCCACCTTACGCTCAG & $-3^{\prime}$ \\
\hline TBP-Rev & $5^{\prime}-$ & TGCTGCCTTTGTTGCTCTTCCA & $-3^{\prime}$ \\
\hline PBGD-Fw & $5^{\prime}-$ & GCAATGCGGCTGCAACGGCGGAAG & $-3^{\prime}$ \\
\hline PBGD-Rev & $5^{\prime}-$ & CCTGTGGTGGACATAGCAATGATT & $-3^{\prime}$ \\
\hline LPXN-CDS-F1 & $5^{\prime}-$ & CCCAGAGCACTTCTTCTGCT & $-3^{\prime}$ \\
\hline LPXN-CDS-R1 & $5^{\prime}-$ & GCTAAGAAATCCTTTCGGCA & $-3^{\prime}$ \\
\hline ITGB1-Q2-Fw & $5^{\prime}-$ & CCCATTGTAAGGAGAAGGATGTTG & $-3^{\prime}$ \\
\hline ITGB1-Q2-Rev & $5^{\prime}-$ & CAAGGCCAATAAGAACAATTCCAG & $-3^{\prime}$ \\
\hline
\end{tabular}

Mouse-specific primer for genotyping:

\begin{tabular}{l|c|l|c}
\hline \multicolumn{1}{c|}{ Primer name } & \multicolumn{2}{c}{ Sequence } & $-3^{\prime}$ \\
\hline LPXN-5' & $5^{\prime}-$ & CTCAGGCTGTAATGGGTATGAAG & $-3^{\prime}$ \\
\hline LPXN-3' & $5^{\prime}-$ & GCTATGGCTTTACAGCTCTGTTCT & $-3^{\prime}$ \\
\hline LPXN-Fw & $5^{\prime}-$ & AGTTGGATGAGCTCATGGCCCACC & $-3^{\prime}$ \\
\hline LoxR & $5^{\prime}-$ & TGAACTGATGGCGAGCTCAGACC & $-3^{\prime}$ \\
\hline mLPXN-In-3-4-Fw & $5^{\prime}-$ & AGTTCTCCTTACTTTTGCCAGCA & $-3^{\prime}$ \\
\hline mLPXN-In-4-5-Rev & $5^{\prime}-$ & CGGCGTGATCATGCAGAA & \\
\hline
\end{tabular}

\subsubsection{Synthetic RNA oligonucleotides}

The used small interfering RNAs were purchased from Life Technologies (Darmstadt, Germany).

Human LPXN “siLPXN"

Target sequence:

5'-GGGGCAGCUCGUGUAUACUACCAAU-3'

Sense strand:

5'-GGGGCAGCUCGUGUAUACUACCAAUdTdT-3'

Anti-sense strand:

5'-AUUGGUAGUAUACACGAGCUGCGCCdTdT-3' 
Luciferase (Photinuspyralis) "siLuc"

Target sequence: 5'-CGUACGCGGAAUACUUCGA-3'

Sense strand:

5'-CGUACGCGGAAUACUUCGAdTdT-3'

Anti-sense strand:

5'-UCGAAGUAUUCCGCGUACGdTdT-3'

\subsubsection{Antibodies}

\subsubsection{Inhibitory antibodies}

\begin{tabular}{l|l}
\hline Antibody & Source \\
\hline$\beta 1$-integrin inhibitory antibody, clone & Kindly provided by Prof. Dr. Nils Cordes, \\
AIIB2, monoclonal antibody, rat & OncoRay, Dresden, Germany \\
\hline Rat IgG - Isotype Control (ab37361) & Abcam, Cambridge, UK \\
\hline
\end{tabular}

\subsubsection{Primary antibodies}

\begin{tabular}{l|l}
\hline Primary antibody & Manufacturer \\
\hline $\begin{array}{l}\text { Anti-LPXN (283 G), monoclonal } \\
\text { antibody, mouse }\end{array}$ & ICOS Corp., Bothell, USA \\
\hline $\begin{array}{l}\text { Anti-Caldesmon (8-L-Caldesmon), } \\
\text { monoclonal antibody, mouse } \\
\text { (\#610660) }\end{array}$ & BD Transduction Laboratories, \\
\hline Anti-Integrin $\beta 1$ (D2E5) monoclonal \\
antibody, rabbit (\#9699) & Cell Signaling, Danvers, MA, USA \\
\hline $\begin{array}{l}\text { Anti-phospho-Caldesmon Ser-789, } \\
\text { polyclonal antibody, rabbit (sc-12931) }\end{array}$ & Santa Cruz Biotechnology, Heidelberg, \\
\hline $\begin{array}{l}\text { Anti-p44/42 MAPK (ERK1/2), } \\
\text { polyclonal antibody, rabbit }\end{array}$ & Cell Signaling, Danvers, MA, USA \\
\hline $\begin{array}{l}\text { Anti-phospho-p44/42 MAPK } \\
\text { (ERK1/2) (Thr202/Tyr204) D13.13E4 }\end{array}$ & Cell Signaling, Danvers, MA, USA \\
\hline
\end{tabular}




\begin{tabular}{l|l}
\hline $\begin{array}{l}\text { Anti-STAT1 } \alpha \text { p91 (C-24), polyclonal } \\
\text { antibody, rabbit }\end{array}$ & $\begin{array}{l}\text { Santa Cruz Biotechnology, Heidelberg, } \\
\text { Germany }\end{array}$ \\
\hline $\begin{array}{l}\text { Anti- } \alpha \text {-Tubulin (clone B-5-1-2), } \\
\text { monoclonal antibody, mouse }\end{array}$ & Sigma-Aldrich, Deisenhofen, Germany \\
\hline $\begin{array}{l}\text { Anti-Leupaxin (N-terminal), polyclonal } \\
\text { antibody, rabbit (SAB4200010) }\end{array}$ & Sigma-Aldrich, Deisenhofen, Germany \\
\hline $\begin{array}{l}\text { Anti-cmyc Tag (clone 4A6), } \\
\text { monoclonal antibody, mouse }\end{array}$ & Sigma-Aldrich, Deisenhofen, Germany
\end{tabular}

\subsubsection{Secondary antibodies}

\begin{tabular}{l|l}
\hline Secondary antibody & Manufacturer \\
\hline $\begin{array}{l}\text { Anti-rabbit IgG, alkaline phosphatase } \\
\text { conjugated (A-3687), goat }\end{array}$ & Sigma-Aldrich, Deisenhofen, Germany \\
\hline $\begin{array}{l}\text { Anti-rabbit IgG (H+L), HRP (horse } \\
\text { radish peroxidase) conjugated, goat }\end{array}$ & $\begin{array}{l}\text { Dianova Jackson ImmunoResearch, } \\
\text { Hamburg, Germany }\end{array}$ \\
\hline $\begin{array}{l}\text { Anti-rabbit IgG, Cy3-conjugated } \\
\text { (C2306) }\end{array}$ & Sigma-Aldrich, Deisenhofen, Germany \\
\hline Anti-mouse IgG, alkaline phosphatase- & Sigma-Aldrich, Deisenhofen, Germany \\
\hline Anjiugated (A-3688), rabbit & Dianova Jackson ImmunoResearch, \\
conjugated, rabbit & Hamburg, Germany \\
\hline $\begin{array}{l}\text { Anti-mouse IgG, Cy3-conjugated } \\
\text { (C2181) }\end{array}$ & Sigma-Aldrich, Deisenhofen, Germany \\
\hline
\end{tabular}

\subsubsection{Plasmids and Vectors}

\begin{tabular}{l|l}
\hline \multicolumn{1}{c|}{ Plasmid/Vector } & \multicolumn{1}{c}{ Source } \\
\hline pGEM-Teasy & Promega, Wisconsin, USA \\
\hline pGL4.16 luc2CP/Hygro & Promega, Wisconsin, USA \\
\hline pGL4.50 luc2CP/CMV/Hygro & Promega, Wisconsin, USA \\
\hline pCAGGS-FLPe & kindly provided by Prof. Dr. Ibrahim \\
\hline
\end{tabular}




\begin{tabular}{l|l} 
& Adham \\
\hline pEGFP-LPXN & Dr. S. Kaulfuß, 2006 \\
\hline pEGFP-LPXN-NLS & Sascha Dierks, 2011 \\
\hline pBACe3.6-CTNND1 & $\begin{array}{l}\text { Clontech-Takara, Saint-Germain-en-Laye, } \\
\text { France }\end{array}$ \\
(RZPDB737G032171D) & ImaGenes GmbH, Berlin, Germany \\
\hline pCMV-Myc & Clontech-Takara, Saint-Germain-en-Laye, \\
\hline pCMV-Myc-LPXN & France \\
\hline pCMV-Myc-Caldesmon & Dr. S. v.Hardenberg, 2010 \\
\hline pRL-Luc & Dr. S. v.Hardenberg, 2010 \\
\hline
\end{tabular}

\subsubsection{Used constructs and plasmids}

\begin{tabular}{l|l|l|l}
\hline \multicolumn{1}{c|}{ Construct } & Plasmid/Vector & \multicolumn{1}{c}{ Source } & Primer/ restriciton site \\
\hline pGL4.16-p120CTN- & pGL4.16 & pBACe.3.6 & P120Xho-240fw / p120Bgl- \\
200-TS & luc2CP/Hygro & -CTNND1 & TSrev \\
\hline pGL4.16-p120CTN- & pGL4.16 & pBACe.3.6 & P120Xho-240fw / \\
200-100 & luc2CP/Hygro & -CTNND1 & p120Bgl+100rev \\
\hline pGL4.16-p120CTN- & pGL4.16 & pBACe.3.6 & P120Xho-240fw / \\
200-300 & luc2CP/Hygro & -CTNND1 & p120Bgl+329rev \\
\hline pGL4.16-p120CTN- & pGL4.16 & pBACe.3.6 & P120Xho-508fw / p120Bgl- \\
$500-T S$ & luc2CP/Hygro & -CTNND1 & TSrev \\
\hline pGL4.16-p120CTN- & pGL4.16 & pBACe.3.6 & P120Xho-508fw / \\
500-100 & luc2CP/Hygro & -CTNND1 & p120Bgl+100rev \\
\hline pGL4.16-p120CTN- & pGL4.16 & pBACe.3.6 & P120Xho-508fw / \\
$500-300$ & luc2CP/Hygro & -CTNND1 & p120Bgl+329rev \\
\hline pGL4.16-p120CTN- & pGL4.16 & pBACe.3.6 & P120Xho-1048fw / \\
$1 \mathrm{kb-TS}$ & luc2CP/Hygro & -CTNND1 & p120Bgl+TSrev \\
\hline pGL4.16-p120CTN- & pGL4.16 & pBACe.3.6 & P120Xho-1048fw / \\
1kb-100 & luc2CP/Hygro & -CTNND1 & p120Bgl+100rev \\
\hline pGL4.16-p120CTN- & pGL4.16 & pBACe.3.6 & P120Xho-1048fw / \\
\hline
\end{tabular}




\begin{tabular}{l|l|l|l}
$1 \mathrm{~kb}-300$ & luc2CP/Hygro & -CTNND1 & p120Bgl+329rev \\
\hline pGL4.16-p120CTN- & pGL4.16 & pBACe.3.6 & P120Xho-1942fw2 / \\
2kb-300 & luc2CP/Hygro & -CTNND1 & p120Bgl+300rev
\end{tabular}

\subsection{Databases}

\begin{tabular}{l|l}
\hline \multicolumn{1}{c|}{ Usage } & \multicolumn{1}{c}{ Programm } \\
\hline \multirow{2}{*}{ Restriction site analysis } & NEB Cutter 2.0 \\
& (http://tools.neb.com/NEBcutter2/index. \\
& php) \\
\cline { 3 - 3 } & WEB Cutter 2.0 \\
& (htp://rna.lundberg.gu.se/cutter2) \\
\hline Analysis of DNA- and protein & BLAST-program (Altschulet al. 1990) \\
sequences & (http://ncbi.nlm.nih.gov) \\
\hline \multirow{2}{*}{ Bioinformatics } & Ensembl v32 (http://www.ensembl.org) \\
\cline { 3 - 3 } & Nation Center for Biotechnology \\
& Information (http://ncbi.nlm.nih.goc) \\
\hline \multirow{2}{*}{ Primer design } & Primer3 (http://frofo.wi.mit.edu/chi- \\
& bin/primer3/primer3_www.cgi)
\end{tabular}

\subsection{Isolation and purification of nucleic acids}

\subsubsection{Minipreparation of plasmid DNA}

For rapid isolation of recombinant plasmids, a small amount of plasmid DNA was prepared. Therefore, $1 \mathrm{ml}$ of a bacterial overnight-culture was centrifuged at $5000 \mathrm{x} \mathrm{g}$ and the pellet was resuspended in $250 \mu \mathrm{l}$ P1 solution. After adding $250 \mu \mathrm{l}$ of P2 (modified alkaline lysis) to the suspension, it was incubated at RT for $5 \mathrm{~min}$. Next, $250 \mu \mathrm{l}$ of P3 (neutralization) was pipetted to the sample and centrifuged at $13.000 \mathrm{x}$. The supernatant was transferred into a reaction tube containing $500 \mu \mathrm{l}$ of ice cold $0.7 \mathrm{vol}$ isopropanol. The plasmid DNA was precipitated and centrifuged at $16.000 \mathrm{xg}$ for $45 \mathrm{~min}$. Afterwards, the DNA pellet was washed with $200 \mu \mathrm{l}$ of $70 \%$ ethanol. Finally, the DNA was dried and resolved in $20-30 \mu \mathrm{ldd} \mathrm{H}_{2} \mathrm{O}$. 


\subsubsection{Establishment of bacterial glycerol stocks}

$100 \mu \mathrm{l}$ sterile glycerol was added to $1 \mathrm{ml}$ of a bacterial suspension, well mixed and stored at $-80^{\circ} \mathrm{C}$.

\subsubsection{Midipreparation of plasmid DNA}

For isolation of larger amounts of ultra-pure plasmid DNA, the PureLink ${ }^{\mathrm{TM}}$ HiPure Plasmid Midiprep Kit from Life Technologies (Darmstadt, Germany) was used. This kit uses affinity chromatography columns to purify the plasmid DNA. The purification was performed according to the manufacturer's instructions. DNA purified by this method can be used for the transfection of cell lines, restriction analysis, subcloning or sequencing.

\subsubsection{Isolation of total RNA from cell cultures}

For isolation of total RNA from cell cultures the pegGOLD Total RNA Kit (Peqlab, Erlangen, Germany) was used according to the manufactures instructions.

\subsubsection{Determination of nucleic acid concentration}

The concentration of nucleic acids was determined by a spectral photometer (BioPhotometer, Eppendorf, Hamburg). After the blank value was set, the absorption maximum of the nucleic acids $(260 \mathrm{~nm})$ was measured. Simultaneously, contaminations by proteins $(280 \mathrm{~nm})$ or salts $(320 \mathrm{~nm})$ were detected. The concentration of nucleic acids was calculated according to the Lambert's law.

Lower concentrations were determined by agarose gel electrophoresis. A certain amount of DNA was loaded onto an agarose gel and run with a DNA standard (MassRuler DNA Ladder, Thermo Scientific, Langenselbold, Germany), for which the amount of DNA for each band was known. After the gel was run, a photograph of the stained DNA was taken and the concentration of the sample was estimated from the band intensities. 


\subsection{Cloning techniques}

\subsubsection{Cleavage of DNA with restriction endonucleases}

For enzymatic cleavage of DNA by restriction endonucleases, a restriction reaction in a total volume of at least $10 \mu \mathrm{l}$ was prepared. Per $\mu \mathrm{g}$ DNA $1 \mathrm{U}$ of the respective restriction enzyme was used. Together with the corresponding buffer the reaction was incubated at $37{ }^{\circ} \mathrm{C}$ for $1-2$ hours or overnight. In case the DNA was cleaved by two restriction endonucleases, the buffer was chosen for sufficient activity of both enzymes or a sequential restriction was performed.

\subsubsection{Isolation of DNA fragments from agarose gels}

For isolation of DNA fragments from agarose gels, the NucleoSpin ${ }^{\mathrm{TM}}$ Extract II Kit (Macherey \& Nagel, Düren, Germany) was used. After the DNA fragments were separated by gel electrophoresis on an agarose gel, they were cut out of the gel using a sterile scalpel and dissolved in the corresponding buffer at $50^{\circ} \mathrm{C}$. Subsequently, the DNAagarose solution was loaded onto the columns and purified according to the manufacturer's instructions. The DNA was eluted in $\mathrm{ddH}_{2} \mathrm{O}$.

\subsubsection{Dephosphorylation of plasmid DNA}

To avoid religation of linearized plasmid DNA, the terminal 5'-phosphate group of the vector was removed by alkaline phosphatase. Therefore, the DNA was incubated with 2 $\mathrm{U}$ alkaline phosphatase for 1 hour at $37^{\circ} \mathrm{C}$. After dephosphorylation the DNA was purified using silica membrane columns (Invitek, Berlin, Germany) and resuspended in $\mathrm{ddH}_{2} \mathrm{O}$.

\subsubsection{Ligation of DNA fragments}


In a ligation reaction, a phosphodiester bond is formed between the 3'-hydroxy- and 5'phosphate group of the linearized DNA. This enzymatic reaction is catalyzed by the T4 DNA ligase leading to the generation of recombinant DNA molecules.

The following reaction mixture was used:

$$
\begin{array}{ll}
25-50 \mathrm{ng} & \text { vector DNA (digested) } \\
30-120 \mathrm{ng} & \text { insert DNA } \\
1 \mu \mathrm{l} & \text { T4 DNA ligase }(5 \mathrm{U} / \mu \mathrm{l}) \\
1 \mu \mathrm{l} & \text { ligation buffer }(10 \mathrm{x}) \\
\text { ad } 10 \mu \mathrm{H}_{2} \mathrm{O} &
\end{array}
$$

The ligation reaction was carried out at RT for 2 hours or at $4^{\circ} \mathrm{C}$ overnight.

\subsubsection{Subcloning of PCR and RT PCR products / TA cloning}

(Clark 1988); (Hu 1993)

Taq- and other polymerases possess a terminal transferase activity that results in the non-template addition of a single nucleotide to the 3 '-ends of PCR products. This terminal transferase activity is the basis of the TA cloning strategy. For cloning of PCR products, the pGEM-Teasy vector system that has $5^{\prime}$-dT overhangs was used. For products that were amplified with Phusion ${ }^{\mathrm{TM}}$ High-Fidelity DNA polymerase (Finnzymes, Finland), which does not carry such a terminal transferase activity, the terminal dA had to be added before the PCR product could be cloned into the pGEM-Teasy vector. Therefore, the following A-tailing reaction was conducted:

$$
\begin{array}{ll}
7 \mu \mathrm{l} & \text { PCR product } \\
0.7 \mu \mathrm{l} & \text { MangoTaq DNA polymerase }(1 \mathrm{U} / \mu \mathrm{l}) \\
0.3 \mu \mathrm{l} & \mathrm{MgCl}_{2}(50 \mathrm{nM}) \\
1 \mu \mathrm{l} & \mathrm{dATP}(2 \mathrm{mM}) \\
1 \mu \mathrm{l} & 10 \text { x polymerase buffer }
\end{array}
$$

The reaction mixture was incubated for $30 \mathrm{~min}$ at $70^{\circ} \mathrm{C}$. 
For cloning into the pGEM-Teasy vector $1-2 \mu \mathrm{l}$ of the A-tailing mixture were used in a ligation reaction.

\author{
50 ng pGEM-Teasy \\ PCR product (3:1, insert : vector ratio) \\ $1 \mu \mathrm{l}$ T4 DNA ligase $10 \mathrm{x}$ buffer \\ $1 \mu \mathrm{l}$ T4 DNA ligase \\ ad $10 \mu \mathrm{l} \mathrm{H}_{2} \mathrm{O}$
}

\title{
2.12.6 Transformation of competent bacteria with plasmid DNA
}

(Hanahan 1983)

For the transformation of competent bacteria a $50 \mu \mathrm{l}$ aliquot was gently mixed with 3 $5 \mu \mathrm{l}$ of the ligation reaction. After incubation for $20 \mathrm{~min}$ on ice, a heat shock was performed for $1 \mathrm{~min}$ at $42^{\circ} \mathrm{C}$. After cooled down on ice for $5 \mathrm{~min} 200 \mu \mathrm{l}$ of LB medium was added, before the bacteria were incubated on a thermo shaker at $37^{\circ} \mathrm{C}$ for $45 \mathrm{~min}$. Finally, $50-200 \mu \mathrm{l}$ of the transformed bacteria were plated out on LB agar plates containing appropriate antibiotics and incubated at $37^{\circ} \mathrm{C}$ overnight.

\section{$2.13 \quad$ Gel electrophoresis}

Gel electrophoresis is a technique that uses an electric field to separate charged macromolecules, especially nucleic acids and proteins. The resolution of the separation is dependent on the density (percentage) of the gel as well as on the charge to mass ratio of the corresponding macromolecule.

\subsubsection{Agarose gel electrophoresis of DNA}

Agarose gels are used to separate nucleic acid molecules. Depending on the fragment size gels with an agarose concentration of $0.5-2.0 \%(\mathrm{w} / \mathrm{v})$ were prepared. According to the gel size the agarose was resolved by boiling in 30 or $60 \mathrm{ml}$ of 1x Turbo buffer. DNA Stain G (Serva GmbH, Heidelberg, Germany) was added according to the 
manufacturer's instructions, before the agarose gel was poured into a horizontal gel chamber. After the gel was completely solidified, gel electrophoresis was performed in $1 \mathrm{x}$ Turbo buffer at a constant voltage between $50-200 \mathrm{~V}$.

\subsubsection{Length standard}

For the determination of DNA fragment length on agarose gels, length standards were loaded in parallel.

$1 \mathrm{~Kb}$ Plus DNA-ladder

MassRuler ${ }^{\circledR}$
Life Technologies, Darmstadt, Germany

MBI Fermentas, St. Leon-Rot, Germany

\subsection{Polymerase Chain Reaction (PCR)}

(Saiki et al. 1992)

The polymerase chain reaction is an in vitro method to amplify specific DNA fragments. This method uses high temperatures to denature double stranded DNA and to allow short oligonucleotides (primers) to bind to DNA (annealing). Once bound to single stranded DNA these oligonucleotides are extended by the polymerase (elongation). These steps of denaturation, annealing and elongation are repeated periodically leading to an exponential increase in DNA fragments. Successful amplification can be checked by agarose gel electrophoresis.

\subsubsection{Amplification of DNA fragments from plasmid DNA}

For amplification, a specific pair of primers defined the DNA-region of interest. For the amplification of DNA fragments designated for cloning, the Phusion ${ }^{\text {TM }}$ High-Fidelity DNA polymerase (Finnzymes, Finland), which in addition to its $5^{\prime} \rightarrow 3^{\prime}$ DNA polymerase activity also carries a 3' $\rightarrow$ 5' exonuclease (proofreading) activity, was used. PCR reaction mixture was the following: 
Final conc.

\begin{tabular}{|c|c|c|}
\hline $\mathrm{X} \mu \mathrm{l}$ & template DNA $(10-50 \mathrm{ng})$ & \\
\hline $1 \mu \mathrm{l}$ & Primer 1 (10pmol/ $\mu$ l, sequence-specific) & $0.5 \mu \mathrm{M}$ \\
\hline $1 \mu \mathrm{l}$ & Primer $2(10 \mathrm{pmol} / \mu \mathrm{l}$, sequence-specific) & $0.5 \mu \mathrm{M}$ \\
\hline $0.4 \mu \mathrm{l}$ & 10 mMdNTPs & $200 \mu \mathrm{M}$ each \\
\hline $4 \mu \mathrm{l}$ & Phusion $^{\mathrm{TM}}$ HF Buffer ( $5 \mathrm{x}$ ) & $1 \mathrm{x}$ \\
\hline $\begin{array}{l}0.2 \mu \mathrm{l} \\
\operatorname{ad} 20 \mu \mathrm{l}\end{array}$ & $\begin{array}{l}\text { Phusion }^{\mathrm{TM}} \text { High-Fidelity DNA Polymerase } \\
\mathrm{ddH}_{2} \mathrm{O}\end{array}$ & $0.02 \mathrm{U} / \mu \mathrm{l}$ \\
\hline
\end{tabular}

25 to 35 amplification cycles were carried out in a Primus 25 advanced Thermocycler from Peqlab (Erlangen, Germany) or in a 2720 Thermal Cycler from Applied Biosystems (California, USA), respectively. The temperatures, the length of the corresponding PCR steps and the number of cycles were accordingly adjusted for every DNA fragment. One PCR cycle consisted of:

\begin{tabular}{|c|c|c|c|}
\hline Cycle step & Temp. & Time & Cycles \\
\hline Initial denaturation & $98^{\circ} \mathrm{C}$ & $30 \mathrm{sec}$ & 1 \\
\hline Denaturation & $98^{\circ} \mathrm{C}$ & $5-10 \mathrm{sec}$ & \\
\hline Annealing & $\mathrm{X}^{\circ} \mathrm{C}$ & $10-30 \mathrm{sec}$ & $25-35$ \\
\hline Elongation & $72^{\circ} \mathrm{C}$ & $15-30 \mathrm{sec} / 1 \mathrm{~Kb}$ & \\
\hline Final elongation & $72^{\circ} \mathrm{C}$ & $5-10 \min$ & \\
\hline
\end{tabular}

\subsubsection{Reverse Transcription}


The reverse transcription (RT)-PCR is a method that makes use of a reverse transcriptase to transcribe RNA into cDNA. The principle of reverse transcription relies on the application of an oligo(dT)-primer, which anneals to the poly(A)-sequences of mRNAs. Analog to the standard PCR the reverse transcriptase can elongate the strand to synthetize cDNA. To generate cDNA from total RNA the SuperScript II (Life Technologies, Darmstadt, Germany) was used. Subsequently, the reverse transcribed cDNA was subjected to quantitative real time PCR analyses.

The following reaction was performed in three steps:

1.Step:

$$
\begin{aligned}
& 2 \text { - } 5 \mu \mathrm{g} \text { RNA } \\
& +0.5 \mu \mathrm{l} \text { oligo(dT)-primer }(0.5 \mu \mathrm{g} / \mu \mathrm{l}) \\
& +0.5 \mu \mathrm{dNTP}(10 \mathrm{mM}) \\
& \text { ad } 6 \mu \mathrm{ldd} \mathrm{H}_{2} \mathrm{O} \\
& \text { Incubation: } 72^{\circ} \mathrm{C} \text { for } 10 \mathrm{~min}
\end{aligned}
$$

2. Step:

$+2 \mu \mathrm{l} 5 \mathrm{x}$ First Strand Buffer

$$
+1 \mu \mathrm{DTT}(0.1 \mathrm{M})
$$

Incubation: $42^{\circ} \mathrm{C}$ for $2 \mathrm{~min}$

3. Step:

$$
\begin{aligned}
& +0.25 \mu \mathrm{l} \text { SuperScript II } \\
& +0.75 \mu \mathrm{ldd} \mathrm{H}_{2} \mathrm{O}
\end{aligned}
$$

Reverse Transcription: $42^{\circ} \mathrm{C}$ for $50 \mathrm{~min}$

Heat-inactivation: $70^{\circ} \mathrm{C}$ for $15 \mathrm{~min}$

Store at $8^{\circ} \mathrm{C}$ for $\infty$

The synthetized cDNA was checked by PCR using specific primers for the housekeepinggene Glyceralaldehyde 3-phosphate dyhydrogenase (GAPDH), before it was subjected to quantitative real time PCR analyses.

\subsubsection{Quantitative real time PCR-analyses}


The quantitative real time PCR is a method that relies on the basic PCR principle but allows the quantification of the PCR product during the PCR. To quantify the DNA a fluorescent dye, which is binding unspecifically to double stranded DNA is applied to the mixture. In the present study the PCR Mastermix Platinum ${ }^{\circledR}$ SYBR $^{\circledR}$ Green qPCRSuperMix-UDG with Rox (Life Technologies, Darmstadt, Germany) was used. The rox dye serves as reference to correct for pipetting errors. SYBR ${ }^{\circledR}$ Green, which is bound to double stranded DNA can be excited using light of $480 \mathrm{~nm}$ wavelength and shows an emission spectrum with a maximum at $520 \mathrm{~nm}$. The fluorescent intensity was measured after every PCR cycle using the ABI Prism 7900T Sequence Detection System and a graph was generated. During the exponential phase of the PCR reaction conditions are optimal, meaning that maximum polymerase activity is attained and enough reaction materials (Primer, $\mathrm{MgCl}_{2}$ ) are present. During this phase the threshold value is determined, which defines the PCR cycle in which these conditions are met. This value is used for further calculations for quantification (Ct value). These data were evaluated with the Sequence-detection system software (SDS Version 2.1, PE Applied Biosystems). Prior to quantitative real time PCR the cDNA was usually diluted 1:20 in $\mathrm{ddH}_{2} \mathrm{O}$.

The reaction was comprised of the following components:

$$
\begin{aligned}
& 2.5 \mu \mathrm{l} \mathrm{cDNA} \\
& 2.5 \mu \mathrm{l} \text { Primer }(\mathrm{Fw}+\operatorname{Rev} 100 \mathrm{pmol} / \mu \mathrm{l}) \\
& 5.0 \mu \mathrm{l} \mathrm{SYBR}{ }^{\circledR} \text { Green }
\end{aligned}
$$

The program on the ABI Prism 7900T Sequence Detection System was the following:

$\begin{array}{lll}50^{\circ} \mathrm{C} & 2 \mathrm{~min} & \\ 95^{\circ} \mathrm{C} & 3 \mathrm{~min} & \text { Taq activation } \\ 94^{\circ} \mathrm{C} & 15 \mathrm{sec} & \text { Denaturation } \\ 60^{\circ} \mathrm{C} & 30 \mathrm{sec} & \text { Annealing } \\ 72^{\circ} \mathrm{C} & 30 \mathrm{sec} & \text { Elongation } \\ 95^{\circ} \mathrm{C} & 15 \mathrm{sec} & \\ 60^{\circ} \mathrm{C} & 15 \mathrm{sec} & \\ 60^{\circ} \mathrm{C}-95^{\circ} \mathrm{C} & 2^{\circ} \mathrm{C} / \mathrm{min} & \text { Melting curve }\end{array}$


After measurement the data was transferred to MS Excel (Microsoft) for further calculations. Relative expression was determined by the $\Delta \Delta$-Ct-method, which uses the following formula:

$$
\begin{aligned}
& \Delta \mathrm{Ct}=\mathrm{Ct} \text { (Gene of interest) }-\mathrm{Ct} \text { (housekeeping gene) } \\
& \Delta \Delta \mathrm{Ct}=\Delta \mathrm{Ct} \text { (Control) }-\Delta \mathrm{Ct} \text { (Sample of interest) } \\
& \text { Relative expression }=2^{\Delta \Delta \mathrm{Ct}}
\end{aligned}
$$

The mRNA expression of the housekeeping genes TATA-binding protein (TBP) and Porphobilinogendeaminase (PBGD) was used as a reference.

\subsubsection{Sequence analysis / DNA-sequencing after Sanger}

The non-radioactive sequencing is based on the principle of chain termination developed by (Sanger, Coulson 1975). This method uses four dideoxynucleotides that have been labeled with different fluorescent dyes. These dideoxynucleotides do not possess a 3'-hydroxy group. In case of incorporation of such a dideoxynucleotide into the DNA, this leads to a chain termination. The sequence reaction was performed under following conditions:

$\begin{array}{ll}1 \mu \mathrm{l} & \text { DNA } \\ 2 \mu \mathrm{l} & 5 x \text { Buffer } \\ 1 \mu \mathrm{l} & \text { Sequence-specific Primer }(10 \mathrm{pmol} / \mu \mathrm{l}) \\ 1 \mu \mathrm{l} & \text { BigDye }^{\circledR} \\ 5 \mu \mathrm{l} & \mathrm{ddH}_{2} \mathrm{O}\end{array}$

Sequencing program:

$\begin{array}{lll}\text { Initial denaturation } & 95^{\circ} \mathrm{C} & 1 \mathrm{~min} \\ {\left[\begin{array}{lll}\text { Denaturation } & 95^{\circ} \mathrm{C} & 30 \mathrm{sec} \\ \text { Annealing } & 60^{\circ} \mathrm{C} & 2.5 \mathrm{~min}\end{array}\right] 30 \text { cycles }}\end{array}$




\begin{tabular}{|c|c|}
\hline Final elongation & $60^{\circ} \mathrm{C}$ \\
\hline & $8^{\circ} \mathrm{C}$ \\
\hline
\end{tabular}

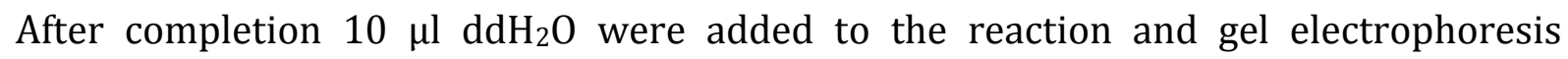
through specific capillaries was performed in the automatic sequencer 3500XL (Applied Biosystems, Life Technologies, Darmstadt, Germany).

\subsection{Protein chemical techniques}

\subsubsection{Isolation of total protein from cell cultures}

For isolation of total protein from cell cultures cultured in 6-well plates or T-25 culture flasks, the medium was removed and the cells were washed with ice cold PBS. After approx. $150 \mu$ of lysis buffer for protein (modified RIPA) were added the adherent cells were scraped off the bottom using a cell scraper or pipet tip and incubated for $5 \mathrm{~min}$ on ice. The amount of lysis buffer was adjusted to the confluence and size of the culture vessel. Next, the lysate was transferred to a microcentrifuge tube and centrifuged at $16.000 \mathrm{rpm}$ at $4^{\circ} \mathrm{C}$ for $10 \mathrm{~min}$ to pellet cell debris. The supernatant was transferred to a new microcentrifuge tube and stored at $-20^{\circ} \mathrm{C}$ after the protein concentration was determined. For long-term storage the lysates were kept at $-80^{\circ} \mathrm{C}$.

\subsubsection{Isolation of nuclear extracts}

PCa cells were grown on 6-well plates and lysed in $50 \mu \mathrm{l}$ cytoplasmic extraction buffer for $5 \mathrm{~min}$ on ice. After centrifugation at $13.000 \mathrm{rpm}$ for $15 \mathrm{sec}$ at $4^{\circ} \mathrm{C}$, the supernatants were spun again for $5 \mathrm{~min}$ at $13.000 \mathrm{rpm}$ and $4^{\circ} \mathrm{C}$ and the supernatant was collected as cytoplasmic extract in a new Microcentrifuge tube. The pellet from the first centrifugation was lysed in $50 \mu \mathrm{l}$ nuclear extraction buffer for $30 \mathrm{~min}$ on ice. After centrifugation at $13.000 \mathrm{rpm}$ and $4^{\circ} \mathrm{C}$ for $15 \mathrm{~min}$, the supernatant was collected as nuclear extract and either directly used for EMSA or stored at $-80^{\circ} \mathrm{C}$. 


\subsubsection{Determination of protein concentration}

(Bradford 1976)

Determination of protein concentration was performed using the Roti ${ }^{\circledR}$-Nanoquant (Carl Roth GmbH, Karlsruhe, Germany) according to the method of Bradford. This method is suitable for a protein amount ranging from $200 \mathrm{ng}$ to $25 \mu \mathrm{g}$ and uses the Coomassie Brilliant Blue dye. In acidic solution this dye binds unspecifically to cationic and hydrophobic site chains of proteins. Upon binding the absorption maximum of this dyeis shifted from $495 \mathrm{~nm}$ to $595 \mathrm{~nm}$. From measurement of a standard protein concentration (bovine serum albumine) a straight calibration line is compiled.

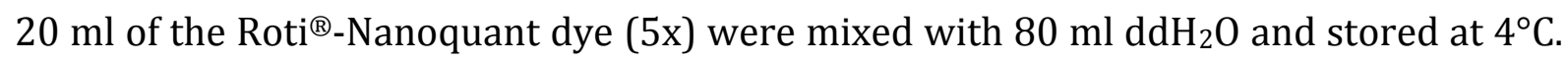
Proteins were diluted 1:100 and triplicates of $50 \mu \mathrm{l}$ of this protein solution were mixed with $200 \mu \mathrm{l}$ 1x Roti $^{\circledR}$-Nanoquant and incubated for $5 \mathrm{~min}$ at RT. Measurement of absorption was performed in the SynergyMx plate reader (BioTek, Friedrichshall, Germany) and calculated with the appropriate Gene5 software.

\subsubsection{Sodium Dodecyl Sulfate Polyacrylamide Gel Electrophoresis (SDS-PAGE)}

The NuPAGE ${ }^{\circledR}$ Pre-Cast Gel System (Life Technologies, Darmstadt, Germany) was used for the separation of proteins. It is a polyacrylamide gel system for high performance gel electrophoresis and is based on SDS-PAGE gel chemistry (Laemmli 1970). It consists of NuPAGE ${ }^{\circledR}$ Bis-Tris pre-cast gels and specifically optimized buffers which have an operating $\mathrm{pH}$ of 7.0. This neutral $\mathrm{pH}$ increases the stability in both proteins and gels, providing increased confidence in electrophoresis results.

The protein samples, usually $10-25 \mu$ g were mixed with 0.25 vol sample buffer (LDS Sample Buffer (4x), NuPAGE ${ }^{\circledR}$, Life Technologies, Darmstadt, Germany) and $10 \%$ DTT (reducing agent), denatured at $70^{\circ} \mathrm{C}$ for $10 \mathrm{~min}$, chilled on ice and centrifuged shortly before they were loaded onto the gradient gel (NuPAGE ${ }^{\circledR} 4-12 \%$ Bis-Tris Gel, Life Technologies, Darmstadt, Germany). Depending on the molecular weight of the proteins, gel electrophoresis was performed at $160 \mathrm{~V}$ for 1 to 2 hours using the $1 \mathrm{x}$ MES buffer (Life Technologies, Darmstadt, Germany). To determine the size of the proteins on a gel, 
$7 \mu \mathrm{l}$ of a pre-stained molecular weight standard (See Blue ${ }^{\circledR}$ Plus2, Life Technologies, Darmstadt, Germany) was loaded.

\subsubsection{Transfer of proteins from Polyacrylamide gels to PVDF membranes}

(Gershoni, Palade 1982); (Gershoni, Palade 1983)

After SDS-PAGE the proteins were transferred to a PVDF membrane using the semi-dry blotting technique. Therefore, a PVDF membrane (GE Healthcare, Freiburg, Germany) was cut to the size of the polyacrylamide gel and activated by rinsing in $100 \%$ Methanol for 5 - $10 \mathrm{sec}$. Subsequently, the membrane was equilibrated in transfer buffer IIa for approx. 10 min. Next, three sheets of Whatman GB003 filter paper (Schleicher \& Schull, Dassel, Germany) were, as well, cut to the size of the gel and soaked in transfer buffer IIa. Finally, three filter papers were placed on the anode $(+)$, followed by the PVDF membrane and the gel. The left three filter papers were applied to complete the sandwich model and the electro-blotter (Biometra, Göttingen, Germany) was closed, putting the cathode (-) on top. The transfer was carried out at $25 \mathrm{~V}$ and $220 \mathrm{~mA}$ for 30 min to 1.5 hours at RT, depending on the molecular weight of the proteins.

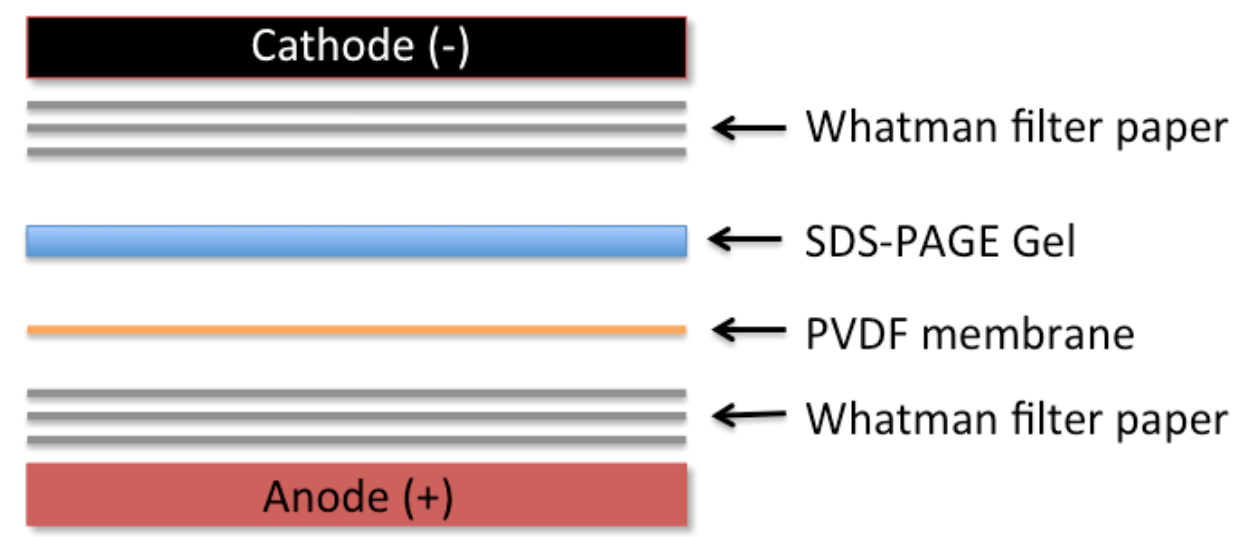

Fig. 2.1: Schematic overview of a setup of a semi-dry blot. Three sheets of Whatman filter paper are placed on the anode (-) followed by the PVDF membrane. The SDS-PAGE gel is placed on top of the membrane and covered with three sheets of Whatmann filter paper. The Cathode (-) is completing the sandwich model of the semi-dry blot. The applied voltage is forcing the proteins to migrate from the gel onto the PVDF-membrane. 


\subsubsection{Staining of polyacrylamide gels}

To check for proper protein transfer onto PVDF membranes, SDS-polyacrylamide gels were incubated for 1 to 4 hours in Coomassie Brilliant Blue staining solution at RT. Afterwards, SDS gels were de-stained in $\mathrm{H}_{2} \mathrm{O}$.

\subsubsection{Incubation of protein-bound membranes with antibodies}

To block unspecific binding sites, the membrane was incubated in blocking buffer (Western Blot) for 1 hour at RT. After extensive washing with washing buffer (Western Blot), the membrane was incubated with the primary antibody at a recommended antibody dilution in TBS-T at $4^{\circ} \mathrm{C}$ overnight. On the next day, the membrane was washed two times for $10 \mathrm{~min}$ with washing buffer (Western Blot) to wash off unbound antibodies and incubated with the appropriate secondary antibody in blocking solution (Western Blot) at RT for approx. 2 hours. After the incubation step, the membrane was washed three times for 15 min with washing buffer (Western Blot) and 5 min in TBS/T to fully remove any remaining milk. For the detection of the chemi-luminescent signals, the ECL Prime detection solution (GE Healthcare, Freiburg, Germany) was used. The membrane was placed on a plastic film, the detection solution was pipetted on the membrane and incubated for $5 \mathrm{~min}$, before the signals were captured using the Western Blot detection system (FlourChem ${ }^{\circledR}$ Q Alpha Innotech, Logan, USA).

For the detection of proteins using alkaline phosphatase (AP), the secondary antibodies were conjugated to alkaline phosphatase. After the membrane was incubated three times for 15 min with washing buffer (Western Blot) the membrane was washed with AP Buffer at RT for 5 min. Subsequently, the membrane was kept in the dark for the incubation in AP Staining solution to visualize protein bands directly on the PVDF membrane. The reaction was stopped by washing with washing buffer (Western Blot) and the membrane was dried. 
Tab. 2.1: Overview of the antibodies and the used dilutions in western blot analyses

\begin{tabular}{l|c|c}
\hline \multicolumn{1}{c|}{ Antibody } & $\begin{array}{c}\text { Dilution } \\
\text { Primary } \\
\text { antibody }\end{array}$ & $\begin{array}{c}\text { Dilution } \\
\text { Secondary } \\
\text { antibody }\end{array}$ \\
\hline Anti-phospho-Caldesmon (Ser-789) & $1: 2000$ & $1: 10000$ \\
\hline Anti- $\alpha$-Tubulin & $1: 10000$ & $1: 20000$ \\
\hline Anti-Caldesmon & $1: 1000$ & $1: 10000$ \\
\hline Anti-Integrin $\beta 1$ & $1: 1000$ & $1: 10000$ \\
\hline Anti-p44/42 MAPK (ERK1/2) & $1: 2000$ & $1: 10000$ \\
\hline Anti-phospho-p44/42 MAPK & $1: 2000$ & $1: 10000$ \\
(ERK1/2) (Thr202/Tyr204) & $1: 1000$ & $1: 10000$ \\
\hline Anti-Leupaxin (N-terminal) & &
\end{tabular}

\section{$2.16 \quad$ Co-Immunoprecipitation}

The Co-immunoprecipitation (Co-IP) is a method to study protein-protein interactions. A primary antibody directed against the protein of interest is immobilized to e.g. agarose or sepharose (beads) through protein $\mathrm{A} / \mathrm{G}$. This antibody binds specifically to the protein of interest in the protein lysate. In case there is an interaction with another protein, it will be co-precipitated during the purification process and can be detected in the western blot analyses. To analyze LPXN-CaD interaction, PC-3 cells were plated on T75 flasks and transfected with a pCMV-Myc-CaD or pCMV-Myc-LPXN plasmid, respectively. 48 hours after transfection the cells were lysed using the lysis buffer for protein (modified RIPA) and protein concentration was determined. For Co-IP $2 \mathrm{mg}$ of protein lysate were incubated with $10 \mu \mathrm{g}$ of the cmyc Tag antibody (Sigmal-Aldrich, Deisenhofen, Germany) at $4^{\circ} \mathrm{C}$ over night. On the next day, $20 \mu \mathrm{l}$ of resuspended protein A/G PLUS (Sanat Cruz Biotechnology, Heidelberg, Germany) was added and the mixture was incubated for 1 hour at RT to precipitate the cmyc fusion protein from the lysate. Centrifugation at $1.000 \mathrm{x}$ g pelleted the beads and the supernatant was removed. Next, the beads were washed three times in DPBS (PAN, AIdenbach, Germany) and boiled at $95^{\circ} \mathrm{C}$ for $10 \mathrm{~min}$, which reversed the binding of the antibody to the beads. The protein of 
interest containing supernatant was subjected to western blot analysis. In case of interaction, the interacting protein will be co-precipitated and can be detected in the subsequent western blot.

\subsection{Duolink ${ }^{\circledR}$ II Proximity Ligation Assay (PLA)}

The PLA is an immunocytochemical method that enables visualization of proteinprotein-interactions directly (in situ) in the fixed cell. Therefore, specific primary antibodies detect both proteins of interest. Importantly, both antibodies need to be raised in different species in order for the secondary antibody to bind to only one target. Next, specific secondary antibodies labeled with single stranded DNA detect these primary antibodies. The conjugated DNA sequences of the secondary antibodies are designated as plus (+) and minus (-). Two linker oligonucleotides that are complementary to the DNA sequences of the secondary antibodies help to form a circular structure. The length of the DNA fragments determines the proximity of the interacting proteins. Only when proteins are interacting $(<40 \mathrm{~nm})$ a hybridization of the DNA sequences is possible. The circular DNA structure is closed by a ligase-reaction. Now a DNA-polymerase can amplify the DNA-ring in rolling circle PCR. Finally, a fluorescently labeled probe detects the specific DNA sequence, which results in a fluorescent signal directly at the place of protein interaction, which can be detected by fluorescent microscopy. 


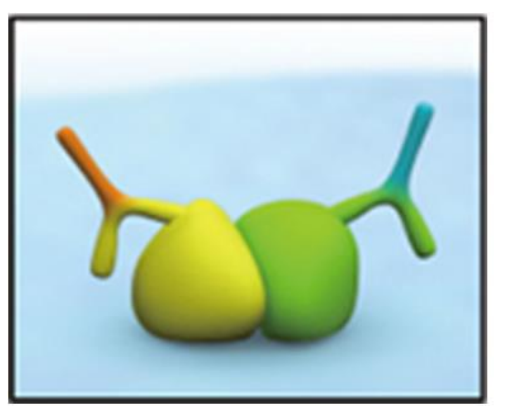

Primary antibodies from different species

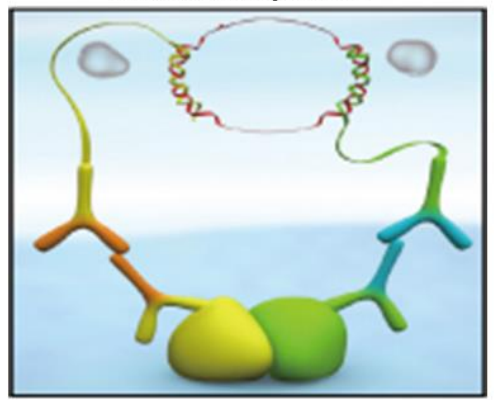

Ligation

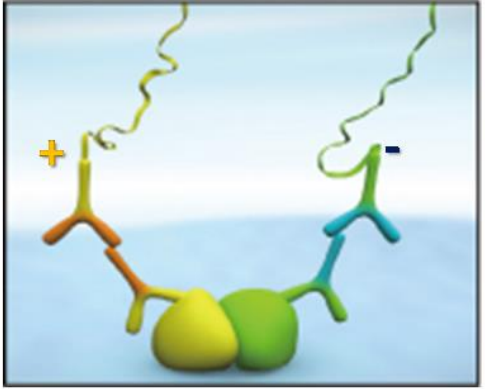

DNA-labeled secondary antibodies

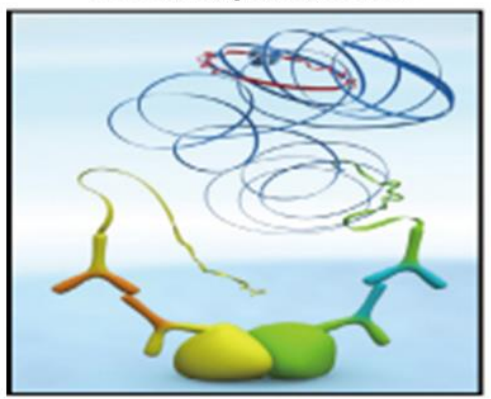

Rolling Circle PCR

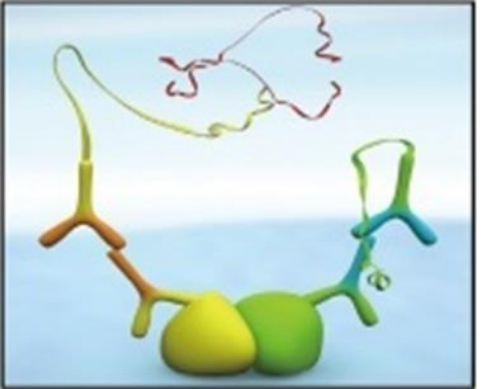

Linker Oligonucleotide

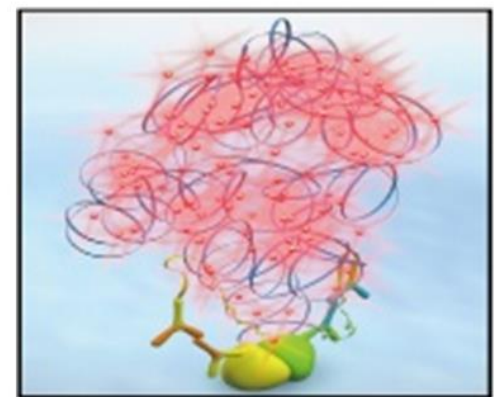

Fluorescent DNA probe

Fig. 2.2: Schematic overview of the Duolink ${ }^{\circledR}$ II Proximity Ligation Assay. 1) Proteins of interest are detected with primary antibodies raised in different species. 2) The primary antibodies are detected by specific, DNA-labeled secondary antibodies. 3) The addition of two linker oligonucleotides that are complementary to the DNA sequences of the secondary antibodies form a circular DNA structure. 4) The circular DNA structure is closed by a ligation-reaction. 5) A DNA-polymerase amplifies the DNA ring in a rolling circle PCR. 6) The amplified DNA fragments are detected by a fluorescently labeled DNA probe directly at the site of interaction. The fluorescent signal can be detected by fluorescent microscopy. Taken and modified from Duolink ${ }^{\circledR}$ II User Manual v. 21 2011, Olink Bioscience, Uppsala, Sweden.

\subsubsection{PLA on cells}

To simply test for interaction of two proteins, PLA was performed on PC-3 cells. Therefore, $4 \times 10^{4}$ cells were plated on a 4-well culture slide (Becton Dickinson $\mathrm{GmbH}$, Heidelberg, Germany). After 24 hours cells were washed gently with DPBS (PAN, Aidenbach, Germany) and cells were fixed using $3.7 \%$ formaldehyde in DPBS at RT for 20 min. Next the cells were washed with DPBS at RT for 10 min before the membrane was permeabilized with $0.1 \%$ Triton-X-100 solution for $10 \mathrm{~min}$. After this the cells were washed in DPBS at RT for 10 min. To block unspecific binding sites for antibodies, the cells were incubated in blocking buffer (PLA) for $30 \mathrm{~min}$. Subsequently, the primary antibodies were diluted in blocking buffer (PLA) to the corresponding concentration and 
incubated at $4^{\circ} \mathrm{C}$ over night. Primary antibodies used for PLA are listed in the following table.

Tab. 2.2: Overview of the antibodies and dilutions used for PLA

\begin{tabular}{l|c|l}
\hline \multicolumn{1}{c|}{ Antibody } & $\begin{array}{c}\text { Dilution } \\
\text { Primary } \\
\text { antibody }\end{array}$ & \multicolumn{1}{|c}{ Manufacturer } \\
\hline Anti-LPXN (283 G), mouse & $1: 150$ & ICOS Corp., Bothell, USA \\
\hline $\begin{array}{l}\text { Anti-phospho-Caldesmon Ser-789, } \\
\text { rabbit }\end{array}$ & $1: 150$ & $\begin{array}{l}\text { Santa Cruz Biotechnology, } \\
\text { Heidelberg, Germany }\end{array}$ \\
\hline $\begin{array}{l}\text { Anti-Caldesmon (8-L-Caldesmon), } \\
\text { mouse }\end{array}$ & $1: 150$ & $\begin{array}{l}\text { BD Transduction } \\
\text { Laboratories, Heidelberg, }\end{array}$ \\
\hline $\begin{array}{l}\text { Anti-p44/42 MAPK (ERK1/2), } \\
\text { rabbit }\end{array}$ & $1: 75$ & $\begin{array}{l}\text { Cell Signaling, Danvers, MA, } \\
\text { USA }\end{array}$ \\
\hline $\begin{array}{l}\text { Anti-phospho-p44/42 MAPK } \\
\text { (ERK1/2) (Thr202/Tyr204), rabbit }\end{array}$ & $1: 150$ & $\begin{array}{l}\text { Cell Signaling, Danvers, MA, } \\
\text { USA }\end{array}$ \\
\hline $\begin{array}{l}\text { Anti-Leupaxin (N-terminal), rabbit } \\
\text { (1:150 }\end{array}$ & $\begin{array}{l}\text { Sigma-Aldrich, } \\
\text { Deisenhofen, Germany }\end{array}$ \\
\hline
\end{tabular}

The continuous work was performed according to the manufacturer's instructions. To visualize the actin-cytoskeleton of the cells, $0.5 \mathrm{ng} / \mathrm{ml}$ FITC-conjugated Phalloidin was added to the solution of the secondary antibodies. Finally, the slide was dried at RT and kept in the dark until it was mounted in Vectashield/DAPI and sealed using nail polish. The slides were stored at $-20^{\circ} \mathrm{C}$. The pictures were captured using the confocal laserscanning microscope FluoView1000 (Olympus, Hamburg, Germany).

To analyze protein-protein interactions during migration, PLA was combined with a scratch assay. Therefore, PC-3 cells were plated to 4-well culture slides (Becton Dickinson GmbH, Heidelberg, Germany) and grown to confluence. When confluence was reached, a scratch was applied into the cell layer using a yellow pipet tip. After the scratch was applied, the cells were incubated for 18 hours until they were fixed in $3.7 \%$ formaldehyde. During that time cells were migrating into the scratch and relevant 
protein-protein interactions during cell migration can be analyzed. The subsequent steps followed the protocol of the manufacturer and did not differ from the described steps above.

\subsection{Electrophoretic mobility shift assay (EMSA)}

The EMSA is a method that is mainly used to identify DNA-binding proteins. The principle of EMSA uses the cage effect of the gel matrix, which slows down the migration speed of large complexes (e.g. DNA-bound proteins) through the gel, whereas small fragments (e.g. unbound oligonucleotides) are migrating faster. Usually, radioactively labeled DNA probes are used to visualize this gel shift, that's caused by the different size of DNA and DNA-protein complex.

\subsubsection{Casting of PAGE gels for EMSA}

For the EMSA experiment $4.8 \%$ PAGE gels were casted. Therefore, the following reaction was prepared for two gels:

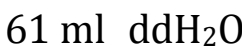

$9.0 \mathrm{ml}$ Rotiphorese ${ }^{\circledR}$ (40, Acrylamide/Bisacrylamide 29:1)

$3.6 \mathrm{ml} 5 \mathrm{xTBE}$

$1.5 \mathrm{ml} 10 \%$ APS

$75 \mu \mathrm{l}$ TEMED

After approx. 15 min the polymerization was completed. Next the gels were preequilibrated in 0.25x EMSA Running Buffer at $400 \mathrm{~V}$ for 2 hours.

\subsubsection{Radioactive labeling using 5'-fill-in reaction}

In the present study $\alpha$-33P dATP was used to label the double stranded oligonucleotides in a $5^{\prime}$-fill-in reaction.

$$
\begin{array}{ll}
1 \mu \mathrm{l} & \text { Oligonucleotide }(1 \mathrm{pmol} / \mu \mathrm{l}) \\
6 \mu \mathrm{l} & \alpha-33 \mathrm{PdATP}(10 \mathrm{mCu} / \mathrm{ml})
\end{array}
$$




\begin{tabular}{ll}
$5 \mu \mathrm{l}$ & $10 x$ Klenow Buffer \\
$1 \mu \mathrm{l}$ & Klenow-Polymerase \\
$\underline{37 \mu \mathrm{l}}$ & $\mathrm{dd}_{2} \underline{\mathrm{O}}$ \\
\hline
\end{tabular}

$50 \mu \mathrm{l}$

The reaction was incubated at RT for $25 \mathrm{~min}$. After incubation unbound $\alpha$-33PdATP nucleotides were removed from the reaction using the MicroSpin columns (GE Healthcare, Munich, Germany) according to the manufacturer's instructions.

\subsubsection{Gelshift reaction}

To analyze DNA-binding the following gel shift reaction was prepared in a master mix for all protein lysates:

$$
\begin{array}{ll}
4 \mu \mathrm{l} & \mathrm{ddH}_{2} \mathrm{O} \\
2.5 \mu \mathrm{l} & 5 \mathrm{x} \text { EMSA Shift Buffer } \\
0.5 \mu \mathrm{l} & \text { Poly(dIdC) }(2 \mathrm{mg} / \mathrm{ml}) \\
1.3 \mu \mathrm{l} & 100 \mathrm{mM} \text { DTT } \\
0.2 \mu \mathrm{l} & \text { radioactively labeled DNA probe }
\end{array}
$$

The individual gel shift reactions were prepared by adding $4.5 \mu \mathrm{l}$ of the protein lysate to $8.5 \mu \mathrm{l}$ of the Master mix. Depending on whether or not a supershift was induced $1-2 \mu \mathrm{l}$ of the corresponding antibody (STAT1-specific antibody C-24, Santa Cruz Biotechnology, Heidelberg, Germany) was added to the reaction. The mixture was incubated at RT for $20 \mathrm{~min}$ before it was loaded onto the pre-equilibrated gel and run at $400 \mathrm{~V}$. To estimate the migration speed of the complexes a bromphenol blue marker was loaded in parallel and the gel was stopped when it reached the end of the gel. Finally, the glass plates that held the gel were removed and the gel was placed on Whatman filter paper and vacuumdried. Binding activity was visualized with the phosphoimaging system Fujifilm FLA5100 (Fuji Life Science, Düsseldorf, Germany) using the computer programs Aida Image Analyzer v. 4.06 and TINA version 2.0 (Raytest, Straubhardt, Germany). 


\subsection{Cell biological methods}

\subsubsection{Cell culture of eukaryotic cells}

Adherent growing PC-3, DU145 and LNCaP cells were grown in commercially purchased RPMI 1640 medium (PAN-Systems GmbH, Nuremberg, Germany), which was supplemented with $1.2 \%$ Penicillin/Streptomycin (PAN-Systems GmbH, Nuremberg, Germany) and $10 \%$ fetal bovine serum (PAN-Systems GmbH, Nuremberg, Germany) in surface-treated cell culture flasks (Sarstedt, Nümbrecht, Germany). The cells were cultured at $37^{\circ} \mathrm{C}$ in a humidified incubator with $5 \% \mathrm{CO}_{2}$. Depending on the proliferation rate, cells were splitted one to two times per week. Therefore, cells were washed with DPBS (PAN-Systems GmbH, Nuremberg, Germany) and incubated at $37^{\circ} \mathrm{C}$ in a minimal amount of Trypsin/EDTA (PAN-Systems GmbH, Nuremberg, Germany) until the cells detached from the bottom of the culture flask. This process was controlled under an inverted microscope. After detachment, the Trypsin-reaction was stopped by the addition of growth medium and the cells were diluted in fresh culture medium by 1:5 to $1: 10$.

\subsubsection{Cryo-preservation and revitalization of eukaryotic cells}

Cells were grown to a confluence $>80 \%$, washed with DPBS (PAN) and trypsinized to detach cells from the bottom of the culture vessel. Cells were centrifuged at $500 \mathrm{x} g$ for 5 min to remove remaining trypsin and resuspended in an appropriate amount of culture medium containing $10 \%$ DMSO. The cells were then slowly cooled to $-80^{\circ} \mathrm{C}\left(1^{\circ} \mathrm{C} / \mathrm{min}\right)$ in a freezer using the Mr. Frosty (Thermo Scientific, Langenselbold, Germany). After seven days at $-80^{\circ} \mathrm{C}$ the cells were transferred to liquid nitrogen for long term storage. For revitalization, frozen cells were quickly thawed, spun down at $200 \mathrm{xg}$ for $3 \mathrm{~min}$ and the supernatant was aspirated. The cells were gently resuspended into pre-warmed culture medium, transferred into a culture flask and incubated overnight. On the next day, medium was changed in order to fully remove remaining amounts of DMSO. 


\subsubsection{Test for Mycoplasma contamination}

Mycoplasma contamination was routinely performed monthly using the MycoAlert ${ }^{\circledR}$ Mycoplasma Detection Kit (Lonza, Cologne, Germany) according to the manufacturer's instructions except that only half of the recommended amounts was used. This test is based on the measurement of the activity of mycoplasma-specific enzymes before and after application of specific substrates.

\subsubsection{Transfection of eukaryotic cells}

\subsubsection{Transfection of plasmids into eukaryotic cells}

Eukaryotic cells were transfected with plasmids in order to induce an overexpression of fusion proteins. The transfection reagent X-tremeGENE HP (Roche, Mannheim, Germany) is a unique non-liposomal reagent, free of animal-derived components and is able to form reagent-DNA complexes. Upon contact to the cell membrane the cell takes up the DNA. Only cells that were in a growth phase were used for transfection experiments. Cells were plated out on special 4-well slides and 6- or 96-well culture plates in RPMI 1640 medium, supplemented with $10 \%$ FBS, 24 hours before transfection. On the following day, when the cells reached a confluence of approx. 70 $90 \%$, DNA and transfection reagent were mixed according to the manufacturer's instructions, incubated for $30 \mathrm{~min}$ at RT and gently added directly into the culture medium. Afterwards, the cells were incubated for $24-72$ hours under normal culture conditions.

\subsubsection{Transfection of small interfering RNA (siRNA) into eukaryotic cells}

siRNAs are small RNA-oligonucleotides that specifically bind to a certain mRNA and mark them for degradation resulting in reduced gene expression (gene silencing).

For transfection of PCa cells with gene-specific siRNA-duplex-oligonucleotides the siRNA-transfection reagent OligoFectamine ${ }^{\mathrm{TM}}$ (Life Technologies, Darmstadt, Germany) was used according to the manufacturer's instructions with a final concentration of 
siRNA-duplexes of $80 \mathrm{mM}$. This reagent forms complexes of lipids and oligonucleotides that facilitate uptake of RNA molecules into mammalian cells. As a control PCa cells were transfected with siRNA-duplex-oligonucleotides against the luciferase gene of Photinus pyralis.

For the transfection PCa cells were plated either in T-25 or T-75 culture flasks and grown to $50 \%$ confluence. On the next day, the reaction was pipetted according to the manufacturer's instructions and incubated for $25 \mathrm{~min}$ at RT. During this time the cells were washed with DPBS (PAN, Aidenbach, Germany) and OptiMEM I (Life Technologies, Darmstadt, Germany) was added to each well before the transfection complexes were applied dropwise. Cells were then cultured in an incubator at $37^{\circ} \mathrm{C}$ and $5 \% \mathrm{CO}_{2}$ for 8 12 hours before the medium was replaced by normal growth medium. At different time points after transfection of the cells total protein or total RNA was isolated or cells were subjected to colony formation assays.

\subsection{Luciferase measurement}

For luciferase measurement the P.J.K. luciferase assay system (P.J.K., GmbH, Kleinblittersdorf, Germany) was used. To assess transfection efficiency cells were transfected with pRL-luc (Promega, Wisconsin, USA), which expresses the renilla reniformis luciferase. Before measurement the transfected cells were lysed using $40 \mu \mathrm{l}$ lysis-juice 2 (without detergents) per well. After 10 min incubation on ice $20 \mu \mathrm{l}$ of each well were transferred to two flat-bottomed lightproof (white) 96-well plates. Using the SynergyMx plate reader (BioTek, Friedrichshall, Germany) $25 \mu \mathrm{l}$ of Beetle Juice were pipetted to each well and luminosity was measured. For renilla luciferase $25 \mu$ of Renilla Juice were used.

\subsection{Analysis of reporter gene measurements}

The values obtained from the luciferase measurement were transferred to MS Excel (Microsoft) to calculate the transfection efficiency (firefly/renilla) and activation of the reporter gene. 


\subsection{Specificity protein-1 (Sp1) reporter assay}

To assess the activity of the Sp1 protein the Cignal ${ }^{\mathrm{TM}}$ Reporter Assay (Qiagen, Hilden, Germany) was used. For the reporter assay PC-3, DU145 and LNCaP cells were transfected with a combination of the reporter plasmid and the pEGFP-LPXN plasmid to assess the function of LPXN overexpression on SP1 activity. To analyze the effect of LPXN knockdown, a sequential transfection was performed consisting of a first transfection with siRNA against the LPXN gene and a second transfection with the reporter plasmid on the next day. All transfection mixtures contained a pCMV-Renilla luciferase plasmid to determine the transfection efficiency. Further steps were performed according to the manufacturer's instructions. Luciferase measurement was performed like described earlier.

\subsection{Functional analysis of eukaryotic cells}

\subsubsection{Colony formation assay}

In the present study colony formation assays were performed to determine the cell survival in response to irradiation. Depending on the irradiation dose siRNA-transfected or untransfected PC-3, DU145 and LNCaP cells were plated in normal growth medium on 6-well plates at the following densities 24 hours before they were exposed to different irradiation intensities:

$\begin{array}{ll}0 \text { Gy } & 200 \text { cells } \\ 2 \text { Gy } & 500 \text { cells } \\ 4 \text { Gy } & 750 \text { cells } \\ 8 \text { Gy } & 1000 \text { cells }\end{array}$

After colony formation after approx. 8 - 10 days the cells were fixed in ethanol for 20 min and stained using a hematoxylin/eosin staining solution. The colonies were counted on a light table. 


\section{$2.24 \quad$ In vivo-studies}

\subsubsection{Generation of a conditional LPXN knockout mouse model}

Mammalian embryos possess the ability to incorporate cells of foreign origin and produce chimeras. This ability has been used in a variety of purposes including the generation of knockout mouse models. For the generation of a conditional LPXN knockout mouse model, mutant ES cells were purchased from the European Conditional Mouse Mutagenesis program (EuCOMM). These ES cells were carrying a targeted trap construct in the LPXN gene, leaving the opportunity for the generation of knockout, conditional knockout and reporter mouse lines.

\subsubsection{FLP recombination of LPXN knockout ES cells}

In order to achieve the conditional allele, the reporter/resistance cassette has to be removed by FLP recombination. This work was done in close cooperation with Prof. Dr. rer. nat. Ibrahim Adham (Department of Human Genetics, Göttingen, Germany). In brief, the mouse ES cells were cultured on a feeder layer in normal ES cell medium and transfected with the pCAGGS-FLPe plasmid to express the FLP-recombinase. Recombination was checked on isolated DNA. In case of positive recombination ES cells were used for blastocyst injection.

\subsubsection{Blastocyst injection}

The injection of the LPXN knockout ES cells was performed in cooperation with Dr. Ursula Fünfschilling (Max Planck Institute of Experimental Medicine, Göttingen, Germany). For the blastocyst injection wild type C57BL/6 females were sacrificed and injected blastocysts were transplanted into C57BL/6 foster mothers. 


\section{$3 \quad$ Results}

\subsection{The interaction of LPXN with caldesmon and it's relevance for PCa cell migration}

In previous studies of our research group the actin-binding protein caldesmon (CaD) was identified as an interaction partner of LPXN (von Hardenberg, 2010). In addition, we could show that downregulation of LPXN decreased the phosphorylation of $\mathrm{CaD}$ at S534 corresponding to the low molecular mass isoform of CaD (l-CaD).

In the present study we were interested in the confirmation of the interaction of LPXN and CaD. Furthermore, the relevance of this interaction regarding the de-/stabilization of the actin-cytoskeleton was investigated. In addition, we analyzed how the changes in CaD phosphorylation status are implicated in this process.

\subsection{Confirmation of LPXN-CaD interaction}

\subsubsection{Co-immunoprecipitation of LPXN and l-CaD}

In the aforementioned studies the interaction of LPXN and CaD was detected by a GSTpulldown experiment. To confirm this interaction in cells a co-immunoprecipitation was conducted on whole protein lysate of PC-3 cells, isolated 48 hours after transfection with pCMV-myc-CaD or pCMV-myc-LPXN, respectively. Precipitation with a CaD-specific antibody followed by immunoblotting using a LPXN-specific antibody showed a specific band at $45 \mathrm{kDa}$, which was not detectable in the pCMV-myc transfected control sample, meaning that LPXN was successfully precipitated from the lysate (Fig. 3.1). In addition, reciprocal co-immunoprecipitation with a LPXN-specific antibody and immunoblotting using a CaD-specific antibody (77kDa) verified the interaction of LPXN and CaD (Fig. 3.1). 


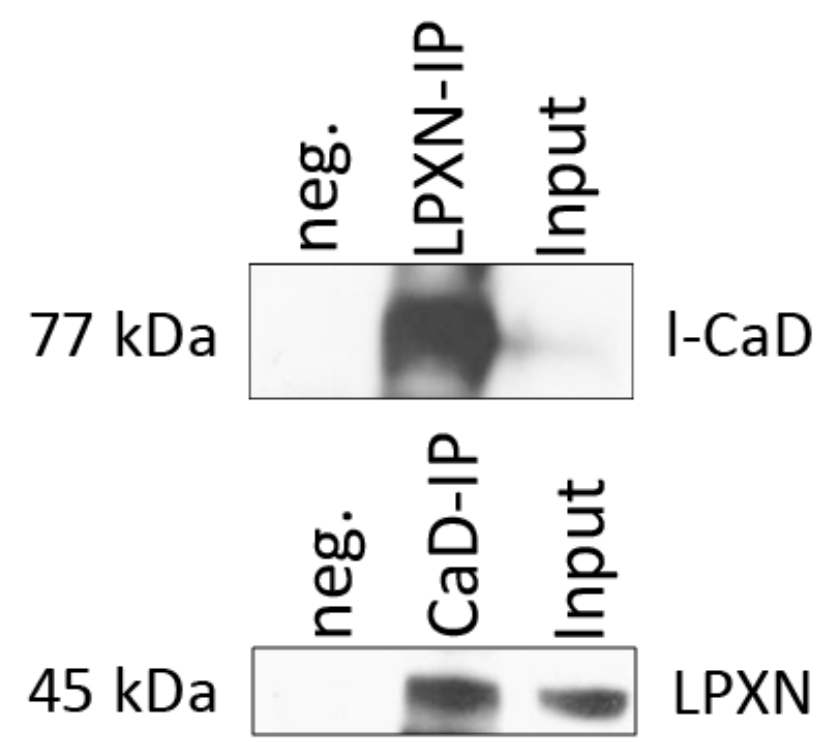

Fig. 3.1: The actin-binding protein CaD is interacting with LPXN. The interaction was visualized by immunoprecipitation of $\mathrm{CaD}$ from $\mathrm{PC}-3$ whole protein lysate with a CaD-specific antibody and immunoblotting using a LPXN-specific antibody (upper panel). Reciprocal co-immunoprecipitation with a LPXN-specific antibody and immunodetection using a CaD-specific antibody confirmed the interaction (bottom panel). Antibodies detected specific bands for the low molecular mass isoform of CaD (l-CaD) at $77 \mathrm{kDa}$ and for LPXN at $45 \mathrm{kDa}$ in the samples as well as in the input controls. As negative control lysate from pCMV-myc transfected cells was used (modified from Dierks et al. 2015).

\subsubsection{In situ Proximity ligation assay (PLA) of LPXN and CaD}

In order to verify LPXN and CaD interaction an in situ proximity ligation assay (PLA) using rabbit anti-LPXN and mouse anti-purified-l-CaD antibodies was performed. The in situ PLA could confirm the interaction of LPXN and CaD in both PC-3 and DU145 PCa cells. Interactions are indicated as red dots, DNA was stained using 4',6-diamidin-2phenylindol (DAPI) and F-actin was visualized by fluorescein isothiocyanate (FITC)conjugated phalloidin (Fig. 3.2). PC-3 and DU145 cells were analyzed by confocal laser scanning microscopy. Interactions showed a very evenly distributed pattern in nonstimulated and non-migrating cells. 

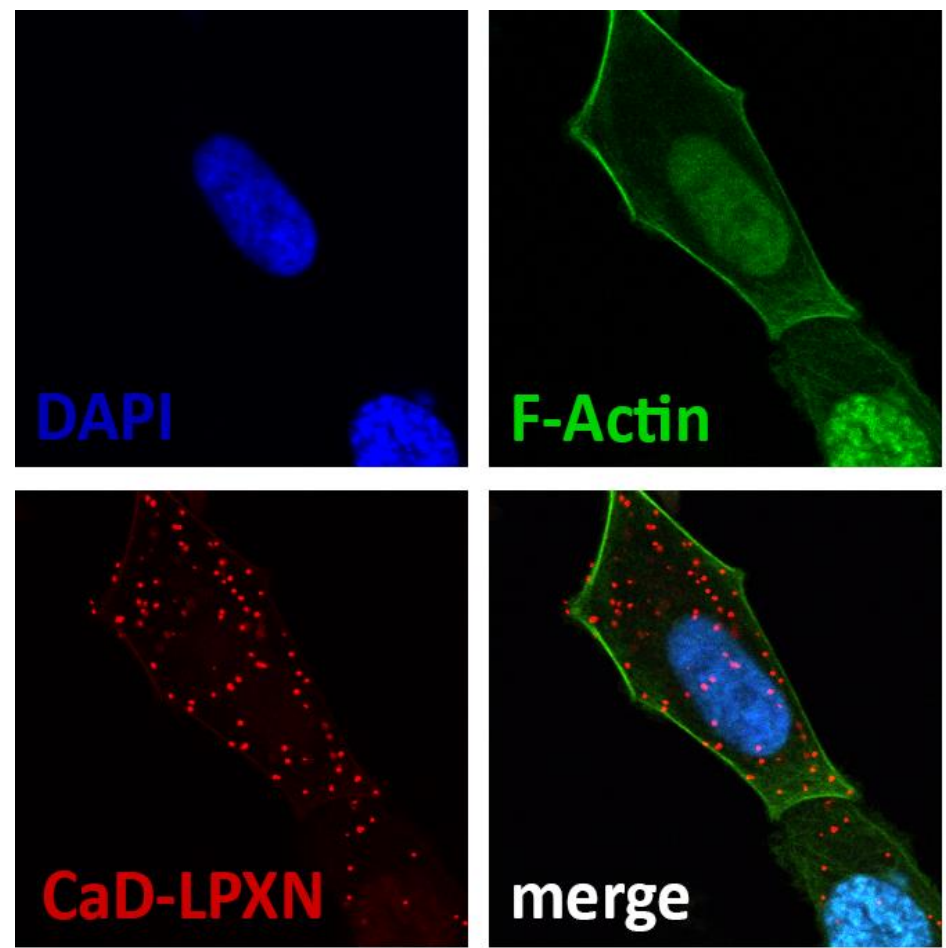

Fig. 3.2: In situ Proximity ligation assay (PLA) of PC-3 cells showing LPXN-CaD interaction. To visualize LPXN-CaD interaction, PC-3 cells were plated on glass culture slides, fixed and subjected to in situ PLA using polyclonal rabbit anti-LPXN and monoclonal mouse anti-purified l-CaD antibodies. LPXN-CaD interactions are visualized as red dots, confirming an interaction of CaD and LPXN. FITC-conjugated phalloidin stained F-actin (green), whereas DNA was stained with DAPI (blue). Cells were analyzed by confocal laser scanning microscopy using the Olympus FluoView1000 confocal scanning microscope at a 600-fold magnification. For better visualization, the picture was zoomed in.

\subsection{Increased interaction of LPXN and CaD during PCa cell migration}

To further analyze LPXN-CaD interaction in situ, PC-3 and DU145 cells were plated on 4well glass culture slides and grown to confluence. For the next steps we combined a scratch assay with the PLA technique. Therefore, the confluent cell layer was scratched using a yellow pipet tip. After 18 hours of migration into the scratch cells were fixed in $3.7 \%$ formaldehyde followed by in situ PLA using rabbit anti-leupaxin and mouse antipurified l-CaD antibodies. F-actin was stained using FITC-conjugated phalloidin and cells were mounted in DAPI-containing medium to visualize nuclei. Confocal laser scanning microscopy revealed that by combination of these two methods there were increased 
interactions of both proteins during cell migration of PCa cells as compared to cells that resided in the confluent cell layer and did not migrate (Fig. 3.3).

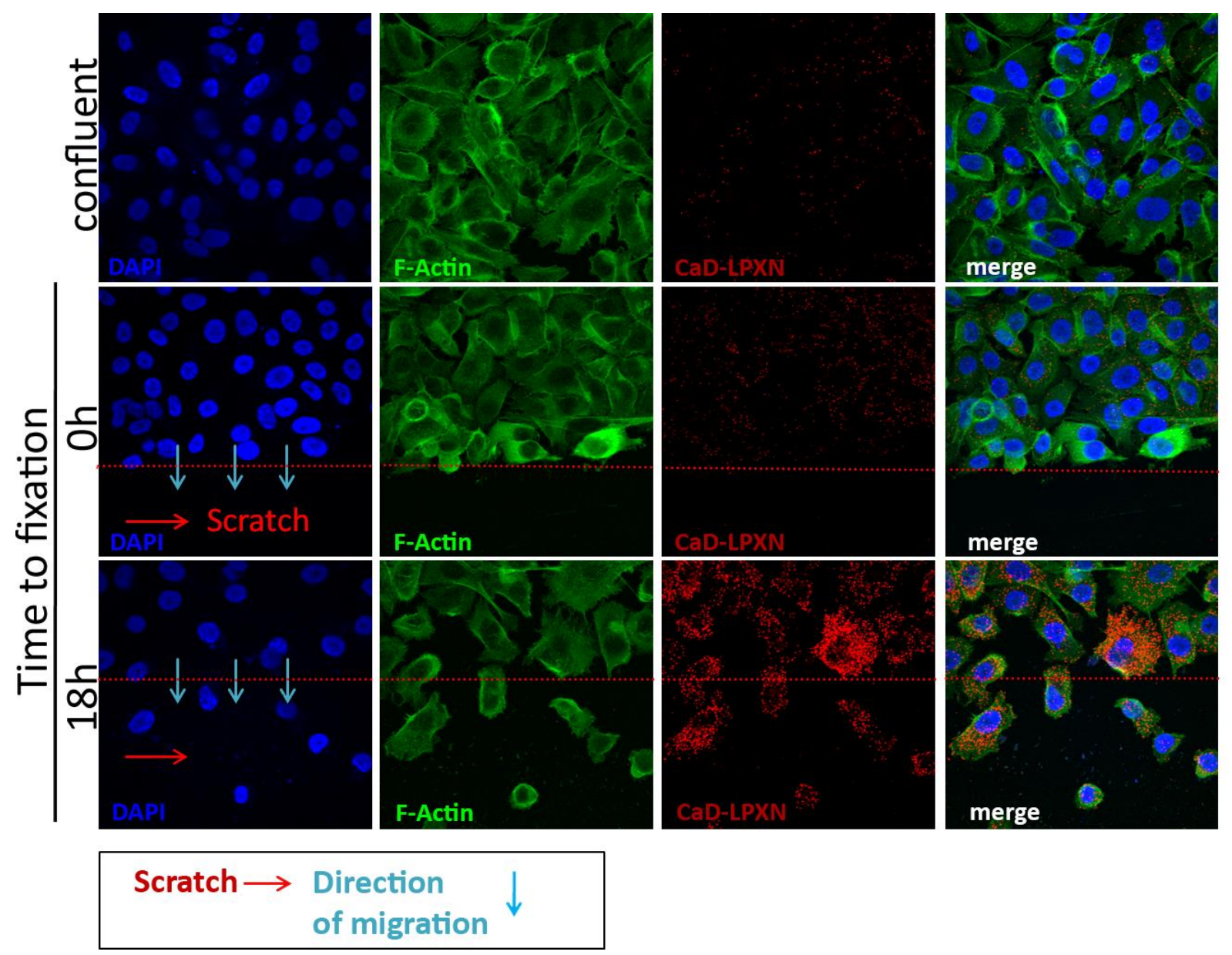

Fig. 3.3: Increased interaction of LPXN and CaD during migration. To investigate LPXN and CaD interaction during migration, a combination of scratch assay and in situ PLA was performed. To this end, PC-3 cells were plated on glass culture slides, grown to confluence and a scratch was applied into the cell layer. Cells were fixed after 18 hours and in situ PLA was performed using specific mouse anti-LPXN and rabbit anti-CaD antibodies. Confluent cells did not show high numbers of interaction, whereas cells that migrated into the scratch showed increased interactions. Blue arrows indicate the direction of migration, the scratch is depicted by the red arrow. Nuclei and F-actin were stained using DAPI and FITC-conjugated phalloidin, respectively. Images were captured at a 600 -fold magnification using the Olympus FluoView1000 confocal scanning microscope (modified from Dierks et al. 2015). 


\subsection{Increased interaction of LPXN and phosphorylated CaD during PCa cell migration}

After downregulation of LPXN expression we could show diminish phosphorylation of CaD pointing towards a regulation of CaD phosphorylation by LPXN (von Hardenberg, 2010). To this end, we performed in situ PLA using mouse anti-LPXN and a phosphorylation site-specific rabbit anti-phospho-CaD S789 antibodies to see if phosphorylated $\mathrm{CaD}$ shows a different interaction pattern with LPXN than unphosphorylated CaD. For this purpose, PC-3 and DU145 cells were plated on 4-well glass culture slides and the experiment was performed as described in Materials and Methods 2.17. S789-phosphorylated CaD did not display different interaction patterns with LPXN during migration of PCa cells compared to unphosphorylated CaD (Fig. 3.4). Likewise, migrating cells had increased interactions, whereas confluent and nonmigrating cells only showed weak interactions (indicated by red dots).

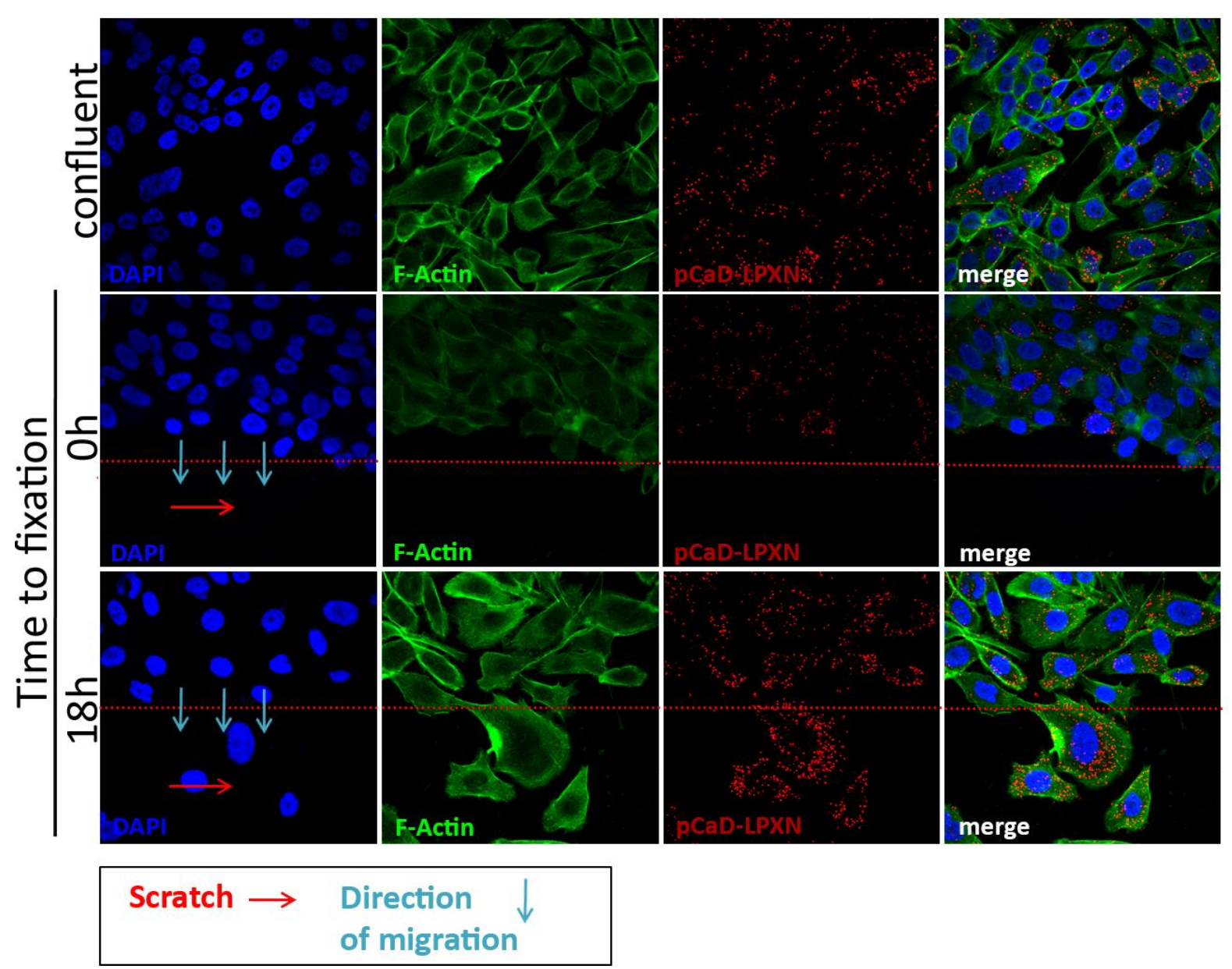


Fig. 3.4: Increased interaction of LPXN and pCaD during migration. Investigation of LPXN and pCaD interaction during migration was carried out by a combination of scratch assay and in situ PLA. Therefore, PC-3 cells were plated on glass culture slides, grown to confluence and a scratch was applied into the cell layer. Cells were fixed after 18 hours and in situ PLA was performed using specific mouse anti-LPXN and rabbit anti-phospho-CaD S534 antibodies. Confluent cells showed only weak interactions, whereas cells that migrated into the scratch showed increased interactions. Blue arrows indicate direction of migration, the scratch is depicted by the red arrow. Nuclei and F-actin were stained using DAPI and FITC-conjugated phalloidin, respectively. Images were captured at a 600-fold magnification using the Olympus FluoView1000 confocal scanning microscope (modified from Dierks et al. 2015).

\subsection{Localization of pCaD-LPXN interaction during migration of PCa cells}

In order to analyze the subcellular localization of the CaD/pCaD-LPXN interactions during PCa cell migration, PC-3 and DU145 cells were plated on glass culture slides and a scratch was applied into the confluent cell layer. After 18 hours cells were fixed and the in situ PLA was performed. Migrating cells clearly showed interactions of LPXN and $\mathrm{pCaD}$ in areas of dynamic actin structures, whereas they were nearly absent from the leading edge of the migrating cell, where the actin-cytoskeleton needs to be stabilized (Fig. 3.5). 

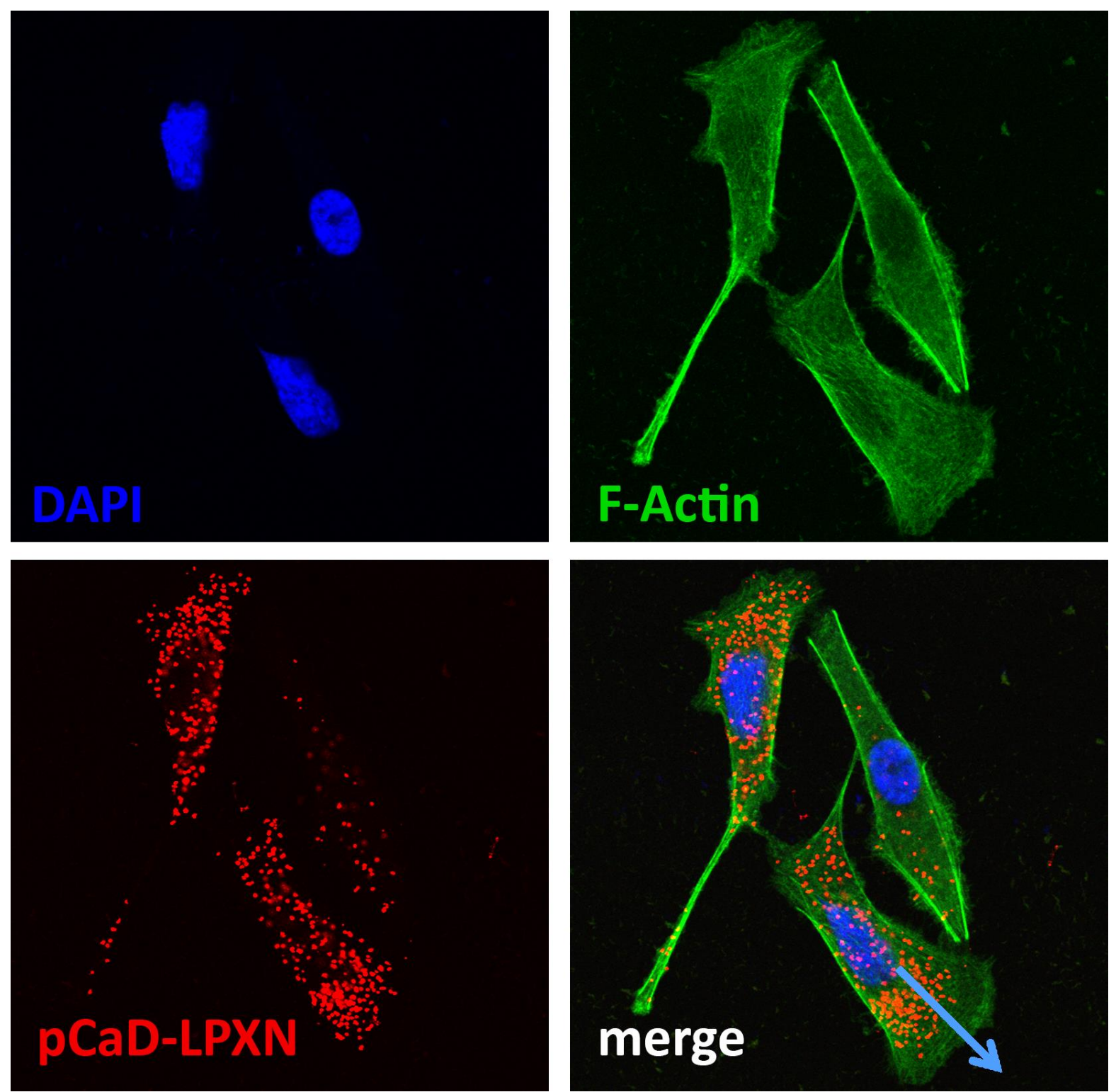

Fig. 3.5: In situ PLA visualized the localization of pCaD-LPXN interactions in migrating PC-3 cells. pCaD interaction with LPXN on celllular level was analyzed by in situ PLA on migrating PC-3 cells. Cells were grown on glass culture slides to about $50 \%$ confluence. On the next day the cells were fixed and in situ PLA was performed using specific mouse anti-LPXN and rabbit anti-phospho-CaD S534 antibodies.

As indicated by red dots, interactions of $\mathrm{pCaD}$ and LPXN during migration are located to regions of dynamic actin structures. At the leading edge of the cells as well as at stabilized actin filaments there were no interactions detectable (red dots). Nuclei were visualized by DAPI staining. F-actin was stained using FITC-conjugated phalloidin. The arrow indicates direction of migration. Images were captured using the Olympus FluoView1000 confocal scanning microscope at a 600-fold magnification and zoomed to visualize migrating cells (modified from Dierks et al. 2015). 


\subsection{LPXN interacts with the extracellular signal-regulated kinase (ERK)}

F-actin-binding of l-CaD in non-muscle cells is mainly regulated in a phosphorylationdependent manner. Since LPXN does not carry any kinase activity, phosphorylation of $\mathrm{CaD}$ has to be carried out by a kinase that is recruited to phosphorylate CaD during cell migration.

From the literature there are several kinases known to phosphorylate CaD specifically on the phosphorylation site S789 of h-CaD / S534 of l-CaD responsible for actin affinity. Among these kinases are the p38 mitogen-activated protein kinase (MAPK) and the extracellular signal-regulated kinase (ERK) (Jeon 2009). From previous experiments including the antibody array KAM 850 (Kinexus, Canada) we know that TGF-beta activated kinase 1 (TAK1) is downregulated after LPXN knockdown (von Hardenberg, 2010). TAK1 was shown to activate JNK and p38 MAPK through phosphorylation. However, only JNK and ERK showed reduced phosphorylation after LPXN knockdown, and ERK was already described to be associated with migration-dependent phosphorylation at the actin binding sites of CaD (D'Angelo et al. 1999, Roman et al. 2014). In addition, previous experiments in our research group found that LPXN knockdown resulted in reduced phosphorylation of ERK (Kaulfuß, 2006). Therefore, we hypothesize that ERK is the kinase responsible for LPXN-mediated phosphorylation of $\mathrm{CaD}$ during cell migration. To test our hypothesis we investigated a putative interaction of ERK and LPXN in both PC-3 and DU145 cells using in situ PLA. Therefore, PC-3 and DU145 cells were plated on 4-well glass culture slides and fixed in $3.7 \%$ formaldehyde after 24 hours of culture in normal culture medium. Figure 3.6 clearly shows the interactions of LPXN and ERK, as indicated by the red dots. 

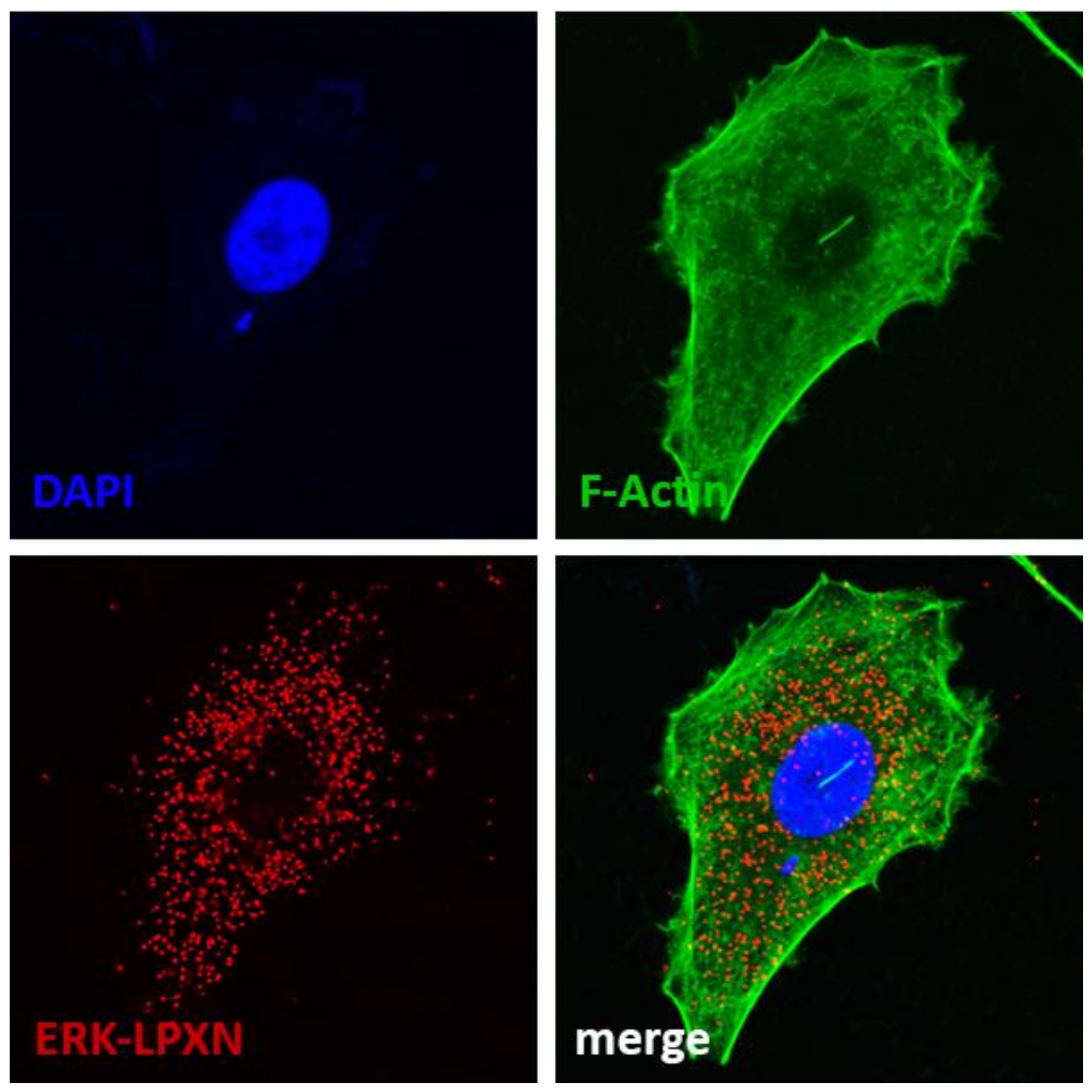

Fig. 3.6: In situ PLA of PC-3 cells showing interaction of ERK and LPXN. To test whether ERK is the kinase that is mediating the phosphorylation of $\mathrm{CaD}$ an in situ PLA was performed using polyclonal rabbit anti-ERK1/2 and mouse anti-LPXN antibodies. PC-3 and DU145 cells were plated on glass culture slides, fixed in $3.7 \%$ formaldehyde and in situ PLA was performed. Red dots clearly indicate interaction of ERK and LPXN. F-actin was stained using FITC-conjugated phalloidin (green). DAPI visualized the nucleus (blue). Images were captured at a 600-fold magnification using the Olympus FluoView1000 confocal scanning microscope and zoomed in for better visualization.

\subsection{Basal expression of ERK in PCa cell lines PC-3, DU145 and LNCaP}

The deregulation of the MAP kinase pathway is a common feature in a wide variety of cancers. As ERK is a major component of this pathway and seems to be involved in the LPXN-mediated phosphorylation of CaD, the expression and activation status of ERK was analyzed in PCa cell lines. Therefore, whole protein lysates of PC-3, DU145 and 
LNCaP cells were immunoblotted using specific anti-ERK and anti-pERK antibodies. Among all cell lines, there was a quite equal expression of ERK, whereas only DU145 showed activated levels of ERK (pERK) (Fig. 3.7).

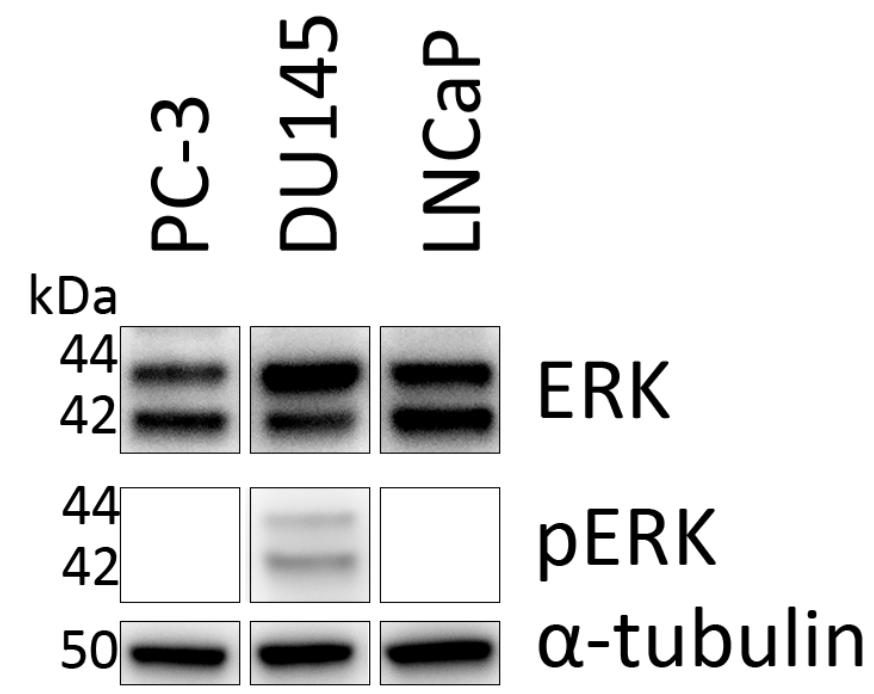

Fig. 3.7: Basal ERK expression and activation status in PCa cells. Immunoblotting of whole protein lysate of PC-3, DU145 and LNCaP cells using specific-ERK and pERK antibodies revealed equal levels of ERK in all cell lines. Among the PCa cell lines tested only DU145 cells showed activated ERK (pERK). Immunostaining of $\alpha$-tubulin ensured equal protein loading.

\subsection{Increased interaction of LPXN and ERK during PCa cell migration}

Having shown a clear interaction of LPXN and phospho-CaD and of LPXN and ERK during migration of PC-3 cells, we analyzed whether LPXN-ERK interaction is also induced by and relevant for migration. Therefore, cells were plated on 4-well glass culture slides and scratches were applied into a confluent PC-3 cell layer. After 18 hours, cells were fixed and in situ PLA was carried out using specific mouse anti-LPXN and rabbit anti-ERK antibodies. Similar to the pattern of $\mathrm{LPXN}-\mathrm{CaD} / \mathrm{pCaD}$ interactions an increased interaction of LPXN and ERK in migrating cells compared to confluent or nonmigrating cells (Fig. 3.8) was observed. Interestingly, we also found the same interaction pattern for LPXN and activated ERK (pERK) (Fig. 3.9). 


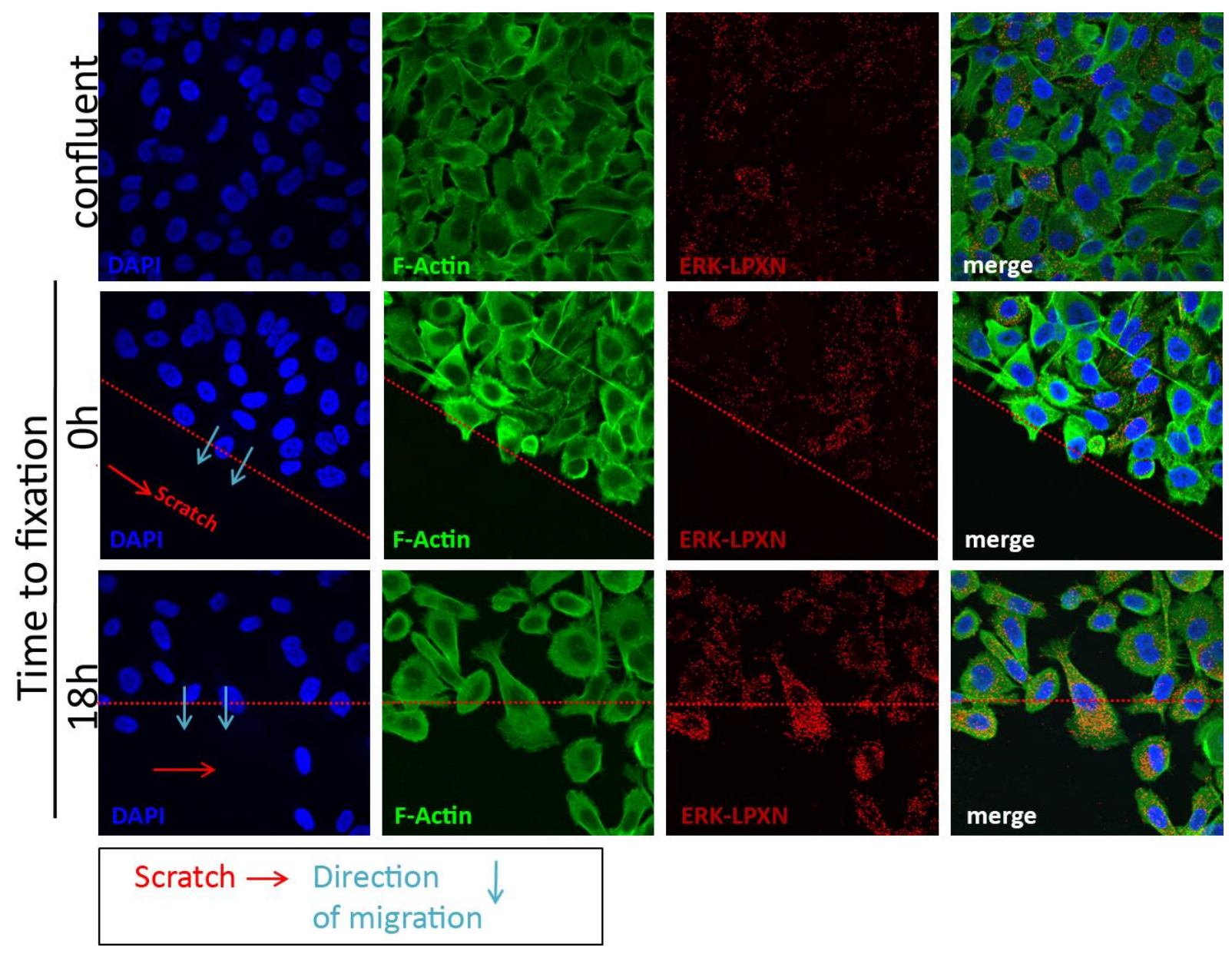

Fig. 3.8: In situ PLA of migrating PC-3 cells showing ERK-LPXN interactions during PCa cell migration. To analyze ERK-LPXN interaction during migration of PC-3 cells, a combination of a scratch assay and in situ PLA was performed. Therefore, PC-3 cells were plated on glass culture slides, grown to confluence and a scratch was applied into the cell layer. After 18 hours, cells were fixed and an in situ PLA was performed using specific mouse anti-LPXN and polyclonal rabbit anti-ERK1/2 antibodies. Migrating cells showed increased interactions compared to non-migrating and confluent cells indicated by red dots. Nuclei (blue) and F-actin (green) were visualized using DAPI and FITC-conjugated phalloidin, respectively. Images were captured at a 600-fold magnification using the Olympus FluoView1000 confocal scanning microscope (modified from Dierks et al. 2015). 


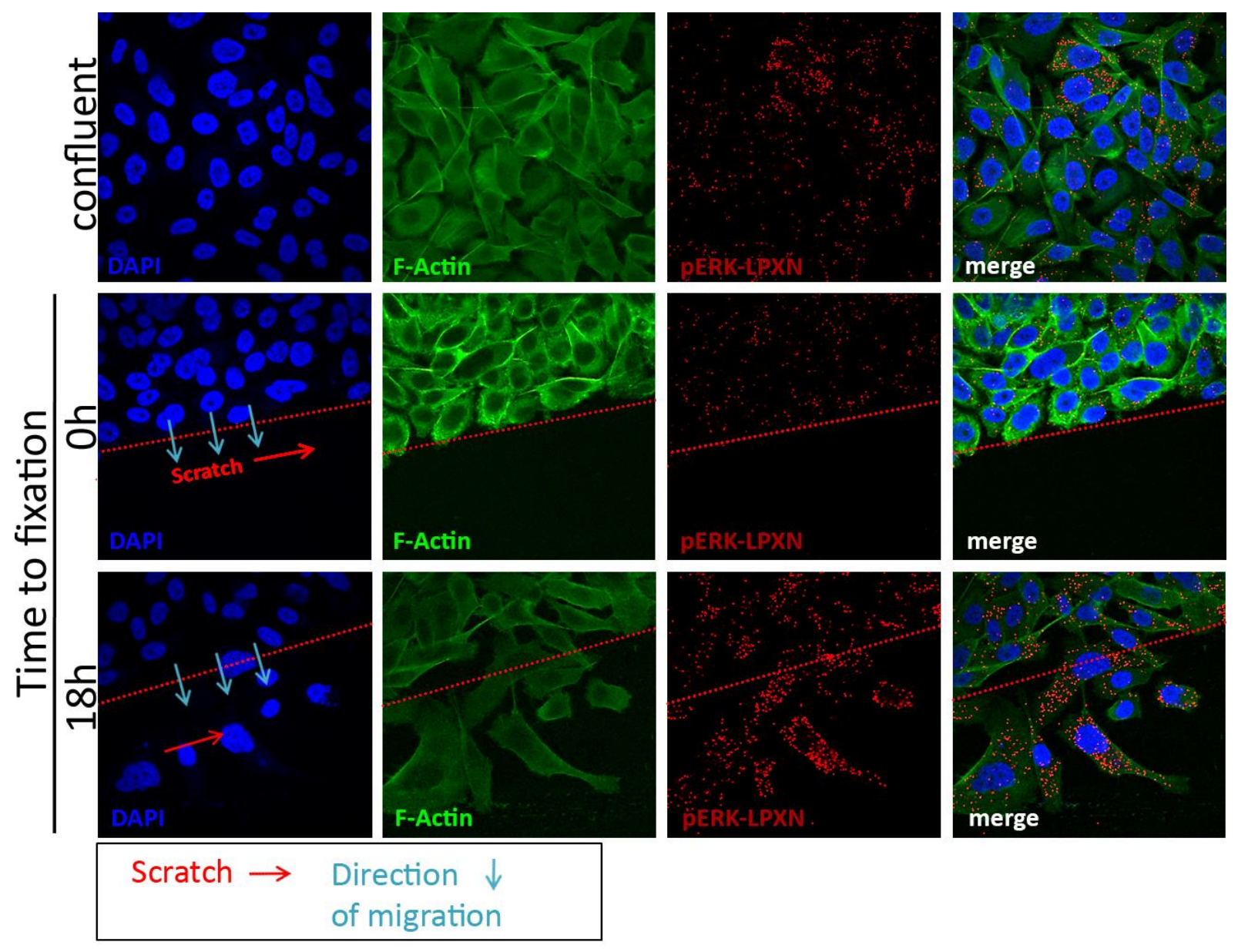

Fig. 3.9: In situ PLA of migrating PC-3 cells showing increased interaction of LPXN and pERK. LPXN and pERK interaction was analyzed by a combination of a scratch assay and in situ PLA. PC-3 cells were plated on glass culture slides and grown to confluence before a scratch was applied into the cell layer. After 18 hours, cells were fixed and an in situ PLA was performed using specific anti-LPXN and antipERK1/2 antibodies. Migrating cells showed increased interactions compared to non-migrating and confluent cells indicated by red dots. Nuclei (blue) and F-actin (green) were visualized using DAPI and FITC-conjugated phalloidin, respectively. Images were captured at a 600 -fold magnification using the Olympus FluoView1000 confocal scanning microscope (modified from Dierks et al. 2015).

\subsection{LPXN mediates phosphorylation status of CaD through interaction with ERK}

To further investigate if ERK is the kinase that is recruited to phosphorylate CaD during migration, PC-3 cells were treated with a combination of the MEK1 (mitogen-activated protein kinase kinase 1)-specific inhibitor PD98059, which blocks activation of ERK by its upstream kinase MEK1 and the epidermal growth factor (EGF) to induce ERK activation. In the subsequent western blot analysis of the protein lysates using a phosphorylation site-specific anti-phospho-CaDS789 antibody reduced levels of pCaD in 
PD98059 treated cells were observed, which could not be prevented or attenuated by EGF stimulation (Fig. 3.10). Notably, reduction of CaD phosphorylation could not be further enhanced by a downregulation of LPXN expression. Levels of unphosphorylated CaD remained equal. Reduced pERK levels in PD98059 treated cells indicated the functionality of the inhibitor. It is worth to note that ERK activation was reduced after LPXN knockdown, which underlines the hypothesis that ERK is mediating the phosphorylation of CaD in a LPXN-dependent manner.

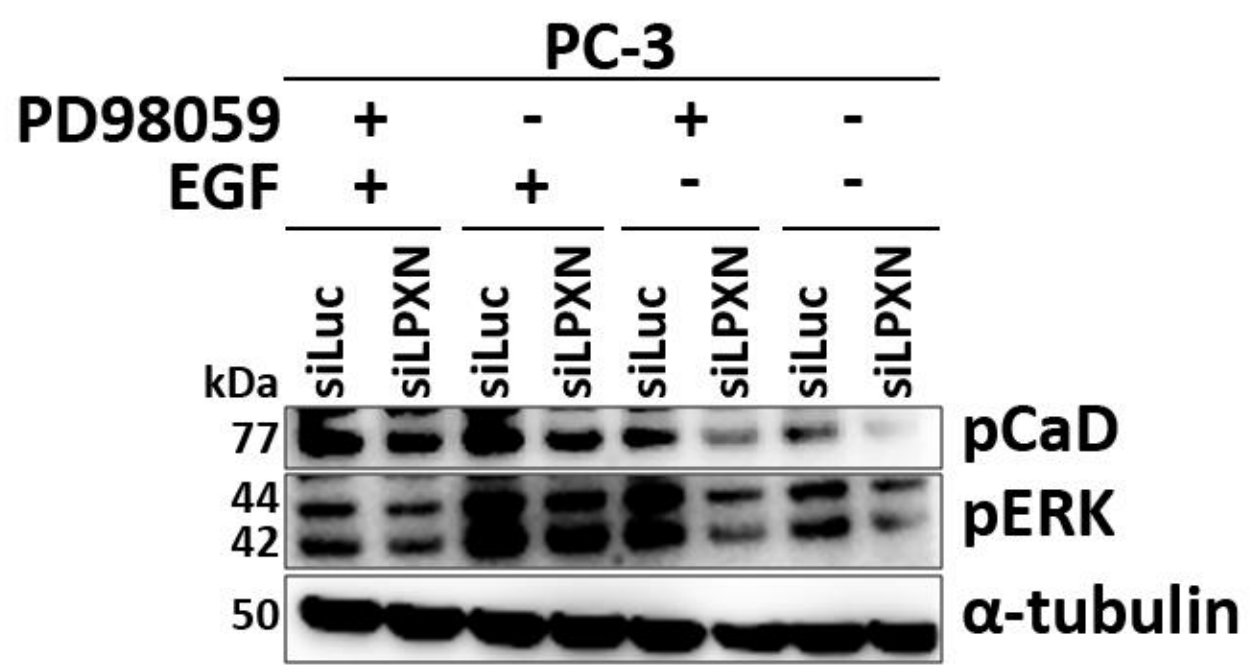

Fig. 3.10: Phosphorylation status of CaD in PC-3 cells after LPXN knockdown and MEK-1 inhibition. PC-3 cells plated on 6-well paltes and transfected with siRNA against the LPXN (siLPXN) or luciferase (siLuc) gene as a control. On the next day the cells were treated with the MEK1-specific inhibitor PD98059 for 1 hour, or EGF or a combination of both. Protein lysates were isolated 20 min after EGF stimulation and subjected to western blot analyses using specific antibodies for phospho-CaD S789, phospho-ERK1/2 and $\alpha$-tubulin. Knockdown of LPXN reduced phosphorylation levels of CaD and notably of ERK. Prevention of CaD-phosphorylation by inhibition of MEK-1 using the PD98059 inhibitor also showed reduced phosphorylation of $\mathrm{CaD}$, but had no additional effect to LPXN knockdown (modified from Dierks et al. 2015).

\subsection{The influence of LPXN on PCa cell adhesion}

In previous studies from our research group LPXN was shown to exert dramatic effects on the adhesion of PCa cells (von Hardenberg, 2010). Integrins are major components of the adhesion process. Therefore, the expression levels of several integrins were investigated. Out of this analyses the deregulation of several integrins was observed, 
especially the expression of the integrin $\beta 1$ gene (ITGB1), a major player of cell adhesion was correlating with LPXN expression (Hitzing, 2010).

\subsubsection{Expression of ITGB1 in the established PCa cell lines PC-3, DU145 and LNCaP}

The expression of ITGB1 was analyzed in the established PCa cell lines PC-3 DU145 and LNCaP using real time RT-PCR as well as western blot analysis. These analyses revealed that on the RNA as well as on the protein level the androgen-independent and highly invasive cell lines PC-3 and DU145 showed the highest expression of ITGB1. Whereas, androgen-dependent and non-invasive LNCaP cells showed rather weak expression levels compared to PC-3 and DU145 cells (Fig. 3.11).

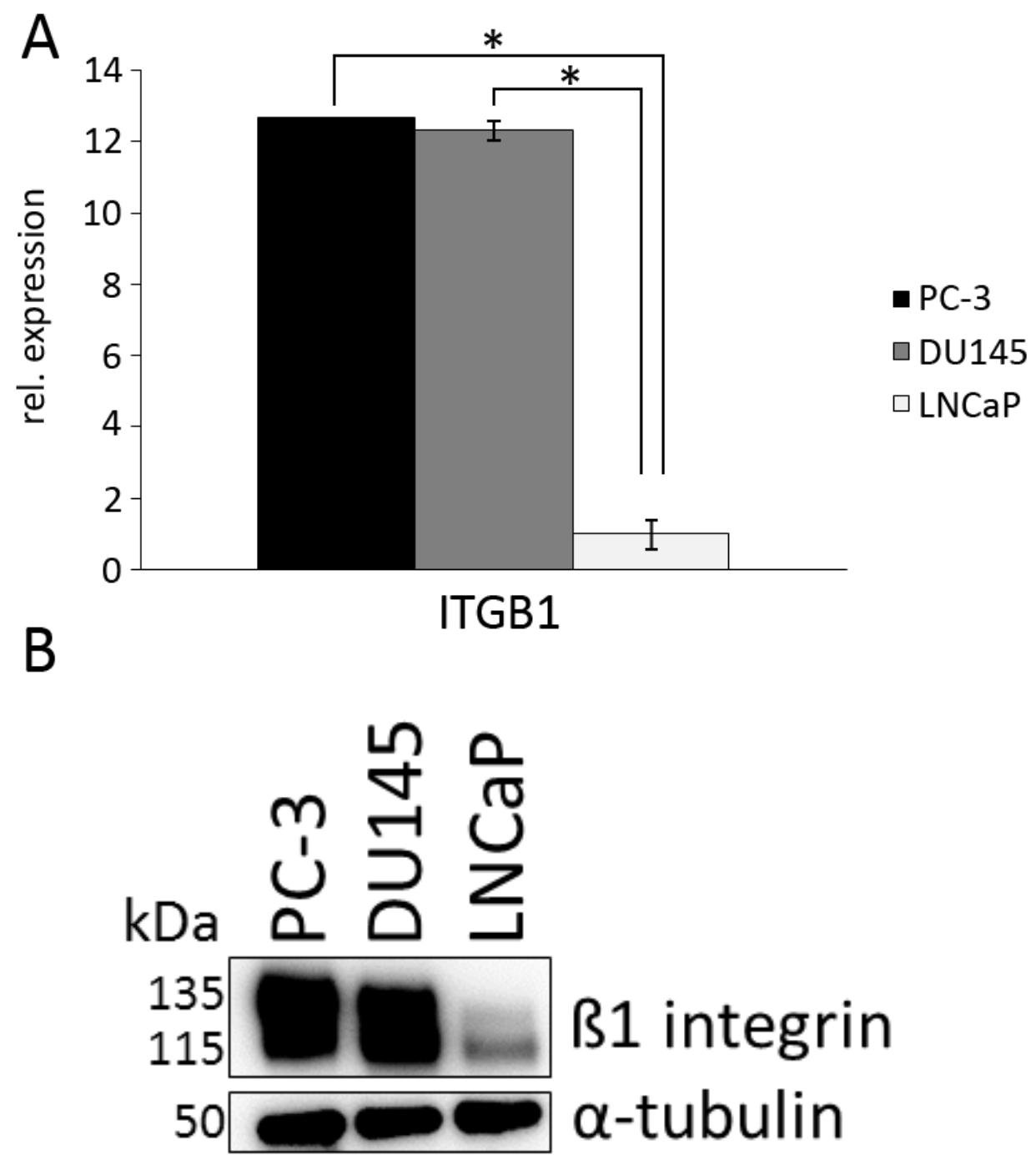


Fig. 3.11: Basal $\beta 1$ integrin expression in the PCa cell lines PC-3, DU145 and LNCaP. (A) PC-3, DU145 and LNCaP cells were plated on 6-well plates and total RNA was isolated, reverse transcribed and subjected to qRT-PCR. PC-3 and DU145 showed an approx. 12-fold increase of $\beta 1$ integrin expression compared to LNCaP cells. (B) Western blot analysis was performed on whole protein lysates from untreated PC-3, DU145 and LNCaP cells with specific $\beta 1$ integrin antibody. $\alpha$-tubulin served as a protein loading control. The highly invasive and androgen-independent cell lines PC-3 and DU145 showed the highest $\beta 1$ integrin expression, whereas the non-invasive and androgen-dependent LNCaP cells showed only a moderate expression of $\beta 1$ integrin.

\subsubsection{Leupaxin influences ITGB1 expression}

To further investigate the influence of LPXN on ITGB1 expression, LPXN expression was downregulated in PC-3 and DU145 cells by RNA interference. Protein and RNA was isolated 48 hours after transfection. Luciferase siRNA transfected cells served as a control. Quantitative real time PCR (qRT-PCR) was performed using specific primer pairs for LPXN (LPXN-CDS-F1 and LPXN-CDS-R1) and ITGB1 (ITGB1-Q2-Fw and ITGB1Q2-Rev), respectively. A reduced expression of ITGB1 in PC-3 and DU145 cells was shown (Fig. 3.12A). This reduction of ITGB1 expression was also observed on the protein level (Fig. 3.13B). 

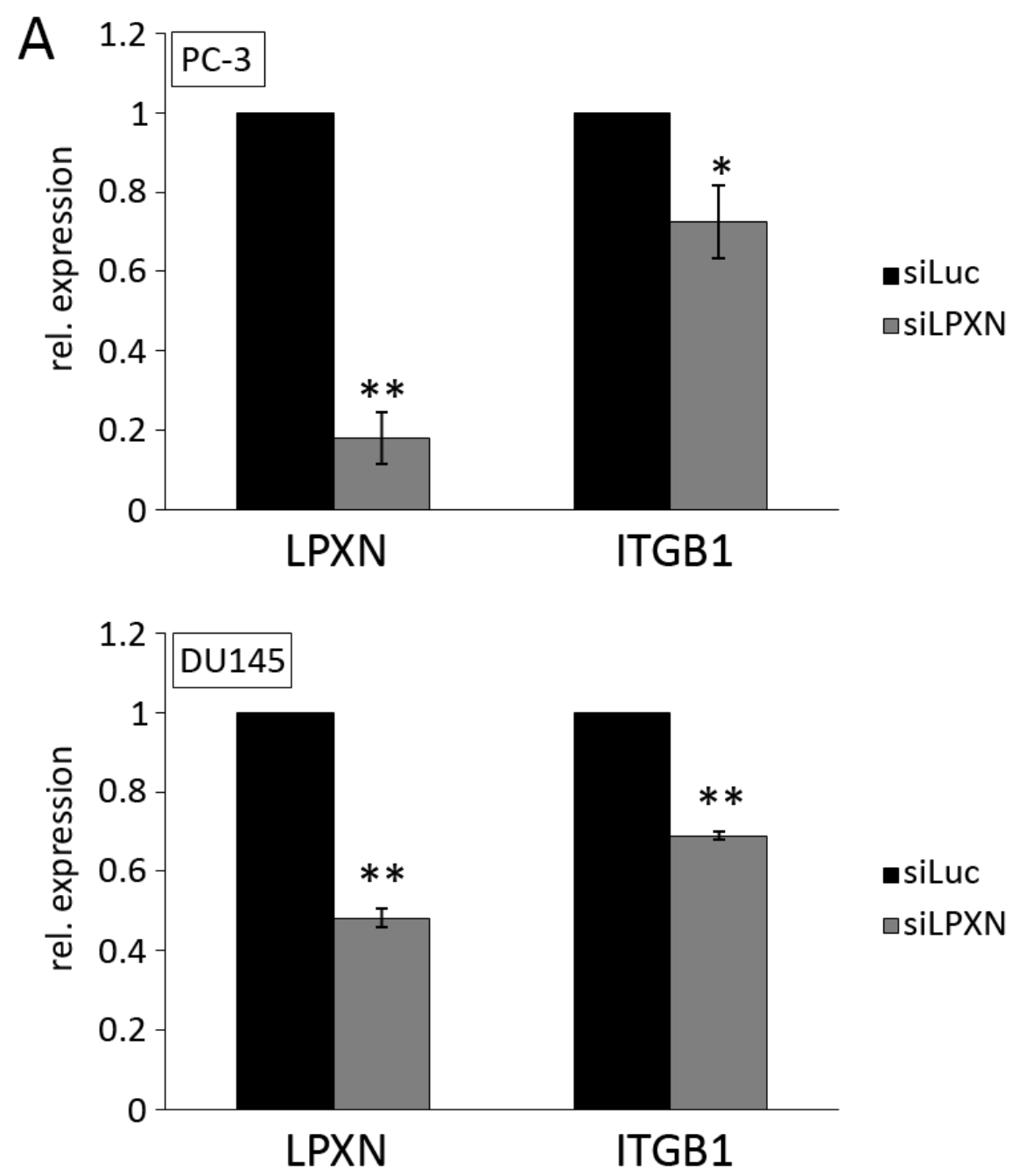

B

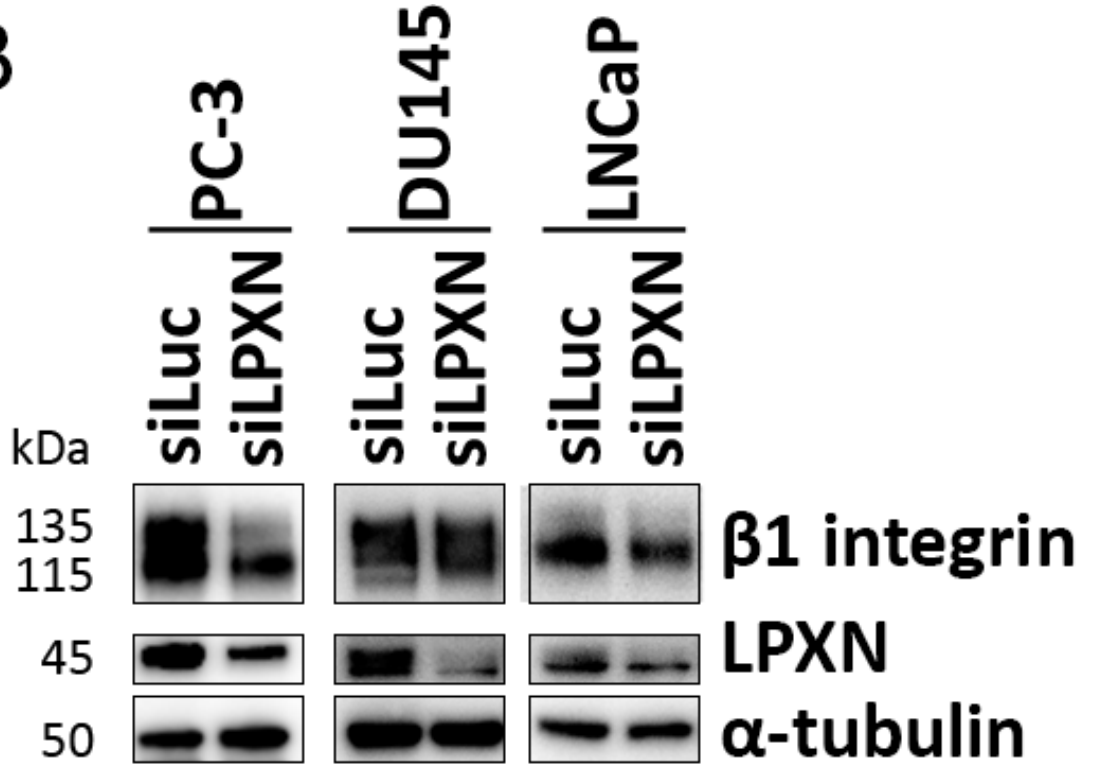

Fig. 3.12: Integrin $\beta 1$ expression after down regulation of LPXN expression. (A) To analyze ITGB1 expression after LPXN knockdown, PC-3 and DU145 cells were transfected with siRNA against the LPXN gene (siLPXN) and the luciferase gene as a control (siLuc). 48 hours after transfection total RNA was 
isolated and subsequently subjected to qRT-PCR using the specific primer pairs LPXN-CDS-F1 and LPXNCDS-R1 as well as ITGB1-Q2-Fw and ITGB1-Q2-Rev. ITGB1 expression is reduced after LPXN knockdown in all PCa cell lines. The illustrated values represent an average of three independent experiments, which were measured in duplicate. ${ }^{*}$ are indicating statistical significance as judged by students $t$-test $(p \leq 0.05$ $\left({ }^{*}\right) ; p \leq 0.001\left(^{* *}\right) ; p \leq 0.0001\left(^{* * *}\right) ; n . s .=$ not significant. (B) Western blot analysis of the PCa cell lines PC-3, DU145 and LNCaP showing reduced $\beta 1$ integrin expression after LPXN knockdown. All cell lines showed decreased levels of $\beta 1$ integrin after LPXN knockdown, with the most prominent effect in PC-3 cells. This down regulation of $\beta 1$ integrin was not observed in control siLuc-transfected cells. $\alpha$-tubulin served as a loading control.

Likewise, overexpression of an EGFP-LPXN fusion construct caused a slight increase of ITGB1 expression in DU145 cells in comparison to EGFP control transfected cells. In PC3 cells the EGFP-LPXN induced ITGB1 expression was not detectable (Fig. 3.13). Although the effect of EGFP-LPXN overexpression on ITBG1 expression was not very strong, there is a significant correlation between LPXN and ITGB1 expression. 


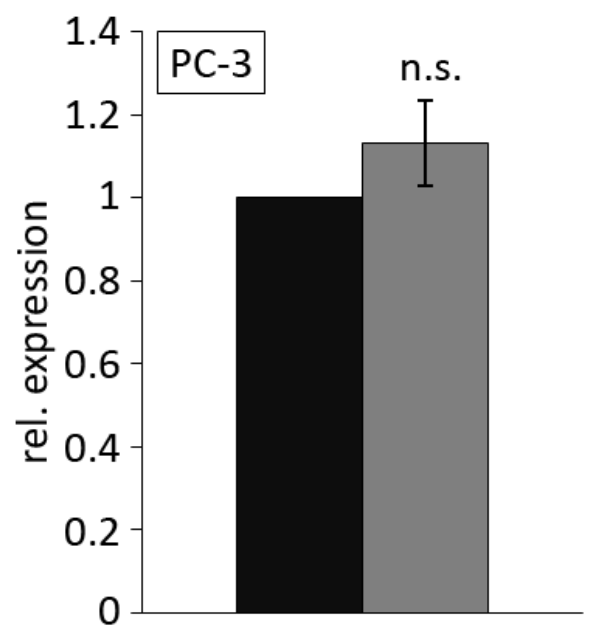

ITGB1

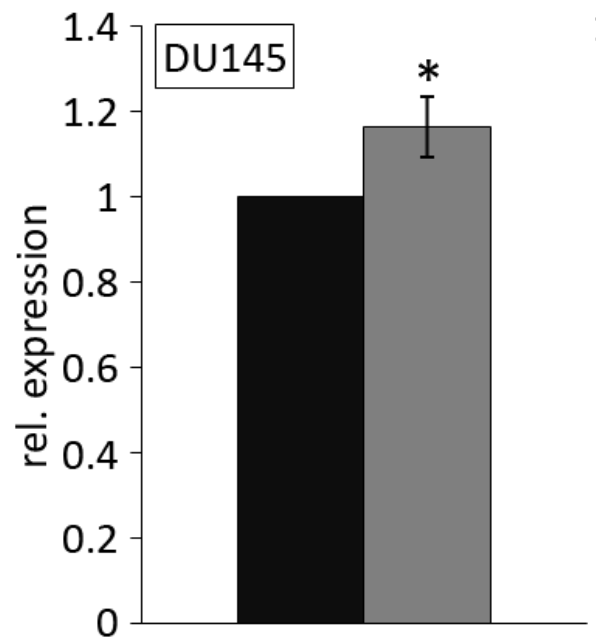

ITGB1

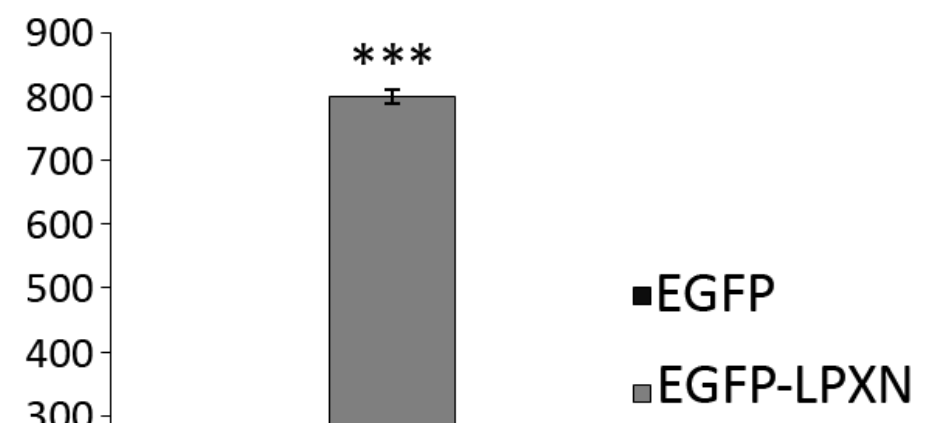

LPXN

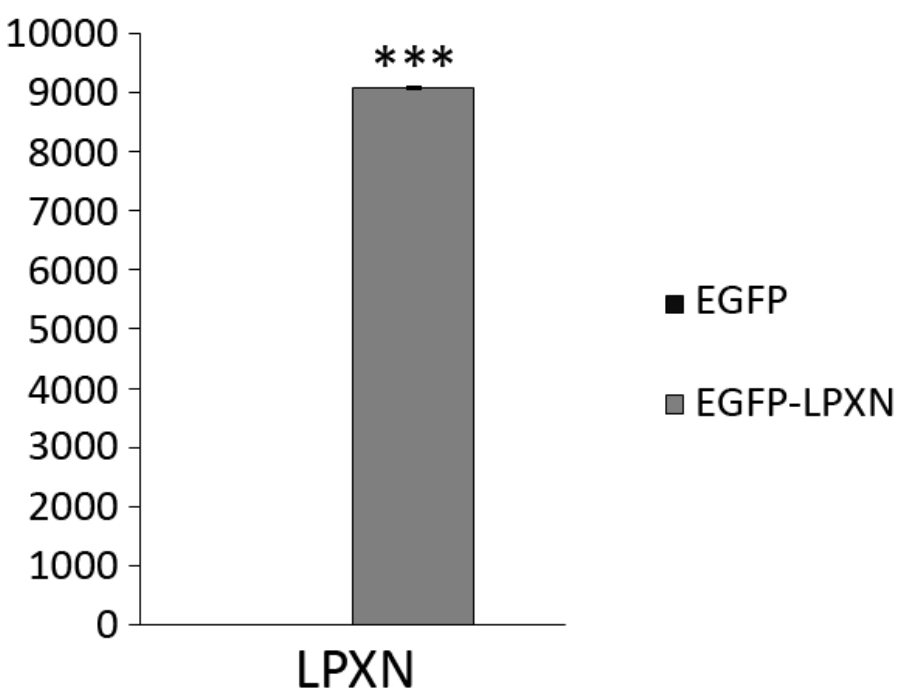

Fig. 3.13: $\beta 1$ integrin expression after EGFP-LPXN overexpression in PCa cell lines. The indicated PCa cells were transfected with an EGFP-LPXN fusion construct and with EGFP alone as a control. To analyze correlation of LPXN and ITGB1 expression RNA was isolated 48 hours after transfection, reverse transcribed and subjected to qRT-PCR. LPXN CDS F1R1 primer confirmed successful transfection and overexpression of EGFP-LPXN. DU145 showed a significant 1.2-fold upregulation of ITGB1 after EGFPLPXN overexpression, which was not present in PC-3. Stars indicate statistical significance as calculated by students t-test $\left({ }^{*}=0.01 \leq \mathrm{p} \leq 0.05 ;{ }^{* *}=0.001=\mathrm{p} \leq 0.01 ;{ }^{* * *}=0.0001 \leq \mathrm{p} \leq 0.001 ;\right.$ n.s. $=$ not significant $)$

Western blot experiments also showed an upregulation of ITGB1 expression in PC-3 and DU145 cells upon EGFP-LPXN transfection in comparison to EGFP control transfected cells. This induction of ITGB1 expression was not present in LNCaP cells (Fig. 3.14). Thus, the LPXN-mediated effects on ITGB1 expression might account for the reduced adhesion of PCa cells after LPXN knockdown. 


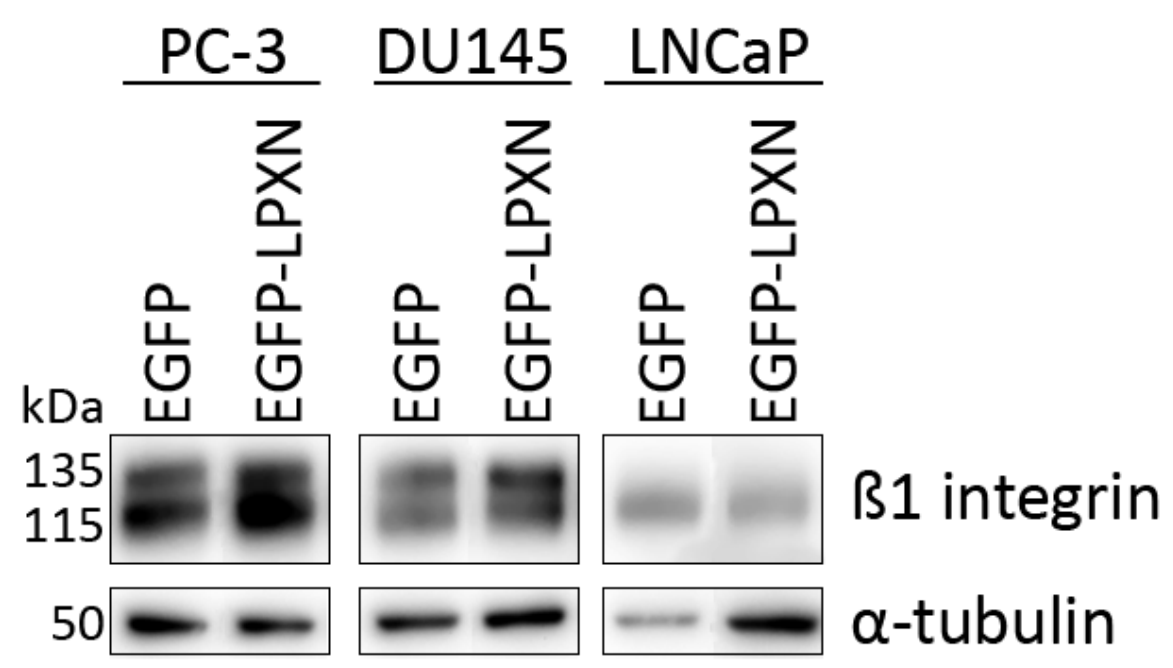

Fig. 3.14: Overexpression of LPXN results in increased $\beta 1$ integrin expression. To analyze the effect of LPXN overexpression on $\beta 1$ integrin, the PCa cell lines PC-3, DU145 and LNCaP were transfected with an EGFP-LPXN construct or EGFP as a control, respectively. 24 hours after transfection whole protein was isolated and subjected to western blot analyses. Specific detection of $\beta 1$ integrin showed elevated levels of $\beta 1$ integrin expression after LPXN overexpression. This effect was most prominent in PC-3 and DU145 cells. $\alpha$-tubulin served as a protein loading control.

\subsubsection{LPXN influences activity of the specificity protein 1 (Sp1)}

From the literature it is known that integrins, especially $\alpha 6$ integrin harbor binding sites for the transcription factor specificity protein 1 (Sp1) in their promoter region and that Sp1 is able to positively influence $\alpha 6$ promoter activity (Gaudreault et al. 2007). We therefore tested whether LPXN can influence the transcriptional activity of Sp1 by a reporter gene assay. This assay contains a firefly luciferase gene under the control of a minimal CMV promoter and tandem repeats of the Sp1 transcriptional response element (TRE). On the one hand PC-3, DU145 and LNCaP cells were plated on 96-well plates and double transfected with either pEGFP-C1-LPXN or pEGFP-C1 and the Cignal ${ }^{\mathrm{TM}}$ reporter plasmid. On the other hand, cells were transfected with either siLPXN or siLuc siRNA. After 24 hours the Cignal $^{\text {TM }}$ reporter plasmid was transfected separately. Measurement of the luciferase activity revealed that overexpression of LPXN induced a significant increase in Sp1 activity in DU145 and LNCaP cells. This increase was also visible in PC-3, 
but not statistically significant. On the other hand, knockdown of LPXN did also display increased Sp1 activity, which was not statistically significant for all PCa cell lines (Fig. 3.15).
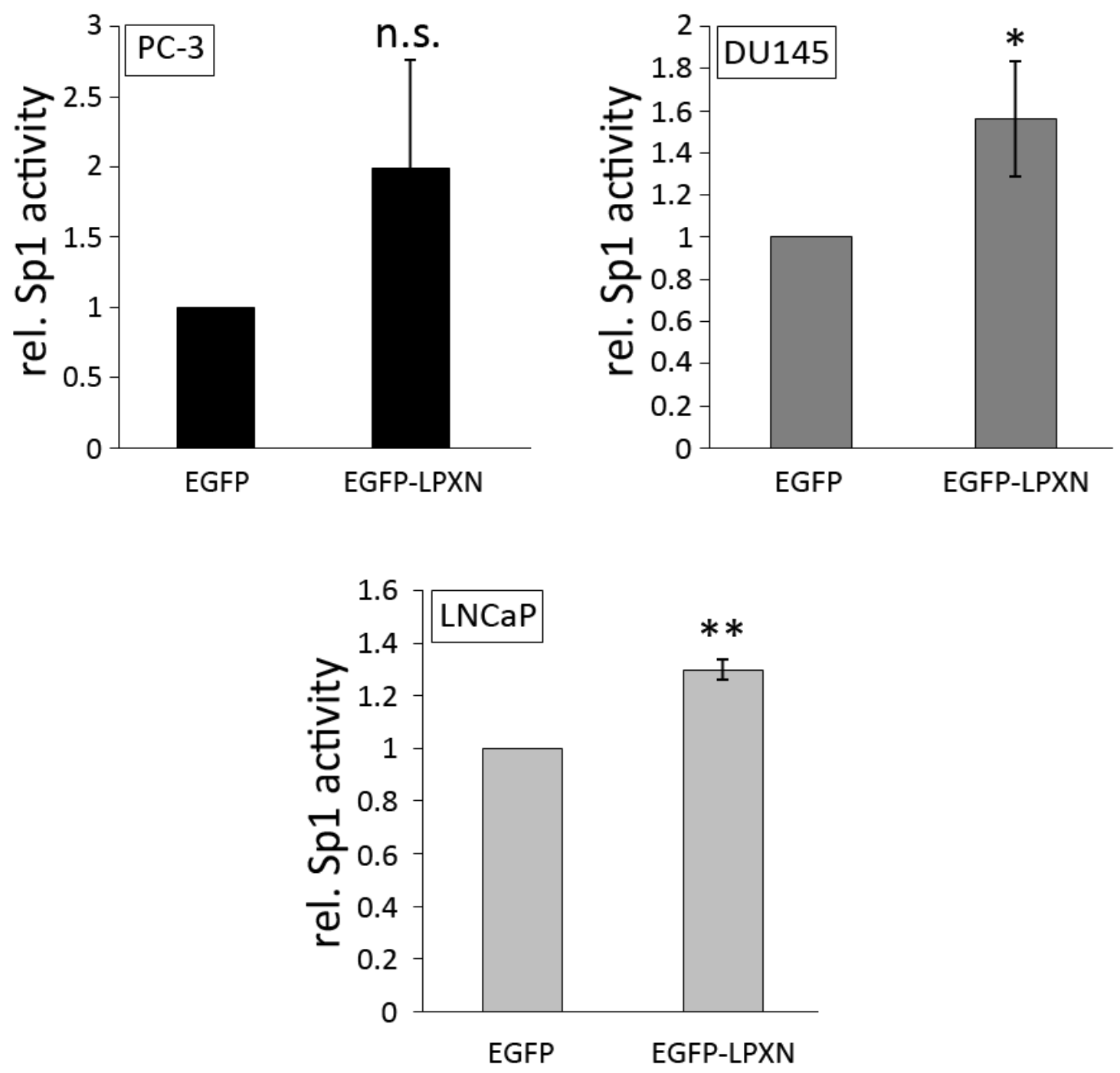

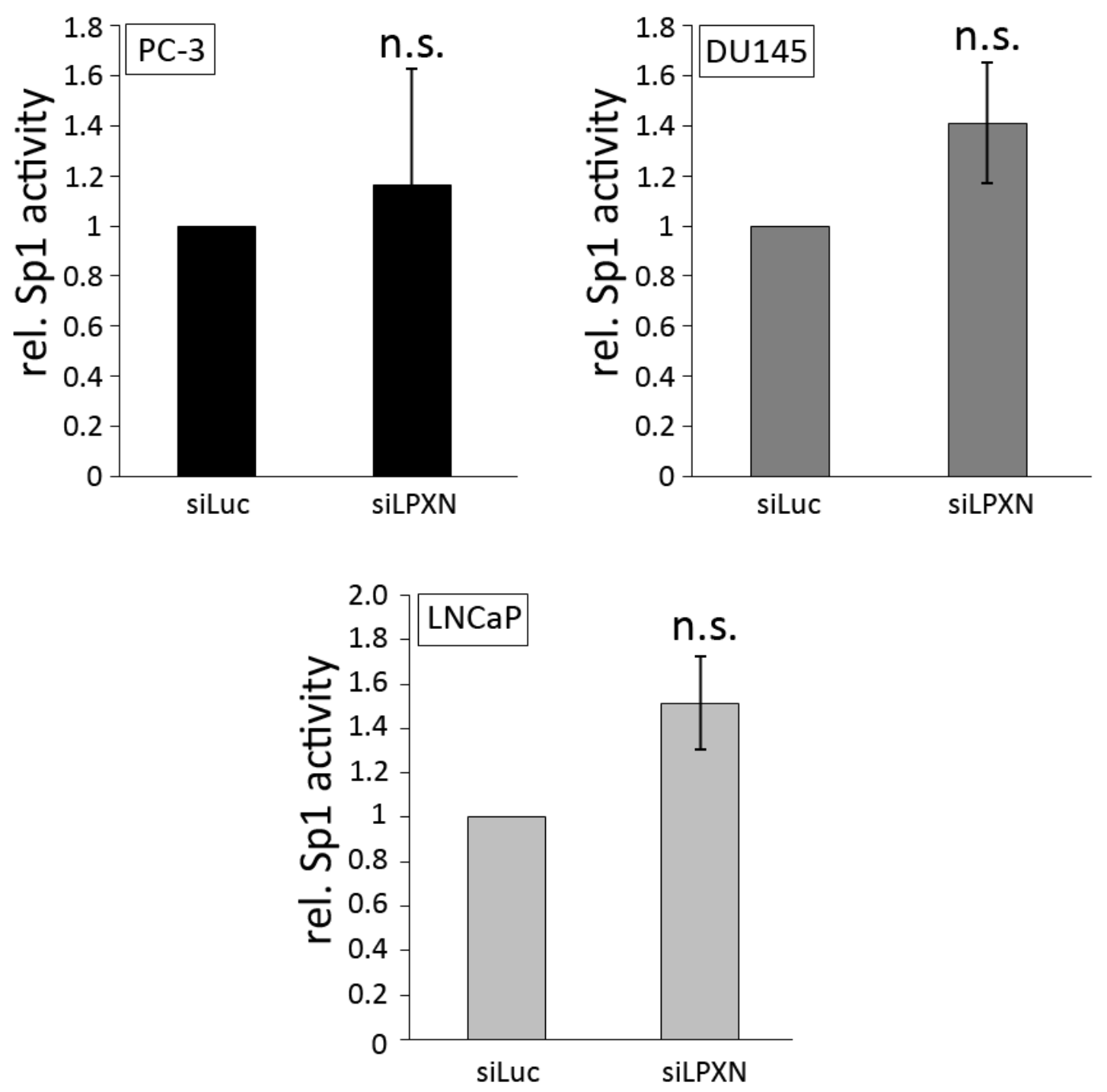

Fig. 3.15 Sp1 activity in PC-3, DU145 and LNCaP cells after modulation of LPXN expression. To analyze Sp1 activity the Cignal ${ }^{\mathrm{TM}}$ Sp1 activity assay was used. PC-3, DU145 and LNCaP cells were plated on 96-well plates and transfected with a pEGFP-LPXN fusion construct and the reporter plasmid (Cignal ${ }^{\mathrm{TM}}$ ) to check the effect of LPXN overexpression on Sp1 activity. Likewise, for LPXN knockdown, the cells were plated on 96-well plates and transfected with siRNA against the LPXN and luciferase gene, respectively. On the next day, the cells were transfected with the reporter plasmid. Sp1 activity was determined by measurement of luciferase activity 24 hours after transfection. Values displayed are normalized to siLuc or EGFP, respectively and represent relative values averaged from triplicates from three independent experiments. Stars indicate statistical significance as calculated by students $t$-test $\left({ }^{*}=0.01 \leq p \leq 0.05\right.$; ${ }^{* *}=0.001=\mathrm{p} \leq 0.01{ }^{* * *}=0.0001 \leq \mathrm{p} \leq 0.001 ;$ n.s. $=$ not significant $)$ 


\subsection{LPXN affects radio-resistance of PCa cells}

Cellular adhesion is a process that is also implicated in the acquisition of radio- and chemo-resistances of several cancer entities. It was already shown for head and neck cancer that targeting the $\beta 1$ integrin is beneficial for tumor cell radiotherapy (Eke et al. 2012). Integrins are a major mediator of cellular adhesion and their deregulation is a common feature of the aggressive and metastatic phenotype in several types of cancer, including PCa.

To analyze if the ITGB1-regulating effects that LPXN exerts on cell adhesion do also affect the resistance to ionizing radiation, PC-3, DU145 and LNCaP cells were transfected with siRNA against LPXN, whereas siRNA against the luciferase gene (Luc) served as a control. After transfection a defined cell number was plated on to 6-well plates and exposed to single dose ionizing irradiation of $0,2,4$ and $8 \mathrm{~Gy}$, respectively, on the next day. Subsequently, the clonogenic cell survival was evaluated by a colony formation assay. The assay clearly showed a sensitization of PCa cells against ionizing radiation after LPXN knockdown. With increasing irradiation intensity this effect was significantly detectable in all cell lines. LNCaP cells showed the highest sensitivity to irradiation, e.g. after a single dose of 2 Gy the sensitizing effect of LPXN knockdown was already visible. However, DU145 and PC-3 cells were quite resistant to low doses of irradiation and started to show significant sensitizing effects from doses of 4 Gy on (Fig. 3.16). 


\section{PC-3}

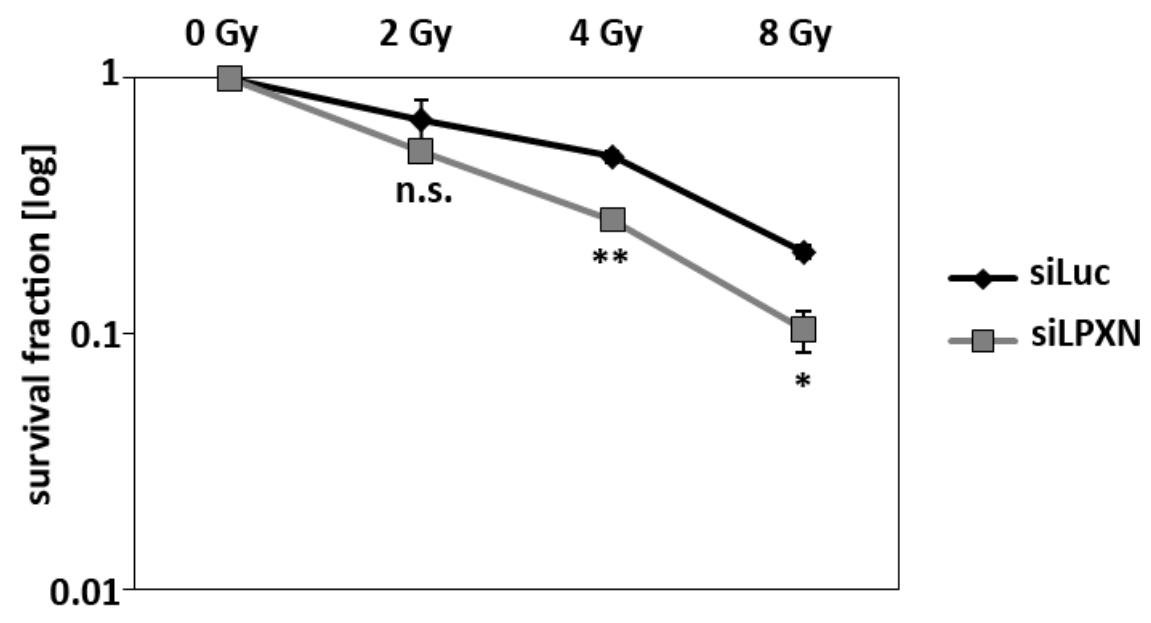

\section{DU145}
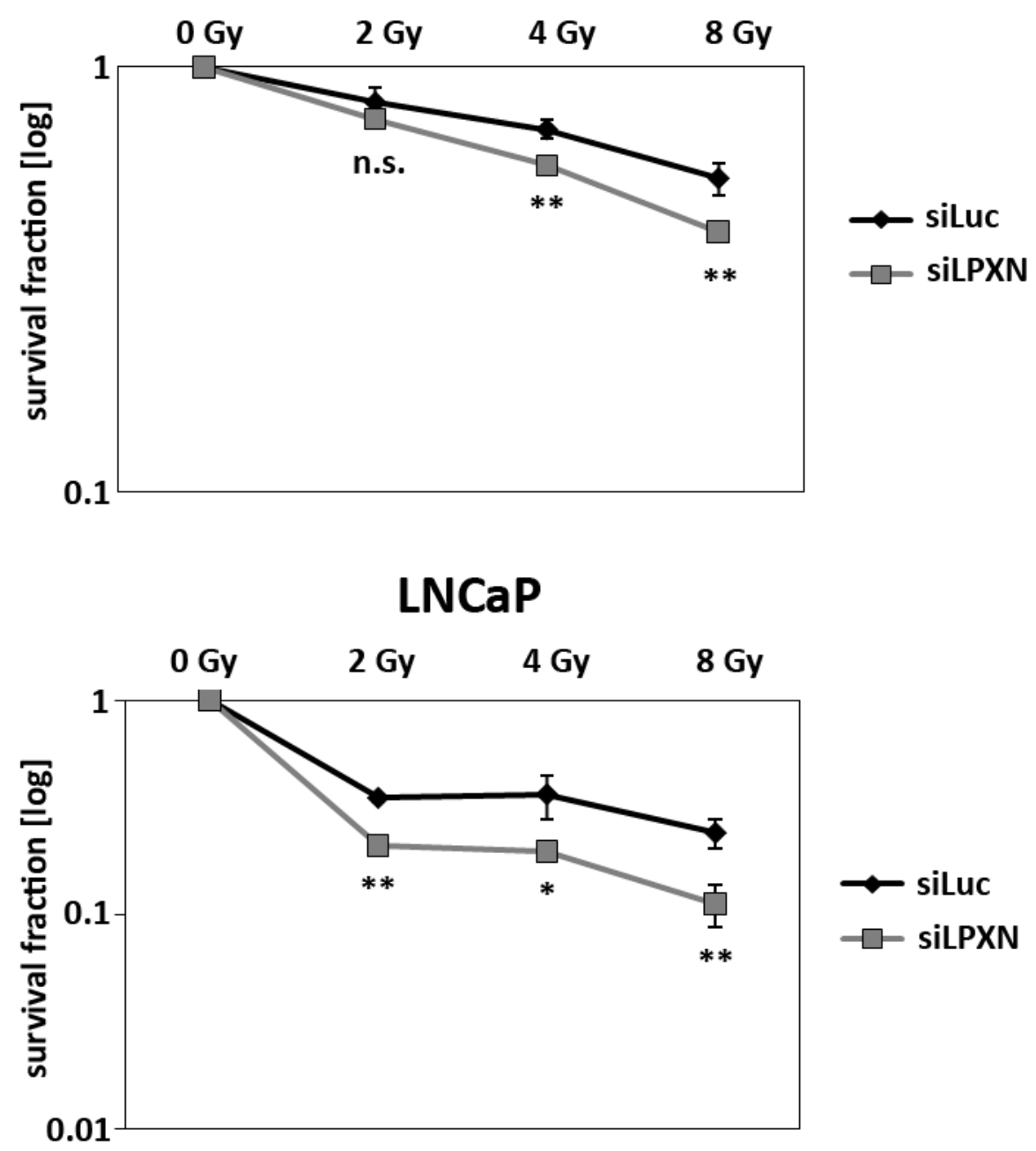

Fig. 3.16: Response of PCa cells to ionizing radiation after downregulation of LPXN expression. PC-3, DU145 and LNCaP cells were transfected with siRNA against the LPXN (siLPXN) or luciferase (siLuc) gene. 
After transfection a defined number of cells was plated on 6-well plates and exposed to single dose ionizing irradiation of $0,2,4$, and 8 Gy on the next day. Cells were incubated until colonies formed and colonies were stained and counted. Survival fraction was calculated. Data represents means \pm s.d. from three experiments. All PCa cell lines showed a significant radio-sensitization upon knockdown of LPXN expression, which was highest in the LNCaP cell line. Stars represent statistical significance as calculated by students t-test $\left({ }^{*}=0.01 \leq \mathrm{p} \leq 0.05 ;{ }^{* *}=0.001=\mathrm{p} \leq 0.01 ;{ }^{* * *}=0.0001 \leq \mathrm{p} \leq 0.001\right)$.

Next we assessed if inhibition of $\beta 1$ integrin is able to show the same effect upon irradiation that we observed for LPXN knockdown. Since Cordes et al. already described sensitization to irradiation by the inhibition of $\beta 1$ integrin in head and neck squamous cell carcinoma cells and proposed that $\beta 1$ integrin could be used as a molecular target in combination with radiotherapy to sensitize tumor cells and increase survival of patients (Cordes, Park 2007), we used the inhibitory monoclonal anti- $\beta 1$ integrin antibody (AIIB2, kindly provided by Prof. Dr. med. Nils Cordes, Oncoray, Dresden, Germany) to block $\beta 1$ integrin activity of our PCa cell lines PC-3, DU145 and LNCaP. To test clonogenic cell survival a defined number of cells was plated on 6-well plates. One hour before the cells were exposed to different doses of irradiation (0, 2, 4, 8 Gy), the inhibitory anti- $\beta 1$ antibody as well as a control rat IgG were added to the growth medium at a final concentration of $1 \mu \mathrm{g} / \mathrm{ml}$. Clonogenic cell survival was evaluated and revealed that also inhibition of $\beta 1$ integrin significantly sensitized PCa cells to ionizing irradiation. As it was observed for sensitization by LPXN knockdown, LNCaP cells showed the highest amount of sensitization by AIIB2-mediated $\beta 1$ integrin inhibition. Whereas, PC-3 and DU145 cells were quite resistant to low doses of irradiation and the sensitizing effect of AIIB2 to irradiation was only observed at doses higher than 4 Gy (Fig. 3.17). 


\section{PC-3}

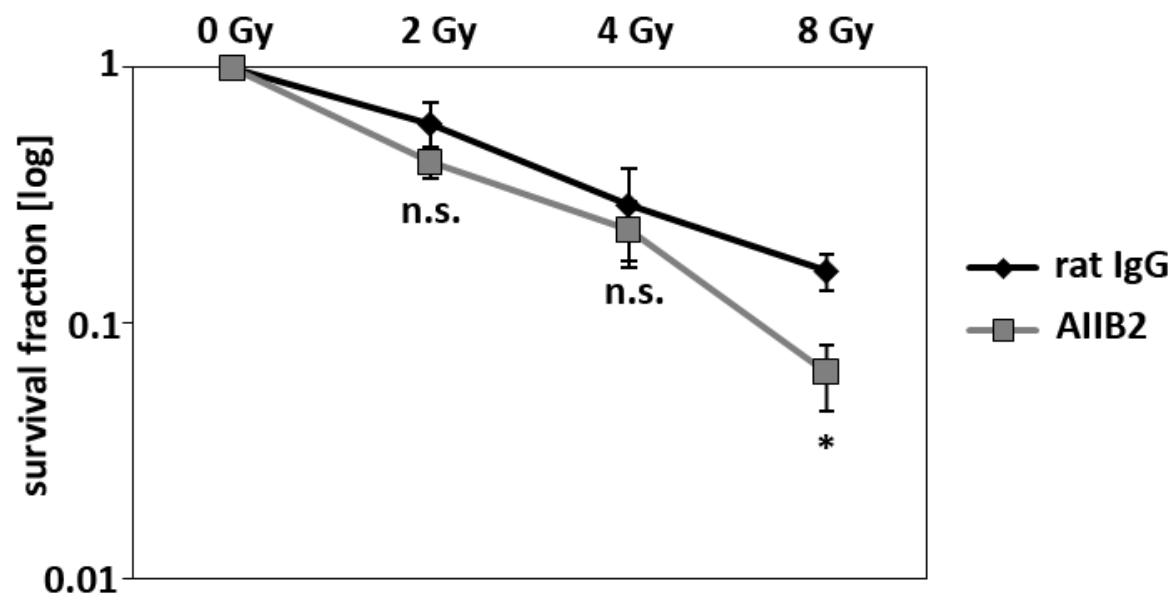

\section{DU145}

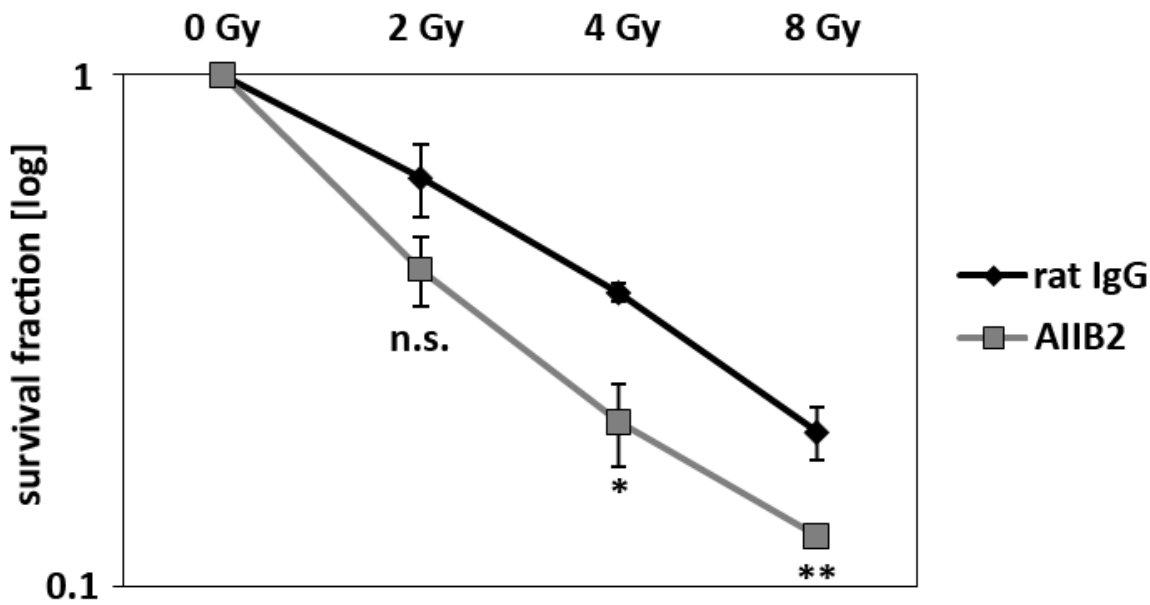

LNCaP

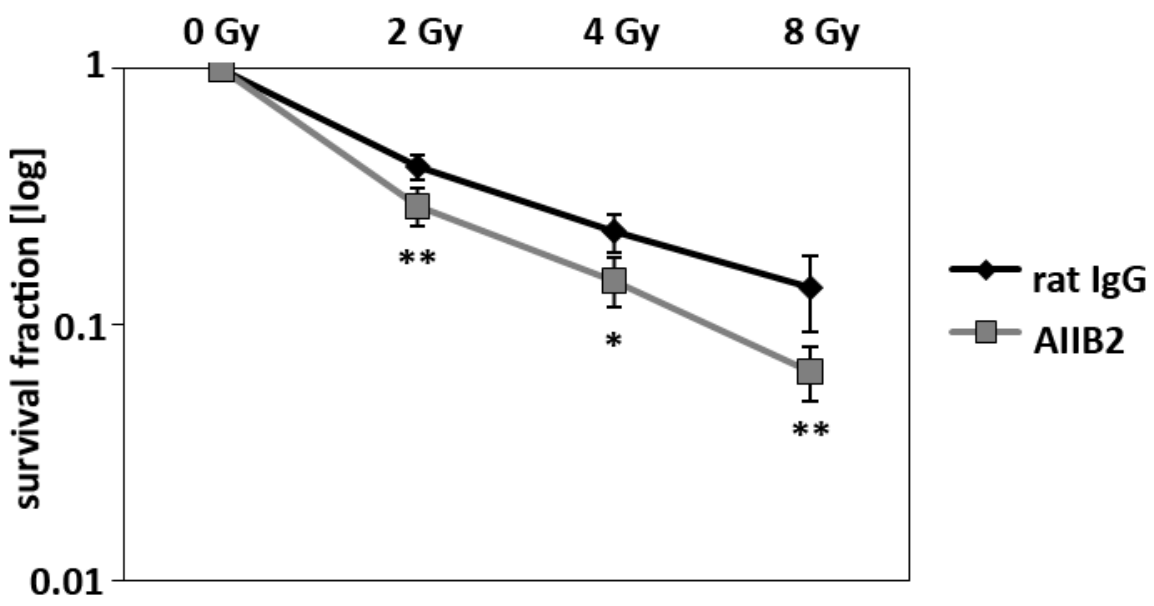

Fig. 3.17: Radio-sensitization of PC-3, DU145 and LNCaP cells after inhibition of $\beta 1$ integrin. To evaluate clonogenic cell survival after $\beta 1$ integrin inhibition, PC-3, DU145 and LNCaP cells were plated on 6-well plates at a defined cell number. One hour before cells were exposed to different doses of irradiation 
$(0,2,4,8 \mathrm{~Gy})$ the inhibitory anti- $\beta 1$ integrin antibody (AIIB2), as well as a control rat IgG were applied to the growth medium of the cells at a final concentration of $1 \mu \mathrm{g} / \mathrm{ml}$. Colony formation assay was performed as described in Material and Methods. Cell colonies were stained, counted and survival fraction was calculated. The inhibition of the $\beta 1$ integrin significantly sensitized the PCa cells to irradiation. Data represent means \pm s.d. from three experiments. Stars represent statistical significance as calculated by students t-test $\left({ }^{*}=0.01 \leq \mathrm{p} \leq 0.05 ;{ }^{* *}=0.001=\mathrm{p} \leq 0.01 ;{ }^{* * *}=0.0001 \leq \mathrm{p} \leq 0.001\right)$.

Having shown a sensitization of PCa cells to ionizing irradiation after LPXN knockdown or $\beta 1$ integrin blockade, we wondered if both treatments might have a synergistic effect and could even enhance sensitization to ionizing irradiation.

Therefore, PCa cells were transfected with siRNA against the LPXN and luciferase gene, respectively. After 24 hours, the cells were plated on a 6-well plate at a defined cell number. On the next day, one hour before irradiation cells were treated with the inhibitory AIIB2 antibody at a final concentration of $1 \mu \mathrm{g} / \mathrm{ml}$. Analyses of the corresponding colony formation assays revealed that the LPXN-induced radiosensitization of PC-3 and DU145 cells could not be further enhanced by the blockade of $\beta 1$ integrin (Fig. 3.18). Thus, these results show that simultaneous knockdown of LPXN and $\beta 1$ integrin blockade do not have a synergistic effect on radio-sensitization of PCa cells. Furthermore, this result indicates that LPXN might influence radio-resistance mechanisms by regulation of ITGB1 expression. 


\section{PC-3}

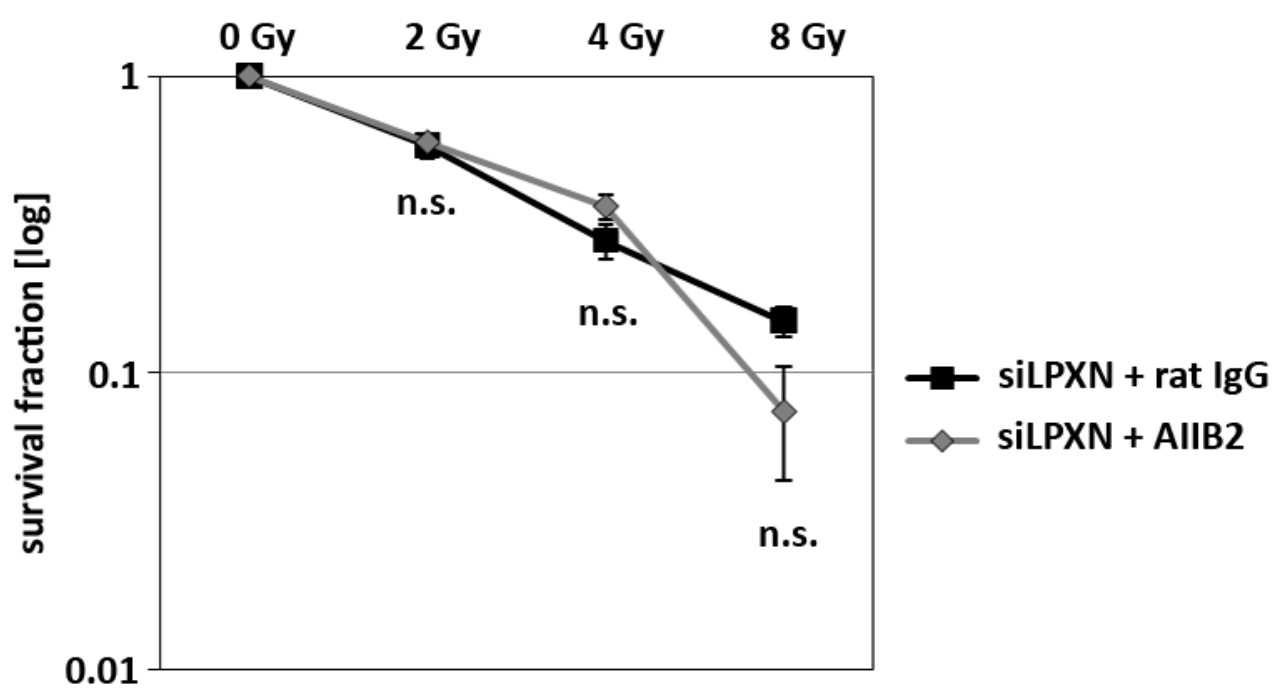

DU145

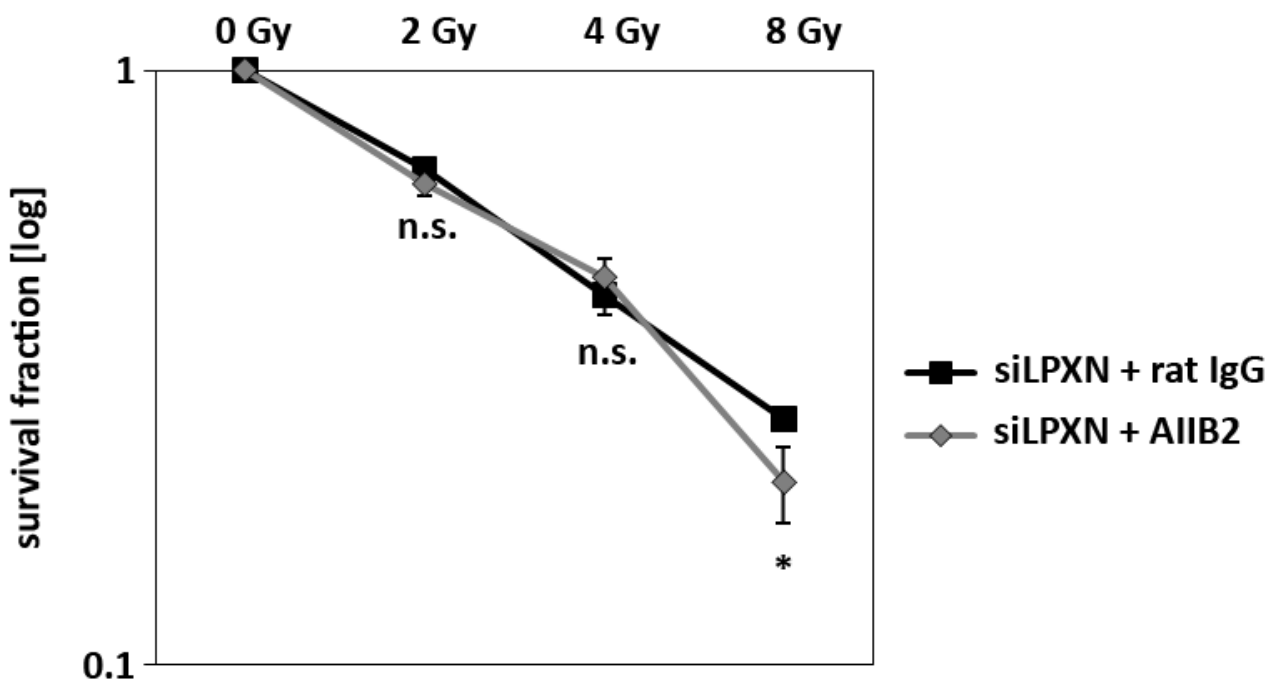

Fig. 3.18: Colony formation assay of irradiated PCa cells after simultaneous knockdown of LPXN and $\beta 1$ integrin blockade. Clonogenic cell survival of PC-3 and DU145 was evaluated to determine a synergistic effect of simultaneous downregulation of LPXN and blockade of $\beta 1$ integrin on radiosensitization of PCa cells. Cells transfected with siRNA against the LPXN gene were plated on 6-well plates at a defined cell number. One hour before cells were exposed to different doses of irradiation (0, 2, 4, 8 Gy) the inhibitory anti- $\beta 1$ integrin antibody (AIIB2) were applied to the growth medium of the cells at a final concentration of $1 \mu \mathrm{g} / \mathrm{ml}$. Colony formation assay was performed as described in Material and Methods. Cell colonies were stained, counted and survival fraction was calculated. Simultaneous knockdown of LPXN and blockade of $\beta 1$ integrin do not have a synergistic effect on radio-sensitization of PCa cells. Data represent means \pm s.d. from three experiments. Stars represent statistical significance as calculated by students t-test $\left({ }^{*}=0.01 \leq \mathrm{p} \leq 0.05 ;{ }^{* *}=0.001=\mathrm{p} \leq 0.01 ;{ }^{* * *}=0.0001 \leq \mathrm{p} \leq 0.001 ; \mathrm{n} . \mathrm{s} .=\right.$ not significant $)$. 


\subsubsection{LPXN expression is enhanced upon ionizing radiation}

As we were able to sensitize PCa cells to radiation by downregulation of LPXN expression, LPXN might be involved in these resistance mechanisms. In order to test if the cells rely on LPXN expression to acquire radio-resistance we exposed PC-3, DU145 and LNCaP cells to a single dose irradiation of $4 \mathrm{~Gy}$. Total RNA was isolated 1, 2, 24 and 48 hours after irradiation. Un-irradiated cells were taken as a control (0h). The following qRT-PCR analysis demonstrated that during the early time points of 1 and 2 hours after irradiation a decrease in LPXN expression for PC-3, DU145 and LNCaP cells was observed, whereas the expression of LPXN increased after 24 and 48 hours (Fig. 3.19).
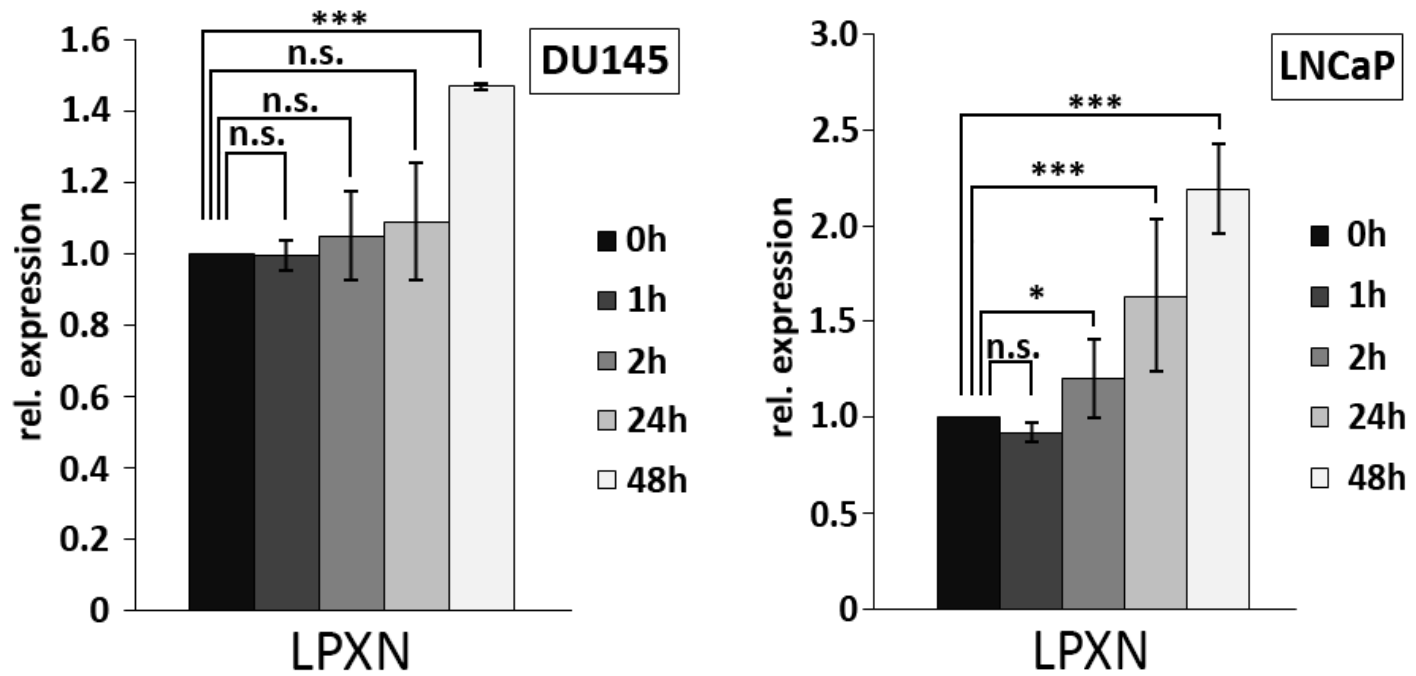

Fig. 3.19: Irradiation-induced expression of LPXN in DU145 and LNCaP cells. DU145 and LNCaP cells were plated on 6-well plates to analyze irradiation-induced expression of LPXN.Total RNA was isolated 1, 2,24 , and 48 hours after exposure to a single dose irradiation of $4 \mathrm{~Gy}$, reverse transcribed and subjected to qRT-PCR. Un-irradiated cells $(0 \mathrm{~h})$ were used as a control. LPXN-specific primers showed an increasing LPXN expression after single dose irradiation in DU145 and LNCaP cells. The illustrated values represent an average of three independent experiments, which were measured in duplicate and normalized to the LPXN expression in control cells $(0 \mathrm{~h})$. Stars indicate statistical significance as judged by students t-test $\left(\mathrm{p} \leq 0.05\left(^{*}\right) ; \mathrm{p} \leq 0.001\left(^{* *}\right): \mathrm{p} \leq 0.0001\left(^{* * *}\right)\right.$. 


\subsubsection{ITGB1 expression is enhanced upon ionizing radiation}

In order to analyze ITGB1 expression after ionizing radiation, DU145 and LNCaP cells were exposed to a single dose irradiation of $4 \mathrm{~Gy}$. Total RNA and protein was isolated at $0,1,2,24$ and 48 hours after irradiation, whereas cells from 0 hours time point were not irradiated and served as a control. Total RNA was isolated, reverse transcribed and subjected to qRT-PCR. Subsequent analysis revealed that directly after irradiation ITGB1 expression decreased in DU145, but started to rise again already two hours after irradiation. Although this increase is not statistically significant in DU145 cells, there is a clear trend noticeable. In LNCaP cells expression levels of ITGB1 did not decline after irradiation but were steadily increasing and significantly upregulated 1.4-fold at 24 and 1.7-fold at 48 hours after exposure to single dose irradiation (Fig. 3.20).

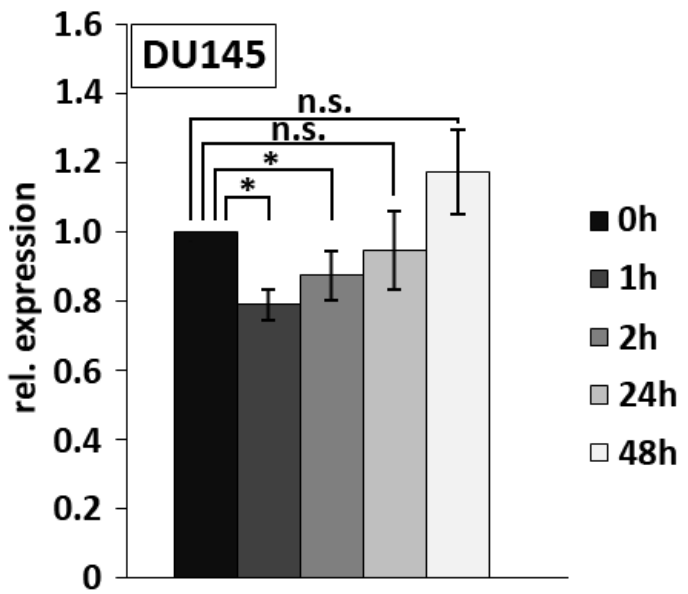

ITGB1

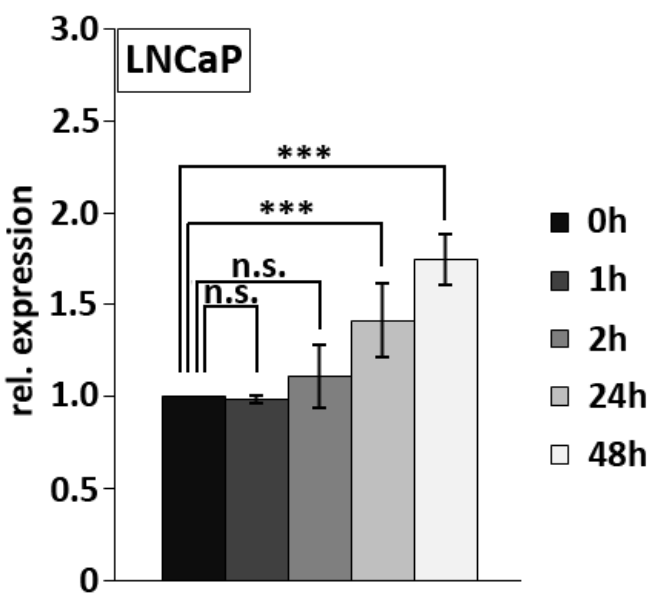

ITGB1

Fig. 3.20: Irradiation-induced upregulation of ITGB1 in DU145 and LNCaP cells. DU145 and LNCaP cells were plated on 6-well plates to analyze irradiation-induced expression of ITGB1. Cells were exposed to a single dose irradiation of 4 Gy and total RNA was isolated at 1, 2, 24, and 48 hours after irradiation, reverse transcribed and subjected to qRT-PCR. Un-irradiated cells (0h) were used as a control. The qRTPCR analysis using ITGB1-specific primers showed that 24 hours after irradiation ITGB1 expression is significantly increased in LNCaP cells (1.4-fold), whereas in DU145 ITGB1 expression declines immediately after irradiation and steadily rises until it reaches a peak at 48 hours (1.2-fold). The illustrated values represent an average of three independent experiments, which were measured in duplicate and normalized to the ITGB1 expression in control cells (0h). Stars are indicating statistical significance as judged by students t-test $\left(\mathrm{p} \leq 0.05\left(^{*}\right) ; \mathrm{p} \leq 0.001\left(^{* *}\right): \mathrm{p} \leq 0.0001\left(^{* * *}\right)\right.$. 
Western blot analysis also showed an increased $\beta 1$ integrin expression over time with a peak at 48 hours in DU145 and LNCaP cell lines. $\alpha$-tubulin bands indicate equal protein loading (Fig. 3.21).

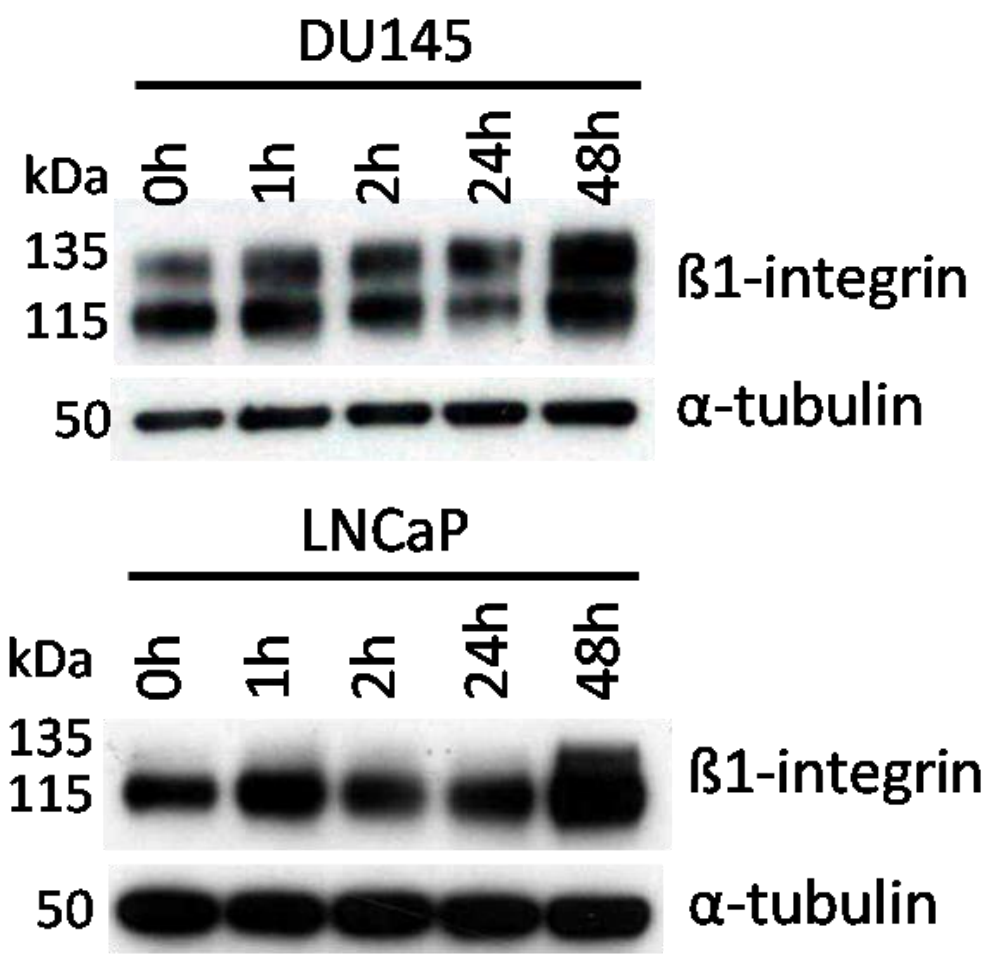

Fig. 3.21: Expression of $\beta 1$ integrin in DU145 and LNCaP at different time points after irradiation. DU145 and LNCaP cells were plated on 6-well plates to analyze irradiation-induced expression of ITGB1. Cells were exposed to a single dose irradiation of 4 Gy and total protein was isolated at 1, 2, 24, and 48 hours after irradiation and subjected to western blot analysis. Non-irradiated cells (0h) were used as a control. Western blot analysis was performed using a $\beta 1$ integrin-specific antibody. $\alpha$-tubulin ensured equal protein loading. The PCa cell lines showed an upregulation of ITGB1 expression with proceeding time and a peak at 48 hours after irradiation.

\subsubsection{LPXN knockdown prevents radiation-induced upregulation of ITGB1 expression}

Having shown a clear correlation of LPXN and ITGB1 expression we were wondering if knockdown of LPXN could prevent radiation-induced upregulation of ITGB1. Therefore, LPXN expression was downregulated in PC-3 and DU145 cells 48 hours before they were exposed to a single dose irradiation of $4 \mathrm{~Gy}$. Total RNA was isolated at 1, 2, 24, and 
48 hours after irradiation. Cells transfected with siRNA against the luciferase gene (siLuc) and non-irradiated cells (0h) served as a control. qRT-PCR of control transfected cells (siLuc) showed radiation-dependent increases of ITGB1 expression. Reduction of LPXN expression by RNA interference (siLPXN) could prevent this radiation-induced upregulation of ITGB1 even after 48 hours. Compared to siLuc-transfected cells, which showed the characteristic increases in ITGB1 expression levels, ITGB1 stayed at a quite equal expression level of approximately $60 \%$ in siLPXN-transfected cells over the time course of the experiment (Fig. 3.22). This indicates an involvement of LPXN in ITGB1 regulation and points towards a putative mechanism of LPXN-induced radio-resistance of PCa cells.

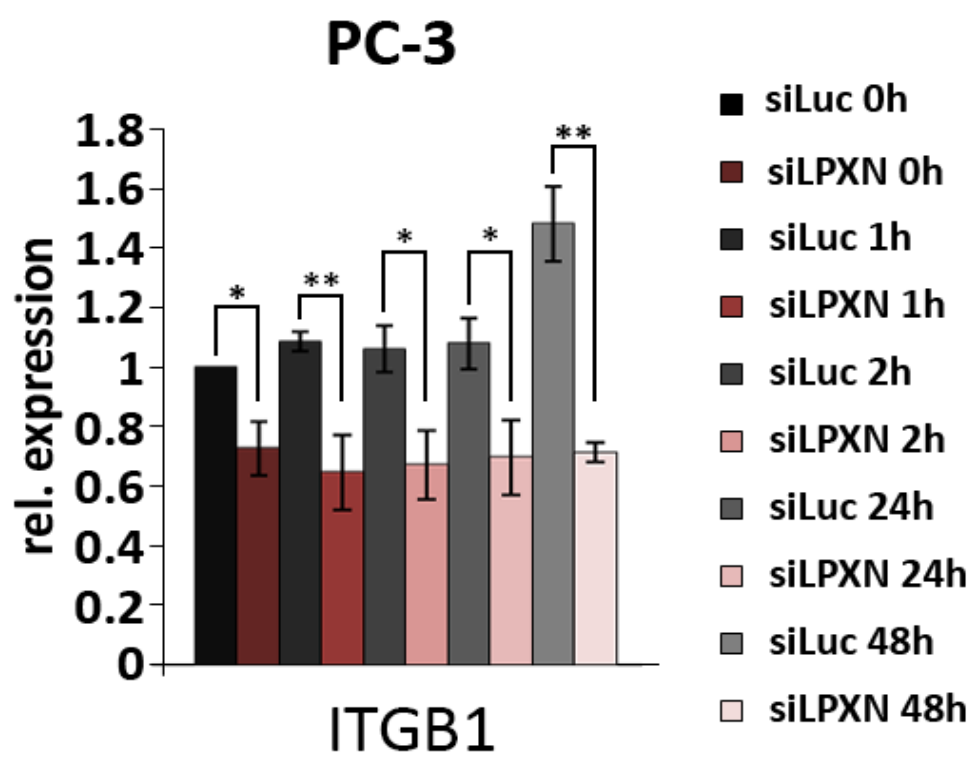

\section{DU145}

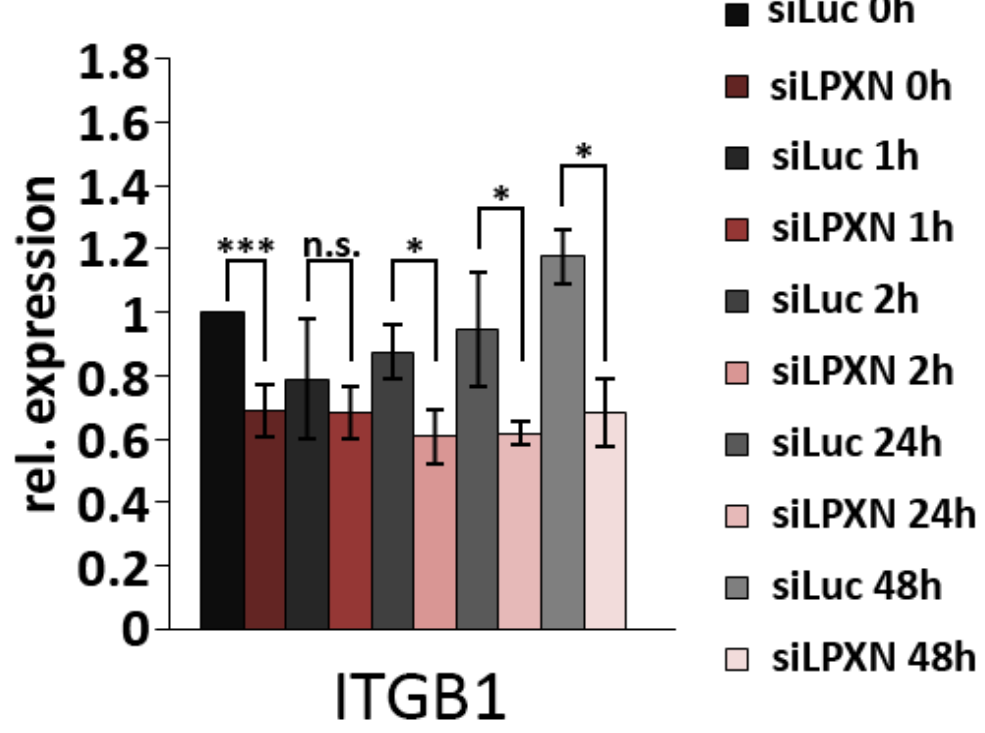


Fig. 3.22: LPXN knockdown in PC-3 and DU145 cells prevents irradiation-induced upregulation of

ITGB1. PC-3 and DU145 cells were plated on 6-well plates and transfected with siRNA against the LPXN or luciferase gene, respectively. 24 hours after transfection, cells were exposed to a single dose irradiation of 4 Gy and total RNA was isolated at 1, 2, 24, and 48 hours after irradiation, reverse transcribed and subjected to qRT-PCR. Non-irradiated cells $(0 \mathrm{~h})$ were used as a control.

The qRT-PCR analysis clearly shows a radiation induced increase in ITGB1 expression in control (siLuc) transfected cells. This irradiation-induced upregulation of ITGB1 was significantly reduced by knockdown of LPXN. Thus, LPXN knockdown prevented irradiation-induced expression of ITGB1. Furthermore, ITGB1 expression levels stayed at approximately $60 \%$ in siLPXN-transfected cells, even after irradiation. The illustrated values represent an average of three independent experiments, which were measured in duplicate and normalized to the ITGB1 expression in control cells (0h). Stars are indicating statistical significance as judged by students t-test $\left(\mathrm{p} \leq 0.05\left({ }^{*}\right) ; \mathrm{p} \leq 0.001\left({ }^{* *}\right)\right.$ : $\mathrm{p} \leq 0.0001\left({ }^{* * *}\right)$.

\subsection{The interaction of LPXN and the adhesion molecule p120CTN}

Previous experiments from our research group showed that the expression of the adhesion molecule p120 catenin (p120CTN) negatively correlates with the expression of LPXN. In addition, LPXN was reported to localize to the nucleus and mediate the transcriptional activity of the androgen receptor (AR) in PCa cells by direct binding to the AR. However, if LPXN is able to bind directly or indirectly to promoter sequences of the p120 catenin gene, CTNND1, remained unclear.

In order to investigate the nuclear mechanisms of LPXNs influence on p120CTN, the promoter region of the CTNND1 gene was fragmented and cloned in front of the luciferase gene. Promoter fragments were chosen according to the findings from Mortazavi et al. (2010), who described several activating as well as repressing elements in close proximity to the transcriptional start site of CTNND1. Interestingly, this group also reported a SP1 binding site in the promoter region of the CTNND1 gene. This work was already started during my master thesis (Dierks, 2011). A total of ten fragments of the CTNND1 promoter region were generated, starting from $2 \mathrm{~kb}$ upstream to $300 \mathrm{bp}$ downstream of the transcriptional start site (TS) (Fig. 3.23). 


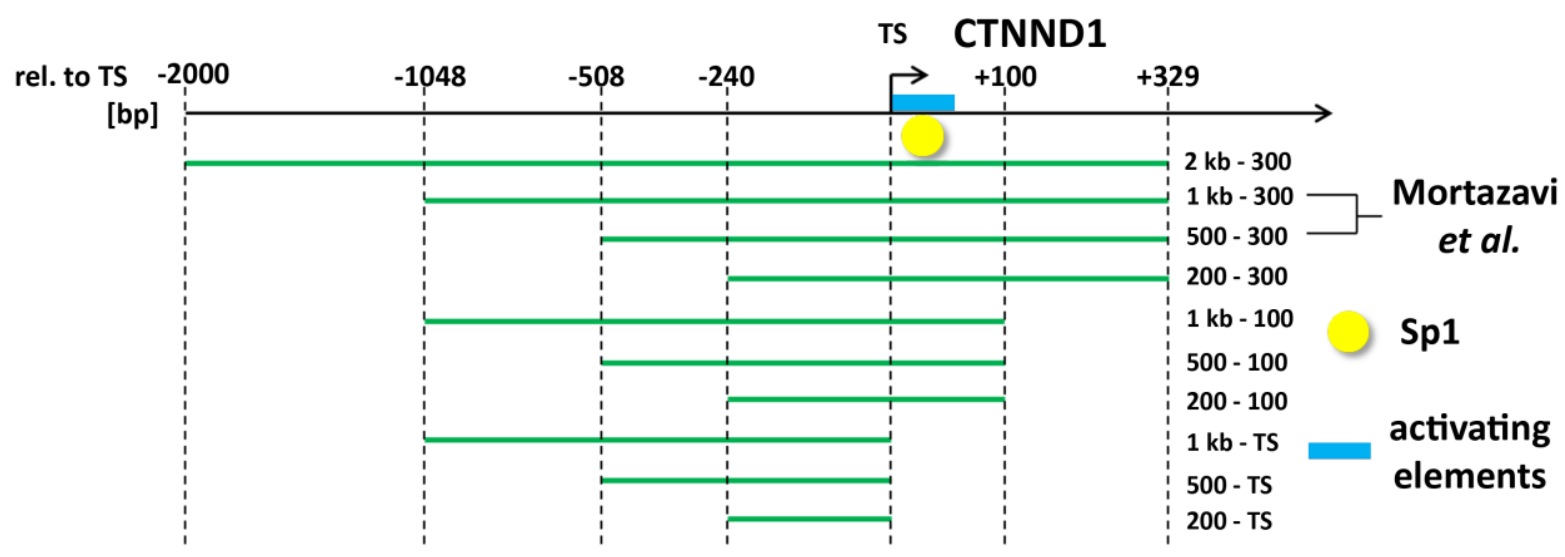

Fig. 3.23: Overview of the CTNND1 (p120CTN) promoter fragments. To analyze the CTNND1 promoter for LPXN responsive regions, a total of ten fragments were generated ranging from $2 \mathrm{~kb}$ upstream to $329 \mathrm{bp}$ downstream of the transcriptional start site (TS). The fragments were defined according to the findings from Mortazavi et al., who already reported activating elements and SP1 binding sites in the region around the TS of the p120CTN gene (CTNND1). The scheme displays the positions of the corresponding fragments.

Fig. 3.24 displays a restriction analysis using the XhoI and BglII restriction enzymes to check for successful integration of the corresponding promoter fragments into the luciferase reporter plasmid pGL4.16 luc2CP/Hygro.

p120ctn promoter fragments

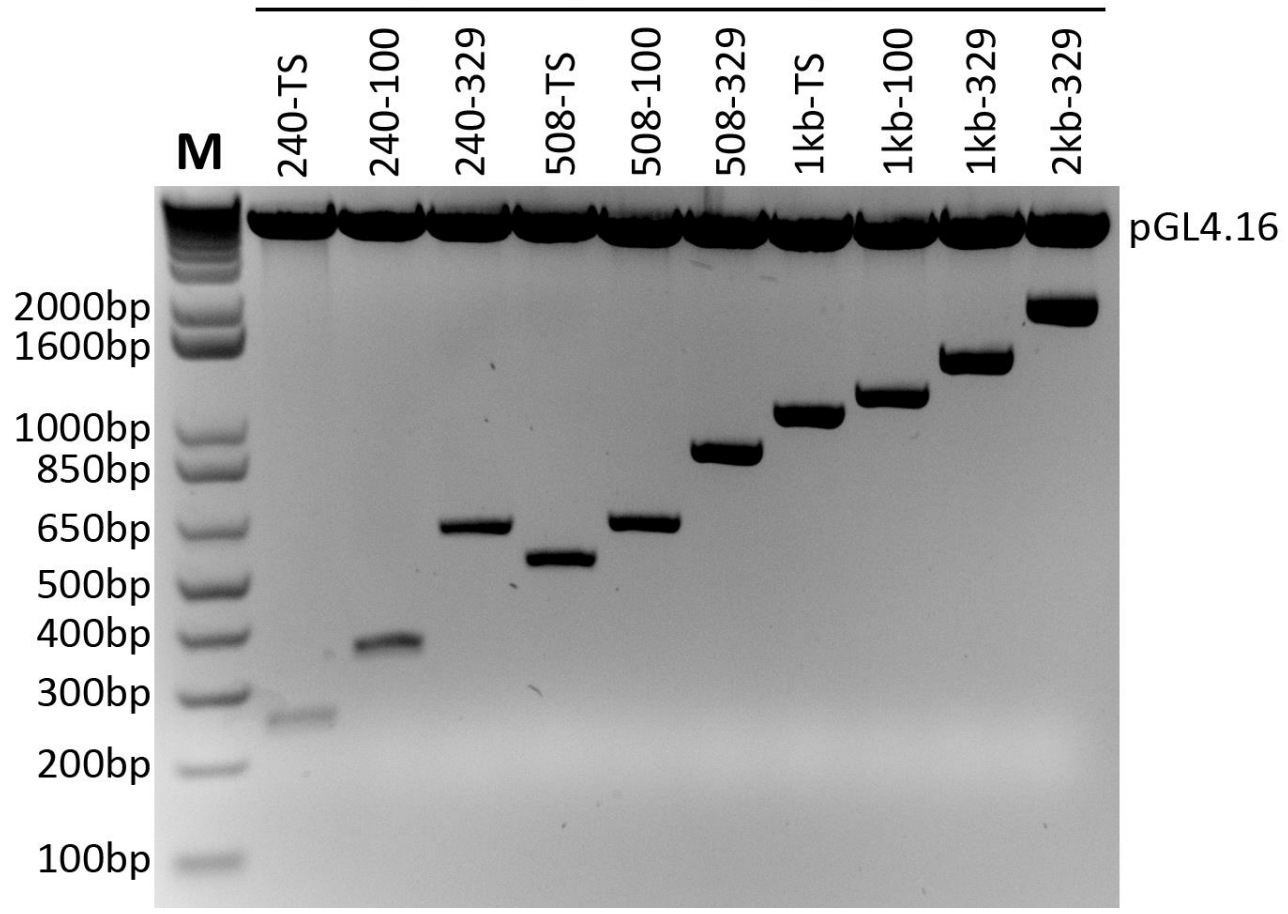


Fig. 3.24: Restriction analysis to show successful integration of the p120CTN promoter constructs into the pGL4.16 luc2CP/Hygro plasmid. The restriction enzymes XhoI and BgIII were used to cut the promoter fragments out of the luciferase reporter plasmid pGL4.16 luc2CP/Hygro to show successful integration. The agarose gel shows the estimated size of the fragments excised.

\subsubsection{The CTNND1 promoter is influenced by LPXN}

To analyze the activity of the CTNND1 promoter fragments, PC-3 cells were transfected with siRNA against the LPXN and luciferase gene, respectively. Since, LPXN and p120CTN expression are negatively correlating, this should reveal LPXN-responsive fragments of the CTNND1 promoter region in the subsequent luciferase reporter assays. The fragment ranging from $-200 \mathrm{bp}$ upstream to $100 \mathrm{bp}$ downstream of the transcriptional start site (TS) turned out to generate the highest difference in luciferase activity between siLPXN and control transfected cells. Interestingly, the fragments that were including downstream sequences to $100 \mathrm{bp}$ showed the highest promoter activity compared to fragments ranging to $300 \mathrm{bp}$ downstream, whereas constructs ending at the TS only showed activity comparable to background levels. In addition, promoter activity increased with increasing size of the fragments, but LPXN seems to have repressive influence on the more distant regions upstream of the TS (Fig. 3.25).

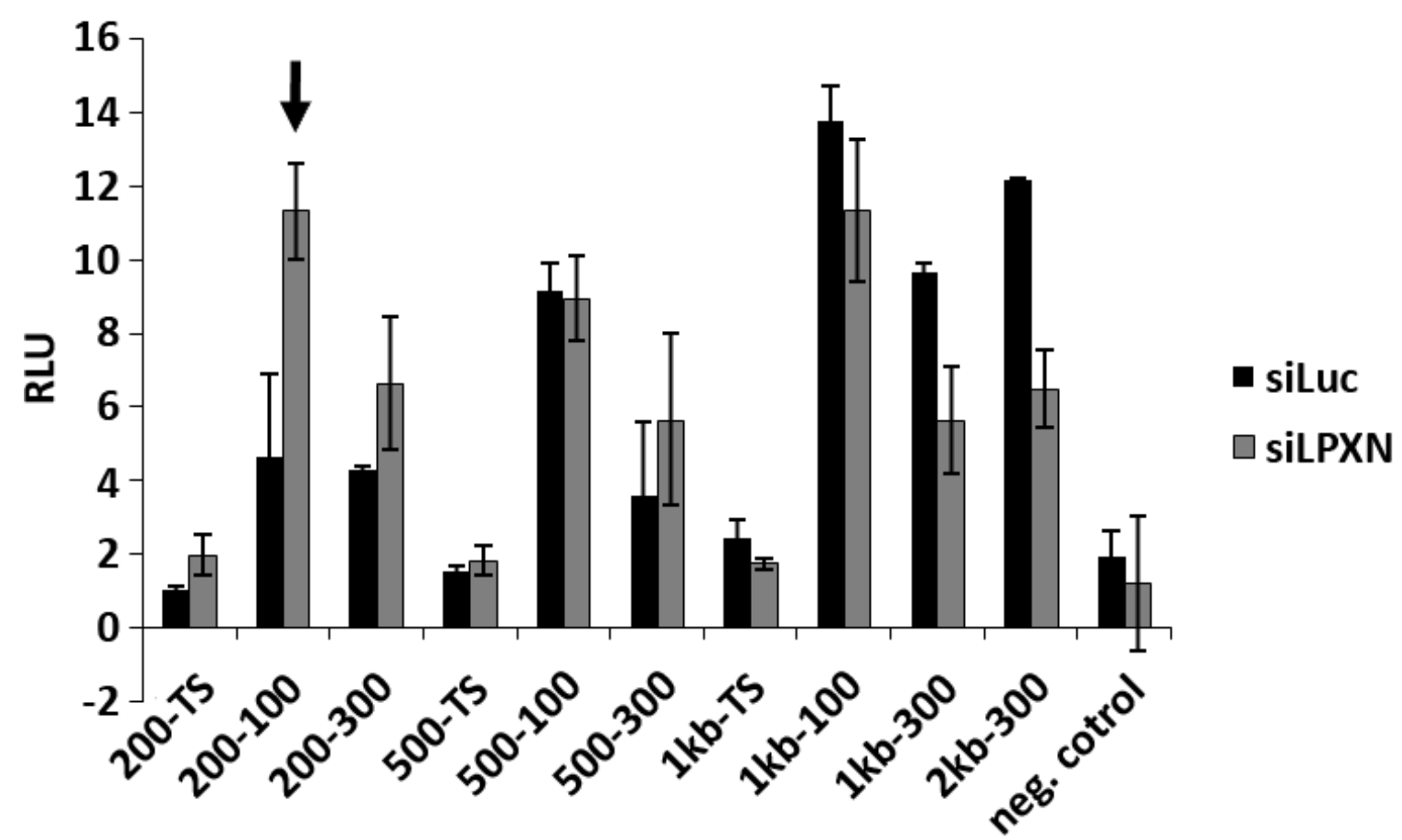

Fig. 3.25: Luciferase reporter gene assay of CTNND1 promoter fragments. For the identification of a LPXN responsive region in the CTNND1 promoter, the reporter plasmids containing the corresponding 
fragments were transfected into PC-3 cells that have been transfected with siRNA against the LPXN gene (siLPXN). SiRNA against the luciferase gene served as a control. Measurements of luciferase activity revealed that fragments including larger regions showed higher promoter activity, whereas the fragment ranging from $200 \mathrm{bp}$ upstream to $100 \mathrm{bp}$ downstream of the transcriptional start site (TS) showed the highest response to LPXN knockdown, which was not visible in control transfected cells. Therefore, this fragment was chosen for further analysis. Interestingly, fragments that did not include downstream sequences of the TS, showed only weak activity that was comparable to background levels. The illustrated values represent an average of three independent experiments. RLU = relative light units

\subsubsection{LPXN does not directly bind to promoter sequences of the CTNND1 gene}

In previous studies we could show that LPXN expression is negatively correlating with p120CTN expression (Kaulfuss et al. 2009). However, a regulatory mechanism was not purposed. ARA55, a protein family member of LPXN, was described to bind directly to DNA sequences (Nishiya et al. 1998). Zyxin, another focal adhesion-associated LIMdomain containing protein, was also reported to do the same (Nix, Beckerle 1997). This and the fact that LPXN is able to shuttle between the nucleus and the cytoplasm (Sundberg-Smith et al. 2008) were raising the hypothesis of a nuclear function of LPXN. To identify potential binding sites for LPXN in the promoter region of the CTNND1 gene the CTNND1 promoter construct that showed the highest reporter activity (-240 to +329 bp relative to transcriptional start site) was divided into fragments of $35 \mathrm{bp}$ in length (Fig. 3.26). Fragments were overlapping 5 bp on 5 ' and 3 ' ends. To exclude binding between two fragments another fragment was spanning the $5^{\prime}-3$ ' connections. 
$-240 \mathrm{bp}$

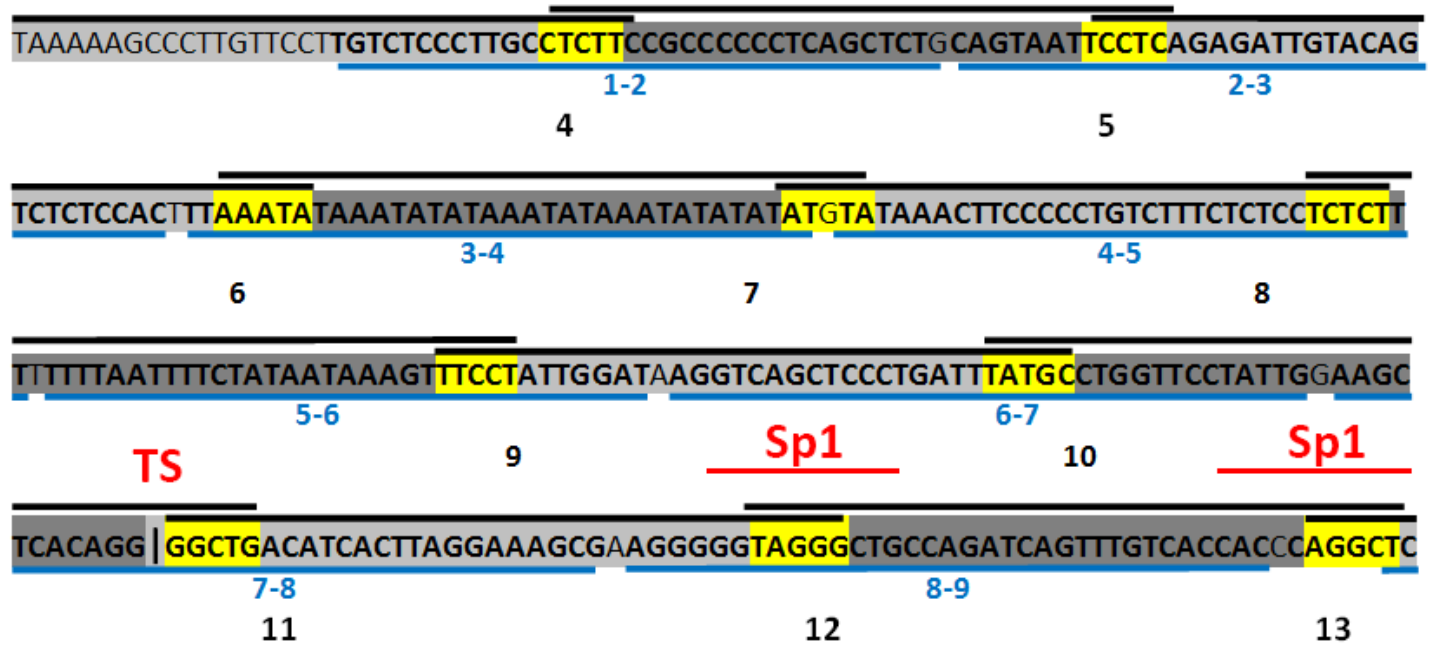

$\frac{\overline{\text { CCTTGCCTTIGGCTGGGTGCAACTTCCATTTAGGTGTTGGATCTGAGGGGGAAAAAAAAGAGAGAGGGAGAG }}}{9-10}$

14

15

\begin{tabular}{ccc}
\hline AGAGAGAAAGAGAGCAGGAAAGATCCCGAAGGAGGAAGAGTGGAAAAATCAACTGCCTGCTGGATT \\
\hline $11-12$ & $12-13$ \\
& 16 & 17
\end{tabular}

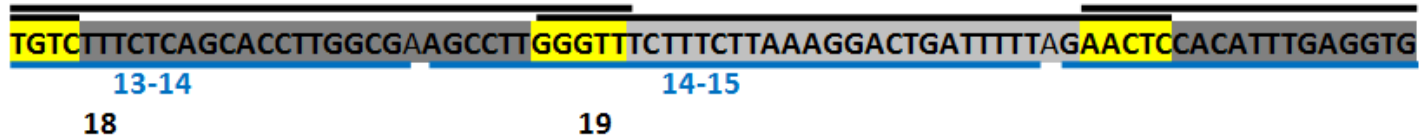

TGTGGCTTITGAAGAAAATGTATGTACTGACGGGAAAAGGAGGATA
15-16

Fig. 3.26: Scheme of the fragment of the CTNND1 promoter region for EMSA. The CTNND1 promoter construct reaching from 240 bp upstream to 329 downstream of the transcriptional start site was fragmented into $35 \mathrm{bp}$ fragments for an EMSA. The fragments were overlapping 5 bp on both 5' and 3' ends (black). In addition, fragments spanning the overlapping region (blue) were generated to exclude possible binding sites in this region. Fragments are numbered beginning with fragment one at $-240 \mathrm{bp}$ and ending with fragment 19. First overlapping fragment was numbered 1-2 ending with 16-17. TS = transcriptional start site; SP1 = Specificity protein 1 binding sites are indicated in red.

These fragments were subjected to an electrophoretic mobility shift assay (EMSA), starting with fragments around the transcriptional start site (Fig. 3.26). For this experiment PC-3 cells, which show the highest LPXN expression among our PCa cell lines, were used. To even enhance the presence of LPXN in the nucleus, an EGFP-LPXNNLS fusion protein was expressed in the PC-3 cells, whereas an EGFP protein served as a control. Functionality of the fusion protein was not changed as evidenced by androgen receptor transactivation assay (Dierks, 2011). Nuclear protein extracts were incubated 
with ${ }^{33} \mathrm{P}-$ labeled oligonucleotides and run on a native gel. As a positive control ${ }^{33} \mathrm{P}$ labeled oligonucleotides containing either one or two gamma activated sites (GAS, 2xGAS) were incubated with protein lysates from HeLa cells that were stimulated for 45 min with $5 \mathrm{ng} / \mathrm{ml}$ interferon gamma (IFN $\gamma$ ) to induce STAT1 activation. Supershifts were induced by the addition of anti-LPXN or anti-STAT1 antibody, respectively. The EMSA experiments were performed in close cooperation with Prof. Dr. mult. Thomas Meyer (Department of Psychosomatic Medicine and Psychotherapy; Molecular Psychocardiology, University Medical Center Göttingen). LPXN did not bind to any of the fragments 8 to 19 , which harbored sequences close to the transcriptional start site and further downstream sequences (Fig. 3.27B). However, binding of the STAT1-GFP control protein to the GAS (M67) was clearly visible. The 2xGAS oligonucleotides carried two binding sites for STAT1, consequently a larger band was visible which could be supershifted and confirmed the identity of the STAT1-GFP protein (Fig. 3.27A). In addition, an oligonucleotide with the $\mathrm{Sp} 1$ consensus sequence was designed and used in the EMSA. Incubation with the protein lysates from EGFP and EGFP-LPXN transfected PC-3 cells, did not show any differences in the band pattern of the EMSA. Thus, LPXN is not directly binding to this promoter region of the CTNND1 gene. 


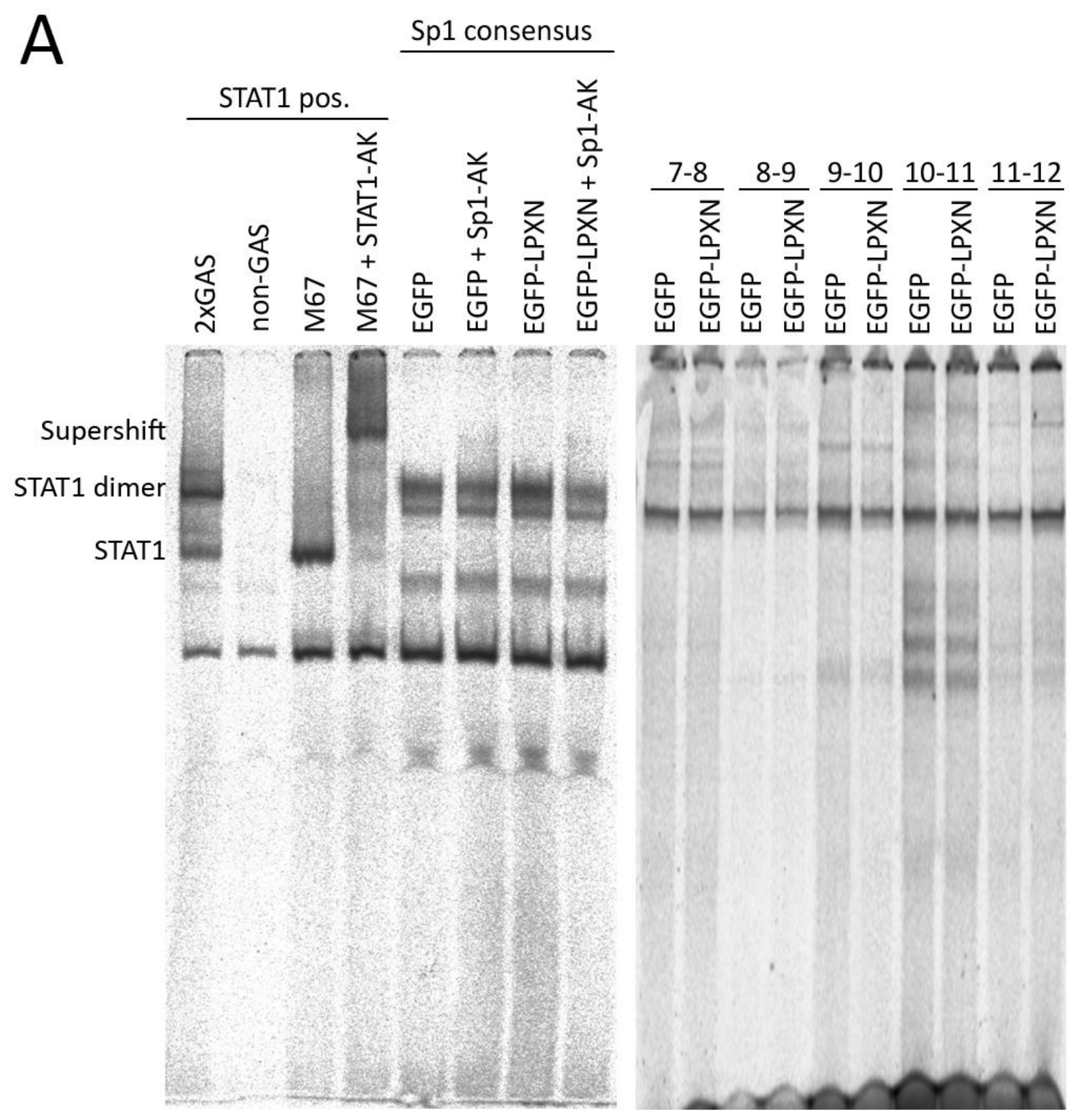




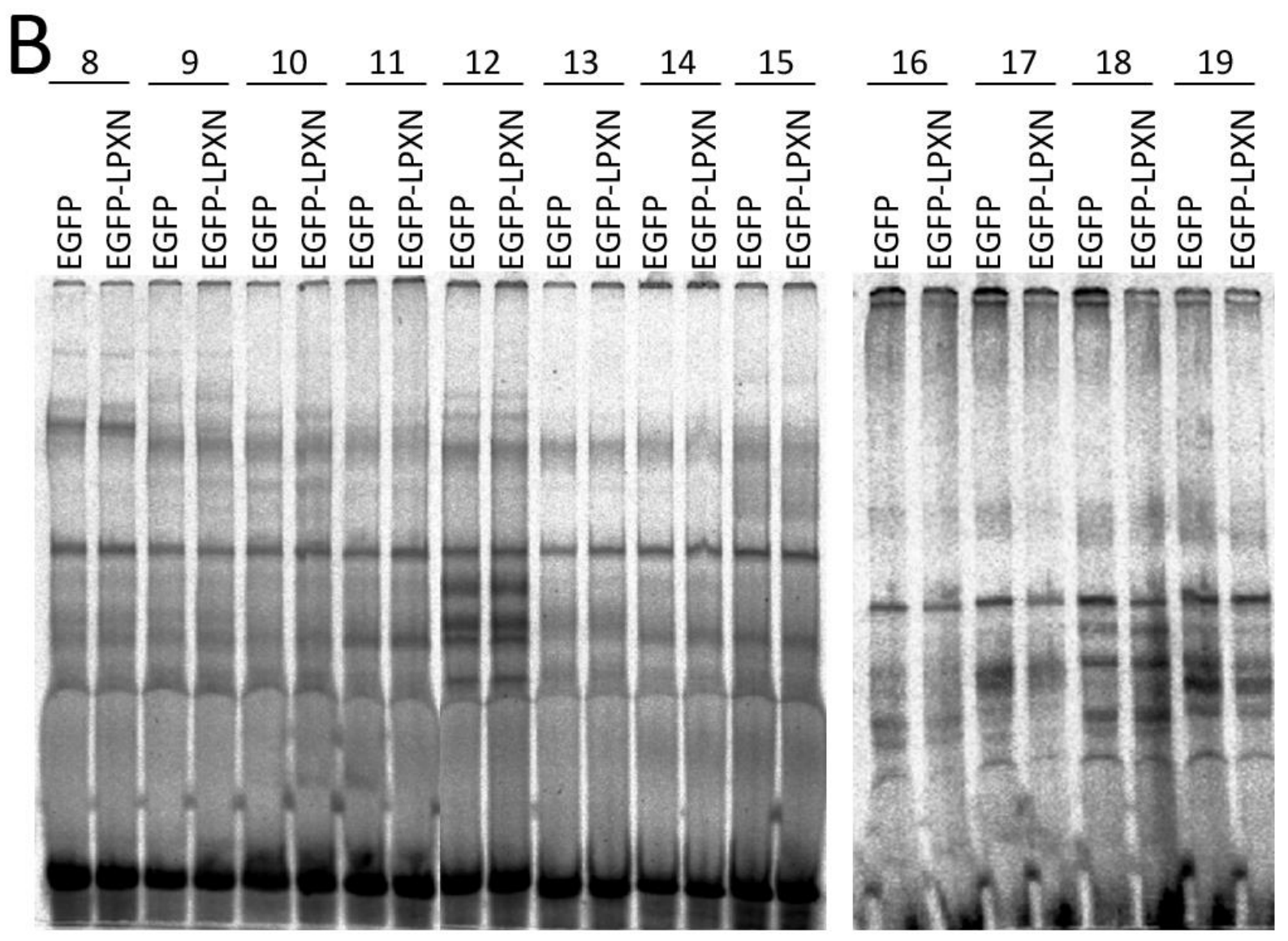

Fig. 3.27: CTNND1 promoter sequence-binding analysis of LPXN using EMSA. To investigate if LPXN is directly binding to the CTNND1 promoter region, whole protein lysate from PC-3 cells that were transfected with either EGFP or EGFP-LPXN-NLS constructs was incubated with ${ }^{33}$ P-labeled 35 bp oligonucleotides from the CTNND1 promoter. (A) As a positive controls a STAT1 binding site probes were incubated with protein lysates from HeLa cells that were stimulated with IFN $\gamma$. Probe M 67 contained one STAT1 binding site, probe 2xGAS contained two STAT1 binding sites. Supershift was induced by the addition of a STAT1-specific antibody. Likewise, a Sp1 consensus probe was incubated with lysates from PC-3 cells, but did not show any binding nor a supershift. Overlapping oligonucleotides from 7 to 12 were incubated with the above mentioned protein lysates, but did not show binding of EGFP-LPXN. (B) ${ }^{33}$ Plabled oligonucleotides 8 to 19 were also incubated with protein lysate from EGFP or EGFP-LPXN transfected PC-3 cells, respectively. As observed for the overlapping fragments these oligonucleotides did not reveal any binding sites for LPXN. 


\subsection{Generation of a LPXN gene trap conditional knockout mouse model}

\subsubsection{The LPXN targeted trap construct}

To investigate the function of LPXN in mammals a LPXN gene trap mouse line was generated in our laboratory. Since the induced phenotype by LPXN knockout cannot be predicted, a conditional knockout model was chosen. For the generation of this conditional knockout mouse model of LPXN, a mouse embryonic stem cell (mES) clone with a target trap construct was purchased from the European Conditional Mouse Mutagenesis Program (EuCOMM) (HEPD0688_2_D07). These cells are carrying the EuCOMM gene trap construct, which targeted the critical fourth exon (ENSMUSE00000445728), present in all transcript variants of the LPXN gene. The location of the loxP sites in this construct is leading to an excision of exon four after crerecombination and consequently to a loss of function of the LPXN gene. In addition, the cells carry a reporter cassette with a splice acceptor (SA) site that is flanked by FRT-sites and driven by the endogenous LPXN promoter, meaning that the endogenous expression of LPXN can be easily visualized by lacZ staining when the reporter cassette is not removed by Flp-recombinase (Fig. 3.28).

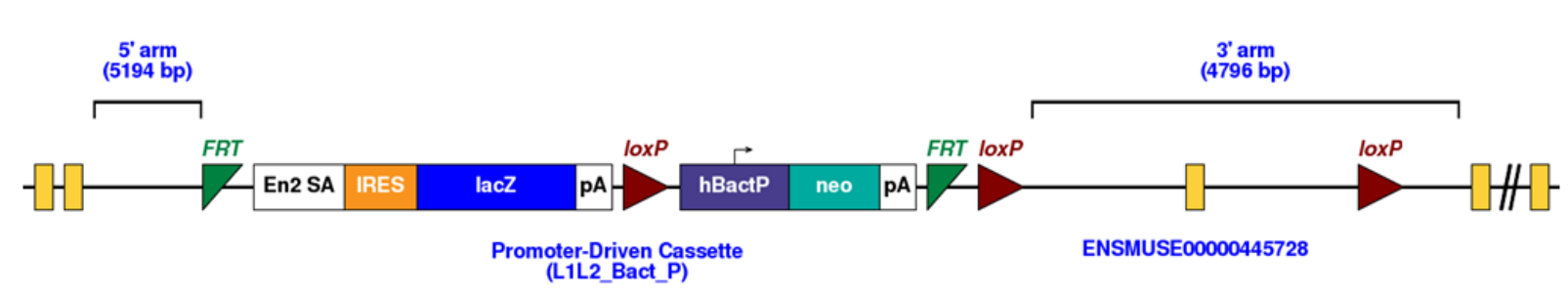

Fig. 3.28: Scheme of the LPXN gene trap construct. Showing the typical elements of the gene trap construct: A 5'-splice acceptor sequence, an internal ribosomal entry site (IRES), the reporter gene, lacZ, and a neomycin resistance gene, FRT-sites to remove the reporter/selector cassette to create the conditional allele, as well as loxP-sites flanking the neomycin-resistance gene and the critical exon. Picture was taken from EUCOMM. 


\subsubsection{Excision of reporter/selector cassette}

The typical gene trap construct consists of a splice acceptor (SA) sequence, a "trap" cassette, which includes the reporter gene (lacZ) as well as a selection marker $\left(\mathrm{Neo}^{\mathrm{R}}\right)$ and a polyadenylation (polyA) signal. Consequently, the translated $m R N A$ will generate a truncated protein consisting of the endogenous $\mathrm{N}$-terminal protein sequence lacking the exons downstream of the insertion site and the reporter protein.

For the generation of a conditional knockout the reporter/selector gene cassette has to be removed. In order to excise this cassette, mES cell clone HEPD0688_2_D07 was transfected with a pCAGGS-FLPe vector (Schaft et al. 2001) to express the FLPrecombinase, which excised the FRT-site flanked reporter/selector cassette. The transfection of the mES cells with the FLP-recombinase construct was done in cooperation with Prof. Dr. Ibrahim Adham (Department of Human Genetics, Göttingen). Genotyping of transfected mES cell clones was conducted using specific primers LPXN-5' and LPXN-3' that flanked the reporter cassette. PCR conditions were chosen in a way that the reporter cassette could not be amplified when no recombination took place. Only when the FLP-recombinase excised the reporter cassette a 304 bp product was amplified from the mutant sequence displayed in the scheme in Fig. 3.29 (mut). A 220 bp fragment was amplied from the wild type sequence (wt). Fig. 3.29 illustrates the band pattern of the pCAGGS-FLPe transfected clone 19 with a $304 \mathrm{bp}$ fragment from the mutant allele (reporter cassette excised) and a $220 \mathrm{bp}$ fragment amplified from the wild type allele $(\mathrm{wt})$.

Clone 46, which was not transfected with the pCAGGS-FLPe vector, only showed the product amplified from the wild type sequence at $220 \mathrm{bp}$ (wt) (Fig.3.29).

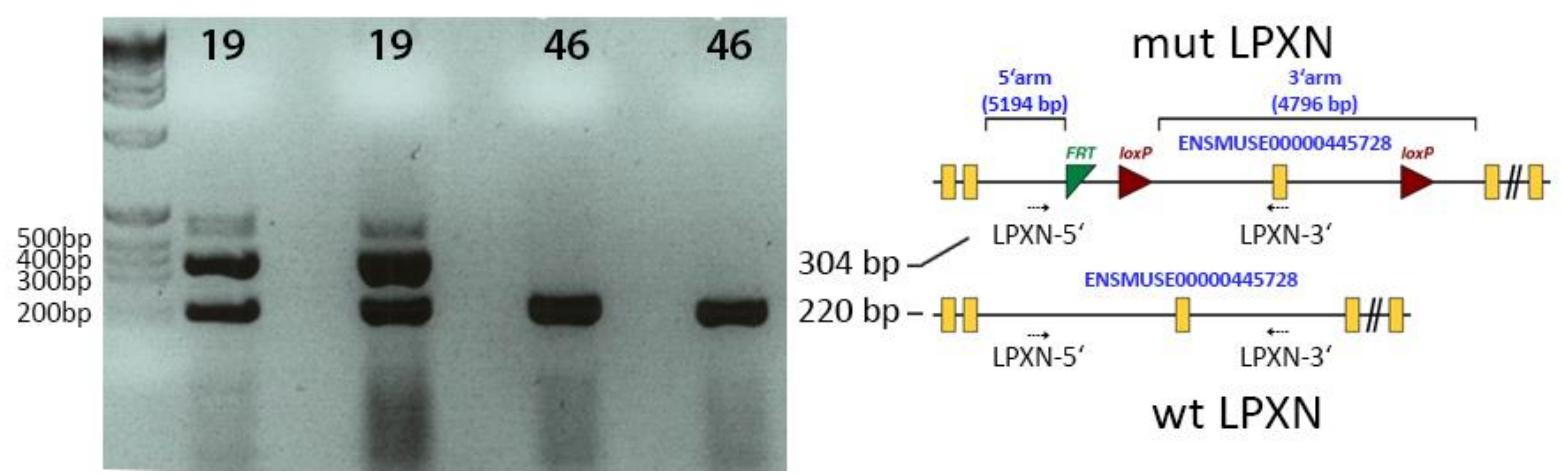


Fig. 3.29: Genotyping PCR of FLP-recombinase transfected and un-transfected mouse embryonic stem cells (mES). The numbers are indicating the clone number. Clone 19 was transfected with a pCAGGS-FLPe vector to express FLP-recombinase, whereas clone 46 served as an un-transfected control. Gene-specific primers LPXN-5' and LPXN-3' were used on gDNA isolated from the mES cell clones. Excision of the reporter/selector cassette is visible in the band pattern of clone 19, a wild type band at 220 bp and a mutant band at $304 \mathrm{bp}$, whereas clone 46 (un-transfected) only showed a wild type band at 220 bp. The scheme shows the corresponding alleles after FLP-recombination and the location of the primer pair. PCR conditions were chosen in a way that the reporter cassette could not be amplified from the mutant allele without recombination.

\subsubsection{First blastocyst injection of mouse embryonic stem cells}

In order to generate chimeric mice with the conditional LPXN allele, mES cells were injected into C57/BL6 wildtype blastocysts and transferred into C57/BL6 wildtype foster mothers. The purchased parental mouse embryonic stem cell line JM8A3.N1, derived from C57/BL6 with agouti fur color provided the advantage of easy identification of chimeric animals by coat color. The blastocyst injection and transfer were performed in cooperation with Dr. Ursula Fünfschilling (Max-Planck-Institute of Experimental Medicine, Göttingen).

Out of 5 blastocyst transfers we obtained only one chimera, which presented a low degree (0-10\%) of chimerism estimated from coat color. Mice born from this chimera, which was bred with C57/BL6 wild type mice, did not show the specific band pattern for the conditional allele as tested by genotyping. Therefore, no knockout mice were obtained indicating that there was no germline contribution in the chimeric mouse.

\subsubsection{Cytogenetic analysis of mouse embryonic stem cells for blastocyst injection}

Since trisomy, especially the trisomy of chromosome 8 , is one of the most common aneuploidies that counteracts successful generation of transgenic mice from mES cells (Sugawara et al. 2006), the clones were subjected to a cytogenetic analysis including chromosome counting. Both, Flp-recombinase treated (LPXN19) and non-treated (LPXN) mES cells were tested. Chromosome counting on metaphases of 20 cells of each cell population revealed 40 chromosomes $(2 \mathrm{n})$ in the majority of cells meaning that no significant numerical aberrations were present in both cell populations (Table 3.1). 
Table 3.1: Cytogenetic analysis of the mES cells after Flp-recombinase treatment.

\begin{tabular}{|r|l|c|c|c|c|c|}
\cline { 3 - 7 } \multicolumn{2}{c|}{} & \multicolumn{5}{|c|}{ Chromosome number (2n) } \\
\cline { 3 - 7 } \multicolumn{2}{|c|}{} & $\mathbf{4 3}$ & $\mathbf{4 2}$ & $\mathbf{4 1}$ & $\mathbf{4 0}$ & $\mathbf{3 9}$ \\
\hline Flp & LPXN (clone 19) & 0 & 0 & 1 & 16 & 3 \\
\hline non-Flp & LPXN (clone 46) & 2 & 0 & 0 & 17 & 1 \\
\hline
\end{tabular}

\subsubsection{Rescue of differentiated mES cells by RESGRO ${ }^{\circledR}$ culture medium}

Apart from aneuploidy, differentiation of ES cell lines is another cause for low percentage chimeras or poor germline transmission rates. Since cytogenetic analysis did not reveal any aneuploidies that could account for the low-percentage chimerism we observed from our first blastocyst injections, mES cells were cultured over two passages in the RESGRO ${ }^{\circledR}$ culture medium. With this special culture medium differentiated mES cells become distinguishable from undifferentiated mES cells. Culture and sub-cloning of undifferentiated mES cells were carried out in cooperation with Prof. Dr. Ibrahim Adham (Department of Human Genetics, Göttingen).

\subsubsection{Second blastocyst injection of mouse embryonic stem cells}

After mES cells were sub-cloned in RESGRO ${ }^{\circledR}$ culture medium, a second blastocyst injection was performed in cooperation with Dr. Ursula Fünfschilling (Max-PlanckInstitute of Experimental Medicine, Göttingen, Germany). This time, out of six blastocyst transfers a total number of three chimeras were obtained. One of them presented a high degree of chimerism, approximately $90 \%$, while the others were showing about $60 \%$ and $10 \%$ chimerism as estimated from coat color. All chimeric mice were male, reached sexual maturity and were mated with C57/BL6 wild type mice. The progeny was analyzed by genotype PCR for the presence of the 3'-loxP site. From the three chimeras only the one with the highest degree of chimerism (90\%) showed germline contribution

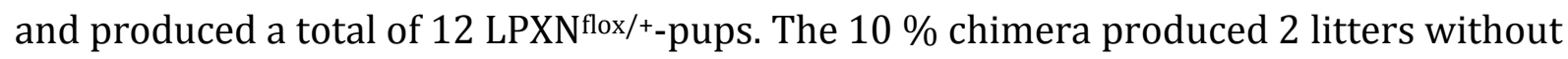


germline contribution, whereas the $60 \%$ chimera did not generate any offspring. Table 3.2 summarizes the offspring of the three chimeras.

Table 3.2: Table of chimera progeny

\begin{tabular}{|c|c|c|c|c|}
\hline Chimerism & Gender & Litter & positive & total pups \\
\hline $10 \%$ & $\sigma^{\lambda}$ & 2 & 0 & 16 \\
\hline $60 \%$ & $\sigma^{\lambda}$ & 0 & 0 & 0 \\
\hline $90 \%$ & $\sigma^{\lambda}$ & 4 & 12 & 25 \\
\hline
\end{tabular}

\subsubsection{Genotyping of LPXN19 chimera offspring}

To check for the presence of the 3'-loxP-site, a prerequisite for the conditionality of the allele (see Fig. 3.28) and for genotyping of the pups born from the chimeras, a pair of primers was designed. The forward primer LPXN-F was located in the critical exon 4 and the reverse primer (loxR) adjacent to the 3'-loxP-site. This will only produce a 343bp fragment from the mutant sequence, whereas no product is generated from the wild type sequence (Fig. 3.30). In addition, the presence of the 5'- and 3'-loxP-sites was confirmed by sequencing of the PCR products.

From all chimeric mice only the mouse with the highest degree of chimerism showed germline transmission. Genotyping of the pups of this litter showed one animal (27) that carried the specific band pattern for the conditional allele and the presence of the 3'loxP-site (Fig. 3.30). Mouse 27, as well as the positive tested candidates that were obtained from the following matings of this chimera, were used to establish a colony of the LPXN conditional knockout mouse line (LPXN19flox/flox). 


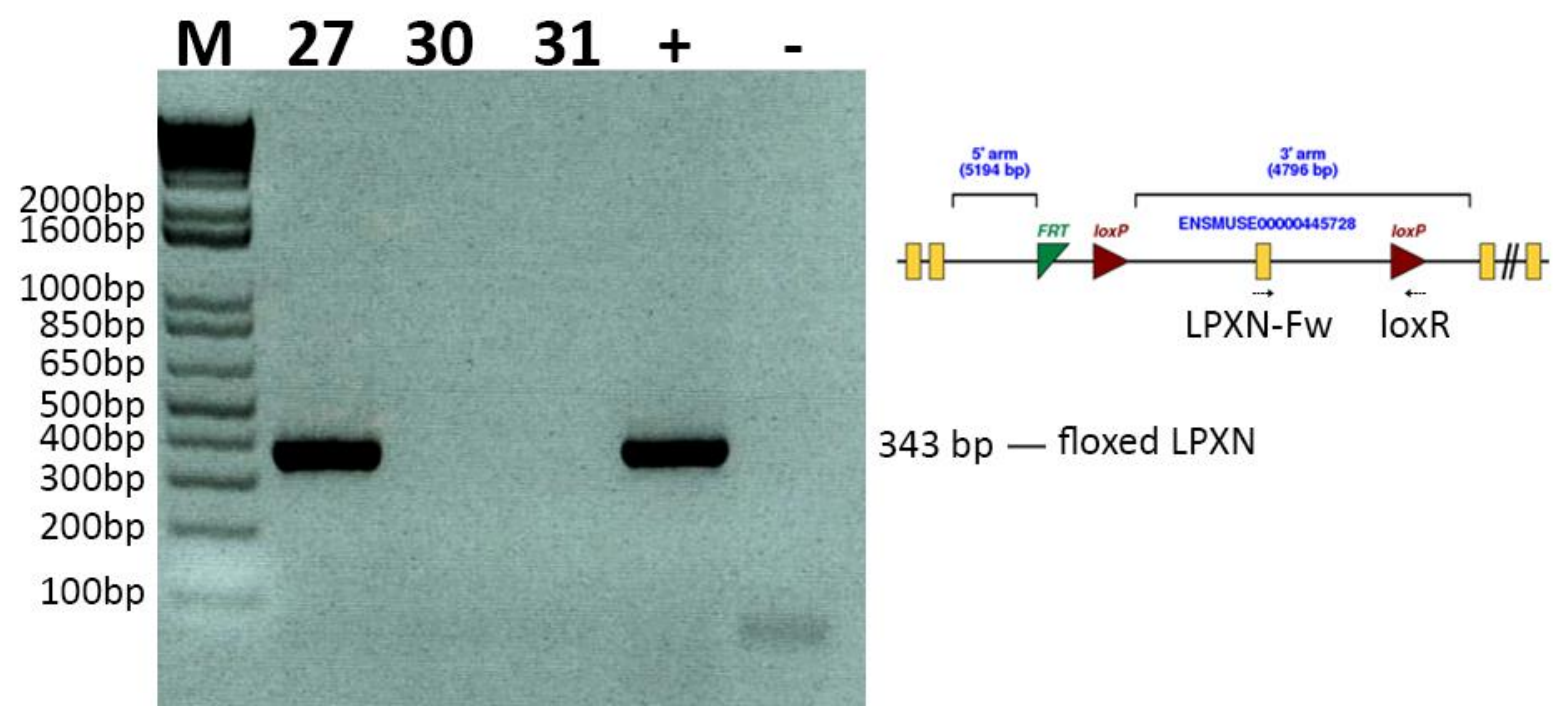

Fig. 3.30: Result of the genotyping PCR of LPXN19 F1 generation born from chimeric mice. The agarose gel shows the specific band patterns for both, the presence of the 3'-loxP-site using the LPXN-Fw and loxR primer pair. Only mouse 27 showed a specific band, whereas mouse 30 and 31 did not carry the mutant allele. The gDNA from mES cells served as positive control (+). For the negative control PCR $\mathrm{ddH}_{2} \mathrm{O}$ was used instead of DNA. $\mathrm{M}=$ Marker

\subsubsection{Establishment of a homozygous LPXN ${ }^{\text {flox/flox }}$ conditional knockout mouse model}

To establish a LPXN ${ }^{\text {flox/flox }}$ conditional knockout mouse line, heterozygous animals from the F1 generation of the chimera (LPXN19+/flox) were mated either inbred with their LPXN19+/flox siblings or with wild type C57/BL6 mice. For further breeding only the resulting homozygous $\mathrm{LPXN}^{\text {flox/flox }}$ animals were used to ensure a pure homozygous mouse line. Since the parental mES cells were also derived from the C57/BL6 mouse strain no backcrossing was required.

\subsubsection{Complete knockout of LPXN using the EIIA-Cre-loxP-system}

Since prediction of the phenotype of LPXN-/--animals is very difficult and it may also end in embryonic lethality, a conditional knockout model was chosen to keep the possibility of a tissue- and time-specific knockout of LPXN. To analyze LPXN function we aimed to disturb the LPXN gene very early in embryonic development. Therefore, LPXN19flox/flox mice were mated with EIIA-Cre ${ }^{+/+}$mice on a C57/BL6 background. The EIIA-Cre-mouse 
line expresses the bacteriophage P1 cre-recombinase under the control of an adenoviral promoter, which is already active before implantation of the oocyte into the uterus (Dooley et al. 1989). This early activity of the cre-recombinase excises the critical exon 4 of the LPXN gene very early in embryonic development. This gives rise to LPXN19+/flox/Cre+/- mice in the F1 generation that carry the heterozygous knockout only in those cells that expressed the cre-recombinase. Consequently, these mice are a mosaic for LPXN heterozygous knockout. Only when the recombination also reached the germline cells the knockout allele can be passed on to the F2 generation. To select for the $\mathrm{LPXN}^{+/-}$-mice, the mosaic mice were bred to C57/BL6 wild type mice. These LPXN19+/- mice of the F2 generation are used to establish the homozygous LPXN19 knockout line (Fig. 3.31).

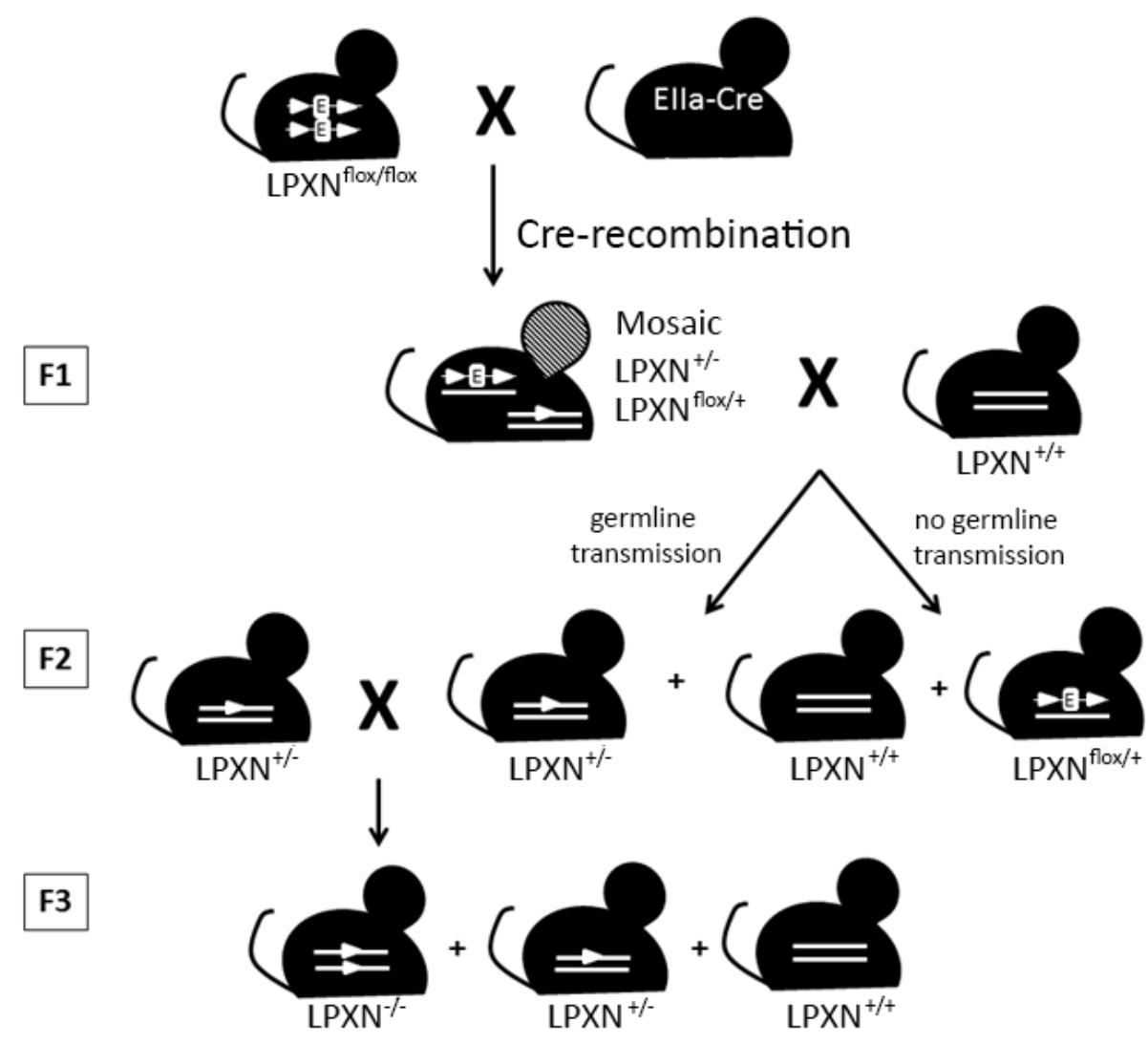

Fig. 3.31: Mating scheme to produce a LPXN $\%$ mouse line. To knockout LPXN very early in

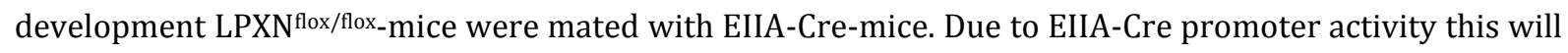
give rise to mosaic mice. These animals were bred with C57/BL6 mice to select for germline transmission of the knockout allele in the F2 generation. Finally, LPXN $\%$ animals were obtained in the F3 generation. 


\subsubsection{Genotyping of LPXN19 knockout mice}

To genotype putative LPXN conditional knockout mice, another pair of primers was designed. The specific primers mLPXN-In-3-4 \& mLPXN-In-4-5 amplified a 1114 bp product from the wild type sequence, approximately $1250 \mathrm{bp}$ from the LPXN19flox/flox allele and an approximately $500 \mathrm{bp}$ fragment from the knockout allele (Fig. 3.32). The $\mathrm{LPXN}^{\Delta /-}$ Crepos mice show three bands because of the EIIA promoter activity, which leads to mosaicism in these mice.

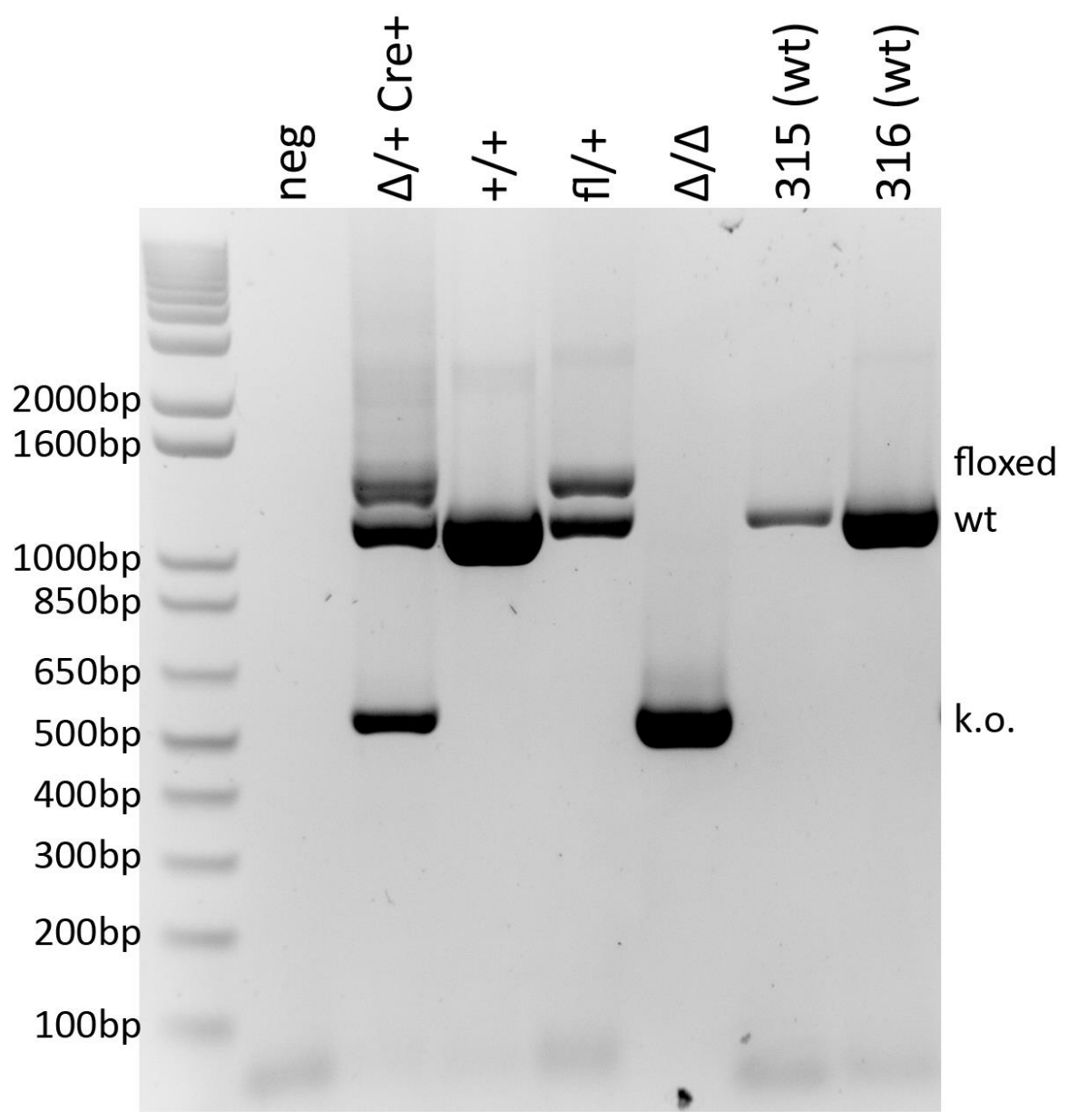

Fig. 3.32: Results of genotyping PCR of LPXN19 conditional knockout mice. Specific mLPXN-In-3-4 \& mLPXN-In-4-5 primers amplified an approx. 500 bp product from the knockout allele, an approx. $1250 \mathrm{bp}$ product from the floxed-allele and a $1114 \mathrm{bp}$ fragment from the wild type sequence. Due to the EIIApromoter activity, the $\Delta /+\mathrm{Cre}+$ mouse shows bands from all three alleles. For negative control $\mathrm{dd}_{2} \mathrm{O}$ was used. 


\subsubsection{Phenotypic analysis of LPXN19 knockout mice}

Macroscopic analysis of born $\mathrm{LPXN}^{-} /$mice did not exhibit any morphologic abnormalities (Fig. 3.33). LPXN//- mice were not distinguishable from C57BL/6 wild type mice.

\section{wild type}

\section{LPXN-/-}

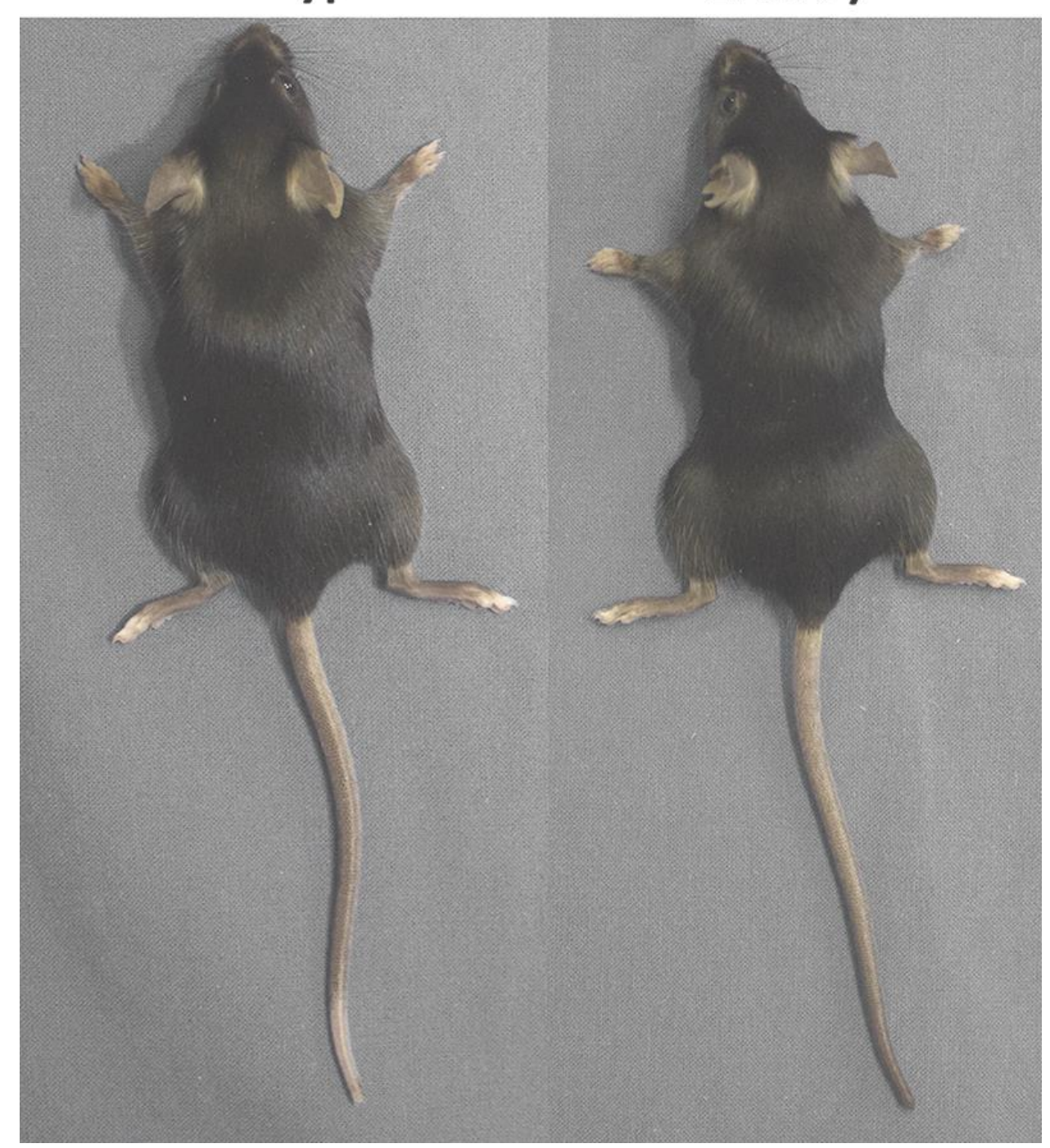

Fig. 3.33: Phenotypic comparison of a wild type C57BL/6 and a $\mathrm{LPXN}^{-} /$mouse. Comparison of 9 weeks old mice showed no obvious phenotype of LPXN $\%$ mice recognizable.

This result indicates that LPXN might not be essential during development. However, detailed studies have not been conducted so far and might reveal a certain phenotype later on. 


\section{Discussion}

\subsection{Summary of results}

In previous studies the actin-binding protein $\mathrm{CaD}$ was identified as a putative interaction partner of LPXN by a yeast-2-hybrid screening on a prostate cDNA library. In the present study this interaction was confirmed using co-immunoprecipitation and proximity ligation assays (PLA). Furthermore, this interaction was shown to be relevant for cancer cell migration. Using a combination of scratch and PLA assays, we detected increased interactions of LPXN and CaD in migrating PCa cells compared to non-migrating or confluent cells. In addition, the same experiments using an antibody that specifically detected the phosphorylated form of CaD showed similar results. Phosphorylated CaD detaches from actin leading to more dynamic actin structures. A specific localization pattern of LPXN and phospho-CaD interactions could be detected in migrating PCa cells. These interactions were located to the sub-membranous compartment, where the actin cytoskeleton needs to be more dynamic for cytoskeletal remodeling and were absent from the leading edge or stabilized actin structures of the migrating cell. Furthermore, we analyzed how LPXN influences the phosphorylation status of CaD. As it was shown that the activity of the extracellular signal-regulated kinase (ERK) was reduced after LPXN knockdown, we tested whether ERK is interacting with LPXN. A PLA was performed and revealed interaction of both proteins is present and enhanced in migrating cells. Moreover, the interaction pattern was resembling the pattern observed for pCaD and LPXN interactions. Interestingly, basal expression of ERK was equal among the PCa cell lines PC-3, DU145 and LNCaP, whereas activated ERK was only detectable in DU145 cells. Using the specific ERK inhibitor, PD98059, we identified the extracellular signal-regulated kinase (ERK) to be responsible for CaD phosphorylation. However, a simultaneous inhibition of ERK and siRNA-mediated downregulation of LPXN had no synergistic effect.

Previous studies showed that LPXN, as a focal adhesion protein, has great impact on cell adhesion. In the present study LPXN was shown to influence the expression level of a group of major adhesion molecules, the integrins. Especially, $\beta 1$ integrin expression was correlating with the expression of LPXN. In highly invasive PC-3 and DU145 cells $\beta 1$ 
integrin expression was high in comparison to the non-invasive LNCaP cell line. Downregulation of LPXN using siRNA reduced expression of $\beta 1$ integrin both on RNA and protein level, whereas overexpression of an EGFP-LPXN fusion protein in the PCa cell lines PC-3 and DU145, respectively, increased $\beta 1$ integrin expression. To analyze the basis for the LPXN-dependent expression of $\beta 1$ integrin, a Sp1 transcription factor (SP1) activity assay was performed and revealed that overexpression of an EGFP-LPXN fusion protein resulted in an increased activity of Sp1, whereas knockdown of LPXN expression did not show any significant changes in Sp1 activity.

LPXN expression of PCa cells was found to be inducible by ionizing irradiation. Expression levels started to increase at 2 hours and reached a peak at 48 hours after exposure to ionizing irradiation. Likewise $\beta 1$ integrin expression did also show a similar response to ionizing radiation. This effect was detectable both at RNA and protein levels. Downregulation of LPXN prevented an irradiation-induced upregulation of $\beta 1$ integrin and kept $\beta 1$ integrin expression levels at approximately $60 \%$ in comparison to unirradiated cells, which illustrates the influence of LPXN on $\beta 1$ expression. Furthermore, a downregulation of LPXN expression using siRNA resulted in a radiosensitization of the PCa cell lines PC-3, DU145 and LNCaP. In addition, blockade of $\beta 1$ integrin using the $\beta 1$ integrin inhibitory AIIB2 antibody resulted in the same radiosensitive response of the PCa cells. A simultaneous downregulation of LPXN and blockade of $\beta 1$ integrin did not reveal an additional or synergistic effect on the radiosensitization of the PCa cells.

In our research group the expression of the adhesion molecule p120CTN (CTNND1) was recently described to negatively correlate with LPXN expression. In the present study reporter gene assays using different fragments of the CTNND1 promoter showed that activating as well as repressing elements are present in the CTNND1 promoter and finally revealed a putative region for LPXN-mediated expression of p120CTN, ranging from $240 \mathrm{bp}$ upstream to $340 \mathrm{bp}$ downstream relative to the transcriptional start site of the CTNND1 promoter. For the subsequent EMSA, this region was further fragmented into 35 bp oligonucleotides. Nuclear extracts of PC-3 cells, which were additionally transfected with an EGFP-LPXN-NLS construct to enrich LPXN in the nucleus were used. The EMSA did not reveal any direct binding sites for LPXN in this CTNND1 promoter region.

The physiological role of LPXN in the organism is not clear and a knockout model for LPXN has not been reported. In this study a conditional LPXN knockout mouse model was generated. Therefore, mutant C57BL6 ES cells were purchased from EuCOMM that 
carried a targeted trap construct. After excision of the resistance/reporter cassette, these cells were injected into C57/BL6 blastocysts of agouti coat color to generate chimera. The first blastocyst injection only produced one chimera with low percentage chimerism and without germline transmission. Culture of the ES cells in RESGRO medium helped to remove differentiating ES cells from the culture. The second blastocyst injection generated three chimeras from which one displayed high percentage chimerism and germline transmission. The established LPXN conditional knockout mouse line was bred with the EIIA-Cre mouse model to mimic a loss of LPXN function very early in development. To date, LPXN/EIIA-Cre mice are viable, fertile and do not display any abnormalities during development.

\subsection{Paxillin proteins in cytoskeletal rearrangements and migration}

Tumorigenesis is a multi-step process starting with genetic changes that lead to the transformation of normal cells to tumor cells. These tumor cells will progress and end up in highly invasive and metastatic cells. The underlying molecular mechanisms that drive tumorigenesis and tumor progression are incompletely understood. However, these mechanisms might involve a variety of proteins such as $\mathrm{CaD}$ and small GTPases that facilitate actin-cytoskeletal remodeling or proteins of the integrin, paxillin and cadherin families that play a major role in the establishment and dynamics of focal contacts during cell adhesion.

Paxillin family proteins are predominantly localized to focal adhesion sites and share a characteristic domain structure, which grants them their roles as molecular adapter proteins (Schaller 2001). They overtake important cellular functions as they recruit a variety of proteins implicated in signal transduction or cytoskeletal rearrangement to focal adhesions. The regulation of these processes is essential for cell migration, which in turn is required for vital mechanisms like wound healing, immune response or embryogenesis. Actin-cytoskeletal dynamics during cell migration are tightly regulated by the Rho family of small GTPases (Jaffe, Hall 2005). Upon integrin ligation, paxillin becomes phosphorylated by SRC or FAK and regulates the activity of Rho GTPases by the recruitment of various guanosine exchange factors (GEFs), GTPase-activating proteins (GAPs) or effector proteins (Bellis et al. 1997; Burridge et al. 1992; Petit et al. 2000; Schaller, Parsons 1995). In addition, the CrkII-DOCK180-ELMO complex interacts with Y31 and Y118 phoshorylated paxillin to regulate signaling of the Rho family GTPase 
Rac1 during cell migration (Grimsley et al. 2004; Birge et al. 1993; Petit et al. 2000; Valles et al. 2004). On the other hand, phosphorylation of paxillin at the same residue was shown to regulate RhoA activity by binding to p120RasGAP (RASA1). This interaction reduces the affinity of p120RasGAP to p190RhoGAP (ARHGAP5) at the plasma membrane, which suppresses RhoA activity (Tsubouchi et al. 2002). Therefore, paxillin carries a major function during cytoskeletal rearrangements and largely affects the formation of protrusion at the leading edge of migrating cells (Fig. 4.1). Via its LD motifs paxillin binds to p95PKL (95-kDa paxillin-kinase linker), which mediates association of paxillin with another complex of the p21 GTPase-activated kinase (PAK), Nck and the guanosine exchange factor, PIX (Turner et al. 1999). Moreover, the same study showed interaction of this complex with the paxillin family protein ARA55. This protein was also described to co-localize with paxillin and $\alpha$-actinin at the ends of actin filaments of neonatal cardiac myocytes to regulate fetal gene expression and cytoskeletal organization (Yund et al. 2009). Another study showed that ARA55 reduced integrin-mediated cell spreading on fibronectin through competition with paxillin for FAK, which was reported as a novel mechanism of integrin-mediated signal transduction (Nishiya et al. 2001). Obviously, paxillin family proteins are involved in the regulation of cytoskeletal dynamics and provide a link between extracellular cues and intracellular response. Figure 4.1 summarizes the functions of the paxillin protein family members in cytoskeletal remodeling. Besides its indirect activation or inhibition of the Rho family GTPases Rac1 and RhoA, respectively, further associations and regulating functions of paxillin proteins have been reported, e.g. in cell spreading but these are not subject of the present study. 


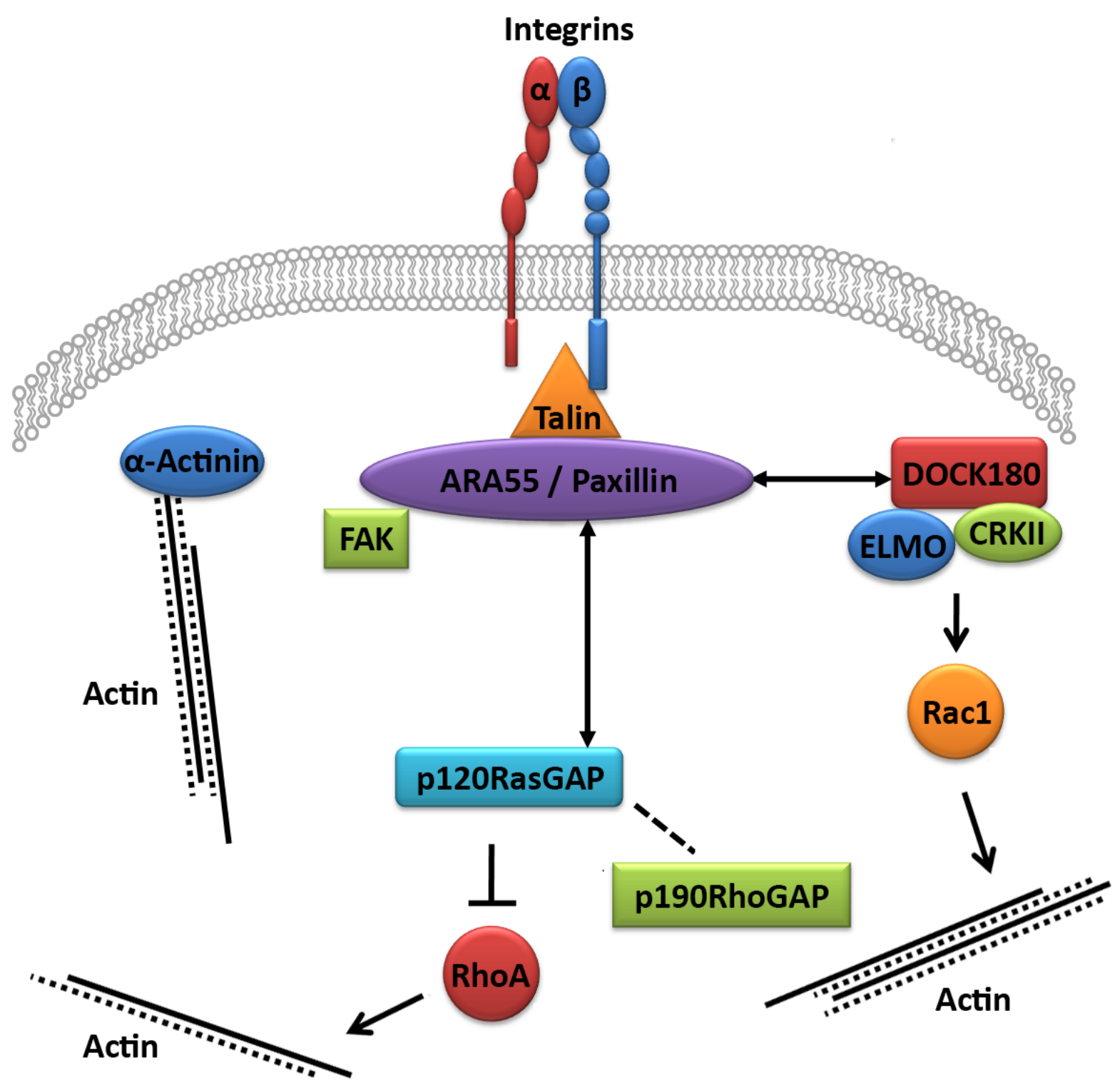

Fig. 4.1: Schematic overview of paxillin family members and their implications in cytoskeletal remodeling. The scheme shows a broad overview of how the paxillin proteins, ARA55 and paxillin are implicated in cytoskeletal rearrangements. Paxillin is acting on the family of small Rho GTPases via association with the GTPase activating protein p120RasGAP and the DOCK180/CRKII/ELMO protein complex. Small GTPases of the Rho family are important mediators of actin filament assembly and exhibit an essential role in cell migration. Furthermore, paxillin family proteins display a link between the tails of integrins and the actin cytoskeleton. Therefore, they include further structural proteins like talin or $\alpha$ actinin. For all paxillin proteins an interaction with FAK has been reported. As a protein predominantly localized at focal adhesions, FAK is of major importance for cell migration.

Paxillin's interaction with the focal adhesion kinase (FAK) and the structural adhesion protein vinculin are reported to be important for phosphorylation of paxillin and its subsequent localization to nascent focal adhesions. In addition, studies on the survival and motility of vinculin $\%$ cells showed that vinculin is modulating the interaction of 
paxillin and FAK and that this interaction in the absence of vinculin is up-regulating the activity of the extracellular signal-regulated kinase (ERK) (Subauste et al. 2004). The transforming oncoprotein bovine papilloma virus type 1 (BPV-1) E6 was demonstrated to interact with paxillin. Moreover, it was shown to disrupt actin stress fibers by blockade of paxillin interaction with vinculin and FAK at its LD motifs ((Tong et al. 1997). The human papilloma virus type 16 (HPV-16) E6 protein also interacts with paxillin at the same site of the LD motifs and additionally targets degradation of the tumor suppressor protein p53 together with the ubiquitin-protein ligase E6-AP (Vande Pol, S B et al. 1998). In addition, paxillin was demonstrated to interact with actopaxin, a protein that is directly binding to F-actin. This interaction was suggested to be important for integrin-dependent remodeling of the actin cytoskeleton during cell motility and cell adhesion. However, the exact nature of this interaction is still elusive (Nikolopoulos, Turner 2000). Interestingly, actopaxin was reported to influence cell invasiveness and motility by regulating the expression of focal adhesion proteins, including paxillin, in hepatocellular carcinomas ( $\mathrm{Ng}$ et al. 2013). Thus, paxillin binds to a variety of proteins involved in changes in the organization of the actin cytoskeleton. For LPXN it is not much known about its associations with cytoskeletal proteins. The only reported association was found in murine osteoclasts, where LPXN binds to the paxillin-kinase linker, p95PKL, an ARF-GTPase-activating protein (Gupta et al. 2003).

LPXN was found to interact with other focal adhesion proteins like the focal adhesion kinase pp125FAK, the protein tyrosine kinase PYK2 or the protein phosphatase PTPPEST that take part in signal transduction (Gupta et al. 2003; Sahu et al. 2007b). In contrast, LPXN was shown to bind and co-activate the androgen receptor as well as the sarcoma tyrosine kinase, SRC (Kaulfuss et al. 2008; Sahu et al. 2007a). However, these interactions had no effect on cytoskeletal rearrangements. Since proteins of the paxillin protein family share great homology especially in their protein domains, it seems likely that LPXN is able to interact with several other interactions partners that have not been identified so far and might act as potential facilitators of cytoskeletal remodeling. 


\subsection{Interaction of LPXN and CaD and its implications in prostate cancer}

\subsubsection{Caldesmon and its role in cytoskeletal rearrangements}

Caldesmon (CaD) is a myosin-, actin-, tropomyosin-, and $\mathrm{Ca}^{2+}$-calmodulin-binding protein involved in the regulation of contractility and actin cytoskeleton remodeling in smooth muscle and non-muscle cells. The CALD1 gene is alternatively spliced to generate two major isoforms of the CaD protein (Haruna et al. 1993). A high molecular mass $\mathrm{CaD}(\mathrm{h}-\mathrm{CaD})$, which is almost exclusively expressed in smooth muscle cells and a low molecular mass CaD (l-CaD), which is ubiquitously expressed in nearly all cell types (Hayashi et al. 1992). Most evidence today is based on findings from h-CaD and only little is known about l-CaD. Since both isoforms are generated from the same gene, domain sequences are identical and their biochemical characteristics are expected to be quite similar. CaD stabilizes the actin cytoskeleton by binding to tropomyosincontaining actin filaments, which leads to effective inhibition of the actomyosin ATPase activity, the driving force in filament-contraction and -motility. Furthermore, CaD is known to regulate multiple actin-associated proteins. The actin-binding activity of the actin-bundling protein fascin is mediated by the association of $\mathrm{CaD}$ with tropomyosin in a $\mathrm{Ca}^{2+}$-calmodulin and phosphorylation-dependent manner (Ishikawa et al. 1998). Likewise, the affinity of filamin to F-Actin, a protein important for orthogonal crosslinking of actin filaments at the cortical cytoplasm, is regulated in a similar fashion (Nomura et al. 1987). In addition, CaD was shown to act on actin polymerization. During the nucleation process $\mathrm{CaD}$ is preventing F-actin-binding of the Arp2/3 complex, whereas $\mathrm{Ca}^{2+-}$-calmodulin-binding and/or phosphorylation of $\mathrm{CaD}$ diminished this inhibitory effect (Yamakita et al. 2003). This actin polymerization process plays an important role in the regulation of motile structures like membrane ruffles and lamellipodia (Lin et al. 2009). Thus, CaD might display two opposing functions. On the one hand $\mathrm{CaD}$ stabilizes the filamentous actin structures by direct binding along with tropomyosin, on the other hand $\mathrm{CaD}$ prevents the assembly of new actin filaments by competition for F-actin structures with the Arp2/3 complex and other actin-binding proteins like filamin and fascin. 
Controlling actomyosin ATPase activity and actin cytoskeleton remodeling is important for normal cell function. Therefore, the regulation of CaD activity is of fundamental interest for the cell. However, the essential functions of CaD are tightly associated with its affinity for actin. This affinity for actin is affected by the presence of $\mathrm{Ca}^{2+}$-calmodulin and by phosphorylation of a number of residues by various kinases. In smooth muscle cells contraction requires $\mathrm{Ca}^{2+}$. High levels of $\mathrm{Ca}^{2+}$ will lead to the activation of $\mathrm{Ca}^{2+-}$ calmodulin, which directly binds to and releases $\mathrm{CaD}$ from actin. In non-muscle cells actin binding of $\mathrm{CaD}$ is predominantly regulated by phosphorylation. Both isoforms $\mathrm{h}$ $\mathrm{CaD}$ and l-CaD are reported to be phosphorylated during a variety of cellular processes like smooth muscle contraction, migration, non-muscle cell division or cell transformation by oncogenic v-erbB (Adam et al. 1992; Kordowska et al. 2006; Cuomo et al. 2005; Matsumura, Yamashiro 1993). The phosphorylation of CaD results in reduction of its binding properties to its binding partners depending on the phosphorylation sites (Fig. 4.2A). Thus, upon phosphorylation the inhibition of the actomyosin ATPase activity is released. There are various phosphorylation sites found in the $\mathrm{CaD}$ protein and $\mathrm{a}$ number of kinases have been identified to specifically phosphorylate these residues. Hence, phosphorylation sites important for actin binding are located in the actin-binding domain (Foster et al. 2000). Kinases that are known to phosphorylate Serine 789 of h$\mathrm{CaD}$ or Serine 534 of $\mathrm{l}-\mathrm{CaD}$ are the cdc2 kinase, the p38 mitogen-activated protein kinase (MAPK) and the extracellular signal-regulated kinase (ERK), whereas, cdc2 is associated with CaD phosphorylation during mitosis (Li et al. 2003; Mak et al. 1991). Another study showed that activation of p38 MAPK and phosphorylation of l-CaD is fundamental for migration of tracheal smooth muscle cells in response to urokinase type plasminogen activator (Goncharova et al. 2002). In pulmonary artery smooth muscle cells, which express high levels of l-CaD rather than h-CaD, the activation of ERK and subsequent phosphorylation of $\mathrm{l}-\mathrm{CaD}$ is required for platelet derived growth factor (PDGF)stimulated cell migration (Yamboliev, Gerthoffer 2001).

l-CaD associates with actin stress fibers in non-muscle cells and is an essential component of podosomes, highly dynamic and actin-based structures in highly motile cells (Eves et al. 2006; Gu et al. 2007). Loss of l-CaD results in reduced stress fibers and focal adhesions and is found in many transformed and cancer cells including gastric cancer (Fig. 4.2B) (Novy et al. 1991; Owada et al. 1984; Ross et al. 2000; Tanaka et al. 1993). In diagnostics, h-CaD is used as a specific tumor marker to distinguish smooth muscle tumors from bone tumors with myeloid differentiation (Watanabe et al. 2000). 
However, the pathological role of $\mathrm{CaD}$ is not exactly clear. There are a number of conflicting results suggesting l-CaD to have a tumor-supporting as well as -suppressive role. Primary colon cancer and liver metastasis tissue showed increased expression levels of $\mathrm{l}-\mathrm{CaD}$, which was correlating with a poor response to chemoradiotherapy of these patients. Interestingly, suppression of l-CaD in human colon cancer cell lines increased the susceptibility to 5-fluorouracil (5-FU) (Kim et al. 2012). In contrast, podosome formation, a cellular structure that is formed during cell invasion, in transformed and cancer cells is affected by CaD expression. Ectopic expression of CaD showed a decreased ECM degradation and subsequently suppressed invasion. Furthermore, depletion of $\mathrm{CaD}$ facilitated podosome formation and enhanced cell invasion (Yoshio et al. 2007).
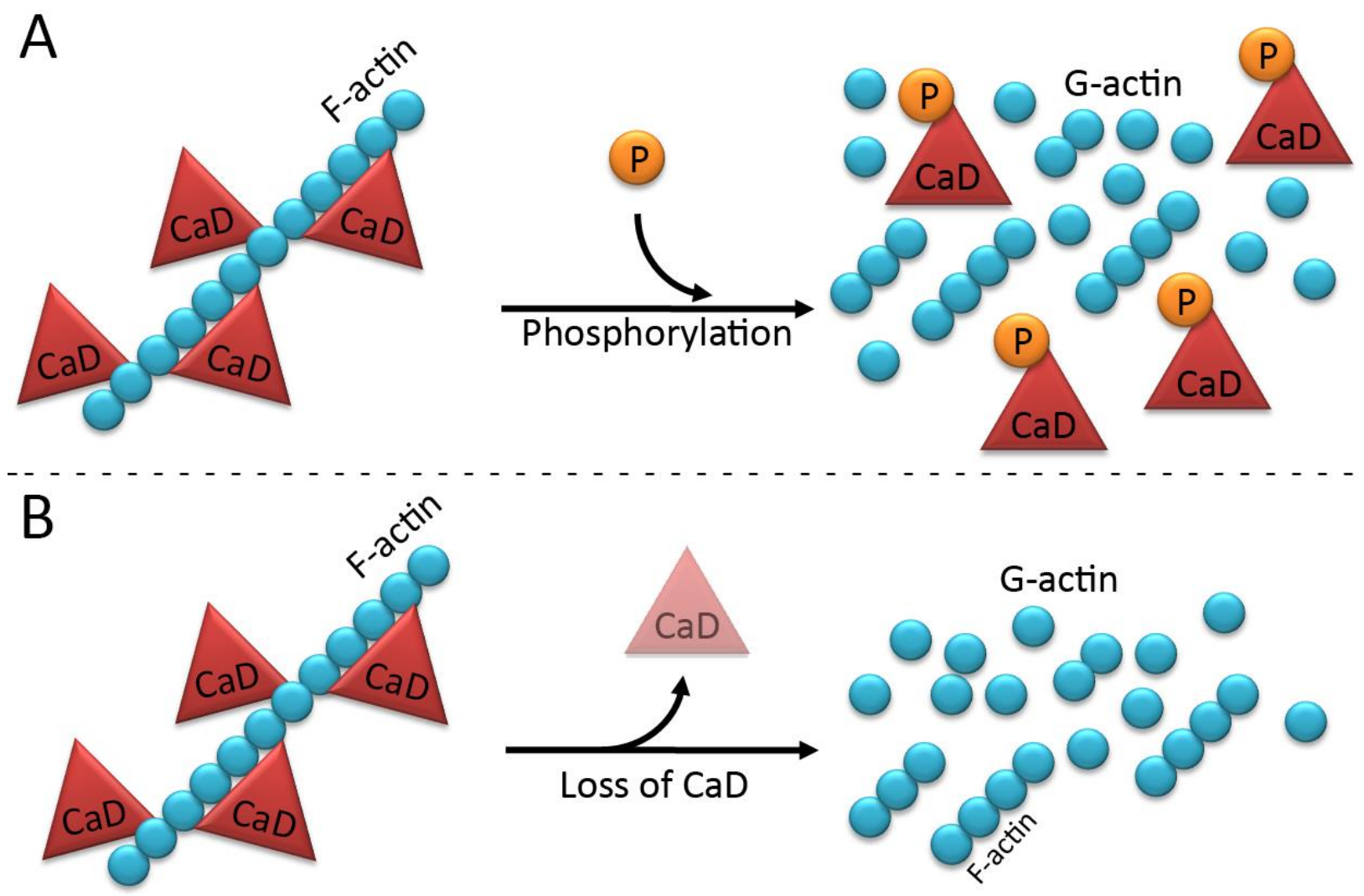

Fig. 4.2: Simplified schematic overview of $\mathrm{CaD}$ in actin stabilization in non-muscle cells. CaD binds to F-actin and stabilizes stress fibers. The activity of $\mathrm{CaD}$ and affinity for actin is mostly regulated through phosphorylation (A). Upon phosphorylation CaD reduces its affinity and detaches from actin stress fibers. As a result actin stress fibers are disassembled. (B) CaD binds and stabilizes actin and loss of CaD leads to the destabilization of actin stress fibers (Lin et al. 2009).

As revealed by siRNA-mediated knockdown of $\mathrm{CaD}$ in smooth muscle cells, CaD might not only play a role in the rearrangement of the actin cytoskeleton but also showed to 
have a regulatory function on other cytoskeletal structures. Knockdown of CaD decreased the amount of F-actin, whereas the expression of actin was not changed (Dierks et al. 2015). Interestingly, CaD might interact with intermediate filament components such as vimentin and desmin as evidenced by in vitro binding assays. Electronmicroscopic analyses of these cells showed that the distribution of the microfilaments was disturbed after CaD knockdown. The same study further showed that there were no alterations in expression or distribution of microtubules detectable in these cells (Deng et al. 2007). Furthermore, Ishikawa et al. found that non-muscle CaD from bovine brain is associating with microtubules and that this binding, similar to actin binding, is inhibited by the presence of $\mathrm{Ca}^{2+}$-calmodulin and/or the phosphorylation of CaD (Ishikawa et al. 1992a; Ishikawa et al. 1992b). Thus, CaD, as a mediator of cytoskeletal rearrangements, displays an important protein in many cellular processes such as cell shape, cell division, invasion and migration.

\subsubsection{LPXN modulates cytoskeletal rearrangements through interaction with CaD}

In our research group, LPXN was identified as a protein, which regulates the invasion of PCa cells and as an interaction partner of CaD. In the present study, this interaction was confirmed by co-immunoprecipitation and PLA. LPXN has a number of interaction partners, but has not been shown to directly interact with any cytoskeleton-associated protein. Therefore, CaD displays a putative candidate, which controls actin-cytoskeleton stabilization during invasion and migration of PCa cells downstream of LPXN.

Investigations from our research group showed that the phosphorylation status of $\mathrm{CaD}$ is extenuated by LPXN knockdown. In addition, we observed that the activity of TGF- $\beta$ activated kinase 1 (TAK-1) is also reduced after LPXN knockdown. However, this had no effect on its downstream targets JNK and p38 MAPK (von Hardenberg, 2010). Thus, ERK became the candidate kinase for LPXN-mediated phosphorylation of CaD during migration of PCa cells. Using a MEK1-specific inhibitor that blocks activation of ERK reduced phosphorylation of $\mathrm{CaD}$ and identified ERK to be responsible for $\mathrm{CaD}$ phosphorylation (Dierks et al. 2015). These results supported our hypothesis, which emphasizes LPXN as a mediator of CaD phosphorylation during PCa cell migration. In this hypothesis LPXN is recruiting ERK to phosphorylate l-CaD at S534, a 
phosphorylation site important for actin affinity. Once phosphorylated CaD detaches from actin, which leads to highly dynamic actin structures (Fig. 4.3). The major phosphorylation sites in h- and l-CaD are ERK-specific sites and are phosphorylated in response to migrative stimuli. The modulatory role of ERK-dependent phosphorylation of $\mathrm{CaD}$ in cell motility and migration has already been reported (Ishikawa et al. 1998). By this mechanism we could explain the pathological overexpression of LPXN, which is resulting in the aggressive growth and enhanced motility of metastatic prostate tumor cells (Dierks et al. 2015).

normal cell

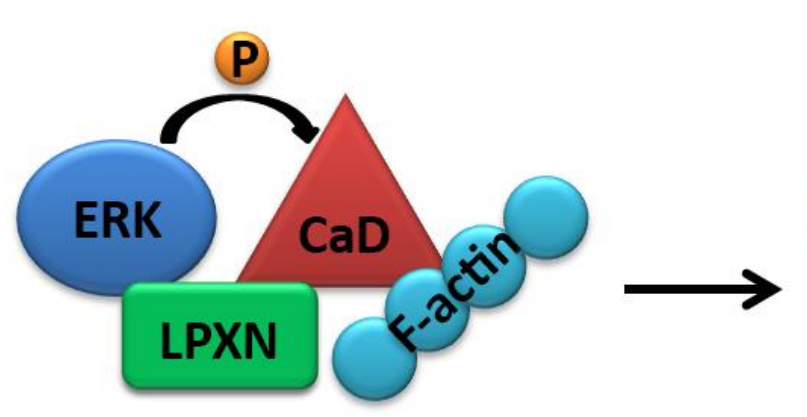

\section{migrating cell}

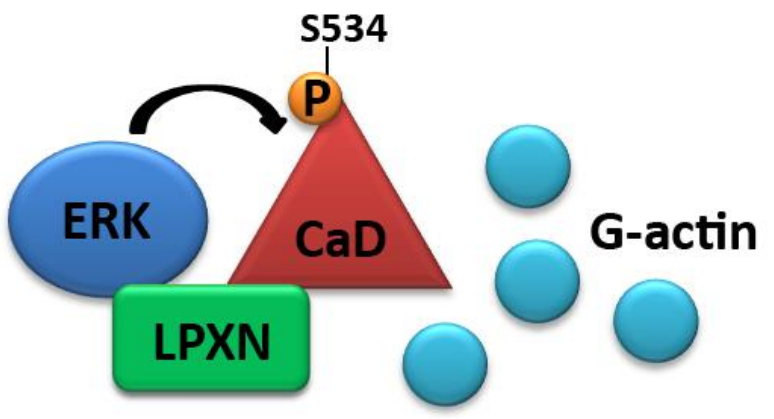

Fig. 4.3: Scheme of LPXN-mediated phosphorylation of CaD through the ERK kinase. In our hypothesis LPXN acts as a mediator of CaD phosphorylation during PCa cell migration. LPXN is recruiting the ERK kinase to phosphorylate $\mathrm{CaD}$ at places of pre-dynamic actin-cytoskeleton. Upon phosphorylation $\mathrm{CaD}$ detaches from actin leading to highly dynamic actin structures, which enables actin-cytoskeletal remodeling and consequently migration. This hypothesis could explain the pathological overexpression of LPXN in advanced PCa cells that may be responsible for their aggressive growth (Modified according to Matsumura et al. 1993, Wang 2008 and Dierks et al. 2015).

Using in situ PLA technique we could visualize the subcellular localization of the interactions of LPXN with CaD or ERK, respectively. In migrating cells the interaction pattern of LPXN and ERK was similar to that of LPXN and CaD. This pattern showed interactions mainly in the sub-cellular compartment, whereas they were absent from the membrane or leading edges of the cell. Thus the localization of the interactions can be divided into two major zones. On the one hand, a "pCaD-zone", where CaD is phosphorylated in order to achieve dynamic actin structures for the disassembly and break down of old actin fibers and a "CaD-zone", where CaD is not phosphorylated and has a high affinity for actin, thereby stabilizing the actin-cytoskeleton for proper lamellipodia formation (Fig. 4.4). 

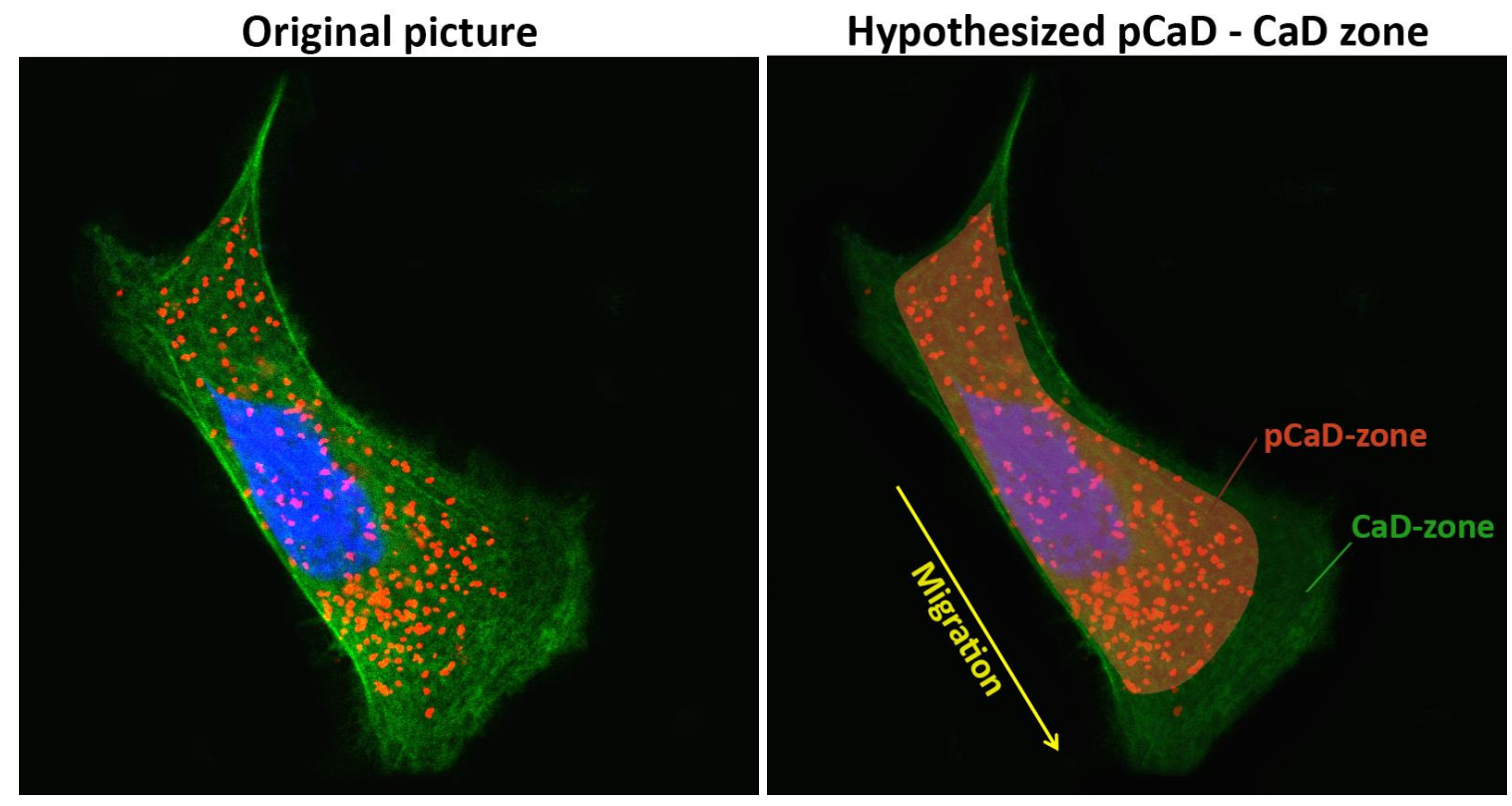

Fig. 4.4: Sub-cellular interaction pattern of LPXN and pCaD during cell migration. The interaction pattern of LPXN and pCaD during migration of PCa cells is generating two zones. A sub-cellular zone, where $\mathrm{CaD}$ is phosphorylated (pCaD-zone) in order to achieve dynamic actin structures and a "CaD-zone" at the leading edge of the migrating cell. In the "CaD-zone" $\mathrm{CaD}$ is present in an unphosphorylated state showing high affinity for actin and thereby stabilizing the actin-cytoskeleton for proper assembly of new actin-cytoskeletal structures.

However, how LPXN is recruited to pCaD-zones and excluded from CaD-zones in lamellipodia and how LPXN recruits ERK to places of pre-dynamic cytoskeletal structures is unclear and requires further investigations. Also the stimuli in that response LPXN might be recruited to these places remains an open question. Localization of LPXN itself to these places could involve its phosphorylation, which was shown to be responsible for sub-cellular localization, e.g. for focal adhesion targeting upon stimulation of the gastrin-releasing peptide receptor (GRPr) (Chen, Kroog 2010). As mentioned above, although $\mathrm{CaD}$ is able to modulate actin-cytoskeleton stability it was reported to bind to intermediate filaments, leaving the possibility that LPXN might additionally affect cell-cell or cell-matrix adhesion through this mechanism. 


\subsection{The role of integrin family proteins in cell adhesion}

\subsubsection{Integrin signaling}

Cell migration affects a variety of cellular processes during development, immune response or tumor metastasis. Usually cell migration starts by sending out new protrusions at the leading edge of the cell. The formation of new adhesions at these protrusions is of major importance to link the actin-cytoskeleton to the substratum to enable cell movement. Integrins are essential adhesion proteins for the connection of the extracellular and intracellular environment and are also crucial for signal transduction. This protein family consists of transmembrane receptors that form heterodimers composed of $\alpha$ - and $\beta$-subunits. Today in mammals there are $18 \alpha$ - and 8 $\beta$-subunits known that, depending on their composition, define the specificity for ligands in the extracellular matrix like collagens, fibronectins or laminins (Hynes 2002b). Integrin activation is regulating the affinity for the corresponding ligands through inside-out signaling, where intracellular activators, e.g. talin or kindlins, bind to the cytoplasmic tail of $\beta$ integrins leading to conformational changes that increase ligand affinity. Therefore, integrin activation via inside-out signaling keeps the strength of connections between integrins and the ECM, required for proper cell migration (Fig. 4.5). Activated integrins can then act as cell surface receptors. Upon ligand binding to their extracellular domain, the integrins form clusters at the plasma membrane. The intracellular signals that are generated (outside-in signaling) lead to the formation of a large protein complex, the focal adhesion (FA) (Zaidel-Bar et al. 2007). Adapter proteins of these multi-protein FA complexes, like the paxillin protein family, allow the assembly of actin filaments and link integrins to the actin cytoskeleton (Giancotti, Ruoslahti 1999). The generation of this linkage is a fundamental step for the regulation of cell shape changes at the leading or trailing edges during cell migration. Thus, integrin-mediated adhesion is a critical regulator of cell migration. 


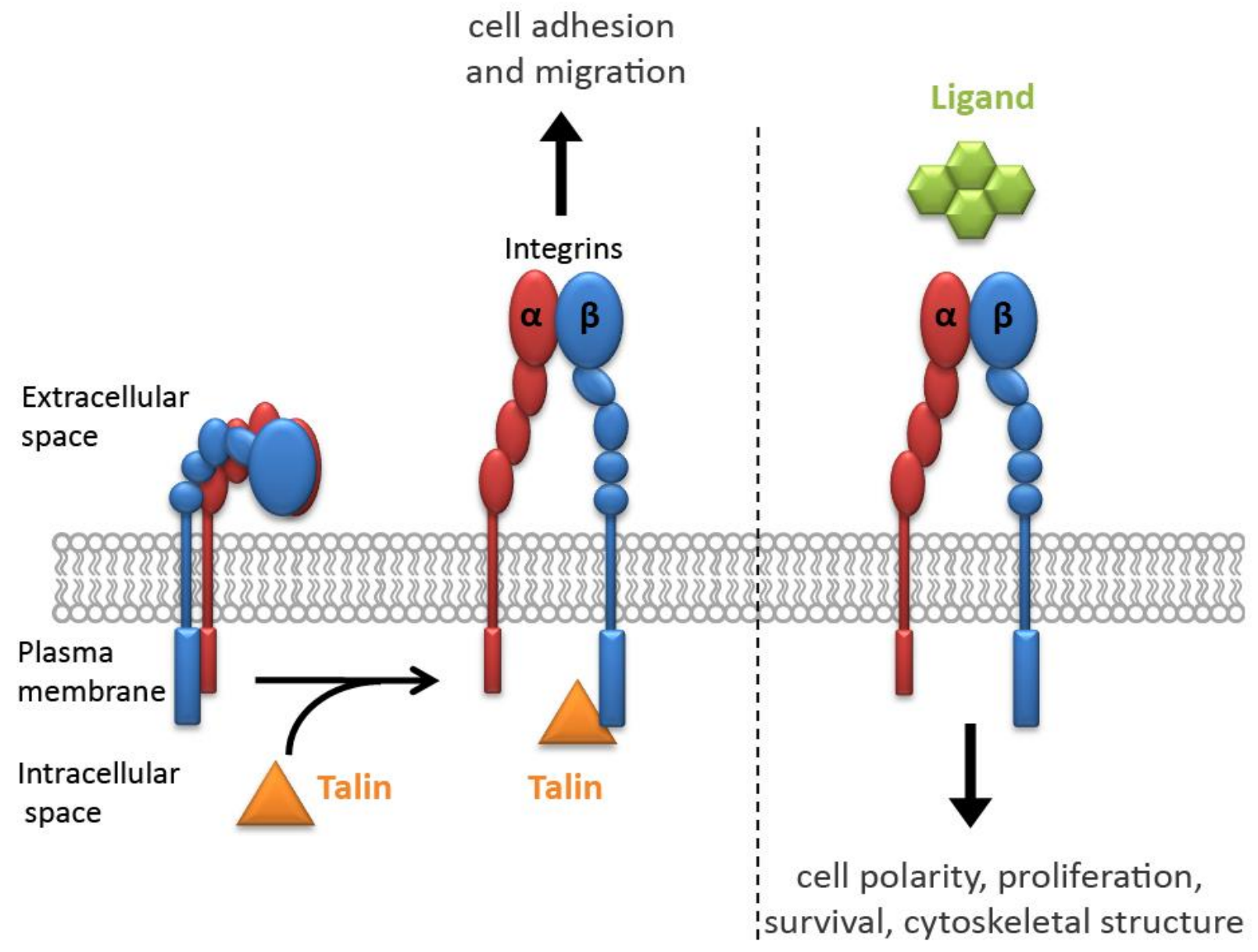

\section{Inside-out signaling}

\section{Outside-in signaling}

Fig. 4.5: Scheme of bidirectional integrin signaling. Integrins can signal in two directions leading to different cellular responses. During inside-out signaling integrins are activated by intracellular activators, e.g. talin, which bind to the cytoplasmic tail of $\beta$ integrins. Subsequent conformational changes increase ligand affinity and contribute to cell adhesion and migration. Ligand binding triggers integrin clustering and formation of a multiprotein complex that forms the focal adhesion. This outside-in signaling will affect cell polarity, proliferation, survival and cytoskeletal structure. Scheme was taken and modified from Shattil et al. 2010.

\subsubsection{Integrins and their implications in radio- and chemo-resistance}

A number of human diseases including immunodeficiency disorders and cancer are linked to altered integrin-mediated adhesion and migration (Allende et al. 2000; Ghevaert et al. 2008; Ruzzi et al. 1997). Additionally, deregulation of integrin expression is often linked to poor patient outcome. For prostate cancer treatment increased $\alpha 3 \beta 1$ levels were shown to be associated with tumor recurrence after radical prostatectomy (Pontes-Junior et al. 2010). Likewise, $\beta 3$ integrin expression correlated with the clinical 
outcome of cervical cancer patients, who had undergone radiotherapy (Gruber et al. 2005). During cancer progression the regulation of integrin-mediated cell adhesion has to be tightly regulated. Whereas, adhesion needs to be reduced in order to leave the primary tumor and enter the circulation for extravasation and metastasis formation the adhesion, e.g. to the endothelium, needs to be reestablished. All these modulations have to happen, while avoiding defensive mechanisms like anoikis. A mechanism that shows how integrin-mediated adhesion regulates migration was suggested by Cox et al., who showed that binding of the $\alpha 5 \beta 1$ heterodimer to its ligand fibronectin (FN) stopped cell migration by inhibition of cell polarization and protrusion through the Rho family of GTPases (Cox et al. 2001). In addition, the adhesion to FN was shown to offer a survival benefit for human myeloma cells (8226) after exposure to the cytotoxic drugs doxorubicin and/or melphalan (Damiano et al. 1999). Moreover, a significant increase in $\alpha 4$-mediated cell adhesion was detected in these cells. This phenomenon was observed in a variety of tumors in chemo- as well as radiation therapy and was termed cell adhesion-mediated drug- (CAM-DR) or radio-resistance (CAM-RR), respectively. ECM adhesion and the constitutive activation of AKT and MAP kinase pathways are common mechanisms that correlate with the acquisition of radioresistance of tumor cells (Cordes, Meineke 2003; Kraus et al. 2002; Sandfort et al. 2007). Recently, the $\beta 1$ integrin, which is involved in several signaling pathways that control proliferation, apoptosis and survival, was shown to be associated with resistance to several chemotherapeutics (Hodkinson et al. 2006; Sethi et al. 1999). Moreover, $\beta 1$ integrin was upregulated in the two lung cancer cell lines A549 and SKMES1 in response to ionizing radiation (Cordes et al. 2002). Another study revealed that $\beta 1$ integrin-stimulated tyrosine kinase activation results in a microenvironment that inhibits chemotherapy-induced apoptosis (Sethi et al. 1999). In addition, adhesion of myeloma cells to FN was observed to be implicated in drug resistance. FN-adhesion leads to an accumulation of these cells in G1 phase, which was quickly released upon adhesion disruption (Hazlehurst et al. 2000). Taken together, it is not surprising that new therapy options are targeting adhesion molecules to reduce radioresistance (Cordes, Park 2007; Kiziltepe et al. 2012; Ni et al. 2013).

The sequence of events that follows ionizing irradiation to acquire resistance is a great challenge in therapeutic research and is incompletely understood. Certainly, focal adhesion-associated proteins are playing an essential role in this process. A well-known feature of resistance mechanisms is the induction of survival and apoptosis-related genes such as those of the Bcl-2 family. The adhesion of Chinese hamster ovary cells to 
FN through $\alpha 5 \beta 1$ integrin was shown to induce Bcl-2 expression and thereby suppressing apoptosis (Paoli et al. 2013). The process that is facilitating anchorageindependent growth of cells exhibiting malignant potential is known as anoikis. Thus, it was not surprising that Bcl-xl, a member of the Bcl-2 anti-apoptotic protein family, was induced in murine prostatic tumor tissue upon ionizing radiation (Zhu et al. 2014). Paxillin family proteins have already been observed to be involved in resistance mechanisms of tumor cells. Paxillin phosphorylation was linked to an increase in the expression of the anti-apoptotic protein Bcl-2. Moreover, paxillin phosphorylation was correlating with resistance to cisplatin treatment of non-small cell lung cancer (Wu et al. 2014). Interestingly, ionizing irradiation induced phosphorylation of paxillin and p130Cas in response to $\beta 1$ integrin activation and resulted in pro-survival effects in GD25 mouse fibroblasts (Seidler et al. 2005).

\subsubsection{LPXN influences radio-resistance through regulation of integrin expression}

As adapter proteins at focal adhesion sites and their recruitment to $\beta$-integrin tails, paxillin protein family members take part in signal transduction and the acquisition of radioresistance. In the present study we showed that $\beta 1$ integrin expression is associated with LPXN expression and that both, LPXN as well as $\beta 1$ integrin expression were induced by ionizing irradiation. Downregulation of LPXN by RNA interference clearly sensitized the PCa cells to irradiation, which was also observed when $\beta 1$ integrin activity was blocked by the AIIB2 inhibitory antibody. In addition, the irradiationinduced expression of $\beta 1$ integrin was prevented by LPXN knockdown, suggesting that LPXN is involved in the acquisition of radioresistance and might act upstream of $\beta 1$ integrin in irradiation-responsive signaling of the PCa cell lines PC-3, DU145 and LNCaP. Cordes et al. observed that after irradiation of human lung tumor cells the expression of $\beta 1$ integrin was increased, which did also enhance cell adhesion. In the following studies they were detecting a prolongation and increased accumulation of cells in G2 phase after exposure to ionizing irradiation (Cordes et al. 2002; Cordes, van Beuningen 2004). However, the induction of adhesion-associated genes by ionizing irradiation seems to be a common feature not only of tumor cells (Nübel et al. 2004, Hallahan, Virudachalam 1997). Interestingly, the expression and phosphorylation of the focal adhesion- 
associated proteins p130cas, FAK and paxillin were shown to be induced by ionizing irradiation in A549 human lung carcinoma cells (Beinke et al. 2003). These results indicate that these genes are likely to play a role in the cellular response to ionizing irradiation. Most likely the microenvironment of the cells is altered by irradiation, which might explain why primarily adhesion-associated genes are affected by this process.

LPXN might exert its function in radioresistance by two possible mechanisms. The first mechanism is suggesting LPXN to influence $\beta 1$ integrin expression directly, leading to a mediation of the cellular responses downstream of $\beta 1$ integrin. This mechanism could further involve ERK1/2, which was already shown to be influenced by LPXN (Kaulfuss 2006) to act as a regulator of the DNA binding ability of the transcription factor SP1 (Merchant et al. 1999; Milanini-Mongiat et al. 2002) (Fig. 4.6). This way is further strengthened by the fact that we could observe an upregulation of Sp1 activity by overexpression of LPXN. However, LPXN knockdown also resulted in an upregulation of Sp1 activity that was not significant. This contradiction could be based on the fact that the downregulation of LPXN was not strong enough and that remaining LPXN levels were sufficient to induce $\mathrm{Sp} 1$ activity. Nevertheless, our results are suggesting a mechanism for the LPXN-mediated expression of $\beta 1$ integrin that could have influence on cell adhesion and promote the acquisition of radioresistance. In addition to our studies, $\mathrm{Sp} 1$ was also shown to bind to several integrin promoters, like the human $\alpha 6$ subunit gene in response to the ECM protein laminin, the $\alpha 2$ subunit gene or the $\alpha 5$ subunit gene in breast tumor cells and rabbit corneal epithelial cells (Gingras et al. 2003; Zutter et al. 1997; Sisci et al. 2010; Gaudreault et al. 2007). Interestingly, it was shown for the PCa cell lines PC-3 and LNCaP that Sp1 is necessary for basal promoter activity of the $\alpha 6$ integrin (Onishi et al. 2001). 


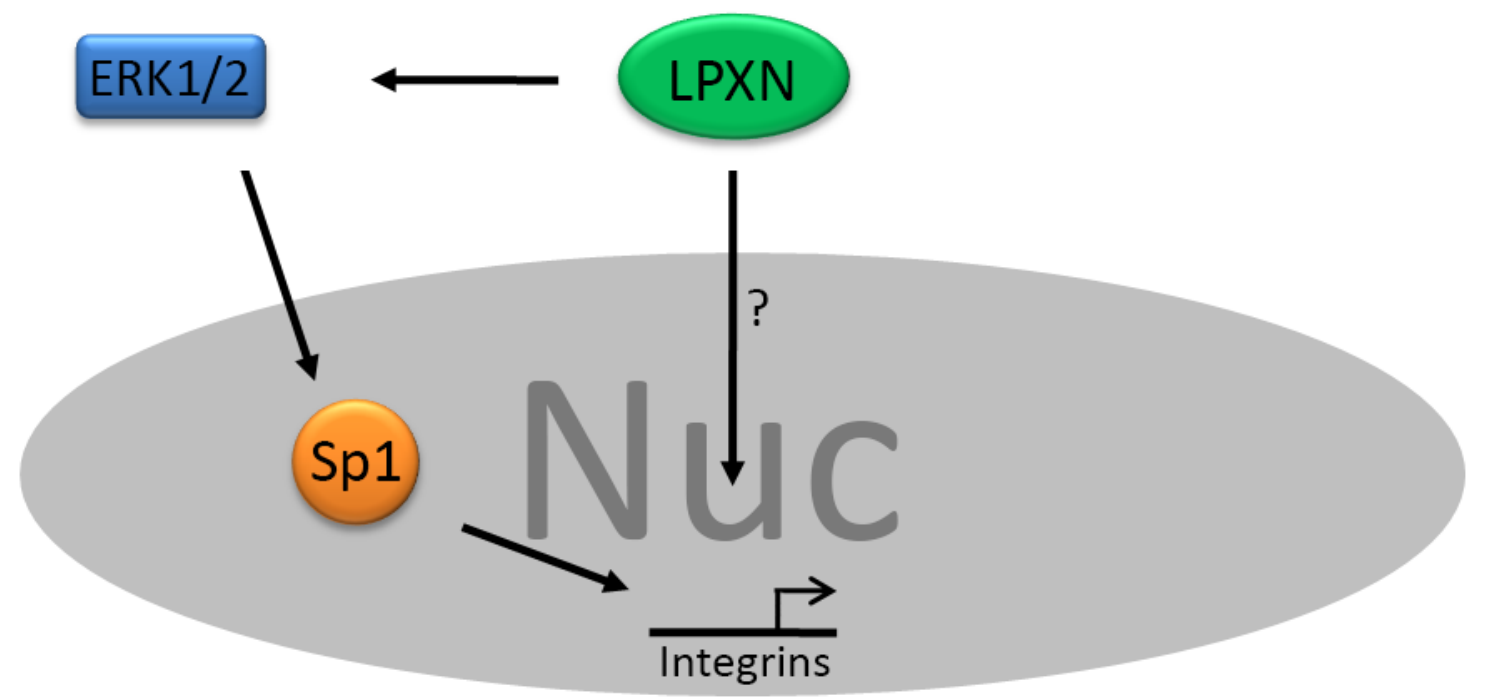

Fig. 4.6: Overview of how LPXN might regulate integrin expression in the acquisition of radioresistance. LPXN might exert its influence on $\beta 1$ integrin expression directly or via ERK1/2 and Sp1. LPXN knockdown was reported to reduce ERK1/2 activity. ERK1/2 in turn is able to modulate DNAbinding activity of $\mathrm{Sp} 1$, which was reported to bind to several integrin promoters. The depicted mechanism displays a possible way of LPXN-mediated integrin expression. Nuc=Nucleus; Sp1 = Specificity protein 1; ERK1/2 = Extracellular signal-regulated kinase 1/2. Modified from Gauldreault et al. 2007 and Merchant et al. 1999.

According to what is known from the literature LPXN could also mediate radioresistance by interaction with its binding partners FAK or SRC, which are located downstream in the integrin-signaling pathway and have already been reported to interact with paxillin. Phosphorylation of FAK upon $\beta 1$ integrin inhibition resulted in a dissociation of the FAK/cortactin protein complex, which reduced JNK signaling and induced cell rounding and radiosensitization (Eke et al. 2012). Possibly, LPXN might influence the phosphorylation status of FAK and control downstream signaling. A depletion of FAK from mouse squamous cell carcinoma (SCC) was associated with increased radioresistance in advanced SCC. Re-expression of FAK in these cells induced upregulation of p53 target genes involved in DNA repair like p21, in response to ionizing radiation, suggesting a role of FAK in radioresistance (Graham et al. 2011). FAK has also been described to mediate drug resistance, because the circumvention of docetaxelinduced cleavage of FAK was suggested to contribute to the resistance of taxaneresistant ovarian cancer cell lines. Reduction of FAK expression by RNAi-mediated knockdown promoted sensitization of these cells to docetaxel (Halder et al. 2005). A summary of the radioresistance mechanisms that are based on the findings of this study 
as well as other possible mechanisms of how paxillin proteins might regulate radioresistance is displayed in figure 4.7.

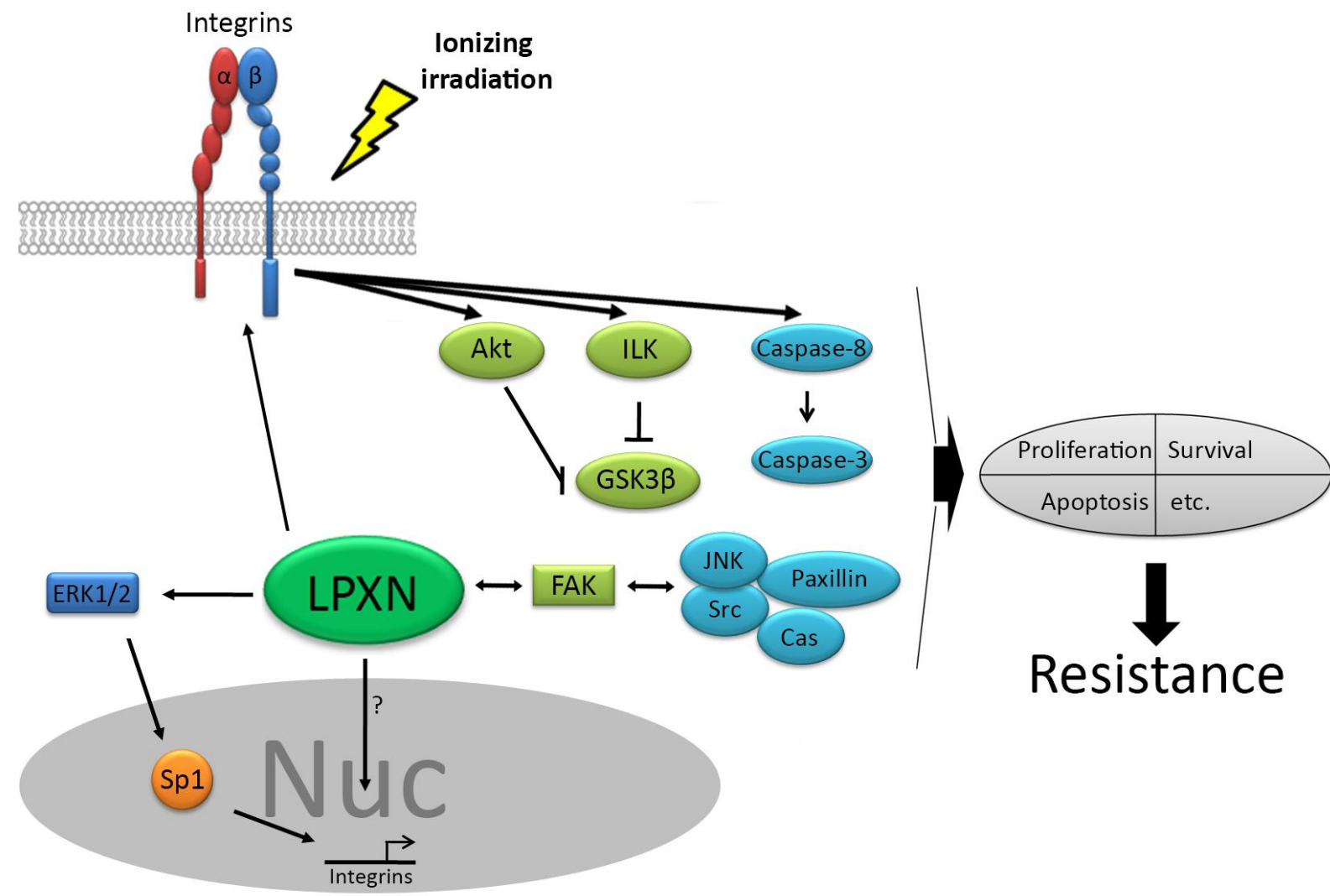

Fig. 4.7: Schematic overview of signaling pathways associated with $\beta 1$ integrin after exposure of cells to ionizing irradiation and how LPXN might interfere in these pathways to modulate radioresistance. LPXN might affect several mechanisms to mediate radioresistance. It could control ERK $1 / 2$ activity, which controls Sp1 DNA-binding to regulate integrin expression. $\beta 1$ integrin signaling involves several pathways including caspases, Akt, and GSK3 $\beta$ to modulate cellular responses like apoptosis, survival or proliferation that play a role in the acquisition of radioresistance. LPXN might exert its radio-sensitizing function in several ways. It might directly act on $\beta 1$ integrin expression via association with transcription factors like $\mathrm{Sp} 1$ or it might mediate radioresistance by interaction with its binding partners FAK or SRC. Binding partners that have also been reported to associate with paxillin. Nuc=Nucleus; ILK = Integrin-linked kinase; GSK3 $\beta$ = Glycogensynthase-kinase $3 \beta$; JNK = c-Jun-N-terminal kinase; Src = sarcoma kinase; Cas = Crk-associated substrate. Scheme was taken and modified from Cordes, Park 2007 and Merchant et al. 1999.

Cell adhesion displays an essential process for normal cell function. Its importance becomes illustrated by its deregulation resulting in tumor development or cell death. As mentioned earlier cell adhesion has also influence on the acquisition of resistance to radiation. Besides radioresistance cell adhesion is also affecting a phenomenon known 
as anoikis, a programmed cell death that is autonomously initiated upon loss of adhesion to the ECM. Anoikis is a protective mechanism of the organism to prevent adhesionindependent growth that might end in colonization of distant organs. Tumor cells need to circumvent this programmed cell death upon ECM detachment to survive during their life-threatening journey. Integrin composition and expression severely affect anoikis and contribute to anoikis resistance. In the present study LPXN was shown to regulate $\beta 1$ integrin expression. Thus, LPXN might not only mediate radioresistance but could also affect the resistance to anoikis. Although this mechanism was not studied in the present thesis, previous studies in our research group showed that downregulation of LPXN in PC-3 and DU145 cells resulted in a sensitization of these cells to anoikis (Hitzing, 2010). Therefore, LPXN could be assumed to contribute to anoikis resistance by either regulating integrin expression or interfere with downstream effector protein of the integrin signaling pathway. This hypothesis could explain the resistance to anoikis of PC3 and DU145 cells. However, these cells have been isolated from bone metastasis and have been through the process of metastasis including epithelial to mesenchymal transition (EMT), invasion of the surrounding tissue, entering the lymph or blood stream and exiting the circulation at a distant site. This is a sequence of events that requires modulation of cell adhesion throughout the whole process. Therefore, the anoikis-resistance of these cells might not be critically for their survival anymore and might be a beneficial remain from the pre-metastatic stages.

Taken together, focal adhesion-associated proteins have an important role in the acquisition of resistance mechanisms by governing cell adhesion and cell adhesioninduced signaling. Hence, LPXN displays a fundamental part to regulate a complex interplay of an enormous array of proteins and contributes to radioresistance of $\mathrm{PCa}$ cells. This study identified LPXN to regulate the expression of $\beta 1$ integrin through Sp1. By this mechanism LPXN was suggested to mediate the adhesion and radioresistance of PCa cells. However, if this mechanism involves other proteins and what underlies the irradiation-induced expression of LPXN requires further investigations and might also elucidate the acquisition of anoikis-resistance and contribute to the development of new therapeutic strategies.

From the findings of this study the therapy of LPXN positive tumors using irradiation might quickly result in an acquisition of radioresistance and would reduce therapy effectiveness. Therefore, LPXN could be used as a biomarker for PCas that are likely to develop radioresistance and would contribute to individualize PCa therapy for 
maximum outcome. To sensitize PCa cells to radiation LPXN itself could display a putative target to improve the effectiveness of radiotherapy of PCa. How exactly LPXN can be targeted requires further research. In addition, the investigation of integrinmediated cell adhesion mechanism of PCa could reveal additional targets that could imply new therapeutic targets in PCa therapy.

\subsection{How does LPXN regulate the expression of the adhesion protein p120-catenin?}

\subsubsection{The implications of the cell adhesion molecule p120CTN in LPXN-mediated PCa progression}

Cell adhesion is a fundamental process for the growth, migration and differentiation of cells. Cell adhesion molecules (CAMs) are proteins important for cell-cell and cell-matrix interaction (Cohen et al. 1997). Basically, CAMs are categorized into four groups, the immune globulins, selectins, intergrins and cadherines (Okegawa et al. 2002). Defects in cadherin expression are linked to tumor progression and are known to function as a tumor suppressor during cell invasion and metastasis (Herzig et al. 2007; Perl et al. 1998; Jeanes et al. 2008). Cadherins do not possess an intrinsic catalytic activity. Therefore, their cytoplasmic domains associate with the group of catenin proteins, which facilitate signal transduction and link the cadherins to the actin cytoskeleton (Mege et al. 2006; Behrens 1999). The interaction of cadherins with catenins is essential for the formation of the adhesion complex and important for proper cell adhesion and the maintenance of the tissue architecture. Disruption of these contacts will end up in an aggressive and invasive growth of tumor cells (Wijnhoven et al. 2000). The adhesion complex consists of $\beta$-catenin, $\alpha$-catenin, $\gamma$-catenin and p120-catenin (p120CTN). For $\beta$ catenin a tumor promoting function was described and also an abnormal expression of p120CTN was detected in different tumor entities (Kallakury et al. 2001; Chetty et al. 2008; Perez-Moreno et al. 2008; Liu et al. 2009). Predominantly in colorectal cancer a mutation in the APC gene, a component of the Wnt signaling pathway, results in an impaired degradation of $\beta$-catenin and subsequent accumulation in the nucleus (Schneikert, Behrens 2007). In the nucleus $\beta$-catenin is binding to the transcription complex of TCF/LEF ( $\mathrm{t}$-cell-factor/lymphoid enhancer factor) leading to the activation of 
Wnt- $\beta$-catenin target genes implicated in cell proliferation and cell migration such as the group of matrix metalloproteinases or cyclins (Fig. 4.8) (Friedrich, Kullmann 2003).

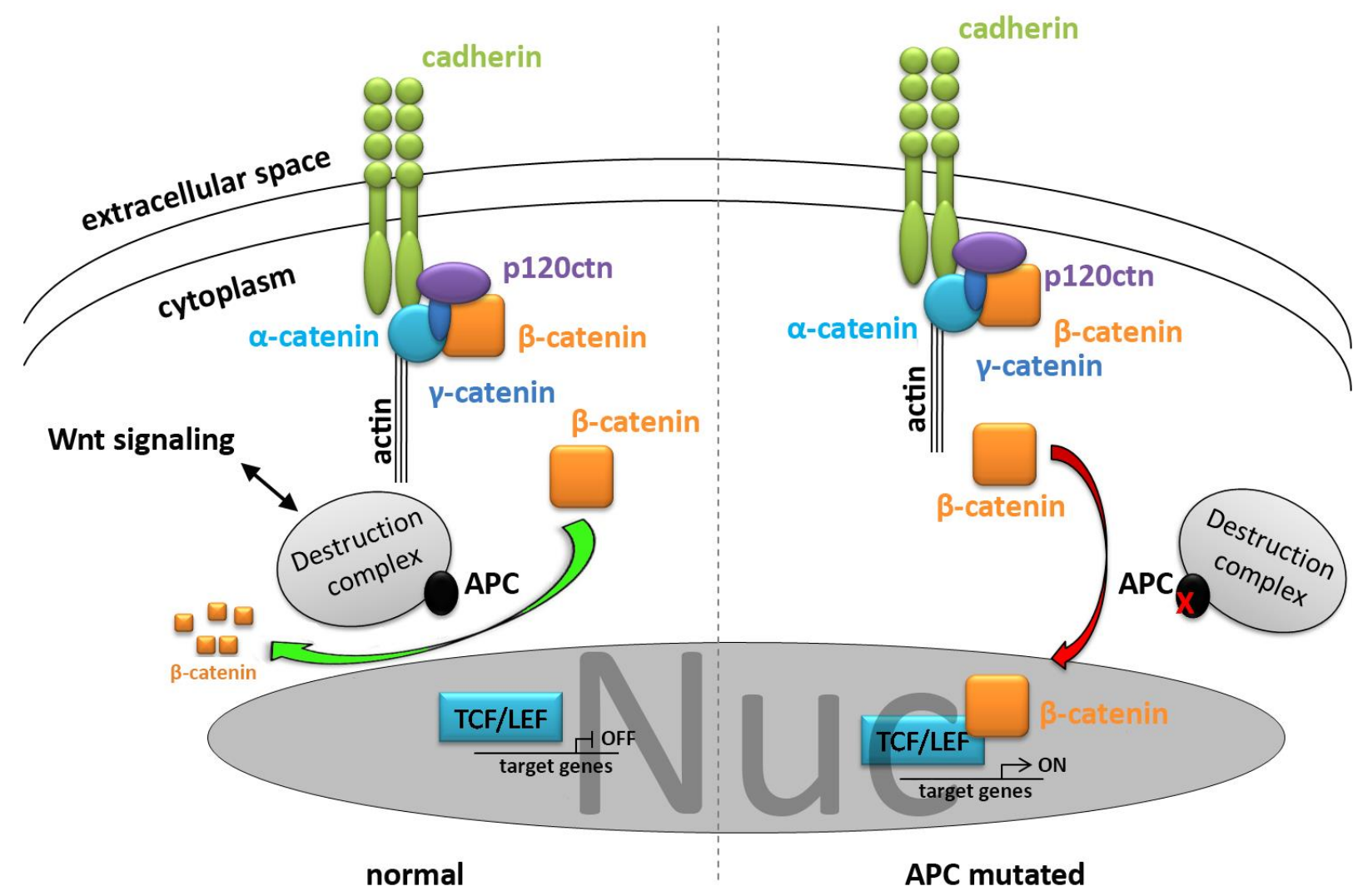

Fig. 4.8: Scheme of APC-mediated degradation of $\boldsymbol{\beta}$-catenin. Under normal conditions the adhesion complex is stabilized and connects the cadherins to the actin cytoskeleton. Cytoplasmic $\beta$-catenin is primed for degradation via the proteasome (ubiquitin) by the destruction complex, containing the adenomatous polyposis coli (APC) protein. APC is an important component of the Wnt signaling pathway. Upon APC mutation, cytoplasmic $\beta$-catenin degradation is impaired and subsequently accumulates in the cytoplasm and the nucleus. Once in the nucleus $\beta$-catenin associates with TCF/LEF to activate target genes. Nuc=Nucleus. Modified according to Morin et al. 1997.

The catenin $\mathrm{p} 120$, which is encoded by the CTNND1 gene, is part of the p120 armadillo protein family. Originally, in 1989 p120CTN was described as a substrate of tyrosine kinases (Reynolds et al. 1989). New evidence is suggesting p120CTN to have multiple functions. On the one hand it takes part in the dynamic regulation of the actin cytoskeleton and cadherin transport to the membrane. On the other hand it binds to the cytoplasmic domain of cadherins at the plasma membrane and stabilizes the cell-cell adhesion complexes. A nuclear localization of p120CTN has also been described, but except for its association with the transcription factor kaiso, the nuclear function of p120CTN remains largely unsolved. As mentioned above, changes in expression levels of 
p120ctn are detected in a number of tumor types. A disturbed expression of p120CTN caused a destabilization of the adhesion complexes and a degradation of cadherins in the colorectal cell line SW48 (Davis et al. 2003). In the majority of carcinomas, which derive from epithelial cells, the dysfunction, degradation or loss of cadherin expression is a common finding (Onder et al. 2008; Birchmeier, Behrens 1994; Thiery 2002; Yap 1998). The loss of p120CTN was associated with metastatic progression and anoikis resistance in breast cancer cells and a complete p120CTN knockout results in tumor development in mice (Schackmann, Ron C J et al. 2013; Stairs et al. 2011). Accordingly, these studies establish p120CTN as a tumor suppressor gene.

The transcriptional regulation of the p120CTN gene is incompletely defined. In nonsmall cell lung cancer (NSCLC) the Sp1 transcriptional factor was shown to act as a transcriptional repressor of p120CTN. Furthermore, Sp1 was suggested to be a possible mediator of p120CTN repression by the proto-oncogene cellular-CT10 Regulator of Kinase (c-Crk). However, this hypothesis was not confirmed in this study (Mortazavi et al. 2011). In addition, the same research group showed that p120CTN is transcriptionally downregulated by the forkhead box C2 (FOXC2) transcription factor (Mortazavi et al. 2010).

Recently, our lab could identify p120CTN as a mediator of LPXN-induced invasion and progression of PCa (Kaulfuss et al. 2009). In our studies p120CTN expression was negatively correlated with LPXN expression. Downregulation of LPXN resulted in increased p120CTN expression and a membranous localization of $\beta$-catenin. In contrast, overexpression of LPXN caused a decrease in p120CTN expression and subsequent destabilization of the adhesion complexes. Following adhesion complex destabilization, $\beta$-catenin was accumulating in the nucleus leading to activation of the $\beta$-catenin target gene matrix metalloprotease-7 (MMP-7) important for degradation of the extracellular matrix (Fig. 4.9). However, how LPXN might regulate the expression of p120CTN is unknown. 


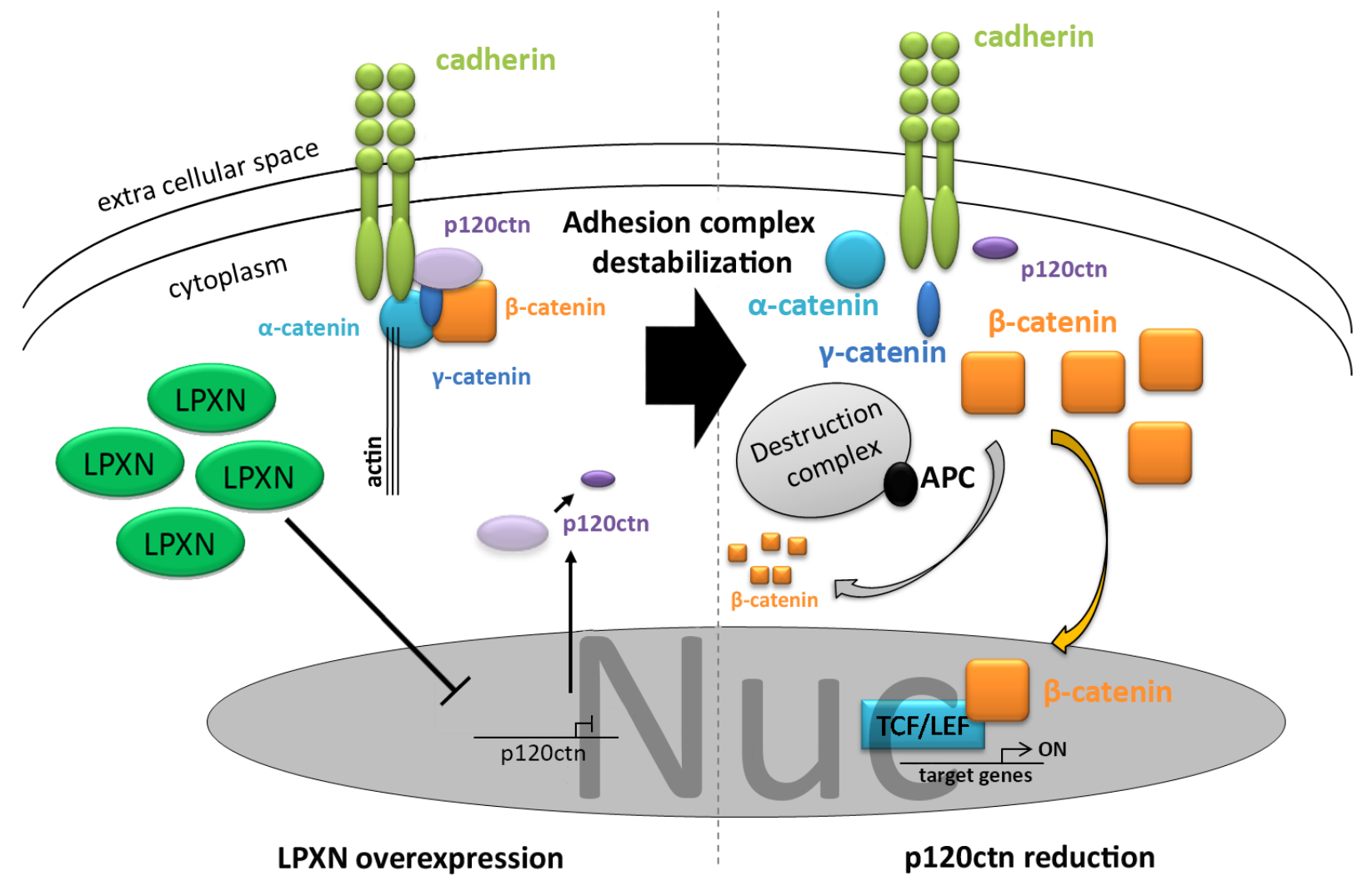

Fig. 4.9: Overview how LPXN overexpression of PCa cells leads to adhesion complex destabilization and nuclear accumulation of $\boldsymbol{\beta}$-catenin. Under normal conditions the adhesion complex composed of $\beta$ catenin, $\gamma$-catenin, $\alpha$-catenin and p120-catenin is formed and stabilized at the cytoplasmic tail of the cadherin and linked to the actin cytoskeleton. In PC-3 and DU145 cells LPXN is overexpressed and causes p120ctn expression levels to decrease. Upon p120CTN reduction the adhesion complex is destabilized. Subsequently, $\beta$-catenin is released from the adhesion complex to the cytoplasm, where it is primed for degradation via the destruction complex containing APC. However, $\beta$-catenin will also accumulate in the nucleus and associate with TCF/LEF transcription factors leading to $\beta$-catenin target gene transcription. Modified according to Morin et al. 1997

\subsubsection{The nuclear function of paxillin family proteins}

Recent investigations have shown that paxillin and its protein family members take functions beyond their main roles as molecular adapter proteins located at adhesion plaques. They were described to mediate extra- as well as intra-nuclear signaling, the latter being poorly understood. Within the nucleus, proteins of the paxillin family were described to act as steroid hormone nuclear receptor coactivators. In our studies, we could identify LPXN to play a role in adhesion and invasion of prostate carcinoma cells by coactivation of the androgen receptor (Kaulfuss et al. 2008). In addition, LPXN was 
shown to form a complex with the transcription factor, serum response factor (SRF), in human and mouse vascular smooth muscle cells, driving SRF-responsive gene expression (Sundberg-Smith et al. 2008). Likewise, paxillin was shown to be involved in the progression of castration-sensitive to castration-resistant PCa by association with the androgen receptor (AR) (Sen et al. 2012). For ARA55 association with the androgen receptor, the glucocorticoid receptor and the peroxisome proliferator-activated receptor gamma (PPAR $\gamma$ ) was shown (Yang et al. 2000; Fujimoto et al. 1999; Heitzer, Defranco 2006). Thus, paxillin family proteins clearly have a nuclear function. However, it is not much known about the mechanism of nuclear/cytoplasmic shuttling and only a few suggestions have been proposed. Paxillin proteins do not possess a nuclear localization signal (NLS) and might therefore enter the nucleus with the help of other proteins. The nuclear import of LPXN has not been elucidated today. For paxillin, phosphorylation of the LD4 motif was described to regulate nuclear localization, which was correlating with cell proliferation (Dong et al. 2009). In contrast, nuclear accumulation of ARA55, another paxillin family protein, turned out to be regulated by reactive oxygen species (ROS), which is why it was originally named hypoxia-inducible clone 5 (Hic-5) (Shibanuma et al. 2003). The nuclear export of paxillin family proteins is facilitated by a leucine-rich nuclear export signal (NES) (Wang, Gilmore 2003). Especially for LPXN our research group identified NES in the LD3 and LD4 motifs in the N-terminal region of the protein (Kaulfuss et al. 2008).

Undoubtedly, paxillin family proteins are able to shuttle between the cytoplasm and the nucleus. Localization to the nucleus and their association and co-activation of nuclear steroid hormone receptors like the androgen receptor or glucocorticoid receptor clearly grants them nuclear functions. A direct DNA binding was only described for ARA55 (Nishiya et al. 1998). Neither paxillin nor LPXN were shown to bind directly to DNA sequences.

In the present study the DNA binding of LPXN to the p120CTN promoter region was analyzed to elucidate the negative correlation of both proteins. Fragmentation of the p120CTN promoter region and subsequent analysis indeed identified a LPXN responsive region. Using luciferase reporter gene assays the region from $240 \mathrm{bp}$ upstream to $349 \mathrm{bp}$ downstream relative to the transcriptional start site was identified to show the highest activity upon LPXN knockdown in comparison to control transfected cells. Larger promoter fragments also showed increased activity but this increase was not caused by LPXN, as it was also detectable in control-transfected cells. Further investigation of this 
LPXN-responsive region using EMSA, showed no direct binding of LPXN to the p120CTN promoter region. Even an enrichment of an EGFP-LPXN fusion protein in the nucleus, did not shown any differences in the band pattern compared to EGFP control transfected cells. Also we did neither detect binding of the Sp1 transcription factor to the proposed binding sites in the p120CTN promoter region by Mortazavi et al. nor did we observe Sp1 binding to a consensus sequence (Kadonaga et al. 1988). However, with the EMSA we were only able to analyze a small isolated section of the p120CTN promoter region, which is composed of a variety of promoting and repressing sequences (Mortazavi et al. 2010; Mortazavi et al. 2011). Therefore, we focused on the proximal promoter region around the transcriptional start site. Possibly, we cannot exclude that we might have missed other important regions with this decision.

Since a negative correlation of LPXN and p120CTN expression was reported in previous studies of our research group, it is likely that LPXN is involved in the regulation of p120CTN expression. Certainly a direct binding of LPXN to the proximal p120CTN promoter region could not be demonstrated. Apart from this, only for the paxillin protein family member, ARA55, a direct, zinc-dependent binding to an adenine-rich DNA sequence via its LIM domains was reported (Nishiya et al. 1998). Usually LIM domains were thought to be restricted to protein-protein interactions, but as mentioned earlier, the LIM domains of paxillin family proteins might be potentially DNA binding interfaces. Interestingly, LIM domains were originally discovered in the three transcription factors lin 11, isl-1 and mec-3 and accordingly named after them. These LIM domains are highly conserved residues that form a tandem zinc finger motif. The fact that zinc fingers are important structures in DNA binding proteins such as transcription factors, called the attention that also LIM domain-containing proteins are capable of DNA binding (Kadrmas, Beckerle 2004). ARA55 was the first LIM domain-containing protein that was observed to bind to DNA. In addition, this research group was not successful in discovering a consensus sequence for ARA55. Rather it was suggested to bind to a poly(A) DNA sequence such as those present in the 3'-end of mRNAs (Nishiya et al. 1998). A similar mechanism was proposed for paxillin, which interacts with the poly(A)binding protein and thereby transports certain mRNAs to the leading edge of migrating cells (Woods et al. 2002). Interestingly, there seem to be certain types of protein-DNA complexes that are not stable when subjected to conventional gel shift assays (von Kries, J P et al. 1991; Romig et al. 1992). The authors suggested that these proteins do not recognize a specific DNA sequence but the secondary structure within those sequences. 
In contrast, these secondary DNA-structure-binding proteins were successfully precipitated in a DNA binding protein blot assay. Thus, LPXN might also bind to a secondary DNA structure rather than to a specific DNA sequence and this might account for the negative EMSA results obtained. On the other hand DNA binding of LPXN was not detected in a DNA cellulose pulldown in previous experiments (Dierks, 2011). Therefore, DNA binding of LPXN might be restricted to sequences that escaped our analyses or the binding to secondary DNA structures required other conditions than those used in our binding assays. Further analysis of the human p120CTN promoter showed the presence of putative steroid hormone receptor binding sites, which are known to be activated by LPXN, but remain inconclusive. Possibly, LPXN is not directly binding to DNA sequences but executes its regulatory function through other transcription factors that negatively influence p120CTN expression. Hence, LPXN might influence p120CTN expression through other, yet unknown mechanisms.

\subsection{The physiological role of LPXN}

Paxillin proteins are effectors of integrin signals and play major roles in cell adhesion and signal transduction. Their predominant localization at focal adhesion sites and domain structure of multiple protein-protein interaction interfaces grants them their primary role as molecular adapter proteins, which orchestrate the complex dynamics of signaling and structural proteins.

Whether paxillin family proteins can compensate for the loss of other protein family members is not completely known. The role of paxillin and leupaxin in cell adhesion and spreading of MDA-MB231 breast cancer cells found that both proteins are similar in focal adhesion targeting and tyrosine phosphorylation but each protein has distinct functions (Chen, Kroog 2010). Since paxillin protein family members share a number of interaction partners it is likely that they might compensate for the loss of other paxillin proteins. Nevertheless, there are unique interaction partners for each protein within the paxillin protein family, which suggests that they could modulate each other's functions (Turner et al. 1990; Thomas et al. 1999). However, if paxillin proteins have an inhibitory effect on each other's functions needs to be determined. The fact that paxillin deficiency results in embryonic lethality suggests that neither LPXN, nor ARA55 can substitute the functions of paxillin and further strengthen the hypothesis that each protein has its specific functions. 
The function of LPXN in the organism is not clear and a knockout of LPXN has not been reported. In our research group a mouse model was generated that specifically expressed LPXN under the control of the prostate-specific rat probasin promoter. This leads to an overexpression of LPXN in the prostate and resembles the clinical situation found in approximately $20 \%$ of the PCa patients. Prostate-specific overexpression of LPXN showed that it is not involved in tumor initiation. Further investigations included breeding of these mice to the TRAMP mouse model (transgenic adenocarcinoma of the mouse prostate), which specifically expresses the simian virus 40 (SV40) large T antigen in the prostate inducing tumor development. Double transgenic TRAMP/LPXN mice showed enhanced tumor progression in comparison to single transgenic TRAMP mice (Kaulfuss et al. 2009), suggesting LPXN to play a role in cell invasion and migration. These results are consistent with the findings that the highly invasive PCa cell lines PC-3 and DU145 show high LPXN expression and that LPXN knockdown leads to reduced migration and invasion of these cells (Kaulfuss et al. 2009).

To understand the physiological role of LPXN a targeted trap of the LPXN gene was generated. Mice carrying the conditional LPXN allele were then bred to mice that express the cre-recombinase under the control of the adenoviral EIIa promoter, which targets cre expression to the early embryo. Like observed for ARA55 knockout mice, $\mathrm{LPXN} /$ mice are viable and fertile, which suggests that LPXNs function is not important during embryonic development. Loss of LPXN function may also be compensated by one of its protein family members. However, a detailed analyses of LPXN-/-mice concerning tissue structure and especially the immune system is needed. Since LPXN was initially reported to be expressed in motile cells of hematopoietic origin analyses of these tissues seems promising to uncover a phenotype of these mice.

Paxillin knockout results in embryonic lethality at E9.5. Until this stage paxillin was expressed in extraembryonic structures such as parietal endoderm, ectoplacental cone and extraembryonic mesodermal structures like the chorion, amnion and allantois (Hagel et al. 2002). Thus paxillin is essential for proper embryonic development. Noteworthy, the phenotype of the paxillin knockout mouse model is closely reflecting the phenotype of fibronectin $\%$ mice (George et al. 1993; Watt, Hodivala 1994). Fibronectin is a major component of the extracellular matrix and ligand of a number of integrin heterodimers, which suggests paxillin to be an effector of integrin signaling during early embryonic development. This fact is further strengthened by paxillin null embryonic stem cells that display delayed spreading on fibronectin and laminin and 
reduced phosphorylation of attachment-dependent FAK phosphorylation (Wade et al. 2002).

ARA55 knockout mice are viable and fertile and do not show any abnormalities in development and tissue homeostasis. However, ARA55 may have a role in vascular remodeling because recovery of injured arteries was delayed in ARA55 deficient mice (Kim-Kaneyama et al. 2011). In addition, ARA55 expression is induced by the transforming growth factor- $\beta$ (TGF $\beta$ ), a well-known promoter of fibrosis, which involves ARA55 in a number of fibrotic disorders such as glomerulosclerosis (Border, Noble 1993).

In conclusion, the LPXN knockout mouse model does not show an obvious phenotype or embryonic lethality. The phenotypes of ARA55 and paxillin knockout mice suggest that each protein has its particular function. Detailed studies of the LPXN knockout mice, especially on cells of hematopoietic origin, are thought to give promising results and are required to contribute to the understanding of LPXNs physiologic function. This knowledge can then be transferred to uncover its pathologic function and help to develop therapeutic strategies.

\subsection{Perspectives}

In the present study LPXN was identified to exert its pro-migratory function on PCa by directly affecting the actin cytoskeleton through modulation of the phosphorylation status of the actin-binding protein CaD. Out of these studies LPXN was released from its classical role as a molecular adapter protein at focal adhesions and hypothesized to recruit the extracellular signal-regulated kinase $1 / 2(\mathrm{ERK} 1 / 2)$ in order to facilitate CaD phosphorylation. Undoubtly, CaD phosphorylation alone is not sufficient for actin cytoskeletal remodeling and migration of PCa cells. Thus, LPXN might facilitate migration by other mechanisms that remain unknown so far. The fixation of migrating cells for microscopic analysis has been a major obstacle in the study of migrating mechanisms that are often spatially and temporally restricted. Therefore, life cell microscopy might display a suitable opportunity to study the LPXN-dependent processes during migration of PCa cells. In addition, in vivo studies should confirm the relevance of LPXN-mediated phosphorylation of CaD through ERK1/2. For this purpose the phosphorylation status of ERK1/2 and CaD of prostate tumors isolated from double transgenic LPXN/TRAMP-mice should be analyzed by western blot. Furthermore, it has 
to be assumed that LPXN has influence on other processes and signaling pathways that promote tumor progression. For instance, LPXN might be implicated in ERK1/2 downstream signaling, since LPXN knockdown was affecting ERK1/2 activity. However, the fact that paxillin family proteins carry functions beyond their original role as molecular adapters suggests that these proteins may be involved in other processes as well. These processes might also have promoting influence on cancer progression and should be subject to further investigations to identify LPXNs function in more detail. In addition, these studies could help to establish LPXN as a putative progression marker and to develop new therapeutic treatment options. Obviously, reduction of LPXN expression would display such a treatment possibility to reduce $\mathrm{CaD}$ phosphorylation, stabilize the actin cytoskeleton and hamper PCa cell migration. Since modern cancer treatment will be more and more individualized for each patient to decrease site effects and yield maximum therapeutic response, further investigations that uncover the physiologic role of LPXN may be the key to understand the pathologic function of LPXN and will also contribute to the development of new therapeutic approaches for PCa therapy. Targeting LPXN overexpression in PCa itself would require siRNA-based treatment approaches. These RNAi techniques have been shown to successfully reduce expression of targeted genes in cancer therapy. However, the main challenge of this method is its efficacy and the delivery of nucleic acids specifically to tumor cells after intravenous application. In addition, many siRNAs display off target gene modulation, which may result in additional site effects. Nevertheless, latest studies that were able to specifically target mutant oncogenic ras but were not affecting wild type ras show promising results and would revolutionize the field of RNAi in cancer therapeutics (Lois et al. 2001). According to the findings from this study and the complexity of PCa, it seems unlikely that LPXN has such strong influence on the tumor progression to be considered as a single target. Rather LPXN should be noticed as a protein that is integrated in a complex network of genes, which need to be included in the development of therapeutic approaches.

In the present study LPXN was emphasized to play a role in the acquisition of radioresistance. LPXN expression was found to be inducible by ionizing irradiation and further shown to regulate the expression of $\beta 1$ integrin. By this mechanism LPXN might affect cell adhesion, which is a common feature of cells after exposure to ionizing irradiation. Knockdown of LPXN resulted in radio-sensitization of PCa cells, a finding that might also be relevant for radiotherapy of e.g. PCa. Resistance mechanisms are 
mostly regulated through the expression of genes that are important for apoptosis or survival. The analysis of these genes (e.g. Bcl-2 family genes) after exposure to ionizing irradiation and their response to LPXN overexpression or knockdown could reveal how LPXN exactly mediates radioresistance. These experiments might also add to the understanding of general radioresistance mechanisms and how to circumvent radioresistance during radiotherapy.

As mentioned above LPXN should not be considered as a pure focal adhesion adapter protein. Especially the nuclear presence and the coactivation of the androgen receptor point towards a broader function of LPXN in a variety of processes. The negative correlation of LPXN and p120CTN expression and their suggested implications in PCa cell invasion have been reported lately (Kaulfuss et al. 2009). The basis for this negative correlation was analyzed in this study. However, a direct binding of LPXN to the promoter region of CTNND1 was not found. Hence, additional studies including chromatin immunoprecipitation (ChIP) have to be conducted to clarify LPXNs influence on the CTNND1 promoter. In addition, it is not clear how and in response to which stimuli LPXN is transported into the nucleus. Investigation of these questions could further help to understand how LPXN acts on p120CTN expression.

The accumulation of mutations that leads to the deregulation of gene expression profiles is known as a hallmark of cancer. Subsequently, this deregulation ends in the disruption of signaling networks and molecular processes (Hanahan, Weinberg 2000; 2011). For LPXN the effects of its overexpression on PCa cells have been studied, but how deregulation of LPXN expression arises and how it is regulated in normal and PCa cells is unclear. Reporter gene assays of the promoter region of LPXN could be a first step to answer this question. These experiments will identify a region of interest and will lead to the identification of putative regulators that could easily be verified in human PCa cells. Possibly, these regulators might display putative targets for cancer therapy.

The generated LPXN knockout mouse model does not display any obvious abnormalities. These mice are viable and fertile and do not show any signs of discomfort. However, detailed studies on the LPXN knockout mouse are needed. These studies should include a confirmation of the absence of LPXN from the corresponding tissues. Proteins of the paxillin protein family were hypothesized to have distinct functions (Chen, Kroog 2010). However, these results were obtained from in vitro experiments and might not reflect the in vivo situation. Thus, if $\mathrm{LPXN}^{-} /$mice do not show a conspicuous phenotype they should be bred with ARA55 knockout mice to see if ARA55 may compensate for the loss 
of LPXN. In addition, analysis of LPXN expression concerning time and tissue-specificity could reveal, which tissues might be affected by a LPXN knockout and could display a starting point for further investigations. Since LPXN was originally found to be expressed in cells of hematopoietic origin, the analysis of these cells seems to be a promising option. Should following investigations require removement of LPXN from a specific cell or tissue type, the conditional LPXN knockout model maintains this possibility.

Taken together, LPXN was shown to be involved in a variety of signaling pathways and exert specific functions on the actin cytoskeleton to regulate PCa cell migration. In addition, LPXN turned out to be dispensable for proper development and represented potential to modulate radioresistance of PCa cells. 


\section{Summary}

Leupaxin is a focal adhesion protein of the paxillin protein family that is overexpressed in PCa. Recently, LPXN was shown to interact with the actin-binding protein $\mathrm{CaD}$ and to modulate its phosphorylation levels of CaD (Hardenberg 2010). In the present study the interaction of LPXN and CaD was confirmed. Moreover, LPXN was shown to interact with the phosphorylated form of $\mathrm{CaD}$ to modulate actin cytoskeletal dynamics during migration of PCa cells. To analyze the relevance of this interaction for cell migration, in situ proximity ligation assays (PLA) were performed on migrating PCa cells. The subcellular interaction pattern of LPXN and CaD showed increased interactions at the submembranous compartment, whereas interactions were absent from the leading edge of the migrating cell. This leads to the conclusion that $\mathrm{CaD}$ is phosphorylated at places where actin cytoskeletal structures need to be reassembled. A variety of kinases have been reported to phosphorylate $\mathrm{CaD}$, but only a few have already been described to regulate the actin affinity of $\mathrm{CaD}$ and would therefore display putative candidate kinases. A specific inhibitor for the mitogen-activated protein kinase kinase, MEK1, reduced the phosphorylation of $\mathrm{CaD}$ and identified the extracellular signal-regulated kinase 1/2 (ERK1/2) to be responsible for LPXN-dependent phosphorylation of CaD during migration of PCa cells. In addition, the interaction pattern of LPXN and activated ERK1/2 were similar to the one observed for LPXN-phospho-CaD interaction, leading to the hypothesis that ERK1/2 is recruited to phosphorylate CaD at sites of pre-dynamic actin cytoskeletal structures in a LPXN-dependent manner. Consistent with the increased LPXN expression in the highly invasive and androgen-independent PC-3 and DU145 cells, the proposed mechanism displays an explanation of how LPXN facilitates migration of PCa cells.

In the course of the present study we further analyzed the correlation of the expression of LPXN and the cell adhesion molecule $\beta 1$ integrin, which is known to be involved in the acquisition of radioresistance of several cancer entities (Ahmed et al. 2013). The resistance to therapeutic drugs and ionizing irradiation is a major obstacle in cancer therapy. In our PCa cells LPXN as well as $\beta 1$ integrin expression were inducible by ionizing irradiation. Knockdown of LPXN prevented this irradiation-induced upregulation of $\beta 1$ integrin and resulted in a radio-sensitization of the cells. Likewise, 
inhibition of $\beta 1$ integrin using an inhibitory antibody leads to a radio-sensitization of PCa cells. However, a simultaneous inhibition of $\beta 1$ integrin and downregulation of LPXN did not result in a synergistic effect, suggesting that LPXN and $\beta 1$ integrin might mediate resistance to ionizing irradiation through the same signaling pathway. To find a possible explanation for the LPXN-dependent expression of $\beta 1$ integrin the activity of the specificity protein 1 (SP1), which was shown to drive the expression of several integrins, was examined after knockdown and overexpression of LPXN. This assay revealed that only overexpression, rather than knockdown of LPXN was activating Sp1. This suggests that LPXN might mediate the expression of $\beta 1$ integrin through SP1. This hypothesis is supported by the fact that LPXN knockdown was affecting the activity of ERK1/2, which is regulating the DNA-binding activity of SP1.

Another part of the present study focused on the elucidation of the mechanism behind the negative correlation of LPXN and the cell adhesion molecule p120CTN. Because of its nuclear localization and coactivation of the androgen receptor, LPXN was suggested to have transcriptional activity. To identify LPXN-responsive elements, the p120CTN promoter was fragmented. Subsequent luciferase reporter assays identified a LPXNresponsive region ranging from $200 \mathrm{bp}$ upstream to $100 \mathrm{bp}$ downstream relative to the transcriptional start site. Further investigations using EMSA experiments did not uncover a LPXN binding site within this region of the p120CTN promoter. This suggests that LPXN might regulate p120CTN promoter activity indirectly through association with other proteins.

To investigate the physiologic role of LPXN a conditional knockout mouse was generated. Therefore, mouse embryonic stem (mES) cells with a targeted trap of the LPXN gene were purchased from the European conditional mouse mutagenesis program (EuCOMM). After excision of the resistance/reporter cassette these cells were injected into blastocysts but the resulting chimera did not show germ line transmission. Therefore, mES cells were subjected to cytogenetic analysis, which did not reveal numerical chromosome aberrations. After culturing in RESGRO ${ }^{\circledR}$ medium to remove ES cells that already started to differentiate before, mES cells were injected into blastocysts again. The resulting chimeras were bred with wild type C57BL/6 to establish a conditional LPXN knockout line. To determine the function and developmental importance of LPXN, it was deleted very early during development. To this end, LPXN flox/flox mice were bred with EIIA-Cre mice, which express the cre-recombinase in the early mouse embryo. 
LPXN-/- mice did not show any obvious phenotypic abnormalities and were viable and fertile, meaning that LPXN is not important for normal development.

In conclusion, the present study extended the understanding of LPXNs function in the progression of PCa. Although it was shown to be dispensable for embryonic development, LPXN clearly had a function in the migration of PCa cells. Furthermore, it possesses major influence on the radioresistance of PCa cells. Detailed analysis of the LPXN knockout mouse will provide a broader view of the processes affect by LPXN and will possibly contribute to improve or find new treatment options for patients suffering from PCa. 


\section{Bibliography}

Adam, L. P.; Gapinski, C. J.; Hathaway, D. R. (1992): Phosphorylation sequences in hcaldesmon from phorbol ester-stimulated canine aortas. In FEBS Lett 302 (3), pp. 223226.

Allende, L. M.; Hernandez, M.; Corell, A.; Garcia-Perez, M. A.; Varela, P.; Moreno, A. et al. (2000): A novel CD18 genomic deletion in a patient with severe leucocyte adhesion deficiency: a possible CD2/lymphocyte function-associated antigen-1 functional association in humans. In Immunology 99 (3), pp. 440-450.

Behrens, J. (1999): Cadherins and catenins: role in signal transduction and tumor progression. In Cancer Metastasis Rev 18 (1), pp. 15-30.

Beinke, C.; van Beuningen, D.; Cordes, N. (2003): Ionizing radiation modules of the expression and tyrosine phosphorylation of the focal adhesion-associated proteins focal adhesion kinase (FAK) and its substrates p130cas and paxillin in A549 human lung carcinoma cells in vitro. In International journal of radiation biology 79 (9), pp. 721-731.

Bellis, S. L.; Perrotta, J. A.; Curtis, M. S.; Turner, C. E. (1997): Adhesion of fibroblasts to fibronectin stimulates both serine and tyrosine phosphorylation of paxillin. In Biochem J 325 (Pt 2), pp. 375-381.

Birchmeier, W.; Behrens, J. (1994): Cadherin expression in carcinomas: role in the formation of cell junctions and the prevention of invasiveness. In Biochim Biophys Acta 1198 (1), pp. 11-26.

Birge, R. B.; Fajardo, J. E.; Reichman, C.; Shoelson, S. E.; Songyang, Z.; Cantley, L. C.; Hanafusa, H. (1993): Identification and characterization of a high-affinity interaction between v-Crk and tyrosine-phosphorylated paxillin in CT10-transformed fibroblasts. In Mol Cell Biol 13 (8), pp. 4648-4656. 
Border, W. A.; Noble, N. A. (1993): Cytokines in kidney disease: the role of transforming growth factor-beta. In Am J Kidney Dis 22 (1), pp. 105-113.

Bradford, M. M. (1976): A rapid and sensitive method for the quantitation of microgram quantities of protein utilizing the principle of protein-dye binding. In Anal Biochem 72, pp. 248-254.

Brown, M. C.; Perrotta, J. A.; Turner, C. E. (1996): Identification of LIM3 as the principal determinant of paxillin focal adhesion localization and characterization of a novel motif on paxillin directing vinculin and focal adhesion kinase binding. In J Cell Biol 135 (4), pp. 1109-1123.

Brown, M. C.; Perrotta, J. A.; Turner, C. E. (1998): Serine and threonine phosphorylation of the paxillin LIM domains regulates paxillin focal adhesion localization and cell adhesion to fibronectin. In Mol Biol Cell 9 (7), pp. 1803-1816.

Burridge, K.; Turner, C. E.; Romer, L. H. (1992): Tyrosine phosphorylation of paxillin and pp125FAK accompanies cell adhesion to extracellular matrix: a role in cytoskeletal assembly. In J Cell Biol 119 (4), pp. 893-903.

Chen, Pei-Wen; Kroog, Glenn S. (2010): Leupaxin is similar to paxillin in focal adhesion targeting and tyrosine phosphorylation but has distinct roles in cell adhesion and spreading. In Cell Adhesion \& Migration 4 (4), pp. 527-540.

Chetty, Runjan; Jain, Dhanpat; Serra, Stefano (2008): p120 catenin reduction and cytoplasmic relocalization leads to dysregulation of E-cadherin in solid pseudopapillary tumors of the pancreas. In Am J Clin Pathol 130 (1), pp. 71-76.

Chew, Valerie; Lam, Kong-Peng (2007): Leupaxin negatively regulates B cell receptor signaling. In J Biol Chem 282 (37), pp. 27181-27191.

Clark, J. M. (1988): Novel non-templated nucleotide addition reactions catalyzed by procaryotic and eucaryotic DNA polymerases. In Nucleic Acids Res 16 (20), pp. 96779686. 
Cohen, M. B.; Griebling, T. L.; Ahaghotu, C. A.; Rokhlin, O. W.; Ross, J. S. (1997): Cellular adhesion molecules in urologic malignancies. In Am J Clin Pathol 107 (1), pp. 56-63.

Cordes, N.; Blaese, M. A.; Meineke, V.; van Beuningen, D. (2002): Ionizing radiation induces up-regulation of functional beta1-integrin in human lung tumour cell lines in vitro. In Int J Radiat Biol 78 (5), pp. 347-357.

Cordes, Nils; Meineke, Viktor (2003): Cell adhesion-mediated radioresistance (CAMRR). Extracellular matrix-dependent improvement of cell survival in human tumor and normal cells in vitro. In Strahlenther Onkol 179 (5), pp. 337-344.

Cordes, Nils; Park, Catherine C. (2007): beta1 integrin as a molecular therapeutic target. In Int J Radiat Biol 83 (11-12), pp. 753-760.

Cordes, Nils; van Beuningen, Dirk (2004): Arrest of human lung fibroblasts in G2 phase after irradiation is regulated by converging phosphatidylinositol-3 kinase and beta1integrin signaling in vitro. In Int J Radiat Oncol Biol Phys 58 (2), pp. 453-462.

Cox, Elisabeth A.; Sastry, Sarita K.; Huttenlocher, Anna (2001): Integrin-mediated Adhesion Regulates Cell Polarity and Membrane Protrusion through the Rho Family of GTPases. In Mol Biol Cell 12 (2), pp. 265-277.

Cuomo, Maria Emanuela; Knebel, Axel; Platt, Georgina; Morrice, Nick; Cohen, Philip; Mittnacht, Sibylle (2005): Regulation of microfilament organization by Kaposi sarcomaassociated herpes virus-cyclin.CDK6 phosphorylation of caldesmon. In J Biol Chem 280 (43), pp. 35844-35858.

Dai, Hai-Ping; Xue, Yong-Quan; Zhou, Jian-Wei; Li, Ai-Ping; Wu, Ya-Fang; Pan, Jin-Lan et al. (2009): LPXN, a member of the paxillin superfamily, is fused to RUNX1 in an acute myeloid leukemia patient with a $\mathrm{t}(11 ; 21)(\mathrm{q} 12 ; \mathrm{q} 22)$ translocation. In Genes Chromosomes Cancer 48 (12), pp. 1027-1036. 
Damiano, J. S.; Cress, A. E.; Hazlehurst, L. A.; Shtil, A. A.; Dalton, W. S. (1999): Cell adhesion mediated drug resistance (CAM-DR): role of integrins and resistance to apoptosis in human myeloma cell lines. In Blood 93 (5), pp. 1658-1667.

D'Angelo, G.; Graceffa, P.; Wang, C. A.; Wrangle, J.; Adam, L. P. (1999): Mammal-specific, ERK-dependent, caldesmon phosphorylation in smooth muscle. Quantitation using novel anti-phosphopeptide antibodies. In The Journal of biological chemistry 274 (42), pp. 30115-30121.

Davis, Michael A.; Ireton, Renee C.; Reynolds, Albert B. (2003): A core function for p120catenin in cadherin turnover. In J Cell Biol 163 (3), pp. 525-534.

Dawid, I. B.; Breen, J. J.; Toyama, R. (1998): LIM domains: multiple roles as adapters and functional modifiers in protein interactions. In Trends Genet 14 (4), pp. 156-162.

Deng, Maoxian; Mohanan, Sunish; Polyak, Erzsebet; Chacko, Samuel (2007): Caldesmon is necessary for maintaining the actin and intermediate filaments in cultured bladder smooth muscle cells. In Cell Motil Cytoskeleton 64 (12), pp. 951-965.

Dierks, Sascha (2011): Analysis on the mechanisms underlying the leupaxin-mediated progression of prostate cancer. M.Sc. Georg August Universität Göttingen, Göttingen, Germany. Institut für Humangenetik.

Dierks, Sascha; Hardenberg, Sandra von; Schmidt, Thomas; Bremmer, Felix; Burfeind, Peter; Kaulfuß, Silke (2015): Leupaxin stimulates adhesion and migration of prostate cancer cells through modulation oft he phosphorylation status of the actin-binding protein caldesmon. Oncotarget 6 (15), pp. 13591 - 13606.

Dong, Jing-Ming; Lau, Lei-Shong; Ng, Yuen-Wai; Lim, Louis; Manser, Ed (2009): Paxillin nuclear-cytoplasmic localization is regulated by phosphorylation of the LD4 motif: evidence that nuclear paxillin promotes cell proliferation. In Biochem J 418 (1), pp. 173184. 
Edwards, Joanne; Bartlett, John M S (2005): The androgen receptor and signaltransduction pathways in hormone-refractory prostate cancer. Part 1: Modifications to the androgen receptor. In BJU Int 95 (9), pp. 1320-1326.

Eke, Iris; Deuse, Yvonne; Hehlgans, Stephanie; Gurtner, Kristin; Krause, Mechthild; Baumann, Michael et al. (2012): $\beta_{1}$ Integrin/FAK/cortactin signaling is essential for human head and neck cancer resistance to radiotherapy. In J. Clin. Invest. 122 (4), pp. $1529-1540$.

Ellerbroek, S. M.; Stack, M. S. (1999): Membrane associated matrix metalloproteinases in metastasis. In Bioessays 21 (11), pp. 940-949.

Eves, Robert; Webb, Bradley A.; Zhou, Shutang; Mak, Alan S. (2006): Caldesmon is an integral component of podosomes in smooth muscle cells. In J Cell Sci 119 (Pt 9), pp. 1691-1702.

Foster, D. B.; Shen, L. H.; Kelly, J.; Thibault, P.; van Eyk, J. E.; Mak, A. S. (2000): Phosphorylation of caldesmon by p21-activated kinase. Implications for the $\mathrm{Ca}(2+)$ sensitivity of smooth muscle contraction. In The Journal of biological chemistry 275 (3), pp. 1959-1965.

Friedrich, Anja; Kullmann, Frank (2003): Familial adenomatous polyposis syndrome (FAP): pathogenesis and molecular mechanisms. In Med Klin (Munich) 98 (12), pp. $776-$ 782.

Fujimoto, N.; Yeh, S.; Kang, H. Y.; Inui, S.; Chang, H. C.; Mizokami, A.; Chang, C. (1999): Cloning and characterization of androgen receptor coactivator, ARA55, in human prostate. In J Biol Chem 274 (12), pp. 8316-8321.

Gaudreault, Manon; Vigneault, Francois; Leclerc, Steeve; Guerin, Sylvain L. (2007): Laminin reduces expression of the human alpha6 integrin subunit gene by altering the level of the transcription factors Sp1 and Sp3. In Invest Ophthalmol Vis Sci 48 (8), pp. 3490-3505. 
George, E. L.; Georges-Labouesse, E. N.; Patel-King, R. S.; Rayburn, H.; Hynes, R. O. (1993): Defects in mesoderm, neural tube and vascular development in mouse embryos lacking fibronectin. In Development 119 (4), pp. 1079-1091.

Gershoni, J. M.; Palade, G. E. (1982): Electrophoretic transfer of proteins from sodium dodecyl sulfate-polyacrylamide gels to a positively charged membrane filter. In Anal Biochem 124 (2), pp. 396-405.

Gershoni, J. M.; Palade, G. E. (1983): Protein blotting: principles and applications. In Anal Biochem 131 (1), pp. 1-15.

Ghevaert, Cedric; Salsmann, Alexandre; Watkins, Nicholas A.; Schaffner-Reckinger, Elisabeth; Rankin, Angela; Garner, Stephen F. et al. (2008): A nonsynonymous SNP in the ITGB3 gene disrupts the conserved membrane-proximal cytoplasmic salt bridge in the alphallbbeta3 integrin and cosegregates dominantly with abnormal proplatelet formation and macrothrombocytopenia. In Blood 111 (7), pp. 3407-3414.

Giancotti, F. G.; Ruoslahti, E. (1999): Integrin signaling. In Science 285 (5430), pp. $1028-1032$.

Gingras, Marie-Eve; Larouche, Kathy; Larouche, Nicolas; Leclerc, Steeve; Salesse, Christian; Guerin, Sylvain L. (2003): Regulation of the integrin subunit alpha5 gene promoter by the transcription factors Sp1/Sp3 is influenced by the cell density in rabbit corneal epithelial cells. In Invest Ophthalmol Vis Sci 44 (9), pp. 3742-3755.

Goncharova, Elena A.; Vorotnikov, Alexander V.; Gracheva, Elena O.; Wang, C L Albert; Panettieri, Reynold A Jr; Stepanova, Victoria V.; Tkachuk, Vsevolod A. (2002): Activation of p38 MAP-kinase and caldesmon phosphorylation are essential for urokinase-induced human smooth muscle cell migration. In Biol Chem 383 (1), pp. 115-126.

Graham, Kathryn; Moran-Jones, Kim; Sansom, Owen J.; Brunton, Valerie G.; Frame, Margaret C. (2011): FAK deletion promotes p53-mediated induction of p21, DNAdamage responses and radio-resistance in advanced squamous cancer cells. In PLoS One 6 (12), pp. e27806. 
Grimsley, Cynthia M.; Kinchen, Jason M.; Tosello-Trampont, Annie-Carole; Brugnera, Enrico; Haney, Lisa B.; Lu, Mingjian et al. (2004): Dock180 and ELMO1 proteins cooperate to promote evolutionarily conserved Rac-dependent cell migration. In J Biol Chem 279 (7), pp. 6087-6097.

Gruber, G.; Hess, J.; Stiefel, C.; Aebersold, D. M.; Zimmer, Y.; Greiner, R. H. et al. (2005): Correlation between the tumoral expression of beta3-integrin and outcome in cervical cancer patients who had undergone radiotherapy. In Br J Cancer 92 (1), pp. 41-46.

Gu, Zhizhan; Kordowska, Jolanta; Williams, Geoffrey L.; Wang, C-L Albert; Hai, Chi-Ming (2007): Erk1/2 MAPK and caldesmon differentially regulate podosome dynamics in A7r5 vascular smooth muscle cells. In Exp Cell Res 313 (5), pp. 849-866.

Gupta, Anandarup; Lee, Beth S.; Khadeer, Mohammed A.; Tang, Zhihui; Chellaiah, Meenakshi; Abu-Amer, Yousef et al. (2003): Leupaxin is a critical adaptor protein in the adhesion zone of the osteoclast. In Journal of bone and mineral research : the official journal of the American Society for Bone and Mineral Research 18 (4), pp. 669-685.

Hagel, Margit; George, Elizabeth L.; Kim, Ann; Tamimi, Rulla; Opitz, Sarah L.; Turner, Christopher E. et al. (2002): The adaptor protein paxillin is essential for normal development in the mouse and is a critical transducer of fibronectin signaling. In Molecular and cellular biology 22 (3), pp. 901-915.

Halder, Jyotsnabaran; Landen, Charles N Jr; Lutgendorf, Susan K.; Li, Yang; Jennings, Nicholas B.; Fan, Dominic et al. (2005): Focal adhesion kinase silencing augments docetaxel-mediated apoptosis in ovarian cancer cells. In Clin Cancer Res 11 (24 Pt 1), pp. 8829-8836.

Hallahan, D. E.; Virudachalam, S. (1997): Ionizing radiation mediates expression of cell adhesion molecules in distinct histological patterns within the lung. In Cancer research 57 (11), pp. 2096-2099. 
Hanahan, D. (1983): Studies on transformation of Escherichia coli with plasmids. In $J$ Mol Biol 166 (4), pp. 557-580.

Hanahan, D.; Weinberg, R. A. (2000): The hallmarks of cancer. In Cell 100 (1), pp. 57-70.

Hanahan, D.; Weinbger, R. A. (2011): Hallmarks of cancer: the next generation. In Cell 144 (5), pp. 646-74.

Hardenberg, Sandra von (2010): Charakterisierung von Leupaxin und seiner Interaktionspartner in Karzinomzellen. Georg August Universität Göttingen, Göttingen, Germany. Institut für Humangenetik.

Haruna, M.; Hayashi, K.; Yano, H.; Takeuchi, O.; Sobue, K. (1993): Common structural and expressional properties of vertebrate caldesmon genes. In Biochem Biophys Res Commun 197 (1), pp. 145-153.

Hayashi, K.; Yano, H.; Hashida, T.; Takeuchi, R.; Takeda, O.; Asada, K. et al. (1992): Genomic structure of the human caldesmon gene. In Proc Natl Acad Sci U S A 89 (24), pp. $12122-12126$.

Hazlehurst, L. A.; Damiano, J. S.; Buyuksal, I.; Pledger, W. J.; Dalton, W. S. (2000): Adhesion to fibronectin via beta1 integrins regulates p27kip1 levels and contributes to cell adhesion mediated drug resistance (CAM-DR). In Oncogene 19 (38), pp. 4319-4327.

Heitzer, Marjet D.; Defranco, Donald B. (2006): Hic-5, an adaptor-like nuclear receptor coactivator. In Nucl Recept Signal 4.

Herzig, M.; Savarese, F.; Novatchkova, M.; Semb, H.; Christofori, G. (2007): Tumor progression induced by the loss of E-cadherin independent of beta-catenin/Tcfmediated Wnt signaling. In Oncogene 26 (16), pp. 2290-2298.

Hodkinson, P. S.; Elliott, T.; Wong, W. S.; Rintoul, R. C.; MacKinnon, A. C.; Haslett, C.; Sethi, T. (2006): ECM overrides DNA damage-induced cell cycle arrest and apoptosis in small- 
cell lung cancer cells through beta1 integrin-dependent activation of PI3-kinase. In Cell Death Differ 13 (10), pp. 1776-1788.

Horoszewicz, J. S.; Leong, S. S.; Kawinski, E.; Karr, J. P.; Rosenthal, H.; Chu, T. M. et al. (1983): LNCaP model of human prostatic carcinoma. In Cancer Res 43 (4), pp. 18091818.

Hu, G. (1993): DNA polymerase-catalyzed addition of nontemplated extra nucleotides to the 3' end of a DNA fragment. In DNA Cell Biol 12 (8), pp. 763-770.

Hynes, Richard O. (2002a): Integrins: bidirectional, allosteric signaling machines. In Cell 110 (6), pp. 673-687.

Hynes, Richard O. (2002b): Integrins: bidirectional, allosteric signaling machines. In Cell 110 (6), pp. 673-687.

Ishikawa, R.; Kagami, O.; Hayashi, C.; Kohama, K. (1992a): Characterization of smooth muscle caldesmon as a microtubule-associated protein. In Cell Motil Cytoskeleton 23 (4), pp. 244-251.

Ishikawa, R.; Kagami, O.; Hayashi, C.; Kohama, K. (1992b): The binding of nonmuscle caldesmon from brain to microtubules. Regulations by $\mathrm{Ca}(2+)$-calmodulin and cdc2 kinase. In FEBS Lett 299 (1), pp. 54-56.

Ishikawa, R.; Yamashiro, S.; Kohama, K.; Matsumura, F. (1998): Regulation of actin binding and actin bundling activities of fascin by caldesmon coupled with tropomyosin. In The Journal of biological chemistry 273 (41), pp. 26991-26997.

Jaffe, Aron B.; Hall, Alan (2005): Rho GTPases: biochemistry and biology. In Annu Rev Cell Dev Biol 21, pp. 247-269.

Jeanes, A.; Gottardi, C. J.; Yap, A. S. (2008): Cadherins and cancer: how does cadherin dysfunction promote tumor progression? In Oncogene 27 (55), pp. 6920-6929. 
Jeon, Kwang W. (2009): International review of cell and molecular biology. London: Academic.

Kadonaga, J. T.; Courey, A. J.; Ladika, J.; Tjian, R. (1988): Distinct regions of Sp1 modulate DNA binding and transcriptional activation. In Science 242 (4885), pp. 15661570.

Kadrmas, Julie L.; Beckerle, Mary C. (2004): The LIM domain: from the cytoskeleton to the nucleus. In Nat Rev Mol Cell Biol 5 (11), pp. 920-931.

Kaighn, M. E.; Narayan, K. S.; Ohnuki, Y.; Lechner, J. F.; Jones, L. W. (1979): Establishment and characterization of a human prostatic carcinoma cell line (PC-3). In Invest Urol 17 (1), pp. 16-23.

Kallakury, B. V.; Sheehan, C. E.; Winn-Deen, E.; Oliver, J.; Fisher, H. A.; Kaufman, R P Jr; Ross, J. S. (2001): Decreased expression of catenins (alpha and beta), p120 CTN, and Ecadherin cell adhesion proteins and E-cadherin gene promoter methylation in prostatic adenocarcinomas. In Cancer 92 (11), pp. 2786-2795.

Kaulfuss, S.; Hardenberg, S. von; Schweyer, S.; Herr, A. M.; Laccone, F.; Wolf, S.; Burfeind, P. (2009): Leupaxin acts as a mediator in prostate carcinoma progression through deregulation of p120catenin expression. In Oncogene 28 (45), pp. 3971-3982.

Kaulfuss, Silke (2006): Zur Funktion von Leupaxin beim Karzinom der Prostata. Georg August Universität Göttingen, Göttingen, Germany. Institut für Humangenetik

Kaulfuss, Silke; Grzmil, Michal; Hemmerlein, Bernhard; Thelen, Paul; Schweyer, Stefan; Neesen, Jürgen et al. (2008): Leupaxin, a novel coactivator of the androgen receptor, is expressed in prostate cancer and plays a role in adhesion and invasion of prostate carcinoma cells. In Molecular endocrinology (Baltimore, Md.) 22 (7), pp. 1606-1621.

Kilpelainen, Tuomas P.; Tammela, Teuvo L J; Roobol, Monique; Hugosson, Jonas; Ciatto, Stefano; Nelen, Vera et al. (2011): False-positive screening results in the European 
randomized study of screening for prostate cancer. In Eur J Cancer 47 (18), pp. 26982705.

Kim, Kyung-Hee; Yeo, Seung-Gu; Kim, Won Ki; Kim, Dae Yong; Yeo, Hyun Yang; Hong, Jun Pyu et al. (2012): Up-regulated expression of l-caldesmon associated with malignancy of colorectal cancer. In BMC Cancer 12 (1), p. 601. DOI: 10.1186/1471-240712-601.

Kim-Kaneyama, Joo-ri; Takeda, Naoki; Sasai, Asami; Miyazaki, Akira; Sata, Masataka; Hirabayashi, Takahiro et al. (2011): Hic-5 deficiency enhances mechanosensitive apoptosis and modulates vascular remodeling. In J Mol Cell Cardiol 50 (1), pp. 77-86.

Kiziltepe, T.; Ashley, J. D.; Stefanick, J. F.; Qi, Y. M.; Alves, N. J.; Handlogten, M. W. et al. (2012): Rationally engineered nanoparticles target multiple myeloma cells, overcome cell-adhesion-mediated drug resistance, and show enhanced efficacy in vivo. In Blood Cancer J 2 (4), pp. e64-.

Kordowska, Jolanta; Huang, Renjian; Wang, Chih-Lueh Albert (2006): Phosphorylation of caldesmon during smooth muscle contraction and cell migration or proliferation. In $J$ Biomed Sci 13 (2), pp. 159-172.

Kraus, Alison C.; Ferber, Ines; Bachmann, Sven-Oliver; Specht, Hannah; Wimmel, Anja; Gross, Markus W. et al. (2002): In vitro chemo- and radio-resistance in small cell lung cancer correlates with cell adhesion and constitutive activation of AKT and MAP kinase pathways. In Oncogene 21 (57), pp. 8683-8695.

Laemmli, U. K. (1970): Cleavage of structural proteins during the assembly of the head of bacteriophage T4. In Nature 227 (5259), pp. 680-685.

Lau, Tong-Lay; Kim, Chungho; Ginsberg, Mark H.; Ulmer, Tobias S. (2009): The structure of the integrin alphallbbeta3 transmembrane complex explains integrin transmembrane signalling. In EMBO J 28 (9), pp. 1351-1361. 
Li, Y.; Wessels, D.; Wang, T.; Lin, J L C; Soll, D. R.; Lin, J J C (2003): Regulation of caldesmon activity by Cdc2 kinase plays an important role in maintaining membrane cortex integrity during cell division. In Cell Mol Life Sci 60 (1), pp. 198-211.

Lin, Jim Jung-Ching; Li, Yan; Eppinga, Robbin D.; Wang, Qinchuan; Jin, Jian-Ping (2009): Chapter 1: roles of caldesmon in cell motility and actin cytoskeleton remodeling. In International review of cell and molecular biology 274, pp. 1-68.

Lipsky, B. P.; Beals, C. R.; Staunton, D. E. (1998): Leupaxin is a novel LIM domain protein that forms a complex with PYK2. In The Journal of biological chemistry 273 (19), pp. 11709-11713.

Liu, Yang; Li, Qing-Chang; Miao, Yuan; Xu, Hong-Tao; Dai, Shun-Dong; Wei, Qiang et al. (2009): Ablation of p120-catenin enhances invasion and metastasis of human lung cancer cells. In Cancer Sci 100 (3), pp. 441-448.

Lois, C.; Refaeli, Y.; Qin, X. F.; van Parijs, L. (2001): Retroviruses as tools to study the immune system. In Curr Opin Immunol 13 (4), pp. 496-504.

Mak, A. S.; Watson, M. H.; Litwin, C. M.; Wang, J. H. (1991): Phosphorylation of caldesmon by cdc2 kinase. In J Biol Chem 266 (11), pp. 6678-6681.

Maniatis, Tom; Fritsch, E. F.; Sambrook, Joseph (1982): Molecular cloning. A laboratory manual. Cold Spring Harbor, N.Y.: Cold Spring Harbor Laboratory.

Matsumura, F.; Yamashiro, S. (1993): Caldesmon. In Curr Opin Cell Biol 5 (1), pp. 70-76.

Mege, Rene-Marc; Gavard, Julie; Lambert, Mireille (2006): Regulation of cell-cell junctions by the cytoskeleton. In Curr Opin Cell Biol 18 (5), pp. 541-548.

Merchant, J. L.; Du, M.; Todisco, A. (1999): Sp1 phosphorylation by Erk 2 stimulates DNA binding. In Biochem Biophys Res Commun 254 (2), pp. 454-461. 
Milanini-Mongiat, Julie; Pouyssegur, Jacques; Pages, Gilles (2002): Identification of two Sp1 phosphorylation sites for $\mathrm{p} 42 / \mathrm{p} 44$ mitogen-activated protein kinases: their implication in vascular endothelial growth factor gene transcription. In J Biol Chem 277 (23), pp. 20631-20639.

Mortazavi, Fariborz; An, Jiabin; Dubinett, Steven; Rettig, Matthew (2010): p120-catenin is transcriptionally downregulated by FOXC2 in non-small cell lung cancer cells. In $\mathrm{Mol}$ Cancer Res 8 (5), pp. 762-774.

Mortazavi, Fariborz; Dubinett, Steven; Rettig, Matthew (2011): c-Crk proto-oncogene contributes to transcriptional repression of p120-catenin in non-small cell lung cancer cells. In Clin Exp Metastasis 28 (4), pp. 391-404.

Muller, Judith M.; Metzger, Eric; Greschik, Holger; Bosserhoff, Anja-Katrin; Mercep, Luka; Buettner, Reinhard; Schule, Roland (2002): The transcriptional coactivator FHL2 transmits Rho signals from the cell membrane into the nucleus. In EMBO J 21 (4), pp. 736-748.

Ng, Lui; Tung-Ping Poon, Ronnie; Yau, Simon; Chow, Ariel; Lam, Colin; Li, Hung-Sing et al. (2013): Suppression of actopaxin impairs hepatocellular carcinoma metastasis through modulation of cell migration and invasion. In Hepatology 58 (2), pp. 667-679.

Ni, Jie; Cozzi, Paul; Hao, Jingli; Beretov, Julia; Chang, Lei; Duan, Wei et al. (2013): Epithelial cell adhesion molecule (EpCAM) is associated with prostate cancer metastasis and chemo/radioresistance via the PI3K/Akt/mTOR signaling pathway. In Int J Biochem Cell Biol 45 (12), pp. 2736-2748.

Nikolopoulos, S. N.; Turner, C. E. (2000): Actopaxin, a new focal adhesion protein that binds paxillin LD motifs and actin and regulates cell adhesion. In J Cell Biol 151 (7), pp. $1435-1448$.

Nishiya, N.; Sabe, H.; Nose, K.; Shibanuma, M. (1998): The LIM domains of hic-5 protein recognize specific DNA fragments in a zinc-dependent manner in vitro. In Nucleic Acids Res 26 (18), pp. 4267-4273. 
Nishiya, N.; Tachibana, K.; Shibanuma, M.; Mashimo, J. I.; Nose, K. (2001): Hic-5-reduced cell spreading on fibronectin: competitive effects between paxillin and Hic-5 through interaction with focal adhesion kinase. In Molecular and cellular biology 21 (16), pp. 5332-5345.

Nix, D. A.; Beckerle, M. C. (1997): Nuclear-cytoplasmic shuttling of the focal contact protein, zyxin: a potential mechanism for communication between sites of cell adhesion and the nucleus. In J Cell Biol 138 (5), pp. 1139-1147.

Nomura, M.; Yoshikawa, K.; Tanaka, T.; Sobue, K.; Maruyama, K. (1987): The role of tropomyosin in the interactions of F-actin with caldesmon and actin-binding protein (or filamin). In European journal of biochemistry / FEBS 163 (3), pp. 467-471.

Novy, R. E.; Lin, J. L.; Lin, J. J. (1991): Characterization of cDNA clones encoding a human fibroblast caldesmon isoform and analysis of caldesmon expression in normal and transformed cells. In J Biol Chem 266 (25), pp. 16917-16924.

Nübel, Tobias; Dippold, Wolfgang; Kaina, Bernd; Fritz, Gerhard (2004): Ionizing radiation-induced E-selectin gene expression and tumor cell adhesion is inhibited by lovastatin and all-trans retinoic acid. In Carcinogenesis 25 (8), pp. 1335-1344.

Ohnuki, Y.; Marnell, M. M.; Babcock, M. S.; Lechner, J. F.; Kaighn, M. E. (1980): Chromosomal analysis of human prostatic adenocarcinoma cell lines. In Cancer Res 40 (3), pp. 524-534.

Okegawa, Takatsugu; Li, Yingming; Pong, Rey-Chen; Hsieh, Jer-Tsong (2002): Cell adhesion proteins as tumor suppressors. In J Urol 167 (4), pp. 1836-1843.

Onder, Tamer T.; Gupta, Piyush B.; Mani, Sendurai A.; Yang, Jing; Lander, Eric S.; Weinberg, Robert A. (2008): Loss of E-cadherin promotes metastasis via multiple downstream transcriptional pathways. In Cancer Res 68 (10), pp. 3645-3654. 
Onishi, T.; Yamakawa, K.; Franco, O. E.; Kawamura, J.; Watanabe, M.; Shiraishi, T.; Kitazawa, S. (2001): Mitogen-activated protein kinase pathway is involved in alpha6 integrin gene expression in androgen-independent prostate cancer cells: role of proximal Sp1 consensus sequence. In Biochim Biophys Acta 1538 (2-3), pp. 218-227.

Owada, M. K.; Hakura, A.; Iida, K.; Yahara, I.; Sobue, K.; Kakiuchi, S. (1984): Occurrence of caldesmon (a calmodulin-binding protein) in cultured cells: comparison of normal and transformed cells. In Proc Natl Acad Sci U S A 81 (10), pp. 3133-3137.

Paoli, Paolo; Giannoni, Elisa; Chiarugi, Paola (2013): Anoikis molecular pathways and its role in cancer progression. In Biochim Biophys Acta 1833 (12), pp. 3481-3498.

Paredes, Joana; Figueiredo, Joana; Albergaria, André; Oliveira, Patrícia; Carvalho, Joana; Ribeiro, Ana Sofia et al. (2012): Epithelial E- and P-cadherins: role and clinical significance in cancer. In Biochimica et biophysica acta 1826 (2), pp. 297-311.

Perez-Moreno, Mirna; Song, Weimin; Pasolli, H. Amalia; Williams, Scott E.; Fuchs, Elaine (2008): Loss of p120 catenin and links to mitotic alterations, inflammation, and skin cancer. In Proc Natl Acad Sci U S A 105 (40), pp. 15399-15404.

Perl, A. K.; Wilgenbus, P.; Dahl, U.; Semb, H.; Christofori, G. (1998): A causal role for Ecadherin in the transition from adenoma to carcinoma. In Nature 392 (6672), pp. 190193.

Petit, V.; Boyer, B.; Lentz, D.; Turner, C. E.; Thiery, J. P.; Valles, A. M. (2000): Phosphorylation of tyrosine residues 31 and 118 on paxillin regulates cell migration through an association with CRK in NBT-II cells. In J Cell Biol 148 (5), pp. 957-970.

Pontes-Junior, Jose; Reis, Sabrina Thalita; de Oliveira, Luis Carlos Neves; Sant'anna, Alexandre C.; Dall'oglio, Marcos Francisco; Antunes, Alberto Azoubel et al. (2010): Association between integrin expression and prognosis in localized prostate cancer. In Prostate 70 (11), pp. 1189-1195. 
Reynolds, A. B.; Roesel, D. J.; Kanner, S. B.; Parsons, J. T. (1989): Transformation-specific tyrosine phosphorylation of a novel cellular protein in chicken cells expressing oncogenic variants of the avian cellular src gene. In Mol Cell Biol 9 (2), pp. 629-638.

Ridley, A. J. (2003): Cell Migration: Integrating Signals from Front to Back. In Science 302, pp. 1704-1709.

Roman, Horia Nicolae; Zitouni, Nedjma B.; Kachmar, Linda; Benedetti, Andrea; Sobieszek, Apolinary; Lauzon, Anne-Marie (2014): The role of caldesmon and its phosphorylation by ERK on the binding force of unphosphorylated myosin to actin. In Biochim Biophys Acta 1840 (11), pp. 3218-3225.

Romig, H.; Fackelmayer, F. O.; Renz, A.; Ramsperger, U.; Richter, A. (1992): Characterization of SAF-A, a novel nuclear DNA binding protein from HeLa cells with high affinity for nuclear matrix/scaffold attachment DNA elements. In EMBO J 11 (9), pp. 3431-3440.

Ross, D. T.; Scherf, U.; Eisen, M. B.; Perou, C. M.; Rees, C.; Spellman, P. et al. (2000): Systematic variation in gene expression patterns in human cancer cell lines. In Nat Genet 24 (3), pp. 227-235.

Ruzzi, L.; Gagnoux-Palacios, L.; Pinola, M.; Belli, S.; Meneguzzi, G.; D'Alessio, M.; Zambruno, G. (1997): A homozygous mutation in the integrin alpha6 gene in junctional epidermolysis bullosa with pyloric atresia. In J Clin Invest 99 (12), pp. 2826-2831.

Sahu, Surasri Nandan; Khadeer, Mohammed Abdul; Robertson, Brian W.; Núñez, Stephanie M.; Bai, Guang; Gupta, Anandarup (2007a): Association of leupaxin with Src in osteoclasts. In American journal of physiology. Cell physiology 292 (1), pp. C581-90.

Sahu, Surasri Nandan; Nunez, Stephanie; Bai, Guang; Gupta, Anandarup (2007b): Interaction of Pyk2 and PTP-PEST with leupaxin in prostate cancer cells. In Am J Physiol Cell Physiol 292 (6), pp. C2288-96. 
Saiki, R. K.; Scharf, S.; Faloona, F.; Mullis, K. B.; Horn, G. T.; Erlich, H. A.; Arnheim, N. (1992): Enzymatic amplification of beta-globin genomic sequences and restriction site analysis for diagnosis of sickle cell anemia. 1985. In Biotechnology 24, pp. 476-480.

Sandfort, V.; Koch, U.; Cordes, N. (2007): Cell adhesion-mediated radioresistance revisited. In Int J Radiat Biol 83 (11-12), pp. 727-732.

Sanger, F.; Coulson, A. R. (1975): A rapid method for determining sequences in DNA by primed synthesis with DNA polymerase. In J Mol Biol 94 (3), pp. 441-448.

Schackmann, Ron C J; Klarenbeek, Sjoerd; Vlug, Eva J.; Stelloo, Suzan; van Amersfoort, Miranda; Tenhagen, Milou et al. (2013): Loss of p120-catenin induces metastatic progression of breast cancer by inducing anoikis resistance and augmenting growth factor receptor signaling. In Cancer Res 73 (15), pp. 4937-4949.

Schaft, J.; Ashery-Padan, R.; van der Hoeven, F; Gruss, P.; Stewart, A. F. (2001): Efficient FLP recombination in mouse ES cells and oocytes. In Genesis 31 (1), pp. 6-10.

Schaller, M. D. (2001): Paxillin: a focal adhesion-associated adaptor protein. In Oncogene 20 (44), pp. 6459-6472.

Schaller, M. D.; Parsons, J. T. (1995): pp125FAK-dependent tyrosine phosphorylation of paxillin creates a high-affinity binding site for Crk. In Mol Cell Biol 15 (5), pp. 2635-2645.

Schneikert, Jean; Behrens, Jurgen (2007): The canonical Wnt signalling pathway and its APC partner in colon cancer development. In Gut 56 (3), pp. 417-425.

Seidler, Julia; Durzok, Rita; Brakebusch, Cord; Cordes, Nils (2005): Interactions of the integrin subunit beta1A with protein kinase B/Akt, p130Cas and paxillin contribute to regulation of radiation survival. In Radiother Oncol 76 (2), pp. 129-134.

Sen, Aritro; Castro, Ismary de; Defranco, Donald B.; Deng, Fang-Ming; Melamed, Jonathan; Kapur, Payel et al. (2012): Paxillin mediates extranuclear and intranuclear signaling in prostate cancer proliferation. In J Clin Invest 122 (7), pp. 2469-2481. 
Sethi, T.; Rintoul, R. C.; Moore, S. M.; MacKinnon, A. C.; Salter, D.; Choo, C. et al. (1999): Extracellular matrix proteins protect small cell lung cancer cells against apoptosis: a mechanism for small cell lung cancer growth and drug resistance in vivo. In Nat Med 5 (6), pp. 662-668.

Shattil, Sanford J.; Kim, Chungho; Ginsberg, Mark H. (2010): The final steps of integrin activation: the end game. In Nat Rev Mol Cell Biol 11 (4), pp. 288-300.

Shen, Y.; Schneider, G.; Cloutier, J. F.; Veillette, A.; Schaller, M. D. (1998): Direct association of protein-tyrosine phosphatase PTP-PEST with paxillin. In J Biol Chem 273 (11), pp. 6474-6481.

Shibanuma, Motoko; Kim-Kaneyama, Joo-ri; Ishino, Keiko; Sakamoto, Nobuko; Hishiki, Tomoko; Yamaguchi, Kaeko et al. (2003): Hic-5 communicates between focal adhesions and the nucleus through oxidant-sensitive nuclear export signal. In Mol Biol Cell 14 (3), pp. 1158-1171.

Sisci, Diego; Middea, Emilia; Morelli, Catia; Lanzino, Marilena; Aquila, Saveria; Rizza, Pietro et al. (2010): 17beta-estradiol enhances alpha(5) integrin subunit gene expression through ERalpha-Sp1 interaction and reduces cell motility and invasion of ERalpha-positive breast cancer cells. In Breast Cancer Res Treat 124 (1), pp. 63-77.

Stairs, Douglas B.; Bayne, Lauren J.; Rhoades, Ben; Vega, Maria E.; Waldron, Todd J.; Kalabis, Jiri et al. (2011): Deletion of p120-catenin results in a tumor microenvironment with inflammation and cancer that establishes it as a tumor suppressor gene. In Cancer Cell 19 (4), pp. 470-483.

Stetler-Stevenson, W. G. (2001): The role of matrix metalloproteinases in tumor invasion, metastasis, and angiogenesis. In Surg Oncol Clin N Am 10 (2), pp. 383-92, x.

Stone, K. R.; Mickey, D. D.; Wunderli, H.; Mickey, G. H.; Paulson, D. F. (1978): Isolation of a human prostate carcinoma cell line (DU 145). In Int J Cancer 21 (3), pp. 274-281. 
Subauste, M. Cecilia; Pertz, Olivier; Adamson, Eileen D.; Turner, Christopher E.; Junger, Sachiko; Hahn, Klaus M. (2004): Vinculin modulation of paxillin-FAK interactions regulates ERK to control survival and motility. In J Cell Biol 165 (3), pp. 371-381.

Sugawara, Ayako; Goto, Kazuo; Sotomaru, Yusuke; Sofuni, Toshio; Ito, Toshio (2006): Current status of chromosomal abnormalities in mouse embryonic stem cell lines used in Japan. In Comp Med 56 (1), pp. 31-34.

Sundberg-Smith, Liisa J.; DiMichele, Laura A.; Sayers, Rebecca L.; Mack, Christopher P.; Taylor, Joan M. (2008): The LIM protein leupaxin is enriched in smooth muscle and functions as an serum response factor cofactor to induce smooth muscle cell gene transcription. In Circulation research 102 (12), pp. 1502-1511.

Tanaka, J.; Watanabe, T.; Nakamura, N.; Sobue, K. (1993): Morphological and biochemical analyses of contractile proteins (actin, myosin, caldesmon and tropomyosin) in normal and transformed cells. In J Cell Sci 104 (Pt 2), pp. 595-606.

Thiery, Jean Paul (2002): Epithelial-mesenchymal transitions in tumour progression. In Nat Rev Cancer 2 (6), pp. 442-454.

Thomas, S. M.; Hagel, M.; Turner, C. E. (1999): Characterization of a focal adhesion protein, Hic-5, that shares extensive homology with paxillin. In J Cell Sci 112 (Pt 2), pp. 181-190.

Tong, X.; Salgia, R.; Li, J. L.; Griffin, J. D.; Howley, P. M. (1997): The bovine papillomavirus E6 protein binds to the LD motif repeats of paxillin and blocks its interaction with vinculin and the focal adhesion kinase. In J Biol Chem 272 (52), pp. 33373-33376.

Trachtenberg, John; Blackledge, George (2002): Looking to the Future: Advances in the Management of Hormone-Refractory Prostate Cancer. In European Urology Supplements 1 (7), pp. 44-53.

Tsubouchi, Asako; Sakakura, Junko; Yagi, Ryohei; Mazaki, Yuichi; Schaefer, Erik; Yano, Hajime; Sabe, Hisataka (2002): Localized suppression of RhoA activity by Tyr31/118- 
phosphorylated paxillin in cell adhesion and migration. In J Cell Biol 159 (4), pp. 673683.

Turner, C. E. (2000): Paxillin interactions. In Journal of cell science 113 Pt 23, pp. 41394140.

Turner, C. E.; Brown, M. C.; Perrotta, J. A.; Riedy, M. C.; Nikolopoulos, S. N.; McDonald, A. R. et al. (1999): Paxillin LD4 motif binds PAK and PIX through a novel 95-kD ankyrin repeat, ARF-GAP protein: A role in cytoskeletal remodeling. In J Cell Biol 145 (4), pp. 851-863.

Turner, C. E.; Glenney, J R Jr; Burridge, K. (1990): Paxillin: a new vinculin-binding protein present in focal adhesions. In J Cell Biol 111 (3), pp. 1059-1068.

Valles, Ana M.; Beuvin, Maud; Boyer, Brigitte (2004): Activation of Rac1 by paxillin-CrkDOCK180 signaling complex is antagonized by Rap1 in migrating NBT-II cells. In J Biol Chem 279 (43), pp. 44490-44496.

Vande Pol, S B; Brown, M. C.; Turner, C. E. (1998): Association of Bovine Papillomavirus Type 1 E6 oncoprotein with the focal adhesion protein paxillin through a conserved protein interaction motif. In Oncogene 16 (1), pp. 43-52.

von Kries, J P; Buhrmester, H.; Stratling, W. H. (1991): A matrix/scaffold attachment region binding protein: identification, purification, and mode of binding. In Cell 64 (1), pp. 123-135.

Wade, Ramon; Bohl, Joanna; Vande Pol, Scott (2002): Paxillin null embryonic stem cells are impaired in cell spreading and tyrosine phosphorylation of focal adhesion kinase. In Oncogene 21 (1), pp. 96-107.

Wang, Yuan; Gilmore, Thomas D. (2003): Zyxin and paxillin proteins: focal adhesion plaque LIM domain proteins go nuclear. In Biochim Biophys Acta 1593 (2-3), pp. 115120. 
Watanabe, K.; Tajino, T.; Sekiguchi, M.; Suzuki, T. (2000): h-Caldesmon as a specific marker for smooth muscle tumors. Comparison with other smooth muscle markers in bone tumors. In Am J Clin Pathol 113 (5), pp. 663-668.

Watt, F. M.; Hodivala, K. J. (1994): Cell adhesion. Fibronectin and integrin knockouts come unstuck. In Curr Biol 4 (3), pp. 270-272.

Way, J. C.; Chalfie, M. (1988): mec-3, a homeobox-containing gene that specifies differentiation of the touch receptor neurons in C. elegans. In Cell 54 (1), pp. 5-16.

Wijnhoven, B. P.; Dinjens, W. N.; Pignatelli, M. (2000): E-cadherin-catenin cell-cell adhesion complex and human cancer. In Br J Surg 87 (8), pp. 992-1005.

Woods, Alison J.; Roberts, Marnie S.; Choudhary, Jyoti; Barry, Simon T.; Mazaki, Yuichi; Sabe, Hisataka et al. (2002): Paxillin associates with poly(A)-binding protein 1 at the dense endoplasmic reticulum and the leading edge of migrating cells. In J Biol Chem 277 (8), pp. 6428-6437.

Wu, D-W; Wu, T-C; Wu, J-Y; Cheng, Y-W; Chen, Y-C; Lee, M-C et al. (2014): Phosphorylation of paxillin confers cisplatin resistance in non-small cell lung cancer via activating ERK-mediated Bcl-2 expression. In Oncogene 33 (35), pp. 4385-4395.

Yamakita, Yoshihiko; Oosawa, Fumio; Yamashiro, Shigeko; Matsumura, Fumio (2003): Caldesmon inhibits Arp2/3-mediated actin nucleation. In The Journal of biological chemistry 278 (20), pp. 17937-17944.

Yamboliev, I. A.; Gerthoffer, W. T. (2001): Modulatory role of ERK MAPK-caldesmon pathway in PDGF-stimulated migration of cultured pulmonary artery SMCs. In American journal of physiology. Cell physiology 280 (6), pp. C1680-8.

Yang, L.; Guerrero, J.; Hong, H.; DeFranco, D. B.; Stallcup, M. R. (2000): Interaction of the tau2 transcriptional activation domain of glucocorticoid receptor with a novel steroid receptor coactivator, Hic-5, which localizes to both focal adhesions and the nuclear matrix. In Mol Biol Cell 11 (6), pp. 2007-2018. 
Yap, A. S. (1998): The morphogenetic role of cadherin cell adhesion molecules in human cancer: a thematic review. In Cancer Invest 16 (4), pp. 252-261.

Yokouchi, Kazuhiko; Numaguchi, Yasushi; Kubota, Ryuji; Ishii, Masakazu; Imai, Hajime; Murakami, Ryuichiro et al. (2006): l-Caldesmon regulates proliferation and migration of vascular smooth muscle cells and inhibits neointimal formation after angioplasty. In Arterioscler Thromb Vasc Biol 26 (10), pp. 2231-2237.

Yoshio, T.; Morita, T.; Kimura, Y.; Tsujii, M.; Hayashi, N.; Sobue, K. (2007): Caldesmon suppresses cancer cell invasion by regulating podosome/invadopodium formation. In FEBS Lett 581 (20), pp. 3777-3782.

Yund, Erin E.; Hill, Joseph A.; Keller, Rebecca S. (2009): Hic-5 is required for fetal gene expression and cytoskeletal organization of neonatal cardiac myocytes. In Journal of molecular and cellular cardiology 47 (4), pp. 520-527.

Zaidel-Bar, Ronen; Itzkovitz, Shalev; Ma'ayan, Avi; Iyengar, Ravi; Geiger, Benjamin (2007): Functional atlas of the integrin adhesome. In Nat Cell Biol 9 (8), pp. 858-867.

Zhu, Liang; Zhu, B. I.N.; Yang, Luoyan; Zhao, Xiaokun; Jiang, Honhyi; Ma, Fang (2014): RelB regulates $\mathrm{Bcl}-\mathrm{xl}$ expression and the irradiation-induced apoptosis of murine prostate cancer cells. In Biomed Rep 2 (3), pp. 354-358.

Zutter, M. M.; Ryan, E. E.; Painter, A. D. (1997): Binding of phosphorylated Sp1 protein to tandem Sp1 binding sites regulates alpha2 integrin gene core promoter activity. In Blood 90 (2), pp. 678-689. 


\section{Acknowledgements}

I want to express my deep gratitude to Prof. Dr. Peter Burfeind for being my referee and Supervisor, for the friendly relationship, helpful suggestions, his sympathetic ear and for the fruitful discussions during the whole time.

I would like to thank Prof. Dr. Dieter Kube and Prof. Dr. Holger Bastians for being my thesis committee members.

I am indebted to Dr. Silke Kaulfuß for giving me the opportunity to work on this interesting topic. I also want to thank her for her guidance and encouragement, her helpful suggestions and ideas and for proofreading my manuscripts.

I would like to extend my appreciation to Prof. Dr. mult. Thomas Meyer for his assistance during the EMSA experiments and for the discussions about my project.

My particular gratitude I want to express to my lab mate Lisa, who was doing a great job especially during the important phase of the project. Moreover, I would like to thank her for her sympathetic ear and joyful personality that made lab life even more fun.

Special thanks to my lab colleagues Rovena, Rabea, Julia, Britta, Claudia and Daria for the cheerful and friendly atmosphere inside and outside of the lab and for giving me a hand whenever I needed it. I was very pleased to work in the labs 311, 312 and 307 along with such great personalities. I will miss you!

I would like to thank all people from the institute, for being friendly and helpful.

Let me express my deepest gratitude to my family, for their enormous contribution to this work through their love, encouragement and unconditional support in every imaginable situation. Therefore, I dedicate this thesis to them. 